\title{
Nonalcoholic fatty liver disease in relation to cardiovascular disease
}

Citation for published version (APA):

Simons, N. (2021). Nonalcoholic fatty liver disease in relation to cardiovascular disease: Is all fat equal? [Doctoral Thesis, Maastricht University]. Maastricht University. https://doi.org/10.26481/dis.20210226ns

Document status and date:

Published: 01/01/2021

DOI:

10.26481/dis.20210226ns

Document Version:

Publisher's PDF, also known as Version of record

\section{Please check the document version of this publication:}

- A submitted manuscript is the version of the article upon submission and before peer-review. There can be important differences between the submitted version and the official published version of record.

People interested in the research are advised to contact the author for the final version of the publication, or visit the DOI to the publisher's website.

- The final author version and the galley proof are versions of the publication after peer review.

- The final published version features the final layout of the paper including the volume, issue and page numbers.

Link to publication

\footnotetext{
General rights rights.

- You may freely distribute the URL identifying the publication in the public portal. please follow below link for the End User Agreement:

www.umlib.nl/taverne-license

Take down policy

If you believe that this document breaches copyright please contact us at:

repository@maastrichtuniversity.nl

providing details and we will investigate your claim.
}

Copyright and moral rights for the publications made accessible in the public portal are retained by the authors and/or other copyright owners and it is a condition of accessing publications that users recognise and abide by the legal requirements associated with these

- Users may download and print one copy of any publication from the public portal for the purpose of private study or research.

- You may not further distribute the material or use it for any profit-making activity or commercial gain

If the publication is distributed under the terms of Article $25 \mathrm{fa}$ of the Dutch Copyright Act, indicated by the "Taverne" license above, 


\section{Nonalcoholic Fatty Liver Disease in relation to Cardiovascular Disease}

Is all fat equal? 
Copyright $\odot$ Nynke Simons 2021

All rights reserved. No part of this thesis may be reproduced or distributed in any form or by any means, without the prior written permission of the author or the publisher.

Cover: Jean L.J.M. Scheijen

Layout: Tiny Wouters

Printing: Proefschriftenprinten.nl

ISBN: 978-90-831117-5-9

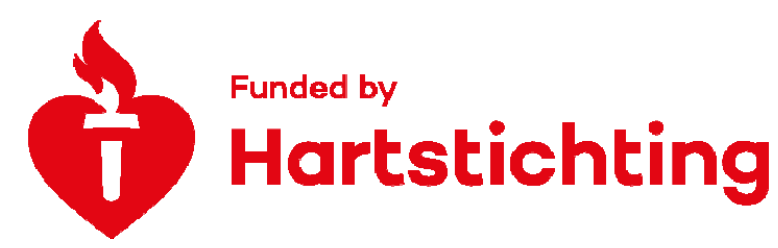

The research described in this thesis was supported by a grant of the Dutch Heart Foundation (2015T042) and financial support by the Dutch Heart Foundation for the publication of this thesis is gratefully acknowledged. 


\title{
Nonalcoholic Fatty Liver Disease in relation to Cardiovascular Disease
}

\author{
Is all fat equal?
}

\author{
PROEFSCHRIFT \\ ter verkrijging van \\ de graad van doctor aan de Universiteit Maastricht, \\ op gezag van de Rector Magnificus, Prof. dr. Rianne M. Letschert, \\ volgens het besluit van het College van Decanen, \\ in het openbaar te verdedigen \\ op vrijdag 26 februari 2021 om 14.00 uur
}

door

Nynke Simons 


\section{Promotores}

Prof. dr. M.C.G.J. Brouwers

Prof. dr. C.D.A. Stehouwer

Prof. dr. N.C. Schaper

\section{Beoordelingscommissie}

Prof. dr. A.A. Kroon (voorzitter)

Dr. A.G. Holleboom (Amsterdam UMC)

Prof. dr. J. Plat

Prof. dr. ir. Y.T. van der Schouw (UMC Utrecht)

Prof. dr. R. Shiri-Sverdlov 


\section{Table of contents}

$\begin{array}{lll}\text { Chapter } 1 \text { General introduction } & 7\end{array}$

Part I Genetic studies to elucidate the relationship between nonalcoholic fatty liver disease and cardiovascular disease

Chapter 2 Interaction between a genetic variant in GCKR and environmental factors

Chapter 3 Effects of genetic variants in GCKR on coronary artery disease, glomerular filtration rate and chronic kidney disease

Chapter 4 Effects of genetic variants in TM6SF2, PNPLA3 and MBOAT7 on coronary artery disease

Chapter 5 Effects of NAFLD susceptibility genes on coronary artery disease in relation to plasma lipids

Part II The relationship between nonalcoholic fatty liver disease and biomarkers of endothelial function

Chapter 6 Soluble E-selectin as a biomarker of hepatic endothelial function

Part III Modulation of fructose metabolism to study the relationship between intrahepatic lipid content and cardiovascular disease

Chapter 7 Intrahepatic lipid content in hereditary fructose intolerance

Chapter 8 Kidney and vascular function in hereditary fructose intolerance

Chapter 9 Intrahepatic lipid content after a six-week fructoserestricted diet

Chapter 10 Summary and general discussion

Chapter 11 Nederlandse samenvatting

Valorisation addendum

Dankwoord

Scientific output 

-

$\checkmark$

-

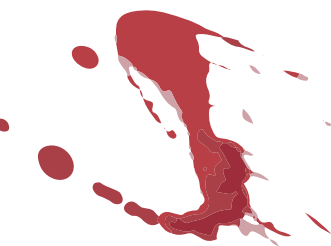

-

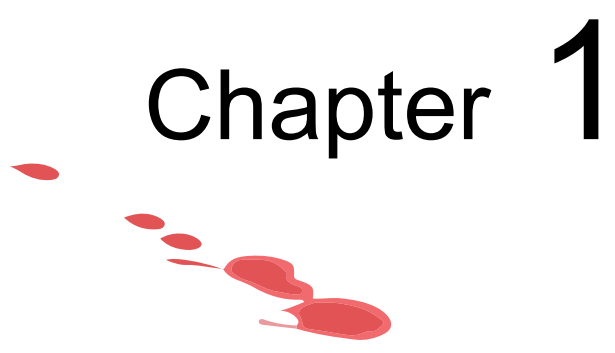

General introduction
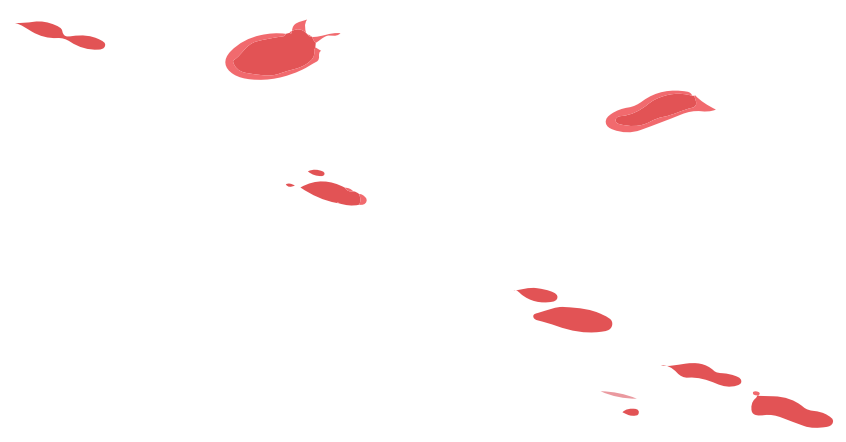
Chapter 1 


\section{Cardiovascular disease and its relationship with nonalcoholic fatty liver disease}

Cardiovascular disease (CVD) is one of the most common causes of death in Europe. ${ }^{1}$ European economic and health care costs related to CVD have reached alarming high rates, with an estimation of more than $€ 200$ billion per year. ${ }^{2}$ Atherosclerosis is the main process underlying CVD; it is caused by an interplay between disturbed blood flow, retention of lipoproteins in the tunica intima (the inner layer of an artery), endothelial dysfunction and inflammation, which in time can result in local thrombosis and ischemia. ${ }^{3}$ The main reversible risk factors for CVD are high blood pressure and LDL cholesterol levels, presence of diabetes mellitus and obesity, smoking and alcohol use, and low fruit/vegetable intake and physical activity. ${ }^{4,5}$ In the last decade, several studies have identified another risk factor for CVD, i.e., nonalcoholic fatty liver disease (NAFLD). ${ }^{6-8}$ However, the exact mechanism underlying the relationship between NAFLD and CVD remains to be elucidated.

\section{Definition, epidemiology and health burden of nonalcoholic fatty liver disease}

NAFLD consists of a histological spectrum comprising hepatic steatosis (affecting $>5 \%$ of hepatocytes), steatohepatitis (the presence of steatosis and lobular inflammation and/or ballooning, hereinafter referred to as nonalcoholic steatohepatitis (NASH)) and peri-sinusoidal fibrosis. ${ }^{9}$ In a number of individuals, NAFLD progresses to advanced fibrosis, cirrhosis and hepatocellular carcinoma. ${ }^{10-12}$ The diagnosis is based on 1) the presence of hepatic steatosis and 2) the absence of excessive alcohol intake or other secondary causes of hepatic steatosis (e.g., steatogenic medication or monogenic hereditary disorders). ${ }^{13}$

The gold standard for the diagnosis of NAFLD is liver histology. ${ }^{9,13}$ This method, however, has several limitations, including invasiveness, risk of (lifethreatening) complications, poor accessibility and sampling variability. ${ }^{14}$ Nowadays, there are several other methods to identify NAFLD, including plasma biomarkers, transient elastography and imaging. ${ }^{15,16}$ Unfortunately, most of these methods are limited by a low sensitivity to detect mild steatosis (e.g., serum aminotransferases and ultrasound) or radiation exposure (e.g., 
computed tomography). ${ }^{13,17,18}$ Proton magnetic resonance spectroscopy $\left({ }^{1} \mathrm{H}-\mathrm{MRS}\right)$ is considered the gold standard for the noninvasive assessment of intrahepatic lipid (IHL) content. ${ }^{19,20}{ }^{1} \mathrm{H}-\mathrm{MRS}$ evaluates proton signals as a function of their resonance frequency (visible as MR spectra), which vary between water and lipid due to different chemical components, thereby making it possible to evaluate the chemical composition of hepatic tissue..$^{21,22}$

The worldwide prevalence of NAFLD is approximately one in every 4 adults and one in every 13 children. ${ }^{23,24}$ The prevalence is gradually increasing (from $15 \%$ in 2005 to $25 \%$ in 2010) and NAFLD is expected to become the most important cause of liver-related death (with a total of 2.14 million in 2017; representing an $11.4 \%$ increase since 2012$)^{25}$ and the principal cause of liver transplantation. ${ }^{26}$ The prevalence rises with age and is more common in males than in females and in Hispanic individuals than Caucasians or African Americans. ${ }^{27,28}$ In addition, NAFLD is strongly associated with other noncommunicable diseases, such as type 2 diabetes, chronic kidney disease and cancer. $^{23,29}$ In fact, the primary cause of death in individuals with NAFLD is CVD. ${ }^{30}$ Hitherto, two pharmacological interventions have been shown to affect NAFLD as well as CVD. Pioglitazone and liraglutide both effectively resolve $\mathrm{NASH}$ (number needed to treat $\sim 3$ within 18 months and 48 weeks, respectively) ${ }^{31,32}$ and prevent major adverse cardiovascular events in type 2 diabetes. $^{33,34}$ Nevertheless, the development and implementation of new therapeutic targets have been a challenge primarily due to the difficulty to noninvasively diagnose NAFLD (in particular the more advanced stages (i.e., steatohepatitis and fibrosis)).

\section{Pathophysiology of nonalcoholic fatty liver disease}

\subsection{Pathways underlying intrahepatic lipid accumulation}

The liver plays a central role in lipid metabolism, since it is involved in the synthesis, excretion and distribution of fatty acids as well as their utilization as an energy substrate. ${ }^{35}$ These processes are tightly regulated by hormones, nuclear receptors and transcription factors. ${ }^{36}$ Disturbance in either the input or output of lipids or regulators of lipid metabolism could, therefore, result in retention of lipids in the liver and subsequently the development of NAFLD. ${ }^{37}$

There are four pathways that can result in IHL accumulation: 1) increased flux of free fatty acids derived from the adipose tissue or diet, 2) increased hepatic de novo lipogenesis from simple sugars, such as glucose and fructose, 3) 
decreased hepatic oxidation of fatty acids or 4) decreased secretion of triglyceride-rich very low-density lipoprotein (VLDL) particles. ${ }^{38,39}$

The impact of each pathway on the accumulation of intrahepatic lipids varies and depends on many factors, such as physical condition, nutritional status and genetic background. ${ }^{40,41}$ However, stable isotope studies have shown that, in NAFLD, triglyceride-rich VLDL secretion ${ }^{42-44}$ and fatty acid oxidation ${ }^{45-47}$ are increased, suggesting that increased lipid formation rather than decreased output underlies IHL accumulation in NAFLD. Indeed, the contribution of de novo lipogenesis to IHL formation is strongly upregulated in individuals with NAFLD (approximately $20-40 \%$ compared to $10 \%$ in healthy individuals). ${ }^{48,49}$

A proportion of individuals with NAFLD (10-30\%) evolve to the second, inflammatory stage of NAFLD, i.e., $\mathrm{NASH}^{50}$ In addition to $\mathrm{IHL}$ deposition, $\mathrm{NASH}$ is characterized by the infiltration of inflammatory cells and subsequent cell injury (i.e., hepatocyte ballooning). The toxic effect of lipids on hepatocytes (lipotoxicity) is the driving force behind NASH development. Although the factors responsible for the progression from steatosis to steatohepatitis in NAFLD have not been fully elucidated, experimental studies have shown that the amount and type of lipids that accumulate in the hepatocyte determine lipotoxicity and, hence, progression to NASH. Particularly (an excess of) fatty acids seem to be hepatotoxic ${ }^{51,52}$, amongst others via the production of reactive oxygen species accompanying mitochondrial and peroxisomal fatty acid oxidation. $^{53}$

\subsection{Risk factors}

The phenotypic manifestation and severity of NAFLD is the result of multiple gene-environment interactions. ${ }^{54}$

\subsubsection{Genetic variants}

Genetic predisposition for NAFLD has been widely studied and several NAFLD susceptibility genes have been identified. Up to now, four genetic variants have consistently been associated with all stages of NAFLD. ${ }^{55}$

The first genetic risk factor identified was rs738409 $(C>G){ }^{56,57}$ This single nucleotide polymorphism (SNP) encodes an isoleucine-to-methionine variation at amino acid position 148 of the patatin-like phospholipase domain-containing protein 3 (PNPLA3) protein. The exact mechanism by which this genetic variant predisposes to NAFLD is not entirely clear, but it appears to affect lipid droplet remodeling, leading to decreased triglyceride-rich VLDL production and 
secretion. ${ }^{58-60}$ Indeed, individuals carrying this variant are characterized by lower plasma triglyceride levels. ${ }^{61}$

Second, the gene encoding transmembrane 6 superfamily member 2 (TM6SF2) is a membrane protein that functions as a lipid transporter. ${ }^{62}$ The rs58542926 C>T glutamate-to-lysine variation at amino acid position 167 affects this process by decreased secretion of triglyceride-rich VLDL particles, leading to increased lipid accumulation and hepatocellular lipid droplet size. ${ }^{62,63}$

The third genetic variant associated with NAFLD is the membrane-bound $\mathrm{O}$ acyltransferase domain-containing 7 (MBOAT7) rs641738 C>T variant. ${ }^{64}$ The enzyme encoded by this genetic variant is involved in the remodeling of phospholipids, known as the Lands' cycle (specifically it incorporates arachidonic acids and other unsaturated fatty acids). ${ }^{65}$ The genetic variant leads to decreased MBOAT7 activity and hence altered phospholipid remodeling in hepatocellular lipid droplets, thereby predisposes to NAFLD. ${ }^{64,65}$

Last, the glucokinase regulatory protein gene (GCKR) encodes a liver specific protein that binds and inactivates glucokinase in the nucleus of the hepatocyte. $^{66}$ In the postprandial phase, this complex is dissociated and glucokinase is able to migrate to the cytosolic space for the conversion of glucose to glucose-6-phosphate, i.e., the initiation of glycolysis ${ }^{66}$ and, amongst others, subsequent lipogenesis (Figure 1.1). The rs1260326 (C>T) proline-toleucine variation at amino acid position 446 encodes a protein that binds glucokinase less effectively, leading to increased glucokinase activity and, hence, increased hepatic glucose uptake and glycolysis. ${ }^{67}$ Experimental studies have shown that $r s 1260326$ is a functional variant ${ }^{67}$ and the genetic variant has been associated with lower plasma glucose and insulin levels, increased de novo lipogenesis, NAFLD and higher plasma triglyceride levels. ${ }^{68-72}$

\subsubsection{Lifestyle and nutrition}

An unhealthy lifestyle (mainly overnutrition and lack of physical activity) is one of the major factors contributing to the current NAFLD epidemic. Increased caloric intake induces (visceral) adipose tissue expansion, de novo lipogenesis, insulin resistance and gut dysbiosis, which all (in)directly contribute to the development of NALFD. ${ }^{17}$ Besides an increased caloric intake per se, there is discussion about the contribution of individual macronutrients to $\mathrm{IHL}$ accumulation. Despite a decrease over the last decades, the excessive intake 
of saturated fat and sugar - both well-known cardiovascular risk factors ${ }^{73,74}$ has remained higher than recommended. ${ }^{75,76}$ Saturated fat is known to increase IHL content via stimulation of adipose tissue lipolysis, whereas simple sugars induce IHL accumulation via increased de novo lipogenesis. ${ }^{77}$

Specifically fructose has been suggested to play a key role in the development of NAFLD, since it is primarily metabolized in the liver. ${ }^{78}$ In addition, opposed to the tight regulation of glucose, fructose is almost entirely taken up by the liver without any regulation and converted to glucose, lactate, glycogen or lipids. ${ }^{79}$ The precise mechanism by which fructose causes IHL accumulation, however, is not entirely clear. Fructose can contribute to the development of NAFLD either as a substrate for de novo lipogenesis (via conversion of fructose to glyceraldehyde-3-phosphate (i.e., fructolysis; Figure 1.1)) ${ }^{79}$ or as a signal molecule that induces transcription factors that enable the expression of genes involved in de novo lipogenesis (such as sterol regulatory element binding protein 1 (SREBP1), carbohydrate response element binding protein $($ ChREBP) and liver $X$ receptor $(L X R)) .{ }^{80,81}$ Further, fructose-1-phosphate, an intermediate of fructolysis, facilitates hepatic glucose uptake by dissociation of glucokinase from glucokinase regulatory protein $(\mathrm{GKRP})^{66}$, which might end up being stored as lipids via de novo lipogenesis (Figure 1.1).

Laboratory studies have confirmed that fructose is converted into glycerol and fatty acids ${ }^{82}$ and that it increases the expression of lipogenic transcription factors. ${ }^{83}$ Moreover, interference with hepatic fructose metabolism, for example by inhibition of ketohexokinase (the enzyme responsible for the conversion of fructose to fructose-1-phosphate; Figure 1.1 $)^{84}$, prevents IHL accumulation in mice. ${ }^{83,85}$ Clinical studies in humans investigating the relationship between fructose and NAFLD are, however, conflicting. A meta-analysis did not demonstrate any change in IHL content after dietary interventions with added fructose when compared to added glucose. ${ }^{86}$ Studies that have demonstrated a change in IHL content often used a fructose overload in combination with a hypercaloric diet. ${ }^{87-90}$ In contrast, a nine-day isocaloric fructose-restricted diet successfully reduced IHL content of obese children with a high habitual sugar intake, which was mainly attributed to a decrease in de novo lipogenesis. ${ }^{91}$ This pre-post study, however, was greatly limited by the lack of a control group. Thus, the effect of fructose one IHL accumulation per se, remains to be disputed. 


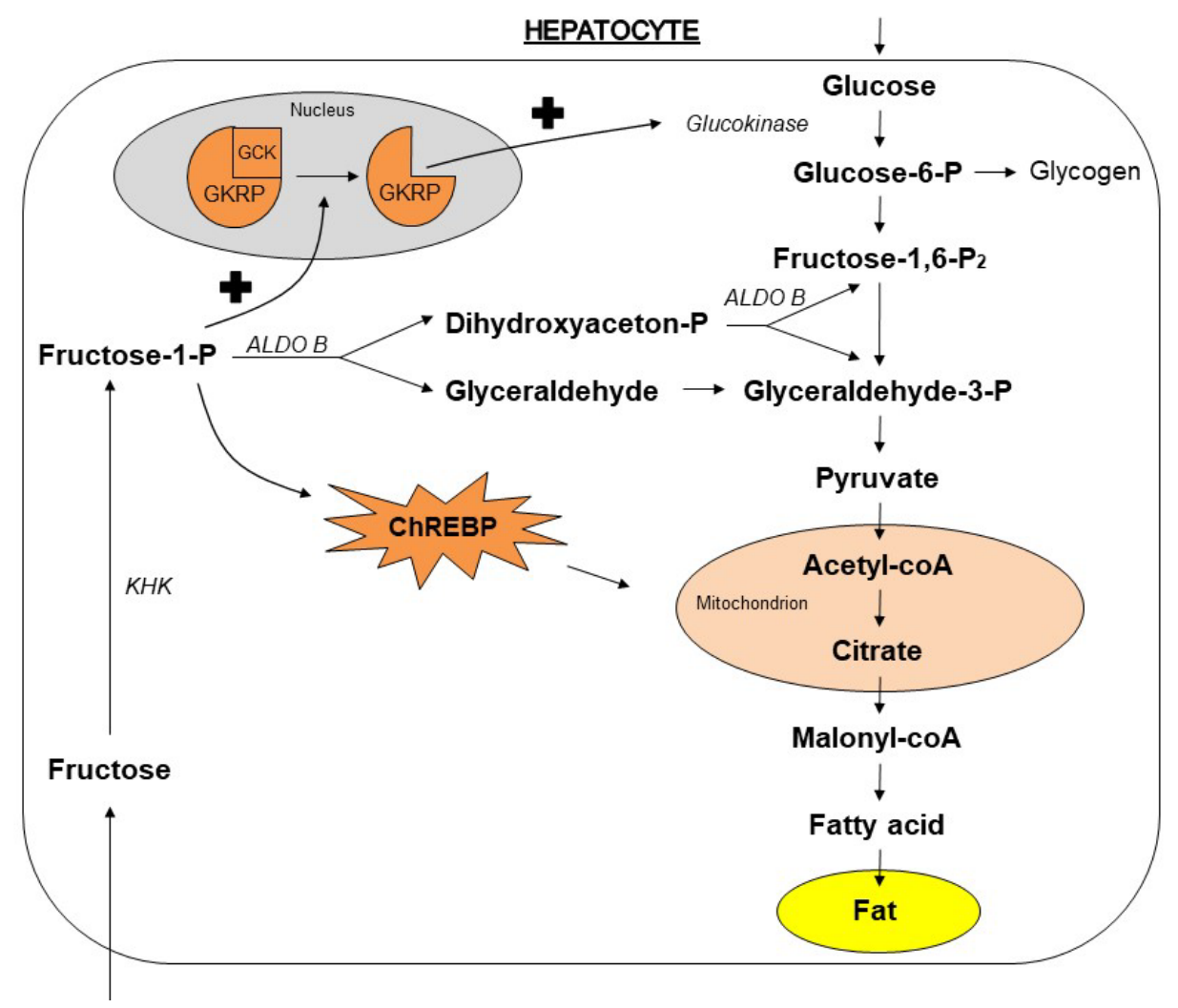

Figure 1.1 Role of glucokinase regulatory protein and fructose in intrahepatic lipid accumulation. Simplified schematic overview of the role of glucokinase regulatory protein and fructose in hepatic glycolysis and de novo lipogenesis. Figure adapted from Brouwers, Trends Mol Med (2015). Abbreviations: glucose-6-phosphate (glucose-6-P), fructose-1,6-diphosphate (fructose-1,6- $\mathrm{P}_{2}$ ), glyceraldehyde-3-phosphate (glyceraldehyde-3-P), acetyl coenzyme A (acetyl-coA), malonyl coenzyme A (malonyl-coA), fructose-1-phosphate (fructose-1-P), aldolase B (ALDO B) dihydroxyaceton-phosphate (dihydroxyaceton- $P$ ), glucokinase regulatory protein (GKRP), glucokinase (GCK), carbohydrate-responsive element-binding protein (ChREBP), ketohexokinase (KHK).

\subsubsection{Insulin resistance}

Insulin resistance is a crucial factor in the pathophysiology of NAFLD, and vice versa. $^{92}$ Type 2 diabetes is present in $23 \%$ of individuals with NAFLD ${ }^{23}$ and up to $70 \%$ of individuals with type 2 diabetes have NAFLD. ${ }^{93,94}$

Insulin normally suppresses adipose tissue lipolysis and hepatic glucose and triglyceride-rich VLDL production, and stimulates glycogen and lipid synthesis. 
In the presence of insulin resistance, however, intrahepatic lipids accumulate due to an impaired suppression of lipolysis in the adipose tissue (resulting in an increased flux of free fatty acids towards the liver). ${ }^{95}$ In addition, hyperinsulinemia contributes to $\mathrm{IHL}$ accumulation by stimulating de novo lipogenesis via activation of SREBP1 and LXR, which in turn enable hepatic expression of lipogenic genes (e.g., acetyl-coenzyme A carboxylase and fatty acid synthase). ${ }^{80}$ Elevated plasma glucose by itself also contributes to IHL accumulation by serving as a substrate for de novo lipogenesis ${ }^{49}$ and inducing transcription factors involved in de novo lipogenesis (i.e., ChREBP). ${ }^{96}$ In turn, stable isotope studies have shown that $\mathrm{IHL}$ is associated with an impaired suppression of endogenous glucose production. ${ }^{97,98}$ Moreover, several longitudinal studies have identified NAFLD as a predictor of incident type 2 diabetes. ${ }^{94,99,100}$

Interestingly, not all individuals who accumulate intrahepatic lipids display the same increased metabolic risk. Although the majority of individuals with NAFLD are characterized by insulin resistance ${ }^{41}$, individuals with familial hypobetalipoproteinemia have an increased $\mathrm{IHL}$ content but normal insulin sensitivity. ${ }^{101}$ Whether this difference in metabolic risk is also true for cardiovascular risk is not known.

\section{Mechanisms connecting nonalcoholic fatty liver disease with cardiovascular disease}

Although NAFLD has been identified as an independent risk factor for the development of CVD in several high-quality meta-analyses ${ }^{6-8}$, it remains unclear whether NAFLD is an active contributor to cardiovascular risk or just an innocent bystander. Several mediators have been suggested for the association between NAFLD and CVD (Figure 1.2). ${ }^{102,103}$ 


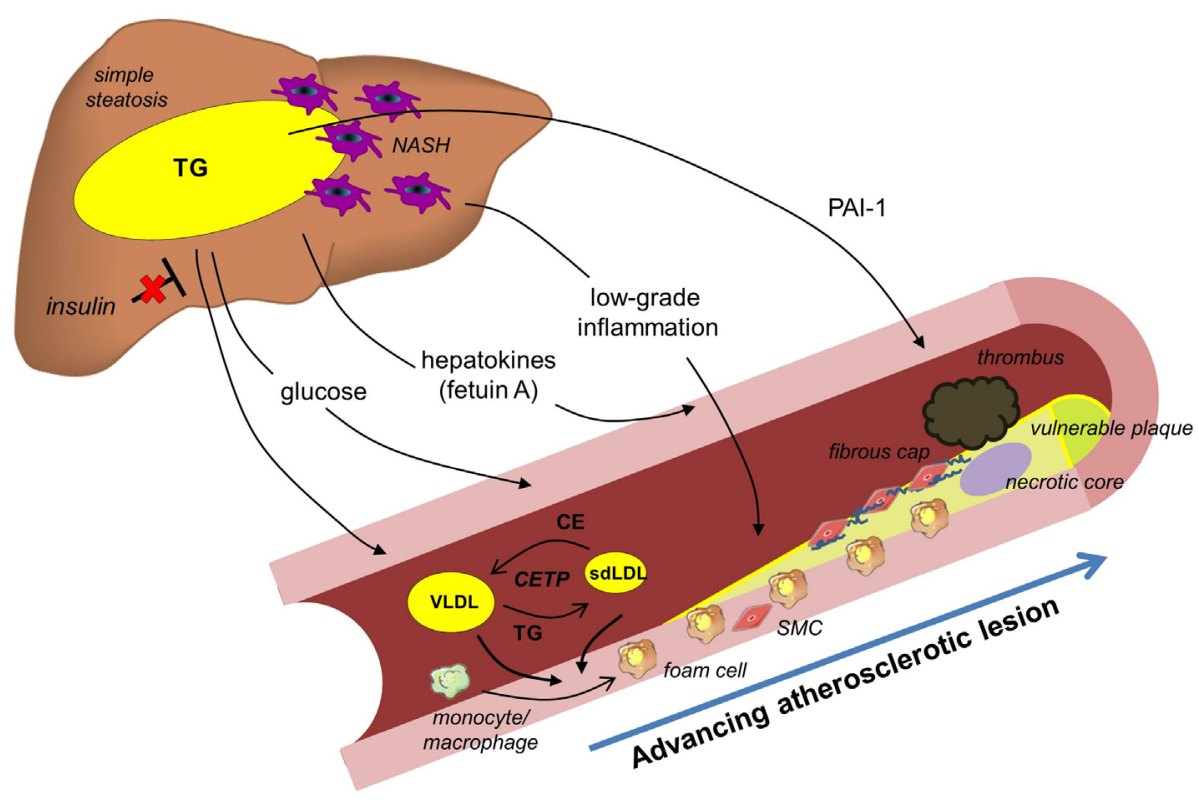

Figure 1.2 Possible underlying mechanisms linking nonalcoholic fatty liver disease to cardiovascular disease Overview of the possible underlying mechanisms linking NAFLD to (the pathogenesis of) CVD. Figure adapted from Brouwers, Diabetologia (2020). Abbreviations: triglycerides (TG), steatohepatitis (NASH), plasminogen activator inhibitor (PAl-1), very-low-density lipoprotein (VLDL), small dense low-density lipoprotein (sdLDL), cholesteryl ester (CE), cholesteryl ester transfer protein (CETP), smooth muscle cell (SMC).

\subsection{Dyslipidemia}

As hitherto mentioned, the liver is a central organ in lipid metabolism. NAFLD is associated with a typical atherogenic dyslipidemia, characterized by high plasma triglycerides levels, high numbers of triglyceride-rich VLDL and smalldense low-density lipoprotein (sdLDL) particles, and low plasma high-density lipoprotein (HDL) cholesterol levels. ${ }^{104-106} \mathrm{IHL}$ content is associated with insulinresistant overproduction of triglyceride-rich VLDL particles ${ }^{107}$, especially in the postprandial phase. ${ }^{108}$ This overproduction is mainly driven by de novo lipogenesis $^{48}$ (which, under normal conditions, is only a minor contributor to plasma triglyceride levels). The presence of triglyceride-rich VLDL particles drives the cholesteryl ester transfer protein (CETP) mediated exchange of cholesteryl esters and triglycerides between VLDL and HDL and LDL particles ${ }^{109}$, which leads to the generation of cholesterol-depleted HDL and LDL particles (i.e., sdLDL) ${ }^{110}$ (Figure 1.2). sdLDL cholesterol is a well-known 
contributor to CVD. In addition, plasma triglycerides have been causally linked to coronary artery disease $(C A D)$ in a Mendelian randomization study. ${ }^{111}$

\subsection{Low-grade inflammation}

Low-grade inflammation is a common feature of both NASH and atherosclerosis. ${ }^{112,113}$ The necroinflammatory environment that characterizes $\mathrm{NASH}$ may contribute to the systemic inflammatory state that underlies CVD via secretion of proinflammatory cytokines and proteins (Figure 1.2). Indeed, the transcription of genes that augment systemic low-grade inflammation is upregulated in individuals with $\mathrm{NASH} .{ }^{14,115}$ Moreover, systemic inflammation biomarkers (e.g., interleukin-6, C-reactive protein, tumor necrosis factor alpha, soluble intracellular adhesion molecule 1) are elevated in NAFLD. ${ }^{103,116-119}$ Finally, a large randomized controlled trial has demonstrated that a monoclonal antibody targeting interleukin-1 (which is also involved in the pathogenesis of $\mathrm{NASH}^{120}$ ) successfully reduces cardiovascular events. ${ }^{121}$

\subsection{Hypercoagulation and impaired fibrinolysis}

As the liver plays an important role in coagulation, an abnormal secretion of coagulation or fibrinolytic factors can contribute to a prothrombotic state, as often seen in individuals with cardiovascular events. ${ }^{122,123}$ Indeed, a higher hepatic expression and plasma levels of plasminogen activator inhibitor type 1 (PAl-1; an inhibitor of fibrinolysis) and various coagulation factors have been found in individuals with NAFLD. ${ }^{124-127}$ Additionally, diacylglycerol - an intermediate of de novo lipogenesis - indirectly affects fibrinolysis via increased expression of PAI-1. ${ }^{128}$ Mendelian randomization studies have inferred a causal effect of PAI-1 on CAD. ${ }^{129}$

\subsection{Hepatokines}

Proteins derived from the liver that affect metabolic processes via autocrine, paracrine and endocrine signaling (so-called hepatokines) ${ }^{130}$ have also been proposed as potential mediators of cardiometabolic complications. ${ }^{131}$ For example, the expression of fetuin $A$ is elevated in steatotic mouse livers ${ }^{132}$ and plasma fetuin A levels are higher in individuals with NAFLD. ${ }^{133-135}$ In turn, Fetuin $A$ levels are associated with markers of atherosclerosis ${ }^{136,137}$ as well as cardiovascular events. ${ }^{138,139}$ Activation of inflammatory signaling is thought to be the underlying mechanism by which Fetuin A links NAFLD to CVD. ${ }^{140,141}$ 


\subsection{Insulin resistance, hyperinsulinemia and hyperglycemia}

As described in paragraph 3.2.3, the liver plays an important role in glucose homeostasis and NAFLD is associated with insulin resistance and type 2 diabetes. ${ }^{94,142}$ Hepatic de novo lipogenesis has been associated with advanced glycation end products, carbohydrate (sugar) intake, hyperglycemia and hyperinsulinemia. Type 2 diabetes is a well-known risk factor for CVD and plasma glucose levels have recently been causally linked to CAD in a Mendelian randomization study. ${ }^{143}$

\section{Aims and outline of this thesis}

Based on previously reported literature, several questions arise concerning the association between NAFLD and CVD. Although NAFLD is a known risk factor for CVD, it is unclear whether NAFLD is an active contributor to CVD or just an innocent bystander. Moreover, not all individuals with NAFLD appear to have the same metabolic risk. Since IHL accumulation can result from different pathways, the specific pathway leading to IHL accumulation - in particular de novo lipogenesis - might determine the risk of comorbidities (as outlined in paragraph 4). The two principal questions of this thesis are therefore:

1. Is NAFLD causally related to CVD?

2. Does the relationship between NAFLD and CVD depend on the pathway that leads to IHL accumulation?

Based on the current scientific evidence, I hypothesize that NAFLD is an active contributor to cardiovascular risk and particularly de novo lipogenesis is disadvantageous for cardiovascular health.

To objectively assess the presence of a causal relationship between NAFLD and CVD in humans, randomized controlled trials are mandatory. Unfortunately, the two pharmacological interventions that have been shown to affect both NAFLD and CVD (i.e., pioglitazone ${ }^{31,33}$ and liraglutide ${ }^{32,34}$ ), do not specifically target NAFLD. Hence, a causal effect cannot be concluded. Furthermore, stable isotope studies in large study populations are required to study the different pathways leading to NAFLD and their relationship with CVD. For example, de novo lipogenesis can be measured with the rate by which palmitate is incorporated in plasma triglycerides (derived from the liver) using isotope tracers. ${ }^{144}$ Unfortunately, the implementation of these expensive stable isotope studies in large number of participants is practically impossible. 
These practical limitations can be overcome by studying the cardiometabolic effects of different NAFLD susceptibility genes that predispose to IHL accumulation via different metabolic pathways (i.e., increased de novo lipogenesis [GCKR], decreased production and/or secretion of triglyceride-rich VLDL particles [PNPLA3 and TM6SF2] and impaired mobilization (and thus usage/utilization) of triglycerides [MBOAT7]) on a population level. Since an individual is 'randomized' at conception to receive either a protective or predisposing NAFLD risk allele, causal inferences can be made in observational studies using these genetic variants. This Mendelian randomization approach has been widely adopted. ${ }^{145,146}$ The variant in GCKR (rs1260326) is particularly suitable to study the cardiometabolic effects of the de novo lipogenesis pathway in NAFLD, as it has a high allele frequency $(\sim 34 \%)^{147}$, is liver specific ${ }^{66}$ and has been associated with NAFLD ${ }^{71}$ as well as de novo lipogenesis. ${ }^{72}$

In chapter two of this thesis, we examined whether the association between rs1260326 (GCKR) and plasma lipid levels was different between individuals with a normal glucose metabolism, individuals with an impaired glucose metabolism or individuals with type 2 diabetes. For this we used the combined CODAM and Hoorn studies, which are two prospective cohorts designed to study determinants and (cardiovascular) complications of type 2 diabetes.

In chapter three, we performed a systematic literature review and metaanalysis to study the relationship between multiple variants in GCKR and CAD, estimated glomerular filtration rate and chronic kidney disease.

In chapter four, we assessed the association between three genetic variants that predispose to NAFLD via other pathways than GCKR (i.e., PNPLA3, TM6SF2 and MBOAT7) and CAD in approximately 180,000 individuals from the Coronary Artery Disease Genome-Wide Replication and Meta-Analysis plus the Coronary Artery Disease Genetics (CARDloGRAMplusC4D) Consortium dataset.

In chapter five, we studied the association between all currently reported NAFLD susceptibility genes and CAD in relation to plasma lipid levels, using two large datasets.

In chapter six, we assessed the relationship between rs1260326 (GCKR) and rs738409 (PNPLA3) and plasma biomarkers of endothelial (dys)function (i.e., soluble endothelium selectin and soluble vascular cell adhesion molecule 1), one of the first stages of the atherosclerotic process ${ }^{148}$, in the combined 
CODAM and Hoorn studies. As there is an indication that E-selectin is also involved in the pathogenesis of alcoholic fatty liver disease ${ }^{149}$ (which is histologically similar to NAFLD), we also assessed the possible origin of E-selectin in livers obtained from an experimental animal study and individuals undergoing bariatric surgery.

In addition to genetic variants, the cardiometabolic effects of an enhanced dissociation of glucokinase from glucokinase regulatory protein (and, consequently, increased de novo lipogenesis) can theoretically also be studied with fructose. As previously described, fructose-1-phosphate, an intermediate of fructose metabolism, disrupts the binding between glucokinase and glucokinase regulatory protein. As a consequence, glucokinase facilitates the conversion of glucose to glucose-6-phosphate, thereby increasing hepatic glucose uptake and providing a substrate for de novo lipogenesis (Figure 1.1).

In chapter seven, we assessed the IHL content in patients with hereditary fructose intolerance $(\mathrm{HFI})$ in comparison to healthy control subjects matched for age, sex and body mass index (BMI). HFI is a rare inborn error that is characterized by accumulation of fructose-1-phosphate as a consequence of a deficiency in aldolase $B^{150}$ (Figure 1.1). In addition to the $\mathrm{IHL}$ content and glucose metabolism, we also compared the kidney and vascular function in HFI patients with age-, sex- and BMI-matched control subjects (chapter eight).

In chapter nine, we investigated the effects of fructose restriction on IHL content, glucose tolerance and plasma lipids in 44 adult overweight individuals in a double-blind randomized controlled trial.

Last, in chapter ten, I discuss the key findings of the thesis as well as the methodological considerations of the studies reported. In addition, I consider the clinical applications of the primary results and propose suggestions for future research. 


\section{Literature}

1. Timmis A, Townsend N, Gale CP, et al. European Society of Cardiology: Cardiovascular Disease Statistics 2019. Eur Heart J 2020;41(1):12-85.

2. Wilkins E, Wilson L, Wickramasinghe $K$, Bhatnagar $P$, Leal J, Luengo-Fernandez R, Burns R, Rayner M, Townsend N. European Cardiovascular Disease Statistics 2017. Brussels: European Heart Network; 2017.

3. Tabas I, Garcia-Cardena G, Owens GK. Recent insights into the cellular biology of atherosclerosis. J Cell Biol 2015;209:13-22.

4. Hennekens $\mathrm{CH}$. Increasing burden of cardiovascular disease: current knowledge and future directions for research on risk factors. Circulation 1998;97:1095-102.

5. Yusuf $\mathrm{S}$, Hawken $\mathrm{S}$, Ounpuu $\mathrm{S}$, et al. Effect of potentially modifiable risk factors associated with myocardial infarction in 52 countries (the INTERHEART study): case-control study. Lancet 2004;364:937-52.

6. Targher G, Byrne CD, Lonardo A, Zoppini G, Barbui C. Non-alcoholic fatty liver disease and risk of incident cardiovascular disease: A meta-analysis. J Hepatol 2016;65:589-600.

7. Mahfood Haddad T, Hamdeh S, Kanmanthareddy A, Alla VM. Nonalcoholic fatty liver disease and the risk of clinical cardiovascular events: A systematic review and meta-analysis. Diabetes Metab Syndr 2017;11 Suppl 1:S209-s16.

8. Wu S, Wu F, Ding Y, Hou J, Bi J, Zhang Z. Association of non-alcoholic fatty liver disease with major adverse cardiovascular events: A systematic review and meta-analysis. Sci Rep 2016;6:33386.

9. EASL-EASD-EASO Clinical Practice Guidelines for the management of non-alcoholic fatty liver disease. J Hepatol 2016;64:1388-402.

10. Pais R, Charlotte F, Fedchuk L, et al. A systematic review of follow-up biopsies reveals disease progression in patients with non-alcoholic fatty liver. J Hepatol 2013;59:550-6.

11. Caldwell SH, Oelsner DH, lezzoni JC, Hespenheide EE, Battle EH, Driscoll CJ. Cryptogenic cirrhosis: clinical characterization and risk factors for underlying disease. Hepatology 1999; 29:664-9.

12. Ascha MS, Hanouneh IA, Lopez R, Tamimi TA, Feldstein AF, Zein NN. The incidence and risk factors of hepatocellular carcinoma in patients with nonalcoholic steatohepatitis. Hepatology 2010;51:1972-8.

13. Chalasani N, Younossi Z, Lavine JE, et al. The diagnosis and management of nonalcoholic fatty liver disease: Practice guidance from the American Association for the Study of Liver Diseases. Hepatology 2018;67:328-57.

14. Sanai FM, Keeffe EB. Liver biopsy for histological assessment: The case against. Saudi J Gastroenterol 2010;16:124-32.

15. Castera L, Friedrich-Rust M, Loomba R. Noninvasive Assessment of Liver Disease in Patients With Nonalcoholic Fatty Liver Disease. Gastroenterology 2019;156:1264-81.e4.

16. Wong VW, Adams LA, de Ledinghen V, Wong GL, Sookoian S. Noninvasive biomarkers in NAFLD and NASH - current progress and future promise. Nat Rev Gastroenterol Hepatol 2018;15:461-78.

17. Stefan N, Haring HU, Cusi K. Non-alcoholic fatty liver disease: causes, diagnosis, cardiometabolic consequences, and treatment strategies. Lancet Diabetes Endocrinol 2019; 7:313-24.

18. Younossi ZM, Loomba R, Anstee QM, et al. Diagnostic modalities for nonalcoholic fatty liver disease, nonalcoholic steatohepatitis, and associated fibrosis. Hepatology 2018;68:349-60.

19. van Werven JR, Marsman HA, Nederveen AJ, et al. Assessment of hepatic steatosis in patients undergoing liver resection: comparison of US, CT, T1-weighted dual-echo MR imaging, and point-resolved 1H MR spectroscopy. Radiology 2010;256:159-68. 
20. Szczepaniak LS, Nurenberg P, Leonard D, et al. Magnetic resonance spectroscopy to measure hepatic triglyceride content: prevalence of hepatic steatosis in the general population. Am J Physiol Endocrinol Metab 2005;288:E462-8.

21. Schwenzer NF, Springer F, Schraml C, Stefan N, Machann J, Schick F. Non-invasive assessment and quantification of liver steatosis by ultrasound, computed tomography and magnetic resonance. J Hepatol 2009;51:433-45.

22. Reeder SB, Cruite I, Hamilton G, Sirlin CB. Quantitative Assessment of Liver Fat with Magnetic Resonance Imaging and Spectroscopy. J Magnc Reson Imaging 2011;34:729-49.

23. Younossi ZM, Koenig AB, Abdelatif D, Fazel Y, Henry L, Wymer M. Global epidemiology of nonalcoholic fatty liver disease-Meta-analytic assessment of prevalence, incidence, and outcomes. Hepatology 2016;64:73-84.

24. Anderson EL, Howe LD, Jones HE, Higgins JP, Lawlor DA, Fraser A. The Prevalence of NonAlcoholic Fatty Liver Disease in Children and Adolescents: A Systematic Review and MetaAnalysis. PloS One 2015;10:e0140908.

25. Paik JM, Golabi P, Younossi Y, Mishra A, Younossi ZM. Changes in the Global Burden of Chronic Liver Diseases From 2012 to 2017: The Growing Impact of Nonalcoholic Fatty Liver Disease. Hepatology 2020.

26. Charlton MR, Burns JM, Pedersen RA, Watt KD, Heimbach JK, Dierkhising RA. Frequency and outcomes of liver transplantation for nonalcoholic steatohepatitis in the United States. Gastroenterology 2011;141:1249-53.

27. Koehler EM, Schouten JN, Hansen BE, et al. Prevalence and risk factors of non-alcoholic fatty liver disease in the elderly: results from the Rotterdam study. J Hepatol 2012;57: 1305-11.

28. Browning JD, Szczepaniak LS, Dobbins R, et al. Prevalence of hepatic steatosis in an urban population in the United States: impact of ethnicity. Hepatology 2004;40:1387-95.

29. Armstrong MJ, Adams LA, Canbay A, Syn WK. Extrahepatic complications of nonalcoholic fatty liver disease. Hepatology 2014;59:1174-97.

30. Ong JP, Pitts A, Younossi ZM. Increased overall mortality and liver-related mortality in nonalcoholic fatty liver disease. J Hepatol 2008;49:608-12.

31. Cusi K, Orsak B, Bril F, et al. Long-Term Pioglitazone Treatment for Patients With Nonalcoholic Steatohepatitis and Prediabetes or Type 2 Diabetes Mellitus: A Randomized Trial. Ann Intern Med 2016;165:305-15.

32. Armstrong MJ, Gaunt P, Aithal GP, et al. Liraglutide safety and efficacy in patients with nonalcoholic steatohepatitis (LEAN): a multicentre, double-blind, randomised, placebo-controlled phase 2 study. Lancet 2016;387:679-90.

33. Dormandy JA, Charbonnel B, Eckland DJ, et al. Secondary prevention of macrovascular events in patients with type 2 diabetes in the PROactive Study (PROspective pioglitAzone Clinical Trial In macroVascular Events): a randomised controlled trial. Lancet 2005;366: 1279-89.

34. Marso SP, Daniels GH, Brown-Frandsen K, et al. Liraglutide and Cardiovascular Outcomes in Type 2 Diabetes. N Engl J Med 2016;375:311-22.

35. Nguyen P, Leray V, Diez M, et al. Liver lipid metabolism. J Anim Physiol Anim Nutr 2008; 92:272-83.

36. Bechmann LP, Hannivoort RA, Gerken G, Hotamisligil GS, Trauner M, Canbay A. The interaction of hepatic lipid and glucose metabolism in liver diseases. J Hepatol 2012;56: 952-64.

37. Roden M. Mechanisms of Disease: hepatic steatosis in type 2 diabetes--pathogenesis and clinical relevance. Nature clinical practice Endocrinol Metab 2006;2:335-48.

38. Friedman SL, Neuschwander-Tetri BA, Rinella M, Sanyal AJ. Mechanisms of NAFLD development and therapeutic strategies. Nat Med 2018;24:908-22.

39. Ipsen DH, Lykkesfeldt J, Tveden-Nyborg P. Molecular mechanisms of hepatic lipid accumulation in non-alcoholic fatty liver disease. Cell Mol Life Sci 2018;75:3313-27. 
40. Kawano $\mathrm{Y}$, Cohen DE. Mechanisms of hepatic triglyceride accumulation in non-alcoholic fatty liver disease. J Gastroenterol 2013;48:434-41.

41. Donnelly KL, Smith Cl, Schwarzenberg SJ, Jessurun J, Boldt MD, Parks EJ. Sources of fatty acids stored in liver and secreted via lipoproteins in patients with nonalcoholic fatty liver disease. J Clin Invest 2005;115:1343-51.

42. Fabbrini E, Mohammed BS, Magkos F, Korenblat KM, Patterson BW, Klein S. Alterations in adipose tissue and hepatic lipid kinetics in obese men and women with nonalcoholic fatty liver disease. Gastroenterology 2008;134:424-31.

43. Adiels M, Boren J, Caslake MJ, et al. Overproduction of VLDL1 driven by hyperglycemia is a dominant feature of diabetic dyslipidemia. Arterioscler Thromb Vasc Biol 2005;25:1697-703.

44. Fabbrini E, Magkos F, Mohammed BS, et al. Intrahepatic fat, not visceral fat, is linked with metabolic complications of obesity. Proc Natl Acad Sci U S A 2009;106:15430-5.

45. Sanyal AJ, Campbell-Sargent C, Mirshahi F, et al. Nonalcoholic steatohepatitis: association of insulin resistance and mitochondrial abnormalities. Gastroenterology 2001;120:1183-92.

46. Chalasani N, Gorski JC, Asghar MS, et al. Hepatic cytochrome P450 2E1 activity in nondiabetic patients with nonalcoholic steatohepatitis. Hepatology 2003;37:544-50.

47. Bugianesi $E$, Gastaldelli A, Vanni E, et al. Insulin resistance in non-diabetic patients with nonalcoholic fatty liver disease: sites and mechanisms. Diabetologia 2005;48:634-42.

48. Lambert JE, Ramos-Roman MA, Browning JD, Parks EJ. Increased de novo lipogenesis is a distinct characteristic of individuals with nonalcoholic fatty liver disease. Gastroenterology 2014; 146:726-35.

49. Smith GI, Shankaran M, Yoshino M, et al. Insulin resistance drives hepatic de novo lipogenesis in nonalcoholic fatty liver disease. J Clin Invest 2020;130:1453-60.

50. Williams CD, Stengel J, Asike MI, et al. Prevalence of nonalcoholic fatty liver disease and nonalcoholic steatohepatitis among a largely middle-aged population utilizing ultrasound and liver biopsy: a prospective study. Gastroenterology 2011;140:124-31.

51. Yamaguchi K, Yang L, McCall S, et al. Inhibiting triglyceride synthesis improves hepatic steatosis but exacerbates liver damage and fibrosis in obese mice with nonalcoholic steatohepatitis. Hepatology 2007;45:1366-74.

52. Li ZZ, Berk M, Mclntyre TM, Feldstein AE. Hepatic lipid partitioning and liver damage in nonalcoholic fatty liver disease: role of stearoyl-CoA desaturase. J Biol Chem 2009;284: 5637-44.

53. Begriche K, Massart J, Robin MA, Bonnet F, Fromenty B. Mitochondrial adaptations and dysfunctions in nonalcoholic fatty liver disease. Hepatology 2013;58:1497-507.

54. Eslam M, George J. Genetic and epigenetic mechanisms of NASH. Hepatol Int 2016;10: 394-406.

55. Eslam M, George J. Genetic contributions to NAFLD: leveraging shared genetics to uncover systems biology. Nat Rev Gastroenterol Hepatol 2020;17(1):40-52.

56. Romeo S, Kozlitina J, Xing C, et al. Genetic variation in PNPLA3 confers susceptibility to nonalcoholic fatty liver disease. Nat Genet 2008;40:1461-5.

57. Valenti L, Al-Serri A, Daly AK, et al. Homozygosity for the patatin-like phospholipase3/adiponutrin I148M polymorphism influences liver fibrosis in patients with nonalcoholic fatty liver disease. Hepatology 2010;51:1209-17.

58. Smagris E, BasuRay S, Li J, et al. Pnpla3/148M knockin mice accumulate PNPLA3 on lipid droplets and develop hepatic steatosis. Hepatology 2015;61:108-18.

59. Trepo E, Romeo S, Zucman-Rossi J, Nahon P. PNPLA3 gene in liver diseases. J Hepatol 2016;65:399-412.

60. Wang X, Guo M, Wang Q, et al. The patatin-like phospholipase domain containing protein 7 facilitates VLDL secretion by modulating ApoE stability. Hepatology 2020.

61. Palmer CN, Maglio C, Pirazzi C, et al. Paradoxical lower serum triglyceride levels and higher type 2 diabetes mellitus susceptibility in obese individuals with the PNPLA3 148M variant. PloS One 2012;7:e39362. 
62. Kozlitina J, Smagris E, Stender S, et al. Exome-wide association study identifies a TM6SF2 variant that confers susceptibility to nonalcoholic fatty liver disease. Nat Genet 2014;46: 352-6.

63. Mahdessian $\mathrm{H}$, Taxiarchis A, Popov $\mathrm{S}$, et al. TM6SF2 is a regulator of liver fat metabolism influencing triglyceride secretion and hepatic lipid droplet content. Proc Natl Acad Sci U S A 2014;111:8913-8.

64. Mancina RM, Dongiovanni P, Petta S, et al. The MBOAT7-TMC4 Variant rs641738 Increases Risk of Nonalcoholic Fatty Liver Disease in Individuals of European Descent. Gastroenterology 2016;150:1219-30.e6.

65. Luukkonen PK, Zhou Y, Hyotylainen T, et al. The MBOAT7 variant rs641738 alters hepatic phosphatidylinositols and increases severity of non-alcoholic fatty liver disease in humans. $J$ Hepatol 2016;65:1263-5.

66. van Schaftingen E, Vandercammen A, Detheux M, Davies DR. The regulatory protein of liver glucokinase. Adv Enzyme Regul 1992;32:133-48.

67. Beer NL, Tribble ND, McCulloch LJ, et al. The P446L variant in GCKR associated with fasting plasma glucose and triglyceride levels exerts its effect through increased glucokinase activity in liver. Hum Mol Genet 2009;18:4081-8.

68. Perez-Martinez P, Delgado-Lista J, Garcia-Rios A, et al. Glucokinase regulatory protein genetic variant interacts with omega-3 PUFA to influence insulin resistance and inflammation in metabolic syndrome. PloS One 2011;6:e20555.

69. Qi Q, Wu Y, Li H, et al. Association of GCKR rs780094, alone or in combination with GCK rs 1799884, with type 2 diabetes and related traits in a Han Chinese population. Diabetologia 2009;52:834-43.

70. Vaxillaire M, Cavalcanti-Proenca C, Dechaume A, et al. The common P446L polymorphism in GCKR inversely modulates fasting glucose and triglyceride levels and reduces type 2 diabetes risk in the DESIR prospective general French population. Diabetes 2008;57:2253-7.

71. Speliotes EK, Yerges-Armstrong LM, Wu J, et al. Genome-wide association analysis identifies variants associated with nonalcoholic fatty liver disease that have distinct effects on metabolic traits. PLoS Genet 2011;7:e1001324.

72. Santoro N, Caprio S, Pierpont B, Van Name M, Savoye M, Parks EJ. Hepatic De Novo Lipogenesis in Obese Youth Is Modulated by a Common Variant in the GCKR Gene. The J Clin Endocrinol Metab 2015;100:E1125-32.

73. Forouhi NG, Krauss RM, Taubes G, Willett W. Dietary fat and cardiometabolic health: evidence, controversies, and consensus for guidance. BMJ 2018;361:k2139.

74. Malik VS, Li Y, Pan A, et al. Long-Term Consumption of Sugar-Sweetened and Artificially Sweetened Beverages and Risk of Mortality in US Adults. Circulation 2019;139:2113-25.

75. Welsh JA, Sharma AJ, Grellinger L, Vos MB. Consumption of added sugars is decreasing in the United States. Am J Clin Nutr 2011;94:726-34.

76. Austin GL, Ogden LG, Hill JO. Trends in carbohydrate, fat, and protein intakes and association with energy intake in normal-weight, overweight, and obese individuals: 19712006. Am J Clin Nutr 2011;93:836-43.

77. Luukkonen PK, Sadevirta S, Zhou Y, et al. Saturated Fat Is More Metabolically Harmful for the Human Liver Than Unsaturated Fat or Simple Sugars. Diabetes Care 2018;41:1732-9.

78. Jensen T, Abdelmalek MF, Sullivan S, et al. Fructose and sugar: A major mediator of nonalcoholic fatty liver disease. J Hepatol 2018;68:1063-75.

79. Hannou SA, Haslam DE, McKeown NM, Herman MA. Fructose metabolism and metabolic disease. J Clin Invest 2018;128:545-55.

80. Gluchowski NL, Becuwe M, Walther TC, Farese RV, Jr. Lipid droplets and liver disease: from basic biology to clinical implications. Nat Rev Gastroenterol Hepatol 2017;14:343-55.

81. DiStefano JK. Fructose-mediated effects on gene expression and epigenetic mechanisms associated with NAFLD pathogenesis. Cell Mol Life Sci 2020;77(11):2079-90.

82. Chong MF, Fielding BA, Frayn KN. Mechanisms for the acute effect of fructose on postprandial lipemia. Am J Clin Nutr 2007;85:1511-20. 
83. Softic S, Gupta MK, Wang GX, et al. Divergent effects of glucose and fructose on hepatic lipogenesis and insulin signaling. J Clin Invest 2017;127:4059-74.

84. Huard K, Ahn K, Amor P, et al. Discovery of Fragment-Derived Small Molecules for in Vivo Inhibition of Ketohexokinase (KHK). J Med Chem 2017;60:7835-49.

85. Lanaspa MA, Andres-Hernando A, Orlicky DJ, et al. Ketohexokinase $C$ blockade ameliorates fructose-induced metabolic dysfunction in fructose-sensitive mice. J Clin Invest 2018;128: 2226-38.

86. Chiu S, Sievenpiper JL, de Souza RJ, et al. Effect of fructose on markers of non-alcoholic fatty liver disease (NAFLD): a systematic review and meta-analysis of controlled feeding trials. Eur J Clin Nutr 2014;68:416-23.

87. Sobrecases H, Le KA, Bortolotti M, et al. Effects of short-term overfeeding with fructose, fat and fructose plus fat on plasma and hepatic lipids in healthy men. Diabetes Metab 2010;36: 244-6.

88. Le KA, Ith $M$, Kreis $R$, et al. Fructose overconsumption causes dyslipidemia and ectopic lipid deposition in healthy subjects with and without a family history of type 2 diabetes. Am J Clin Nutr 2009;89:1760-5.

89. Kechagias S, Ernersson A, Dahlqvist O, Lundberg P, Lindstrom T, Nystrom FH. Fast-foodbased hyper-alimentation can induce rapid and profound elevation of serum alanine aminotransferase in healthy subjects. Gut 2008;57:649-54.

90. Sevastianova K, Santos A, Kotronen A, et al. Effect of short-term carbohydrate overfeeding and long-term weight loss on liver fat in overweight humans. Am J Clin Nutr 2012;96:727-34.

91. Schwarz JM, Noworolski SM, Erkin-Cakmak A, et al. Effects of Dietary Fructose Restriction on Liver Fat, De Novo Lipogenesis, and Insulin Kinetics in Children With Obesity. Gastroenterology 2017;153:743-52.

92. Khan RS, Bril F, Cusi K, Newsome PN. Modulation of Insulin Resistance in Nonalcoholic Fatty Liver Disease. Hepatology 2019;70:711-24.

93. Rinella ME. Nonalcoholic fatty liver disease: a systematic review. Jama 2015;313:2263-73.

94. Mantovani A, Byrne CD, Bonora E, Targher G. Nonalcoholic Fatty Liver Disease and Risk of Incident Type 2 Diabetes: A Meta-analysis. Diabetes Care 2018;41:372-82.

95. Lomonaco R, Ortiz-Lopez C, Orsak B, et al. Effect of adipose tissue insulin resistance on metabolic parameters and liver histology in obese patients with nonalcoholic fatty liver disease. Hepatology 2012;55:1389-97.

96. Yamashita $\mathrm{H}$, Takenoshita M, Sakurai $\mathrm{M}$, et al. A glucose-responsive transcription factor that regulates carbohydrate metabolism in the liver. Proc Natl Acad Sci U S A 2001;98:9116-21.

97. Seppala-Lindroos A, Vehkavaara S, Hakkinen AM, et al. Fat accumulation in the liver is associated with defects in insulin suppression of glucose production and serum free fatty acids independent of obesity in normal men. J Clin Endocrinol Metab 2002;87:3023-8.

98. Marchesini G, Brizi M, Bianchi G, et al. Nonalcoholic fatty liver disease: a feature of the metabolic syndrome. Diabetes 2001;50:1844-50.

99. Chen SC, Tsai SP, Jhao JY, Jiang WK, Tsao CK, Chang LY. Liver Fat, Hepatic Enzymes, Alkaline Phosphatase and the Risk of Incident Type 2 Diabetes: A Prospective Study of 132,377 Adults. Sci Rep 2017;7:4649.

100. Ballestri S, Zona S, Targher G, et al. Nonalcoholic fatty liver disease is associated with an almost twofold increased risk of incident type 2 diabetes and metabolic syndrome. Evidence from a systematic review and meta-analysis. J Gastroenterol Hepatol 2016;31:936-44.

101. Amaro A, Fabbrini E, Kars M, et al. Dissociation between intrahepatic triglyceride content and insulin resistance in familial hypobetalipoproteinemia. Gastroenterology 2010;139:149-53.

102. Santos RD, Valenti L, Romeo S. Does nonalcoholic fatty liver disease cause cardiovascular disease? Current knowledge and gaps. Atherosclerosis 2019;282:110-20.

103. Francque SM, van der Graaff D, Kwanten WJ. Non-alcoholic fatty liver disease and cardiovascular risk: Pathophysiological mechanisms and implications. J Hepatol 2016;65: $425-43$. 
104. DeFilippis AP, Blaha MJ, Martin SS, et al. Nonalcoholic fatty liver disease and serum lipoproteins: the Multi-Ethnic Study of Atherosclerosis. Atherosclerosis 2013;227:429-36.

105. Siddiqui MS, Fuchs M, Idowu MO, et al. Severity of nonalcoholic fatty liver disease and progression to cirrhosis are associated with atherogenic lipoprotein profile. Clin Gastroenterol Hepatol 2015;13:1000-8.e3.

106. Sonmez A, Nikolic D, Dogru T, et al. Low- and high-density lipoprotein subclasses in subjects with nonalcoholic fatty liver disease. J Clin Lipidol 2015;9:576-82.

107. Adiels M, Taskinen MR, Packard C, et al. Overproduction of large VLDL particles is driven by increased liver fat content in man. Diabetologia 2006;49:755-65.

108. Cassader M, Gambino R, Musso G, et al. Postprandial triglyceride-rich lipoprotein metabolism and insulin sensitivity in nonalcoholic steatohepatitis patients. Lipids 2001;36:1117-24.

109. Taskinen MR. Diabetic dyslipidaemia: from basic research to clinical practice. Diabetologia 2003;46:733-49.

110. Adiels M, Olofsson SO, Taskinen MR, Boren J. Overproduction of very low-density lipoproteins is the hallmark of the dyslipidemia in the metabolic syndrome. Arterioscler Thromb Vasc Biol 2008;28:1225-36.

111. Do R, Willer CJ, Schmidt EM, et al. Common variants associated with plasma triglycerides and risk for coronary artery disease. Nat Genet 2013;45:1345-52.

112. Ross R. Atherosclerosis--an inflammatory disease. N Engl J Med 1999;340:115-26.

113. Hansson GK. Inflammation, atherosclerosis, and coronary artery disease. N Engl J Med 2005;352:1685-95.

114. Crespo J, Cayon A, Fernandez-Gil P, et al. Gene expression of tumor necrosis factor alpha and TNF-receptors, p55 and p75, in nonalcoholic steatohepatitis patients. Hepatology 2001;34:1158-63.

115. Wieckowska A, Papouchado BG, Li Z, Lopez R, Zein NN, Feldstein AE. Increased hepatic and circulating interleukin-6 levels in human nonalcoholic steatohepatitis. Am J Gastroenterol 2008;103:1372-9.

116. Kasumov T, Edmison JM, Dasarathy S, Bennett C, Lopez R, Kalhan SC. Plasma levels of asymmetric dimethylarginine in patients with biopsy-proven nonalcoholic fatty liver disease. Metabolism 2011;60:776-81.

117. Pacana T, Cazanave S, Verdianelli A, et al. Dysregulated Hepatic Methionine Metabolism Drives Homocysteine Elevation in Diet-Induced Nonalcoholic Fatty Liver Disease. PloS One 2015;10:e0136822.

118. Targher G. Relationship between high-sensitivity C-reactive protein levels and liver histology in subjects with non-alcoholic fatty liver disease. J Hepatol 2006;45:879-81; author reply 81-2.

119. Haukeland JW, Damas JK, Konopski Z, et al. Systemic inflammation in nonalcoholic fatty liver disease is characterized by elevated levels of CCL2. J Hepatol 2006;44:1167-74.

120. Mirea AM, Tack CJ, Chavakis T, Joosten LAB, Toonen EJM. IL-1 Family Cytokine Pathways Underlying NAFLD: Towards New Treatment Strategies. Trends Mol Med 2018;24:458-71.

121. Ridker PM, Everett BM, Thuren T, et al. Antiinflammatory Therapy with Canakinumab for Atherosclerotic Disease. N Engl J Med 2017;377:1119-31.

122. O'Connor NT, Cederholm-Williams S, Copper S, Cotter L. Hypercoagulability and coronary artery disease. Br Heart J 1984;52:614-6.

123. Loeffen $R$, Spronk HM, ten Cate $H$. The impact of blood coagulability on atherosclerosis and cardiovascular disease. J Thromb Haemost 2012;10:1207-16.

124. Thuy $S$, Ladurner $R$, Volynets $V$, et al. Nonalcoholic fatty liver disease in humans is associated with increased plasma endotoxin and plasminogen activator inhibitor 1 concentrations and with fructose intake. J Nutr 2008;138:1452-5.

125. Alessi MC, Bastelica D, Mavri A, et al. Plasma PAl-1 levels are more strongly related to liver steatosis than to adipose tissue accumulation. Arterioscler Thromb Vasc Biol 2003;23: $1262-8$.

126. Kotronen A, Joutsi-Korhonen L, Sevastianova K, et al. Increased coagulation factor VIII, IX, XI and XII activities in non-alcoholic fatty liver disease. Liver Int 2011;31:176-83. 
127. Tripodi A, Fracanzani AL, Primignani M, et al. Procoagulant imbalance in patients with nonalcoholic fatty liver disease. J Hepatol 2014;61:148-54.

128. Brownlee M. Biochemistry and molecular cell biology of diabetic complications. Nature 2001;414:813-20.

129. Song C, Burgess S, Eicher JD, O'Donnell CJ, Johnson AD. Causal Effect of Plasminogen Activator Inhibitor Type 1 on Coronary Heart Disease. J Am Heart Assoc 2017;6.

130. Meex RCR, Watt MJ. Hepatokines: linking nonalcoholic fatty liver disease and insulin resistance. Nat Rev Endocrinol 2017;13:509-20.

131. Stefan N, Haring HU. The role of hepatokines in metabolism. Nature reviews Endocrinology 2013;9:144-52.

132. Lin X, Braymer HD, Bray GA, York DA. Differential expression of insulin receptor tyrosine kinase inhibitor (fetuin) gene in a model of diet-induced obesity. Life Sci 1998;63:145-53.

133. Stefan $\mathrm{N}$, Hennige AM, Staiger $\mathrm{H}$, et al. Alpha2-Heremans-Schmid glycoprotein/fetuin-A is associated with insulin resistance and fat accumulation in the liver in humans. Diabetes Care 2006;29:853-7.

134. Reinehr T, Roth CL. Fetuin-A and its relation to metabolic syndrome and fatty liver disease in obese children before and after weight loss. J Clin Endocrinol Metab 2008;93:4479-85.

135. Haukeland JW, Dahl TB, Yndestad A, et al. Fetuin A in nonalcoholic fatty liver disease: in vivo and in vitro studies. Eur J Endocrinol 2012;166:503-10.

136. Dogru T, Genc H, Tapan S, et al. Plasma fetuin-A is associated with endothelial dysfunction and subclinical atherosclerosis in subjects with nonalcoholic fatty liver disease. Clin Endocrinol 2013;78:712-7.

137. Rittig $K$, Thamer $C$, Haupt A, et al. High plasma fetuin-A is associated with increased carotid intima-media thickness in a middle-aged population. Atherosclerosis 2009;207:341-2.

138. Weikert C, Stefan N, Schulze MB, et al. Plasma fetuin-a levels and the risk of myocardial infarction and ischemic stroke. Circulation 2008;118:2555-62.

139. Fisher E, Stefan N, Saar K, et al. Association of AHSG gene polymorphisms with fetuin-A plasma levels and cardiovascular diseases in the EPIC-Potsdam study. Circ Cardiovasc Genet 2009;2:607-13.

140. Pal D, Dasgupta S, Kundu R, et al. Fetuin-A acts as an endogenous ligand of TLR4 to promote lipid-induced insulin resistance. Nat Med 2012;18:1279-85.

141. Dasgupta S, Bhattacharya S, Biswas A, et al. NF-kappaB mediates lipid-induced fetuin-A expression in hepatocytes that impairs adipocyte function effecting insulin resistance. Biochem J 2010;429:451-62.

142. Korenblat KM, Fabbrini E, Mohammed BS, Klein S. Liver, muscle, and adipose tissue insulin action is directly related to intrahepatic triglyceride content in obese subjects. Gastroenterology 2008;134:1369-75.

143. Merino J, Leong A, Posner DC, et al. Genetically Driven Hyperglycemia Increases Risk of Coronary Artery Disease Separately From Type 2 Diabetes. Diabetes Care 2017;40:687-93.

144. Magkos F, Mittendorfer B. Stable isotope-labeled tracers for the investigation of fatty acid and triglyceride metabolism in humans in vivo. Clin Lipidol 2009;4:215-30.

145. Holmes MV, Ala-Korpela M, Smith GD. Mendelian randomization in cardiometabolic disease: challenges in evaluating causality. Nat Rev Cardiol 2017;14:577-90.

146. Smith GD, Ebrahim S. 'Mendelian randomization': can genetic epidemiology contribute to understanding environmental determinants of disease? Int J Epidemiol 2003;32:1-22.

147. Orho-Melander M, Melander O, Guiducci $C$, et al. Common missense variant in the glucokinase regulatory protein gene is associated with increased plasma triglyceride and Creactive protein but lower fasting glucose concentrations. Diabetes 2008;57:3112-21.

148. Gimbrone MA, Jr., Garcia-Cardena G. Endothelial Cell Dysfunction and the Pathobiology of Atherosclerosis. Circ Res 2016;118:620-36.

149. Bertola A, Park O, Gao B. Chronic plus binge ethanol feeding synergistically induces neutrophil infiltration and liver injury in mice: a critical role for E-selectin. Hepatology 2013;58:1814-23. 
Chapter 1

150. Ali M, Rellos P, Cox TM. Hereditary fructose intolerance. J M 
Genetic studies to elucidate the relationship between nonalcoholic fatty liver disease and cardiovascular disease 



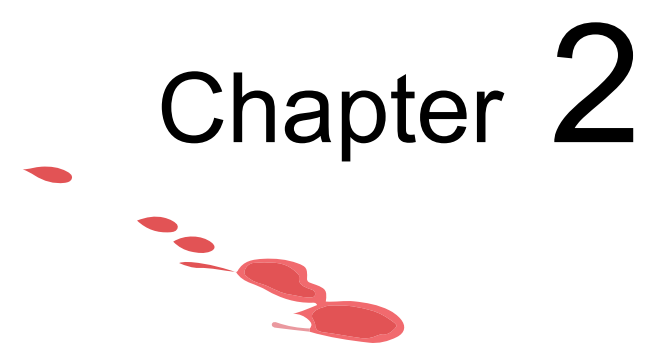

\section{Interaction between a genetic variant in GCKR and environmental factors}

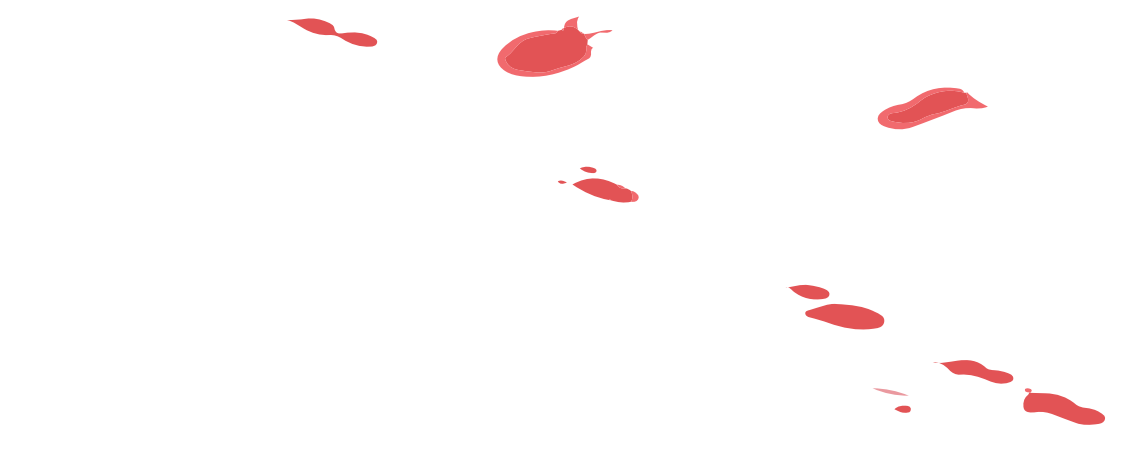

A common gene variant in glucokinase regulatory protein interacts with glucose metabolism on diabetic dyslipidemia: the combined CODAM and Hoorn studies

Nynke Simons, Jacquefine M.Dekker, Marleen M.J.van Greevenbroek, Giel Nijpels, Leen M. 't Hart, Carla J.H. van der Kallen, Casper G. Schalkwijk, Nicolaas C. Schaper, Coen D.A. Stehouwer, Martijn C.G.J. Brouwers 


\section{Abstract}

\section{Background}

Small molecules that disrupt the binding between glucokinase and glucokinase regulatory protein are potential new glucose-lowering targets. They stimulate hepatic glucose disposal by increasing glucokinase activity in the liver. It can, however, be anticipated that increased hepatic glucokinase activity might be accompanied by the development of hypertriglyceridemia, particularly in type 2 diabetes. We examined whether the strength of association between rs1260326 - a common, functional genetic variant in glucokinase regulatory protein - and plasma lipids is affected by glucose metabolism.

\section{Methods}

Rs1260326 was genotyped in subjects with normal glucose metabolism $(n=497)$, impaired glucose metabolism $(n=256)$ and patients with type 2 diabetes $(n=351)$ in the combined Hoorn and CODAM studies.

\section{Results}

The strength of association between the rs1260326 minor T-allele and plasma triglycerides increased from normal glucose metabolism to impaired glucose metabolism to type 2 diabetes ( $p$ for interaction=0.002). The inverse relation between rs1260326 and plasma HDL cholesterol was again most prominent in type 2 diabetes ( $p$ for interaction=0.004). Similar trends were observed when the Hoorn and CODAM cohorts were analyzed separately. Comparable results were obtained when glucose metabolism strata were replaced by continuous indices of glucose metabolism, i.e., $\mathrm{HbA} 1 \mathrm{c}$ and fasting plasma glucose.

\section{Conclusions}

In conclusion, these findings illustrate that common genetics variants, such as rs1260326, can have substantial effect sizes when they are studied in specific populations, such as type 2 diabetes. Moreover, our results shed light on potential side-effects of small molecule disruptors of the glucokinaseglucokinase regulatory protein complex, especially when glucose control is suboptimal. 


\section{Background}

Glucokinase is the principal, rate-limiting enzyme in glycolysis that phosphorylates glucose in the pancreas and liver. It is therefore an important regulator of insulin secretion and hepatic glucose disposal, respectively. ${ }^{1}$ Loss of function mutations in the glucokinase gene result in maturity-onset diabetes of the young type 2 (MODY2). ${ }^{2,3}$ Given its major role in glucose metabolism, efforts have been undertaken to develop glucokinase activators as a potential new class of glucose-lowering drugs. ${ }^{4}$ Clinical studies have demonstrated that these systemic activators are capable of lowering plasma glucose levels, albeit at the expense of hypoglycemia. ${ }^{5,6}$ This undesired side-effect could be circumvented by organ-specific glucokinase activators, which only act in the liver, not in the pancreas. ${ }^{\text {? }}$

Lloyd and colleagues recently reported a different approach to stimulate hepatic glucokinase activity only. ${ }^{8}$ They developed a small molecule that disrupts the binding between glucokinase and glucokinase regulatory protein, a liver-specific protein that binds and inactivates glucokinase. Disruption of the glucokinase-glucokinase regulatory protein complex facilitates migration of glucokinase to the cytosolic space where glycolysis and consequently hepatic glucose disposal are stimulated. ${ }^{1}$ Mice treated with these small molecules indeed displayed lower plasma glucose levels, without hypoglycemia. ${ }^{8}$

It can, however, be anticipated that an increased hepatic glucose disposal will affect intrahepatic glucose metabolism, such as stimulation of glycogenesis as well as de novo lipogenesis. This may accelerate the development of nonalcoholic fatty liver disease (NAFLD) and hypertriglyceridemia. ${ }^{9}$ Clinical studies with small-molecule glucokinase-glucokinase regulatory protein disruptors are therefore eagerly awaited.

Meanwhile, lessons can be learned from humans carrying genetic variants that are known to encode glucokinase regulatory protein (GCKR) that binds glucokinase less effectively. ${ }^{10}$ We recently reviewed the metabolic effects of such common, functional variants in GCKR and showed that the rs780094 and rs1260326 minor alleles are associated with not only decreased plasma glucose levels, but also increased de novo lipogenesis, NAFLD and plasma triglycerides. ${ }^{9}$ Notably, many of these studies have been carried out in individuals with normal glucose metabolism. Based on enzyme kinetics and expression, it can be anticipated that the metabolic consequences of common variants in GCKR are different in patients with type 2 diabetes, although the exact effects may be difficult to predict. Glucokinase has a relatively low affinity 
for glucose $\left(\mathrm{S}_{0.5} \approx 7.5 \mathrm{mmol} / \mathrm{L}\right)^{11}$, implicating that it is more active when plasma glucose levels are within the diabetic range. ${ }^{12}$ This would suggest that the effect size of the GCKR minor allele variant on plasma triglycerides is more pronounced in patients with type 2 diabetes compared to individuals without type 2 diabetes. On the other hand, glucokinase expression has been shown to be reduced in liver biopsies derived from type 2 diabetic humans. ${ }^{13}$

The aim of the current study was therefore to examine whether the association between GCKR and plasma lipids differs between individuals with normal glucose metabolism, impaired glucose metabolism and type 2 diabetes.

\section{Methods}

\section{Study population}

Study participants were derived from the Hoorn follow-up cohort and the Cohort on Diabetes and Atherosclerosis Maastricht (CODAM). In brief, Hoorn and CODAM are prospective cohorts designed to study determinants and (cardiovascular) complications of type 2 diabetes. The original Hoorn study was conducted in a random sample $(n=2484)$ of the general population of Hoorn, the Netherlands, between 1989 and $1992 .{ }^{14}$ In 2000, a follow-up study was performed in a subset of participants with normal and impaired glucose metabolism and type 2 diabetes $\left(n=836\right.$ in total). ${ }^{15,16}$ The CODAM study was carried out in individuals with elevated risk for type 2 diabetes $(n=574)$ from the Dutch Monitoring Project for Cardiovascular Diseases (MORGEN), between 1999 and $2000 .^{17-19}$

Since comparable research protocols have been used in the Hoorn and CODAM study, both cohorts were merged into one larger cohort in this and previous studies. ${ }^{20-23}$ The present study consisted of 1286 individuals who were available for genotyping. The Hoorn and CODAM study did differ in terms of lipid-modifying medication use, i.e., participants of the CODAM study were asked to stop lipid-modifying medication two weeks prior to their visit (of whom 40 did not stop), whereas subjects in the Hoorn study were allowed to continue their lipid-modifying medication. Since plasma lipids were the main outcome variable of this study, all individuals from the Hoorn and CODAM study taking lipid-modifying medication at time of visit were excluded from analyses $(n=153$ in total). 
Written informed consent was given by all participants. Approval for the Hoorn and CODAM study was provided by the Human Investigations Review Committee of VU University Medical Center and Maastricht University Medical Center, respectively.

\section{Measurements}

Height was determined standing upright against a stadiometer and weight was measured using an electronic weight scale without shoes. BMI was calculated as weight divided by height squared. Waist circumference was ascertained with a measuring-tape at the level of umbilicus. Blood samples were drawn after an overnight fast for measurement of glycated hemoglobin ( $\mathrm{HbA} 1 \mathrm{c})$, plasma glucose, insulin, total cholesterol, high-density lipoprotein (HDL) cholesterol and triglycerides, as described elsewhere. ${ }^{24,25}$ Low-density lipoprotein (LDL) cholesterol was calculated by the Friedewald formula. The degree of insulin resistance (HOMA2-IR) was estimated with the HOMA2 calculator (http://www.dtu.ox.ac.uk). Participants underwent a standard 75 gram oral glucose tolerance test (OGTT) and were subsequently divided into three groups; normal glucose metabolism, impaired glucose metabolism (which comprised impaired fasting glycemia and impaired glucose tolerance) and type 2 diabetes, according to the World Health Organisation (WHO) criteria. ${ }^{26}$ Of the 1286 individuals who were genotyped, an OGTT was not performed in 11 participants; they were therefore excluded from analyses.

\section{Genotyping}

Genotyping of rs1260326 - which has been shown to be a functional variant that is in strong linkage disequilibrium with $\mathrm{rs} 780094^{10,27}$ - was done in the CODAM study as part of a genome wide association study (GWAS) array, with the use of a HumanOmniExpress BeadChip assay (Illumina, San Diego, CA, USA). In the Hoorn study, a validated Invitrogen taqman assay was used (ThermoFisher, Waltham, Massachusetts, USA). Genotyping was successful in $98 \%(695 / 712)$ and $99 \%(573 / 574)$ of the Hoorn and CODAM samples, respectively. The minor allele frequency (T-allele) was 0.38 in the overall population $(0.38,0.39$ and 0.39 in the normal glucose metabolism, impaired glucose metabolism and type 2 diabetes subgroups, respectively). Genotype distribution was in Hardy-Weinberg equilibrium ( $p=0.76$; Chi-Square test).

\section{Statistics}

Data are presented as mean \pm SD or as median (interquartile range) in case of non-normal distribution. Non-normally distributed variables were log- 
transformed before further analyses. Differences in characteristics between subgroups (i.e., normal glucose metabolism, impaired glucose metabolism and type 2 diabetes) were analyzed using linear or logistic regression, with adjustments for age, sex and cohort (i.e., the Hoorn and CODAM study). Associations between the rs1260326 minor T-allele and plasma lipid levels were analyzed using linear regression, with adjustments for age, sex and cohort, and stratified by glucose metabolism subgroups. An additive mode of inheritance was assumed, based on previous reports. ${ }^{28}$ To investigate whether the strength of the association between the rs1260326 minor T-allele and plasma lipids differed between the three subgroups of glucose metabolism, interaction terms (i.e., rs $1260326^{*}$ glucose metabolism subgroups) were used. Interactions were considered statistically significant at $p<0.10$. The remaining results were considered statistically significant at $p<0.05$. All statistical analyses were carried out by the IBM Statistical Package of Social Sciences (SPSS) version 22 for Windows (SPSS Inc, Chicago, III, USA).

\section{Results}

Characteristics of overall study population and glucose metabolism subgroups

General characteristics of the overall study population, as well as stratified by levels of glucose metabolism, are displayed in Table 2.1. After adjustment for age, sex and cohort, individuals with impaired glucose metabolism and type 2 diabetes displayed higher levels of $\mathrm{HbA} 1 \mathrm{c}$, fasting plasma glucose, insulin and triglycerides, HOMA2-IR, BMI, waist circumference, systolic and diastolic blood pressure, and use of blood pressure-lowering medication when compared to individuals with normal glucose metabolism. In contrast, plasma HDL cholesterol concentrations decreased as glucose tolerance deteriorated. 
Table 2.1 Characteristics of the overall population and stratified by glucose metabolism stage.

\begin{tabular}{|c|c|c|c|c|}
\hline & $\begin{array}{l}\text { Overall } \\
(n=1104)\end{array}$ & $\begin{array}{c}\text { Normal glucose } \\
\text { metabolism } \\
(n=497)\end{array}$ & $\begin{array}{c}\text { Impaired glucose } \\
\text { metabolism } \\
(n=256)\end{array}$ & $\begin{array}{c}\text { Type } 2 \text { diabetes } \\
(n=351)\end{array}$ \\
\hline Male/Female & $611 / 493$ & $271 / 226$ & $144 / 112$ & $196 / 155$ \\
\hline Age $(y)$ & $64 \pm 9$ & $63 \pm 9$ & $66 \pm 8^{*}$ & $65 \pm 8$ \\
\hline BMI $\left(\mathrm{kg} / \mathrm{m}^{2}\right)$ & $28.1 \pm 4.3$ & $26.9 \pm 3.7$ & $28.2 \pm 4.3^{*}$ & $29.6 \pm 4.6^{\star \dagger}$ \\
\hline Waist circumference $(\mathrm{cm})$ & $98 \pm 12$ & $94 \pm 11$ & $99 \pm 11^{*}$ & $103 \pm 12^{* \dagger}$ \\
\hline Fasting glucose (mmol/L) & $5.8(5.3-6.7)$ & $5.3(5.1-5.6)$ & $6.1(5.6-6.4)^{*}$ & $7.3(6.7-8.3)^{\star \dagger}$ \\
\hline $\mathrm{HbA} 1 \mathrm{c}(\%)$ & $6.0 \pm 0.8$ & $5.7 \pm 0.4$ & $5.8 \pm 0.4^{*}$ & $6.7 \pm 1.0^{\star \dagger}$ \\
\hline $\mathrm{HbA} 1 \mathrm{c}(\mathrm{mmol} / \mathrm{mol})$ & $42 \pm 8.5$ & $38 \pm 4.6$ & $40 \pm 4.4^{*}$ & $49 \pm 10.6^{\star \dagger}$ \\
\hline Insulin (pmol/L) & $65(45-99)$ & $53(39-74)$ & $69(49-99)^{*}$ & $90(62-131)^{\star \dagger}$ \\
\hline HOMA2-IR & $1.3(0.9-1.9)$ & $1.0(0.7-1.4)$ & $1.3(1.0-1.9)^{*}$ & $1.8(1.3-2.7)^{\star \dagger}$ \\
\hline Glucose lowering medication (\% yes) & 10 & 0 & 0.8 & $31^{* \dagger}$ \\
\hline Total cholesterol (mmol/L) & $5.5 \pm 1.0$ & $5.5 \pm 1.0$ & $5.6 \pm 1.0$ & $5.5 \pm 1.1^{\dagger}$ \\
\hline LDL cholesterol (mmol/L) & $3.5 \pm 0.9$ & $3.5 \pm 0.9$ & $3.6 \pm 0.9$ & $3.4 \pm 0.9^{* \dagger}$ \\
\hline HDL cholesterol (mmol/L) & $1.3 \pm 0.4$ & $1.4 \pm 0.4$ & $1.3 \pm 0.4^{*}$ & $1.2 \pm 0.3^{*^{\dagger}}$ \\
\hline Triglycerides (mmol/L) & $1.4(1.0-1.9)$ & $1.2(0.9-1.6)$ & $1.4(1.0-2.0)^{*}$ & $1.6(1.2-2.2)^{\star \dagger}$ \\
\hline Systolic blood pressure $(\mathrm{mmHg})$ & $142 \pm 20$ & $137 \pm 19$ & $144 \pm 19^{*}$ & $147 \pm 20^{* \dagger}$ \\
\hline Diastolic blood pressure $(\mathrm{mmHg})$ & $83 \pm 10$ & $81 \pm 10$ & $83 \pm 11^{*}$ & $85 \pm 10^{*}$ \\
\hline Antihypertensive medication ( $\%$ yes) & 35 & 24 & $36^{*}$ & $50^{\star \dagger}$ \\
\hline Smoking (\% yes) & 18 & 19 & 18 & 16 \\
\hline
\end{tabular}

Data are expressed as mean \pm SD or medians (interquartile range). ${ }^{*} p<0.05$ versus normal glucose metabolism; ${ }^{\dagger} p<0.05$ versus impaired glucose metabolism. Analyzed with linear and logistic regression with adjustment for age, sex and cohort (Hoorn/CODAM study).

\section{Association between the rs1260326 minor T-allele and plasma lipid levels in glucose metabolism subgroups}

In the overall population, the rs1260326 minor T-allele was significantly associated with plasma triglycerides, but not with plasma total cholesterol, LDL cholesterol and HDL cholesterol levels (Table 2.2). There was no gene-age or gene-gender interaction on these plasma lipid levels (data not shown). In contrast, stratification by glucose metabolism stage clearly demonstrated that the strength of association between the rs1260326 minor T-allele and plasma triglycerides increased from normal glucose metabolism to impaired glucose metabolism to type 2 diabetes (unstandardized beta: $0.006,0.018$ and 0.067 respectively; $p$ for interaction $=0.002$, Table 2.2). Furthermore, it also showed a significant, inverse association of the minor T-allele with plasma HDL cholesterol in the type 2 diabetes subgroup as well as a significant interaction ( $p$ for interaction $=0.004$, Table 2.2). Additional adjustment for waist circumference (or BMI) and plasma insulin (or HOMA2-IR) did not essentially change these outcomes (data not shown). An interaction was not observed when fasting plasma glucose levels were used as an outcome parameter $(p=0.45)$. 


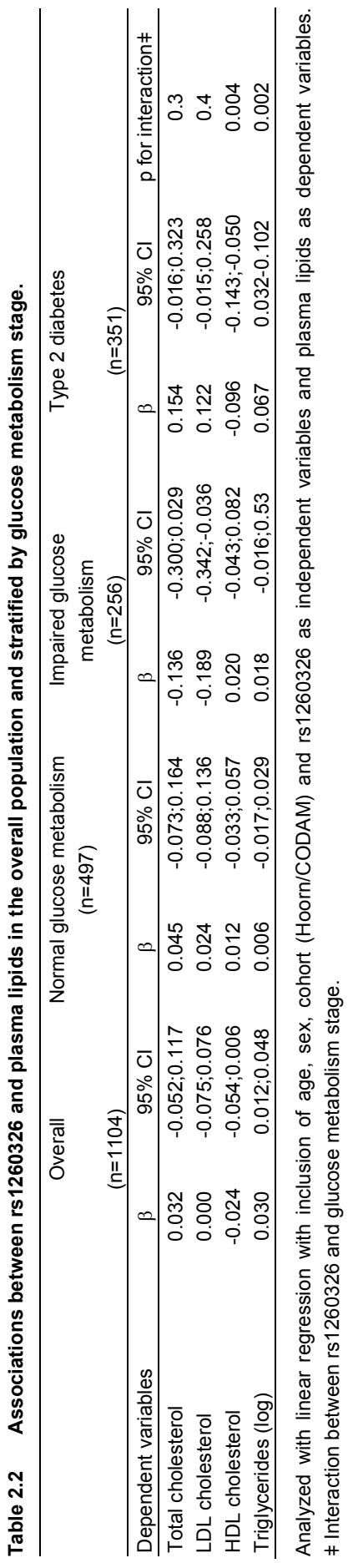


Of note, almost similar results, albeit less significant, were observed when these analyses were repeated in the Hoorn and CODAM study separately ( $p$ for interaction on plasma triglycerides $=0.017$ and 0.167 respectively and $p$ for interaction on plasma HDL cholesterol $=0.042$ and 0.026 respectively). Furthermore, sensitivity analyses to evaluate the impact of exclusion of individuals taking lipid-modifying medication $(n=153)$ yielded comparable outcomes ( $p$ for interaction $=0.013$ and 0.005 for plasma triglycerides and HDL cholesterol, respectively; $n=1257$ ).

\section{Association between plasma triglycerides and HDL cholesterol and continuous metabolic traits stratified by rs 1260326}

Replacement of glucose metabolism strata by continuous indices of glucose metabolism, i.e., fasting plasma glucose and $\mathrm{HbA} 1 \mathrm{c}$ levels, also yielded significant interactions with the rs1260326 minor T-allele on plasma triglycerides ( $p$ for interaction $=0.002$ and 0.01 respectively, Table 2.3, Figure 2.1, panel A). In contrast, we did not observe a statistically significant interaction between other metabolic parameters (BMI, waist circumference, plasma insulin and HOMA2-IR) and rs1260326 on plasma triglycerides, although the strength of association between the anthropometric variables and plasma triglycerides increased from CC to TT carriers ( $p$ for interaction $=0.13$ for both waist circumference and BMI, Table 2.3).

Comparable results were observed for plasma HDL cholesterol levels (Table 2.3, Figure 2.1, panel B).
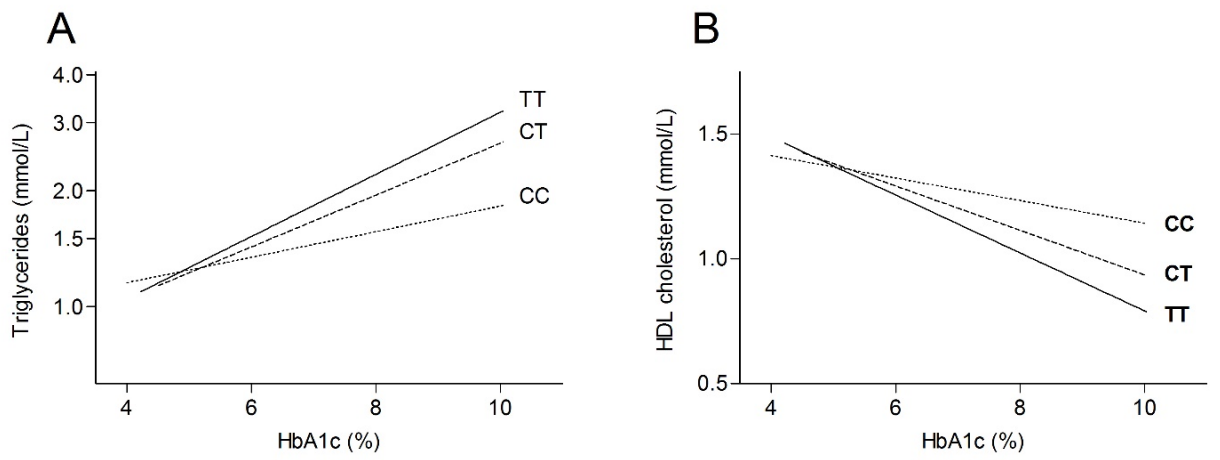

Figure 2.1 Association between plasma HbA1c and plasma triglycerides (panel A) and HDL cholesterol levels (panel B), stratified by rs1260326. Plasma triglycerides are presented on a log scale. 


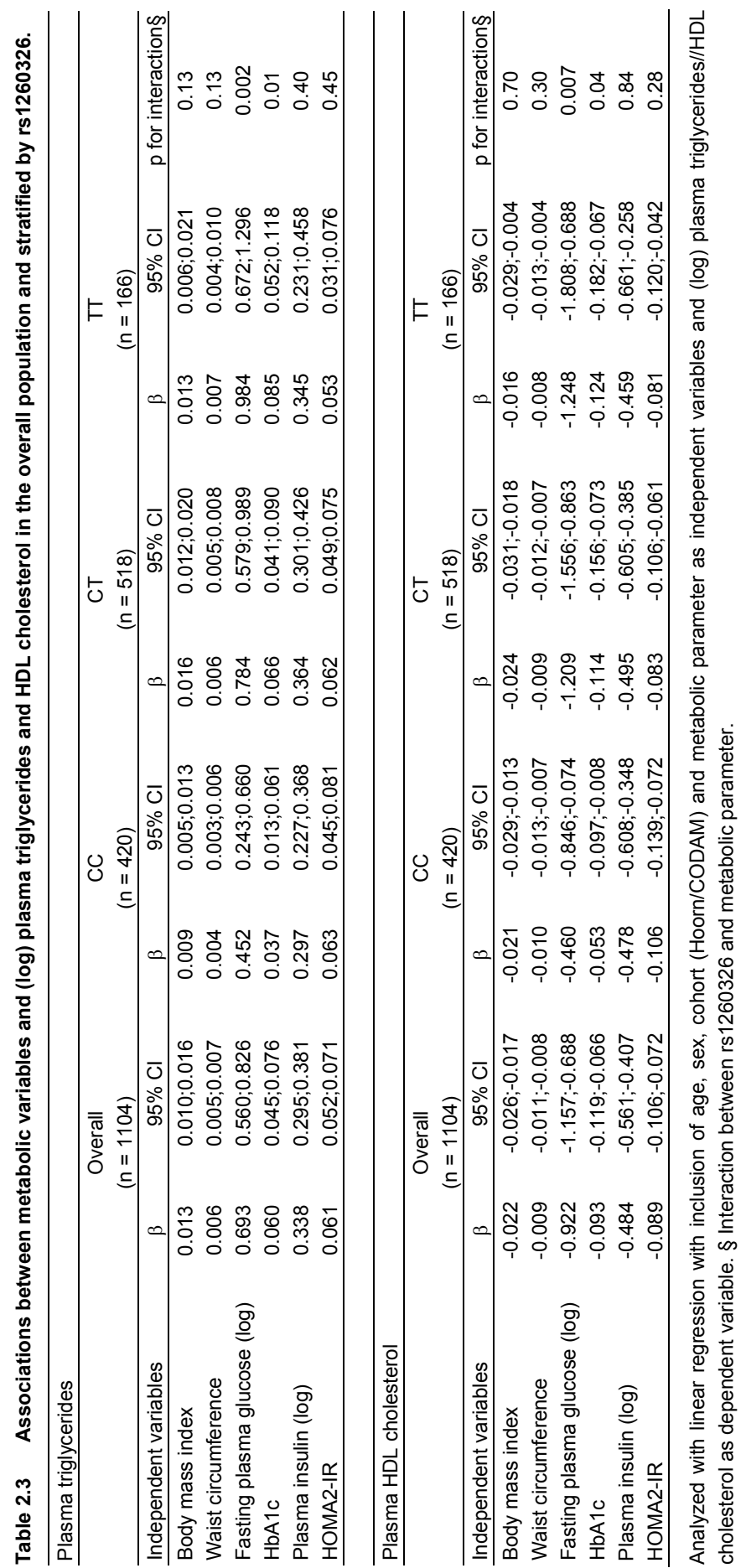




\section{Discussion}

The current study extends previous findings on the relation between GCKR and plasma lipids by demonstrating that rs 1260326 interacts with indices of glucose metabolism, i.e., glucose tolerance state, fasting plasma glucose and $\mathrm{HbA} 1 \mathrm{c}$ levels, on plasma triglycerides and HDL cholesterol, two prominent features of diabetic dyslipidemia. ${ }^{29}$ Patients with moderately controlled type 2 diabetes (HbA1c $8.0 \%$ [64 mmol/mol]) carrying two T-alleles displayed higher plasma triglycerides levels than homozygous carriers of the C-allele $(2.2 \mathrm{mmol} / \mathrm{L}$ versus $1.6 \mathrm{mmol} / \mathrm{L}$ ), whereas no differences were observed in healthy individuals (as illustrated in Figure 2.1, panel A).

Although such an interaction has not been reported before, there are studies that corroborate our findings. In a meta-analysis, Orho-Melander et al. convincingly demonstrated that the minor T-allele of rs780094, which is in strong linkage disequilibrium with rs1260326, is associated with plasma triglycerides. ${ }^{27}$ Of interest, the authors noted that the difference in plasma triglycerides between homozygous carriers of the $\mathrm{C}$ - and T-allele was substantially greater in a cohort that consisted of patients with type 2 diabetes only, i.e., the Skania Diabetes 2000 Registry (delta triglycerides: $0.54 \mathrm{mmol} / \mathrm{L}$ ), in comparison to the general population, e.g., the Malmö Preventive Project (delta triglycerides: $0.11 \mathrm{mmol} / \mathrm{L}$ ) ${ }^{30}$ In addition, Cole and colleagues recently showed that rs1260326 interacts with BMI on plasma lipids. ${ }^{31}$ The present study failed to replicate this observation, but it should be noted that the $p$-value for interaction was 0.13 . The fact that indices of glucose metabolism displayed a more significant interaction with rs 1260326 strongly suggests that glucose metabolism is more closely linked to the pathophysiological pathway underlying these interactions than obesity per se.

Although this pathway has not been elucidated, previous stable isotope studies suggest a prominent role for hepatic de novo lipogenesis from glucose. First, Adiels and colleagues showed that the production of triglycerides-rich very-lowdensity lipoprotein (VLDL) particles is associated with plasma glucose levels ${ }^{32}$, which is in line with the currently observed associations between indices of glucose metabolism and plasma triglycerides. Furthermore, as glucose tolerance deteriorates, de novo lipogenesis becomes a more important source for VLDL production. ${ }^{33}$ In theory, increased metabolic flux - as a consequence of decreased binding of glucokinase regulatory protein to glucokinase - would further drive de novo lipogenesis by providing glucose-6-phosphate to be converted to fatty acids. In statistical terms, this biological mechanism would be reflected by an interaction between indices of glucose metabolism and 
rs1260326. Indeed, individuals carrying the rs1260326 minor T-allele are characterized by increased de novo lipogenesis when compared to homozygous carriers of the $\mathrm{C}$-allele. ${ }^{34}$ Finally, the negative interaction between the rs1260326 minor T-allele and indices of glucose metabolism with plasma $\mathrm{HDL}$ cholesterol is most likely accounted for by the well-documented, negative association between plasma triglycerides and HDL cholesterol levels as a consequence of cholesteryl ester transfer protein (CETP) mediated exchange of cholesteryl esters and triglycerides between VLDL and HDL particles. ${ }^{29}$

The outcomes of this study have several clinically relevant consequences. First, although it is a well-accepted rule of thumb that genetic variants with a high minor allele frequency, such as rs1260326, generally have small effect sizes $^{35}$, the current study clearly demonstrates that common genetic variants can have substantial effect sizes when they are studied in specific populations, such as in type 2 diabetes. In fact, the difference in plasma triglycerides between homozygous type 2 diabetic carriers of the $\mathrm{C}$ - and T-allele was identical to the therapeutic effects of fenofibrate in patients with diabetic dyslipidemia in the Action to Control Cardiovascular Risk in Diabetes (ACCORD) study ${ }^{36}$ These observations also raise the possibility of undesired side-effects of small molecule disruptors of the glucokinase-glucokinase regulatory protein complex. Previous genetic studies have shown to be instrumental in forecasting the clinical effects of newly developed drugs. One illustrative example are genetic variants in proprotein subtilisin kexin type 9 (PCSK9), which have correctly predicted the beneficial LDL cholesterollowering and cardiovascular disease-reducing effects of PCSK9 antagonists. ${ }^{37,38}$ Our study suggests that the glucose-lowering effects of small molecule disruptors of the glucokinase-glucokinase regulatory protein complex will be accompanied by aggravation of dyslipidemia, in particular when glucose regulation is suboptimal. This will seriously limit their applicability in type 2 diabetes treatment and therefore warrants special attention when this new group of drugs is introduced to human subjects in phase I and II studies.

Although the results from this study deserve replication, we believe that the observed interactions are unlikely the consequence of a type I statistical error. First, similar interactions between rs 1260326 and glucose metabolism stage on plasma triglycerides and HDL cholesterol were found when the Hoorn and CODAM cohorts were analyzed separately. Furthermore, the hitherto mentioned, previous genetic reports on GCKR and the biological plausibility further support the validity of the current observations. 
In conclusion, we demonstrated that the strength of association between a common genetic variant in GCKR with plasma triglycerides and HDL cholesterol is stronger in type 2 diabetic patients than healthy controls. These findings illustrate the added value of studying the effect sizes of common genetic variants in selected study populations, such as type 2 diabetes, and question the applicability of small molecule disruptors of the glucokinaseglucokinase regulatory protein complex in type 2 diabetes. 


\section{Literature}

1. Hale C, Lloyd DJ, Pellacani A, Veniant MM. Molecular targeting of the GK-GKRP pathway in diabetes. Expert Opin Ther Targets 2015;19:129-39.

2. Froguel $P$, Vaxillaire $M$, Sun $F$, et al. Close linkage of glucokinase locus on chromosome $7 p$ to early-onset non-insulin-dependent diabetes mellitus. Nature 1992;356:162-4.

3. Hattersley AT, Turner RC, Permutt MA, et al. Linkage of type 2 diabetes to the glucokinase gene. Lancet 1992;339:1307-10.

4. Grimsby J, Sarabu R, Corbett WL, et al. Allosteric activators of glucokinase: potential role in diabetes therapy. Science 2003;301:370-3.

5. Meininger GE, Scott R, Alba M, et al. Effects of MK-0941, a novel glucokinase activator, on glycemic control in insulin-treated patients with type 2 diabetes. Diabetes Care 2011;34: 2560-6.

6. Wilding JP, Leonsson-Zachrisson M, Wessman C, Johnsson E. Dose-ranging study with the glucokinase activator AZD1656 in patients with type 2 diabetes mellitus on metformin. Diabetes Obes Metab 2013;15:750-9.

7. Pfefferkorn JA, Guzman-Perez A, Litchfield J, et al. Discovery of (S)-6-(3-cyclopentyl-2-(4(trifluoromethyl)-1H-imidazol-1-yl)propanamido)nicotini c acid as a hepatoselective glucokinase activator clinical candidate for treating type 2 diabetes mellitus. J Med Chem 2012;55:1318-33.

8. Lloyd DJ, St Jean DJ, Jr., Kurzeja RJ, et al. Antidiabetic effects of glucokinase regulatory protein small-molecule disruptors. Nature 2013;504:437-40.

9. Brouwers MC, Jacobs C, Bast A, Stehouwer CD, Schaper NC. Modulation of Glucokinase Regulatory Protein: A Double-Edged Sword? Trends Mol Med 2015;21:583-94.

10. Beer NL, Tribble ND, McCulloch LJ, et al. The P446L variant in GCKR associated with fasting plasma glucose and triglyceride levels exerts its effect through increased glucokinase activity in liver. Hum Mol Genet 2009;18:4081-8.

11. Agius L. Glucokinase and molecular aspects of liver glycogen metabolism. Biochem J 2008;414:1-18.

12. Liu S, Ammirati MJ, Song $X$, et al. Insights into mechanism of glucokinase activation: observation of multiple distinct protein conformations. J Biol Chem 2012;287:13598-610.

13. Haeusler RA, Camastra S, Astiarraga B, Nannipieri M, Anselmino M, Ferrannini E. Decreased expression of hepatic glucokinase in type 2 diabetes. Mol Metab 2015;4:222-6.

14. Mooy JM, Grootenhuis PA, de Vries $\mathrm{H}$, et al. Prevalence and determinants of glucose intolerance in a Dutch caucasian population. The Hoorn Study. Diabetes Care 1995;18: 1270-3.

15. Henry RM, Kostense PJ, Spijkerman AM, et al. Arterial stiffness increases with deteriorating glucose tolerance status: the Hoorn Study. Circulation 2003;107:2089-95.

16. Spijkerman AM, Adriaanse MC, Dekker JM, et al. Diabetic patients detected by populationbased stepwise screening already have a diabetic cardiovascular risk profile. Diabetes Care 2002;25:1784-9.

17. Kershaw KN, Droomers M, Robinson WR, Carnethon MR, Daviglus ML, Monique Verschuren WM. Quantifying the contributions of behavioral and biological risk factors to socioeconomic disparities in coronary heart disease incidence: the MORGEN study. Eur J Epidemiol 2013;28:807-14.

18. van den Berg SW, Jansen EH, Kruijshoop M, et al. Paraoxonase 1 phenotype distribution and activity differs in subjects with newly diagnosed Type 2 diabetes (the CODAM Study). Diabet Med 2008;25:186-93.

19. Kruijshoop M, Feskens EJ, Blaak EE, de Bruin TW. Validation of capillary glucose measurements to detect glucose intolerance or type 2 diabetes mellitus in the general population. Clin Chim Acta 2004;341:33-40. 
20. van Moorsel D, van Greevenbroek MM, Schaper NC, et al. Bcll glucocorticoid receptor polymorphism in relation to cardiovascular variables: the Hoorn and CODAM studies. Eur $\mathrm{J}$ Endocrinol 2015;173:455-64.

21. Engelen L, Ferreira I, Gaens KH, et al. The association between the -374T/A polymorphism of the receptor for advanced glycation endproducts gene and blood pressure and arterial stiffness is modified by glucose metabolism status: the Hoorn and CoDAM studies. J Hypertens 2010;28:285-93.

22. Geelen CC, van Greevenbroek MM, van Rossum EF, et al. Bcll glucocorticoid receptor polymorphism is associated with greater body fatness: the Hoorn and CODAM studies. J Clin Endocrinol Metab 2013;98:E595-9.

23. Engelen L, Ferreira I, Brouwers $O$, et al. Polymorphisms in glyoxalase 1 gene are not associated with vascular complications: the Hoorn and CoDAM studies. J Hypertens 2009;27:1399-403.

24. van Woudenbergh GJ, Theofylaktopoulou D, Kuijsten A, et al. Adapted dietary inflammatory index and its association with a summary score for low-grade inflammation and markers of glucose metabolism: the Cohort study on Diabetes and Atherosclerosis Maastricht (CODAM) and the Hoorn study. Am J Clin Nutr 2013;98:1533-42.

25. Jacobs $M$, van Greevenbroek MM, van der Kallen CJ, et al. Low-grade inflammation can partly explain the association between the metabolic syndrome and either coronary artery disease or severity of peripheral arterial disease: the CODAM study. Eur J Clin Invest 2009;39:437-44.

26. WHO. Definition, diagnosis and classification of diabetes mellitus and its complications. Report of a WHO consultation. Part 1: diagnosis and classification of diabetes mellitus. Geneva1999.

27. Orho-Melander $M$, Melander $O$, Guiducci $C$, et al. Common missense variant in the glucokinase regulatory protein gene is associated with increased plasma triglyceride and Creactive protein but lower fasting glucose concentrations. Diabetes 2008;57:3112-21.

28. Vaxillaire M, Cavalcanti-Proenca C, Dechaume A, et al. The common P446L polymorphism in GCKR inversely modulates fasting glucose and triglyceride levels and reduces type 2 diabetes risk in the DESIR prospective general French population. Diabetes 2008;57:2253-7.

29. Taskinen MR. Diabetic dyslipidaemia: from basic research to clinical practice. Diabetologia 2003;46:733-49.

30. Lindholm E, Agardh E, Tuomi T, Groop L, Agardh CD. Classifying diabetes according to the new WHO clinical stages. Eur J Epidemiol 2001;17:983-9.

31. Cole CB, Nikpay M, Lau P, et al. Adiposity significantly modifies genetic risk for dyslipidemia. J Lipid Res 2014;55:2416-22.

32. Adiels M, Boren J, Caslake MJ, et al. Overproduction of VLDL1 driven by hyperglycemia is a dominant feature of diabetic dyslipidemia. Arterioscler Thromb Vasc Biol 2005;25:1697-703.

33. Vedala A, Wang W, Neese RA, Christiansen MP, Hellerstein MK. Delayed secretory pathway contributions to VLDL-triglycerides from plasma NEFA, diet, and de novo lipogenesis in humans. J Lipid Res 2006;47:2562-74.

34. Santoro N, Caprio S, Pierpont B, Van Name M, Savoye M, Parks EJ. Hepatic De Novo Lipogenesis in Obese Youth Is Modulated by a Common Variant in the GCKR Gene. J Clin Endocrinol Metab 2015;100:E1125-32.

35. Manolio TA, Collins FS, Cox NJ, et al. Finding the missing heritability of complex diseases. Nature 2009;461:747-53.

36. Ginsberg HN, Elam MB, Lovato LC, et al. Effects of combination lipid therapy in type 2 diabetes mellitus. N Engl J Med 2010;362:1563-74.

37. Cohen JC, Boerwinkle E, Mosley TH, Jr., Hobbs HH. Sequence variations in PCSK9, low LDL, and protection against coronary heart disease. N Engl J Med 2006;354:1264-72.

38. Robinson JG, Farnier M, Krempf M, et al. Efficacy and safety of alirocumab in reducing lipids and cardiovascular events. N Engl J Med 2015;372:1489-99. 



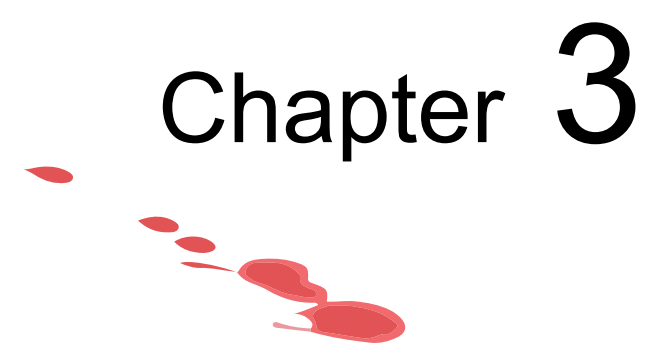

Effects of genetic variants in GCKR on coronary artery disease, glomerular filtration rate and chronic kidney disease

Association of common gene variants in glucokinase regulatory protein with cardiorenal disease: a systematic review and meta-analysis

Pomme 1.A.G. Simons*, Nynke Simons*, Coen D.A. Stehouwer, Casper G. Schalkwijk, Nicolaas C. Schaper, Martijn C.G.J. Brouwers*

PLoS One. 2018;13(10):e0206174 


\section{Abstract}

\section{Background}

Small-molecules that disrupt the binding between glucokinase and glucokinase regulatory protein in the liver represent a potential new class of glucoselowering drugs. It will, however, take years before their effects on clinically relevant cardiovascular endpoints are known. The purpose of this study was to estimate the effects of these drugs on cardiorenal outcomes by studying variants in the glucokinase regulatory protein gene (GCKR) that mimic glucokinase-glucokinase regulatory protein disruptors.

\section{Methods}

The MEDLINE and EMBASE databases were searched for studies reporting on the association between GCKR variants (rs1260326, rs780094 and rs780093) and coronary artery disease (CAD), estimated glomerular filtration rate (eGFR) and chronic kidney disease (CKD).

\section{Results}

In total 5 CAD studies ( $n=274,625$ individuals), 7 eGFR studies ( $n=195,195$ individuals) and 4 CKD studies ( $n=31,642$ cases and $n=408,432$ controls) were included. Meta-analysis revealed a significant association between GCKR variants and CAD (OR:1.02 per risk allele, 95\%Cl:1.00;1.04, $p=0.01$ ). Sensitivity analyses showed that replacement of one large, influential CAD study by two other, partly overlapping studies resulted in similar point estimates, albeit less precise (OR:1.02; 95\% Cl:0.98;1.06 and OR:1.02; 95\%Cl: $0.99 ; 1.04)$. GCKR was associated with an improved eGFR (+0.49 $\mathrm{ml} / \mathrm{min}$, $95 \% \mathrm{Cl}: 0.10 ; 0.89, \mathrm{p}=0.01)$ and a trend towards protection from CKD (OR:0.98, 95\%Cl:0.95;1.01, $p=0.13)$.

\section{Conclusion}

This study suggests that increased glucokinase-glucokinase regulatory protein disruption has beneficial effects on eGFR, but these may be offset by a disadvantageous effect on coronary artery disease risk. Further studies are warranted to elucidate the mechanistic link between hepatic glucose metabolism and eGFR. 


\section{Background}

In the current area of precise medicine, there is an ongoing search for new anti-diabetic medication with different modes of action. Drugs that modulate the function of glucokinase have been the scope of diabetes research for more than a decade now. ${ }^{1-4}$ Glucokinase plays a pivotal role in regulating pancreatic insulin secretion and hepatic glucose uptake, owing to its unique enzymatic actions. ${ }^{5}$ It catalyzes the conversion of glucose to glucose-6-phosphate, the first step in glycolysis. ${ }^{1-4}$ To date, however, clinical trials with glucokinase activators in patients with type 2 diabetes have been disappointing, since the glucose-lowering effects were non-sustained and accompanied by an increased risk of hypoglycemia and hypertriglyceridemia. ${ }^{1}$ Hepatoselective glucokinase activators could theoretically bypass some of these side-effects, in particular the risk of hypoglycemia. ${ }^{6}$

An alternative way to increase hepatic glucokinase activity is to disrupt the binding between glucokinase and glucokinase regulatory protein. Glucokinase regulatory protein is a liver-specific protein located in the nucleus that binds - and hence inactivates - glucokinase in the fasting state. In the postprandial state, glucokinase dissociates from glucokinase regulatory protein and subsequently migrates towards the cytosolic space where it facilitates phosphorylation of glucose. ${ }^{7,8}$ Lloyd and colleagues previously demonstrated that small molecules that disrupt the glucokinase-glucokinase regulatory protein complex reduce plasma glucose levels without causing hypoglycemia in mice. ${ }^{9}$ Although promising, it will probably take years before this new drug can be tested in a clinical setting.

Genetic epidemiology can be helpful to gain more insight into the clinical effects of glucokinase-glucokinase regulatory protein disruption in humans. Since individuals are 'randomized' at birth to receive a wildtype allele or a variant that encodes glucokinase regulatory protein that binds glucokinase less effectively, the effects of this variant on clinical endpoints can be studied as a surrogate for glucokinase-glucokinase regulatory protein disruptors. Such a Mendelian randomization approach has been proven to be effective in predicting the (un)intended effects of new drugs. ${ }^{10}$

We previously reviewed current literature on the cardiometabolic effects of variants in the glucokinase regulatory protein gene $(G C K R) .{ }^{11}$ Individuals carrying the variant that binds glucokinase less effectively are indeed characterized by reduced fasting plasma glucose levels, but this is accompanied by an increased risk of nonalcoholic fatty liver disease (NAFLD), 
hypertriglyceridemia and gout. ${ }^{12-14}$ Of interest, there are studies suggesting that the same variant protects from chronic kidney disease (CKD) ${ }^{15}$ Given these opposing effects it is difficult to predict what the net effect will be on coronary artery disease (CAD), one of the most clinically relevant outcomes in type 2 diabetes.

The aim of the present study was therefore to elucidate the association between GCKR and CAD and CKD by conducting a systematic review and meta-analysis.

\section{Methods}

Data sources, searches and study selection

The MEDLINE and EMBASE databases were searched for: 1) original, genetic association studies addressing the relationship between common variants in GCKR (rs1260326, rs780094 or rs780093) and CAD; and 2) genome-wide association studies (GWAS) on CAD, as they are likely to include the variants of interest (see Supplementary Table S3.1 for search strategy and Supplementary Figure S3.1 for flowchart). CAD was defined as myocardial infarction (MI), significant stenosis (i.e., $\geq 50 \%$ ) in one or more main coronary arteries or coronary intervention (including coronary artery bypass grafting $(C A B G)$ and percutaneous coronary intervention $(\mathrm{PCI})$ ).

A second search was performed for the association between the common variants in GCKR and renal function. Studies reporting serum creatinine levels, estimated glomerular filtration (eGFR) (based on serum creatinine or cystatin C), or presence of CKD were considered eligible (see Supplementary Table S3.2 for search strategy and Supplementary Figure S3.4 for flowchart).

Cross-sectional articles, written in English, German or Dutch, were included. No publication date or publication status restrictions were imposed. The electronic searches were conducted by one researcher (P.I.H.G.S.) and completed on March 6, 2018.

\section{Meta-analyses}

Two separate systematic reviews and three meta-analyses were conducted to determine the association between 1) common variants in GCKR and CAD; and 2) common variants in GCKR and renal function, i.e., eGFR and CKD 
(based on dichotomized eGFR). Selection of variants was primarily based on functionality, i.e., the variant has been shown to be functional and mimics the effects of glucokinase-glucokinase regulatory protein disruptors (i.e., rs $1260326^{16,17}$ ). In addition, variants that are in strong linkage disequilibrium with this functional variant, i.e., rs780094 or rs780093, were included as well $\left(r^{2} \approx 0.92\right.$ for both SNPs in both Europeans and East Asians; source: 1000 Genomes project phase 3 ). The systematic reviews and meta-analyses were performed according to the PRISMA statement with the only exception of a (registered) review protocol. ${ }^{18}$

\section{Data extraction and quality assessment}

Data extraction was done in a two-step, standardized fashion where one researcher (P.I.H.G.S.) extracted the data, which was subsequently checked by two other researchers (N.S. and M.C.G.J.B.). The following variables were extracted from the included studies: odds ratios or unstandardized beta coefficients, with $95 \%$ confidence intervals or standard errors. Authors were contacted in case of missing data (in particular for the GWAS). In case of nonresponse, a reminder was sent three weeks later. When more than one GCKR variant was reported, the functional variant (rs1260326) was chosen. The additive model was the preferred model of inheritance, based on previous GCKR association studies. ${ }^{19}$ Finally, given our interest in the systematic effects of GCKR per se, we aimed to obtain the crude outcome variables, i.e., without adjustment for potential mediators (e.g., plasma lipids levels).

To avoid inclusion of study cohorts that were reported more than once, in particular in GWAS consortia, special attention was paid to the origin of the individual study populations. In case of overlap, the study that contained the highest number of participants was included. The quality of the study and the risk of bias were assessed by two independent researchers (P.I.H.G.S. and N.S.) according to the Newcastle-Ottawa Scale (NOS). ${ }^{20}$

\section{Data synthesis and analysis}

Back-transformation of the log-transformed difference in eGFR between the two GCKR alleles was done as described elsewhere. ${ }^{21}$ Odds ratios and beta coefficients were meta-analyzed based on a random-effects model, using the DerSimonian-Laird method to incorporate between-study heterogeneity. Funnel plots were visually examined for asymmetry and analyzed by means of regression (Egger's test). 
Since most studies (in particular GWAS) only reported the principal summary measures (i.e., odds ratios) instead of individualized data, it was not feasible to adjust for potential environmental effects, nor was it possible to assess HardyWeinberg equilibrium or linkage disequilibrium for each study.

Sensitivity analyses were performed to assess the impact of studies that included subjects with different ancestries, studies with low quality (defined as a NOS score $<5$ stars) and studies that did not report crude (or age- and/or sex-adjusted) estimates. All analyses were conducted with the ' $R$ ' statistical software (R Developmental Core Team) using the metaphor package. ${ }^{22}$

\section{Results}

Systematic review and meta-analysis of the association between common variants in GCKR and CAD

The electronic search identified 3,051 unique records, which eventually resulted in five studies that were used for the meta-analysis ${ }^{23-27}$ (see Supplementary Figure S3.1 for flowchart and reasons for exclusion). All included studies were written in English. Twenty-six studies showed overlap with one of the included studies, i.e., the combined UK Biobank, CARDIoGRAMplusC4D 1000 genomes-based GWAS and Myocardial Infarction Genetics and CARDIoGRAM Exome datase ${ }^{24}$, and were therefore not included in the meta-analysis (Supplementary Table S3.3). The genetic variants of interest were often not reported in the main article, but could be found in the (online) supplementary materials. For one GWAS, the authors were contacted and the requested data were kindly provided. ${ }^{25}$

The characteristics of the included studies are shown in Table 3.1. In total, 274,625 subjects were included. In some, mainly Asian studies, the GCKR effect allele - defined as the allele that predisposes to reduced fasting plasma glucose levels (similar to the effect of a glucokinase-glucokinase regulatory protein disruptor) - was the predominant allele. The overall quality of the studies was good (Supplementary Table S3.4). 


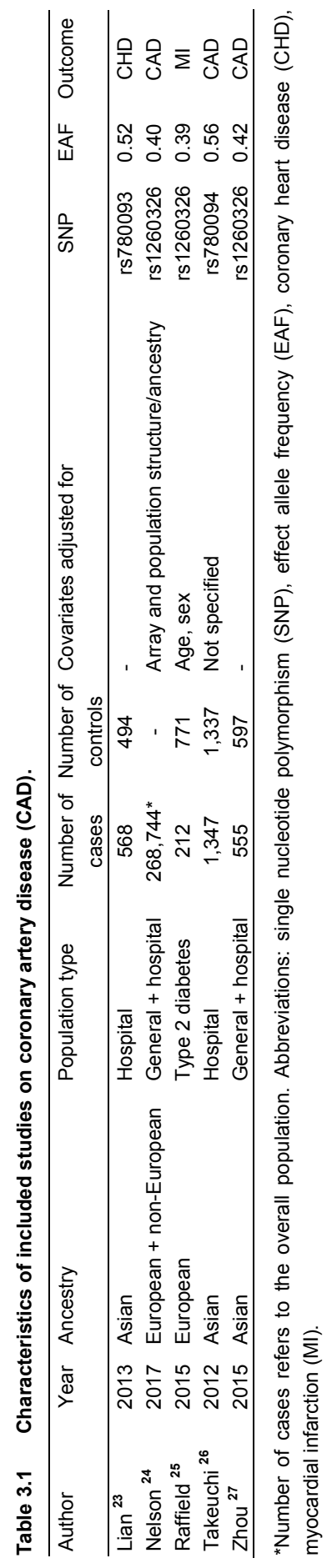


Meta-analysis demonstrated that the GCKR effect allele was significantly associated with CAD (OR: 1.02, 95\%Cl: 1.00;1.04, $\mathrm{p}=0.01$ ) (Figure 3.1). Heterogeneity was low $\left(Q=3.30, I^{2}=0 \%\right) .{ }^{28}$ Due to the low number of included studies, a funnel plot (or testing for funnel plot asymmetry) was not included, according to previous recommendations..$^{29,30}$ Since the meta-analysis was dominated by one large study, which is composed of 76 sub-studies $^{31}$, we conducted several sensitivity analyses to test the robustness of our findings. First, this large study was replaced by another large study that combined the CARDIoGRAMplusC4D 1000 genomes-based GWAS dataset with an additional 56,354 samples $(n=260,365$ subjects in total, Supplementary Table S3.3). ${ }^{32}$ The subsequent meta-analysis revealed a similar, but less precise point estimate (OR: $1.02,95 \% \mathrm{Cl}$ : 0.98;1.06, $\mathrm{p}=0.37$, Supplementary Figure $\mathrm{S} 3.2$ ).

The initial large study was also replaced by the CARDloGRAMplusC4D Metabochip data ${ }^{33,34}$, which overlaps for $\sim 55 \%$ with the CARDloGRAMplusC4D 1000 genomes-based GWAS data (Supplementary Table S3.3). ${ }^{35}$ This also allowed a better sensitivity analysis stratified by ancestry, since data for Europeans only have been presented separately. ${ }^{34}$ Again, the overall metaanalysis showed a similar, but non-significant point estimate (OR: $1.02,95 \% \mathrm{Cl}$ : $0.99 ; 1.04, p=0.27$, Supplementary Figure S3.3).

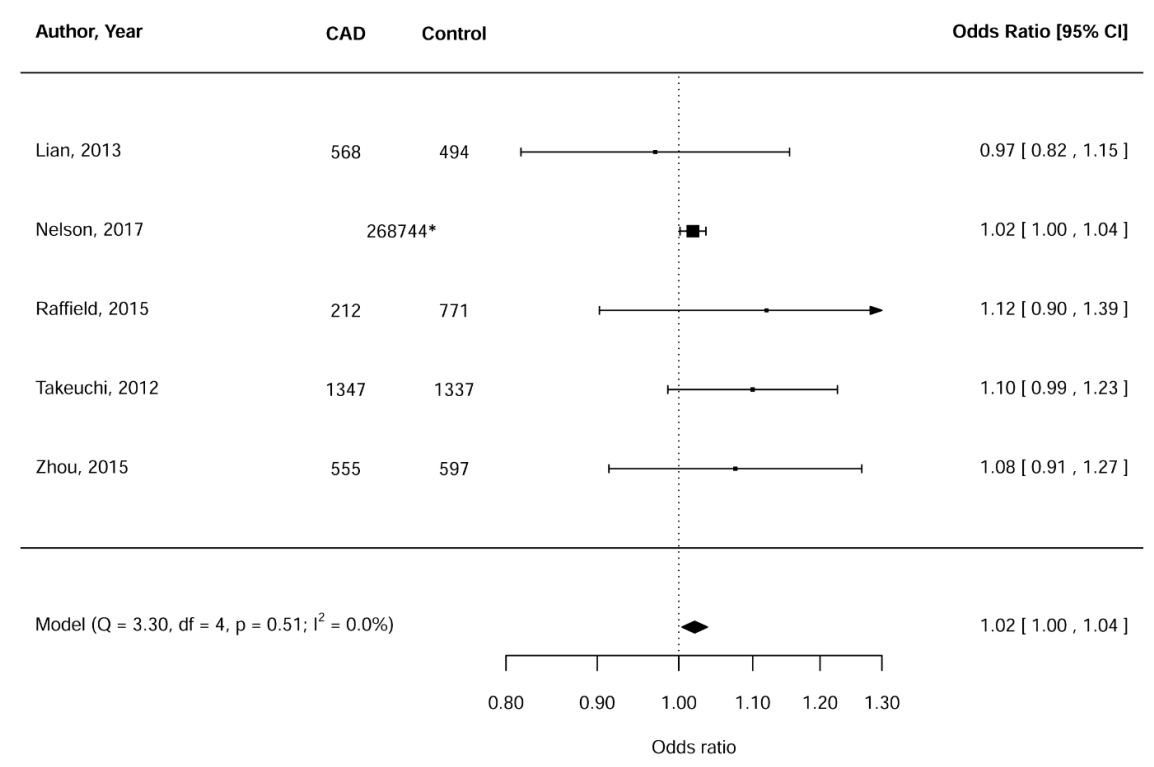

Figure 3.1 Meta-analysis of the relationship between the GCKR effect allele and coronary artery disease (CAD). *Number of individuals refers to the overall population. 
The GCKR effect allele was significantly associated with CAD in studies that included subjects of European ancestry only $(n=3)$ (OR: $1.02,95 \% \mathrm{Cl}$ : $1.00 ; 1.05, p=0.02)$, but not in studies that included subjects of Asian ancestry only $(n=3)(O R: 1.06,95 \% \mathrm{Cl}: 0.98 ; 1.15, p=0.13$; Supplementary Figure S3.3). Of note, these effect sizes were not statistically different $(p=0.36)$. Repeat analysis without the study with low quality ${ }^{25}$ (i.e., NOS score $<5$ stars) did not affect the primary outcome.

\section{Systematic review and meta-analysis of the association between common variants in GCKR and eGFR and CKD}

Of the 661 eligible records that were selected by our initial search, eight studies fulfilled all in- and exclusion criteria and were used for the meta-analyses (see Supplementary Figure S3.4 for flowchart and reasons for exclusion and Supplementary Table S3.5 for duplicate studies). All included studies were written in English. The genetic variants of interest were often not reported in the main article, but could be found in the (online) supplementary materials. For two GWAS, the authors were contacted and the requested data were kindly provided. ${ }^{36,37}$ Six studies reported data on creatinine-based eGFR ${ }^{36,38-42}$, one on cystatin C-based eGFR ${ }^{15}$ and four on CKD. ${ }^{36,37,40,42}$ Study characteristics of the eGFR and CKD studies are provided in Table 3.2. All studies used only the (creatinine-based) eGFR criterion to define CKD. Quality assessment of the included studies yielded an average score of five out of nine stars (Supplementary Table S3.6). Many studies reporting on eGFR scored low on 'comparability', i.e., the analyses were adjusted for covariates more than age and/or sex, whereas we aimed to obtain the crude relationship between GCKR and eGFR.

Meta-analysis, including 195,195 individuals, showed that the GCKR effect allele was significantly associated with an increased eGFR $(0.49 \mathrm{ml} / \mathrm{min}$, $95 \% \mathrm{Cl}: 0.10 ; 0.89, \mathrm{p}=0.01)$ (Figure 3.2). Heterogeneity was high $(\mathrm{Q}=43.12$, $\mathrm{I}^{2}=88.4 \%$ ). The only study that reported on cystatin C-based eGFR observed a similar effect size, which was statistically significant in the discovery cohort $(p=0.006)$, but not in the replication cohort $(p=0.16){ }^{15}$

The meta-analysis for CKD, including 31,642 cases and 408,432 controls, showed a protective effect of the GCKR effect allele on CKD, albeit not statistically significant (OR: $0.98,95 \% \mathrm{Cl}: 0.95 ; 1.01, p=0.13 ; \mathrm{Q}=5.54, \mathrm{I}^{2}=45.9 \%$ ) (Figure 3.3). The forest plot identified one outlying study that explained the moderate heterogeneity (Figure 3.3). Repeat analysis without this study ${ }^{40}$ resulted in a significant, negative relationship (OR: $0.97,95 \% \mathrm{Cl}: 0.95 ; 0.99$, $p=0.003$ ). The same study also accounted for the non-significant relationship 
with CKD when sensitivity analyses were conducted for Asian studies only (Supplementary Figure S3.5). All CKD studies were of sufficient quality (NOS score $\geq 5$ stars) and did not adjust for co-variates other than age and/or sex.

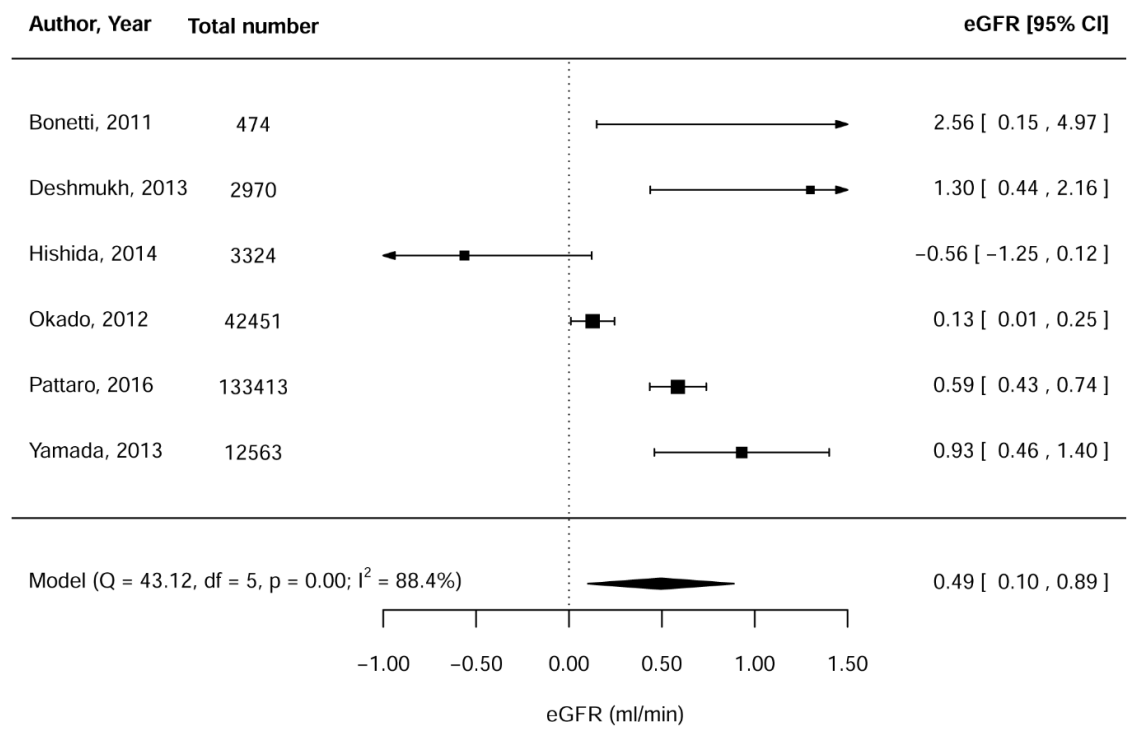

Figure 3.2 Meta-analysis of the relationship between the GCKR effect allele and creatinine-based estimated glomerular filtration rate (eGFR).

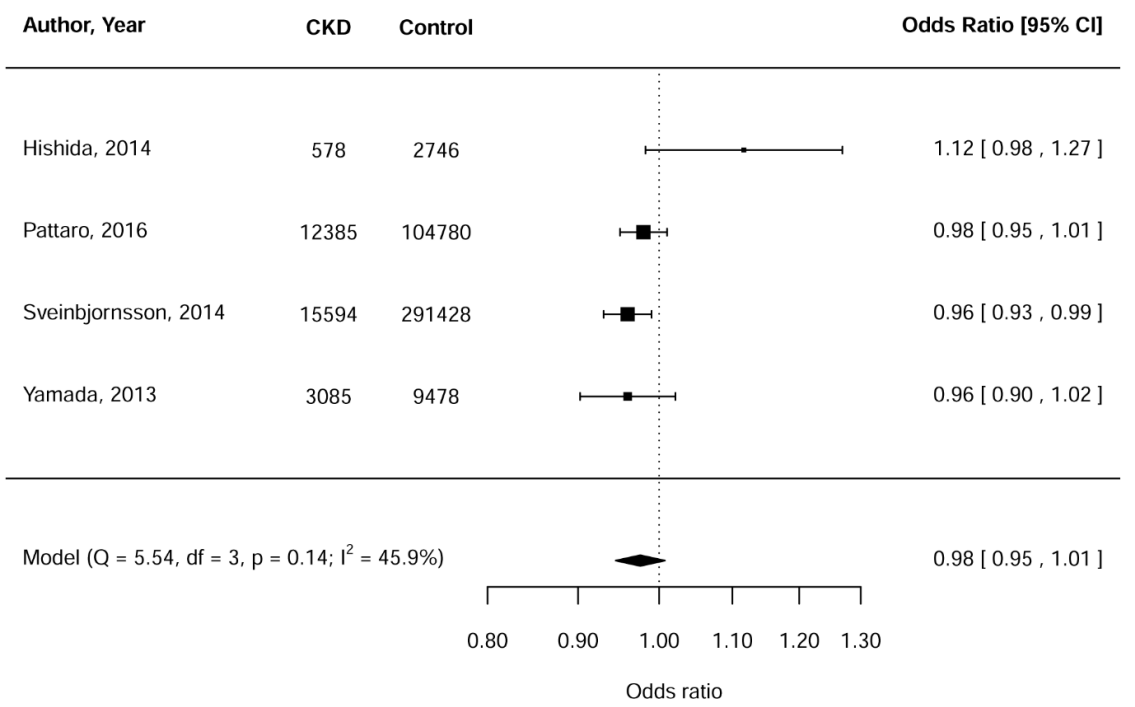

Figure 3.3 Meta-analysis of the relationship between the GCKR effect allele and chronic kidney disease (CKD). 


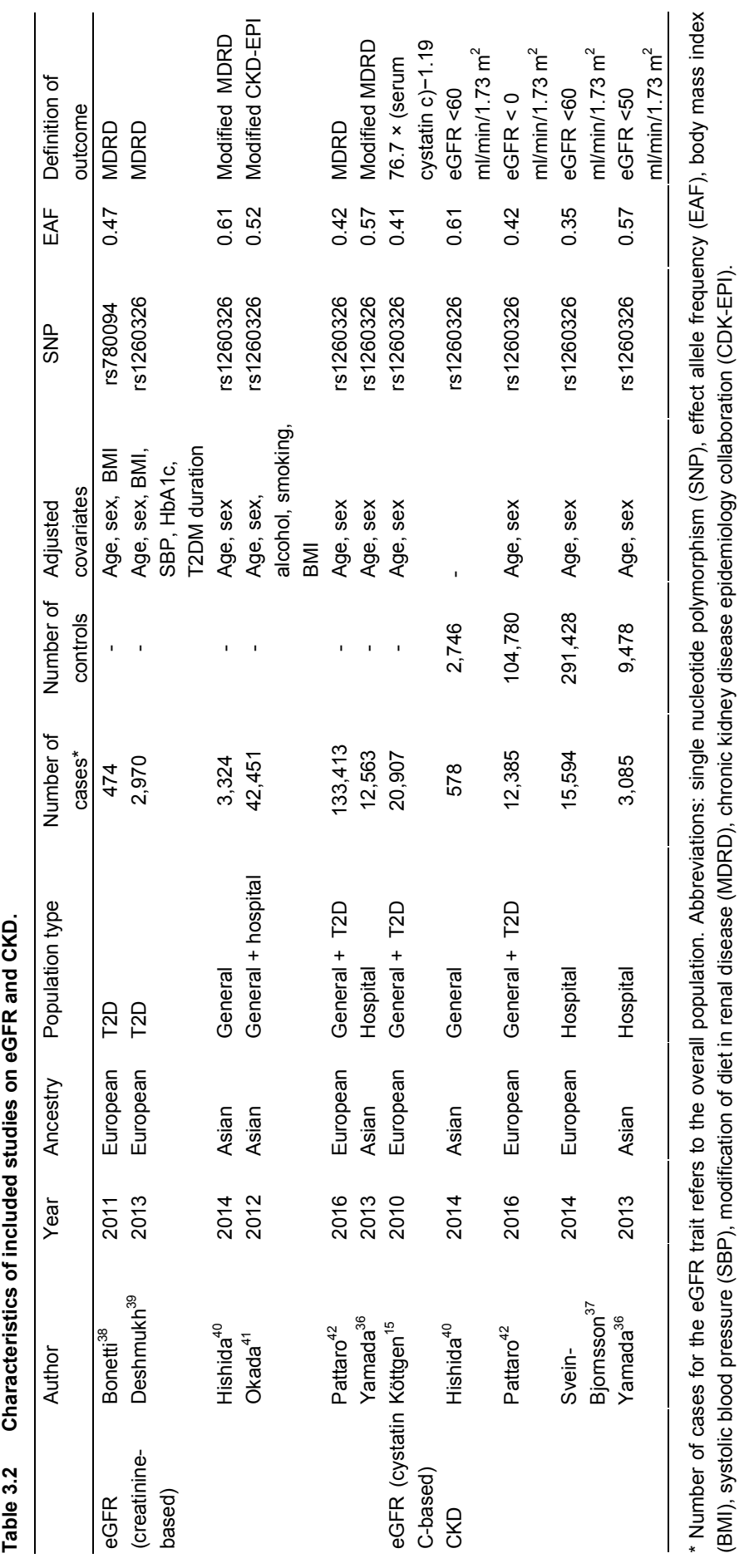




\section{Discussion}

Glucokinase regulatory protein is a liver-specific protein that plays an important role in the regulation of hepatic glucose uptake and, consequently, de novo lipogenesis, one of the principal pathways in the development of NAFLD. ${ }^{11}$ By studying the systemic effects of common variants in GCKR it is possible to gain more insight into the interaction between hepatic glucose metabolism and cardiorenal disease. Moreover, it allows an evaluation of small-molecule disruptors of the glucokinase-glucokinase regulatory protein complex as a potential new glucose-lowering treatment. In three meta-analyses using data from at least $\sim 200,000$ individuals, we showed that the GCKR effect allele which encodes a glucokinase regulatory protein that binds glucokinase less effectively - appeared to be associated with CAD, whereas a protective effect was observed for eGFR.

Previous studies have shown that the GCKR effect allele is associated with an atherogenic lipid profile, i.e., higher plasma triglycerides and apolipoprotein $B$ levels, reduced HDL cholesterol levels and the presence of small-dense LDL particles. ${ }^{12,43,44}$ In that respect it is of no surprise that we did observe a positive association of GCKR on CAD in our primary analysis. If, however, one would take into account the effect of GCKR on only plasma triglycerides, it would be anticipated to already result in an odds ratio of 1.05 to develop CAD. ${ }^{45}$ The smaller effect estimate that was found in this study (OR: $1.02,95 \% \mathrm{Cl}$ : $1.00 ; 1.04)$ should therefore be accounted for by another, protective factor that blunts the plasma lipid-mediated effects of GCKR on CAD risk. GCKR has previously been associated with reduced fasting plasma glucose levels. ${ }^{12}$ The hitherto reported protective effect of GCKR on eGFR could be another explanatory factor. Previous epidemiological studies have shown that CKD is an independent cardiovascular risk factor. ${ }^{46}$

The current meta-analyses were confined to creatinine-based renal outcome measures (eGFR and CKD), since these were most frequently reported. Köttgen and colleagues showed that the positive relationship between GCKR and (creatinine-based) eGFR was also observed for cystatin C-based eGFR. ${ }^{15}$ The same authors suggested that another gene, which is in linkage disequilibrium with GCKR, is actually responsible for the association with renal function. ${ }^{15}$ However, previous experiments in liver-specific glucokinase knockout mice - which are metabolically opposite to increased glucokinaseglucokinase regulatory protein disruption - are characterized by increased kidney damage ${ }^{47}$, which is in line with the current study. 
The mechanism by which enhanced glucokinase-glucokinase regulatory protein disruption exerts its renoprotective effects remains to be elucidated. The GCKR effect allele has been associated with increased NAFLD risk, low HDL cholesterol levels and higher urate levels ${ }^{12,13,43,44,48}$, which in turn have been associated with deterioration of renal function. ${ }^{49-51}$ These factors should therefore be outbalanced by factors that protect the kidney, such as lower plasma glucose levels. We cannot exclude that there are also other, yet unknown factors that contribute to the renoprotective effect of the GCKR effect allele. Further research is needed to identify these factors as it may have important clinical implications.

The present study may provide a glimpse into the future of what the cardiorenal effects of small-molecule disruptors of the glucokinase-glucokinase regulatory protein complex will be as a potential new glucose-lowering drug. Although the protective effect on eGFR and CKD appears to be promising at first sight, it may be outbalanced by an increased risk to develop CAD. Furthermore, a synergistic effect between GCKR and type 2 diabetes on CAD risk cannot be ruled out. We previously demonstrated that the effects of the GCKR effect allele on plasma lipid levels were more pronounced in patients with type 2 diabetes when compared to healthy individuals. ${ }^{52} \mathrm{~A}$ similar interaction between GCKR and type 2 diabetes on CAD risk would seriously decrease the applicability of small molecule disruptors of the glucokinase-glucokinase regulatory protein complex as new antidiabetic drug. Unfortunately, there were too few studies that were specifically conducted in type 2 diabetes to formally investigate such an interaction in the current meta-analysis.

This study has several strengths and limitations. First, the meta-analysis of the association of GCKR with CAD depends to a large extent on the the combined UK Biobank, CARDIoGRAMplusC4D 1000 genomes-based GWAS and Myocardial Infarction Genetics and CARDIoGRAM Exome dataset, which is actually a meta-analysis by itself. ${ }^{31}$ In subsequent sensitivity analyses we replaced this large dataset by other CARloGRAMplusC4D-based studies that despite a substantial overlap with the original study - included a large number of independent samples. ${ }^{32-34}$ Although similar effect sizes were observed, statistical significance was not reached. The positive association between the GCKR effect allele and CAD in the primary analysis should therefore be interpreted with some caution.

Second, the definition of CKD was only based on eGFR - not the presence of albuminuria - in all of the included studies. Both factors are part of the classification of CKD as defined by the Kidney Disease Improving Global Outcomes (KDIGO). ${ }^{53}$ The CKD Genetics Consortium recently reported that the 
GCKR variant that protects from deterioration of renal function is associated with an increased urine albumin-creatinine ratio. ${ }^{51}$ These findings emphasize the need for further research on the pathophysiological mechanisms relating glucokinase regulatory protein to the kidney.

Third, it is not entirely clear whether the effects of genetic variants in GCKR and small molecule disruptors of the glucokinase-glucokinase regulatory protein complex are truly comparable. This is one of the general limitations of the Mendelian randomization approach in which genetic variants are used as an instrument to study the effects of a specific drug of interest. However, previous experimental studies have shown that both the product of the GCKR minor allele and glucokinase-glucokinase regulatory protein disruptors cause an increased translocation of glucokinase from the nucleus towards the cytosolic space in the liver. ${ }^{9,17}$ This explains the reduced plasma glucose levels that have been associated with both the GCKR minor allele and treatment with glucokinase-glucokinase regulatory protein disruptors. ${ }^{9,54}$

Another aspect that deserves consideration is the moderate-to-high heterogeneity that was observed in some of the meta-analyses. This could be the result of genotyping errors or difference in methodology, such as discrepancies in outcome measures (particularly for CAD) or study populations (e.g., population-based versus hospital-based). Although ancestry did not account for the moderate-to-high heterogeneity, the number of studies was too small to make strong inferences. Furthermore, differences in diet could contribute to the observed heterogeneity given the previously reported GCKRdiet interaction on plasma triglycerides levels. ${ }^{55,56}$ It is, however, unlikely that these factors truly account for the opposing effect sizes that were present in the individual studies, e.g., GCKR seemed to protect from CKD in one Japanese cohort $^{36,37,41,42}$ whereas a predisposing effect appeared to be present in one other. ${ }^{40}$ These opposing effects could simply be the consequence of chance, especially in small-sized studies with few events. Alternatively, GCKR could theoretically be in linkage disequilibrium with a gene that exerts an opposing effect on cardiorenal risk in certain but not all populations. These opposing effects could have important therapeutic implications if they would be inherent to glucokinase regulatory protein function and therefore deserve further attention.

A final limitation was that we were forced to exclude a considerable amount of studies, and hence a substantial number of subjects, from the meta-analyses because of partial overlap of individual study cohorts. Yet, we were still able to include a high number of individuals, ranging from $\sim 200,000$ to 400,000 in the three meta-analyses, which can be attributed to our search strategy that was not confined to studies specifically reporting on GCKR. We correctly assumed 
that GWAS were likely to include our variants of interest without reporting in the manuscript's title or abstract.

In conclusion, the present study extends our knowledge on the systemic effects of enhanced disruption of the glucokinase-glucokinase regulatory protein complex by demonstrating that the GCKR effect allele is associated with a better eGFR. A disadvantageous effect on CAD risk can, however, not be ruled out. These findings question the benefits and applicability of small molecule disruptors of the glucokinase-glucokinase regulatory protein complex as a potential new class of antidiabetic drugs. Further studies are warranted to identify the factor that mediates the renoprotective effects of enhanced disruption of the glucokinase-glucokinase regulatory protein complex. 


\section{Literature}

1. Meininger GE, Scott R, Alba M, et al. Effects of MK-0941, a novel glucokinase activator, on glycemic control in insulin-treated patients with type 2 diabetes. Diabetes Care 2011;34: 2560-6.

2. Denney WS, Denham DS, Riggs MR, Amin NB. Glycemic Effect and Safety of a Systemic, Partial Glucokinase Activator, PF-04937319, in Patients With Type 2 Diabetes Mellitus Inadequately Controlled on Metformin-A Randomized, Crossover, Active-Controlled Study. Clinical pharmacology in drug development 2016;5:517-27.

3. Sarabu R, Bizzarro FT, Corbett WL, et al. Discovery of piragliatin--first glucokinase activator studied in type 2 diabetic patients. Journal of medicinal chemistry 2012;55:7021-36.

4. Johnson D, Shepherd RM, Gill D, Gorman T, Smith DM, Dunne MJ. Glucose-dependent modulation of insulin secretion and intracellular calcium ions by GKA50, a glucokinase activator. Diabetes 2007;56:1694-702.

5. Agius L. Glucokinase and molecular aspects of liver glycogen metabolism. Biochem J 2008;414:1-18.

6. Pfefferkorn JA, Guzman-Perez A, Litchfield J, et al. Discovery of (S)-6-(3-cyclopentyl-2-(4(trifluoromethyl)-1H-imidazol-1-yl)propanamido)nicotini $\mathrm{c}$ acid as a hepatoselective glucokinase activator clinical candidate for treating type 2 diabetes mellitus. Journal of medicinal chemistry 2012;55:1318-33.

7. de la Iglesia N, Veiga-da-Cunha M, Van Schaftingen E, Guinovart JJ, Ferrer JC. Glucokinase regulatory protein is essential for the proper subcellular localisation of liver glucokinase. FEBS letters 1999;456:332-8.

8. van Schaftingen $E$, Vandercammen $A$, Detheux $M$, Davies $D R$. The regulatory protein of liver glucokinase. Advances in enzyme regulation 1992;32:133-48.

9. Lloyd DJ, St Jean DJ, Jr., Kurzeja RJ, et al. Antidiabetic effects of glucokinase regulatory protein small-molecule disruptors. Nature 2013;504:437-40.

10. Walker VM, Davey Smith G, Davies NM, Martin RM. Mendelian randomization: a novel approach for the prediction of adverse drug events and drug repurposing opportunities. Int $\mathrm{J}$ Epidemiol 2017;46:2078-89.

11. Brouwers MC, Jacobs C, Bast A, Stehouwer CD, Schaper NC. Modulation of Glucokinase Regulatory Protein: A Double-Edged Sword? Trends Mol Med 2015;21:583-94.

12. Orho-Melander $\mathrm{M}$, Melander $\mathrm{O}$, Guiducci $\mathrm{C}$, et al. Common missense variant in the glucokinase regulatory protein gene is associated with increased plasma triglyceride and Creactive protein but lower fasting glucose concentrations. Diabetes 2008;57:3112-21.

13. Speliotes EK, Yerges-Armstrong LM, Wu J, et al. Genome-wide association analysis identifies variants associated with nonalcoholic fatty liver disease that have distinct effects on metabolic traits. PLoS genetics 2011;7:e1001324.

14. Matsuo $\mathrm{H}$, Yamamoto $\mathrm{K}$, Nakaoka $\mathrm{H}$, et al. Genome-wide association study of clinically defined gout identifies multiple risk loci and its association with clinical subtypes. Annals of the rheumatic diseases 2016;75:652-9.

15. Kottgen $A$, Pattaro $C$, Boger $C A$, et al. New loci associated with kidney function and chronic kidney disease. Nat Genet 2010;42:376-84.

16. Beer NL, Tribble ND, McCulloch LJ, et al. The P446L variant in GCKR associated with fasting plasma glucose and triglyceride levels exerts its effect through increased glucokinase activity in liver. Human molecular genetics 2009;18:4081-8.

17. Rees MG, Wincovitch S, Schultz J, et al. Cellular characterisation of the GCKR P446L variant associated with type 2 diabetes risk. Diabetologia 2012;55:114-22.

18. Moher D, Liberati A, Tetzlaff J, Altman DG. Preferred reporting items for systematic reviews and meta-analyses: the PRISMA statement. BMJ 2009;339:b2535. 
19. Sparso T, Andersen G, Nielsen T, et al. The GCKR rs780094 polymorphism is associated with elevated fasting serum triacylglycerol, reduced fasting and OGTT-related insulinaemia, and reduced risk of type 2 diabetes. Diabetologia 2008;51:70-5.

20. Wells GA, Shea B, O'Connell D, et al. The Newcastle-Ottawa Scale (NOS) for Assessing the Quality of Non-Randomized Studies in Meta-Analysis. 2010;50:1088-10.

21. Higgins JP, White IR, Anzures-Cabrera J. Meta-analysis of skewed data: combining results reported on log-transformed or raw scales. Stat Med 2008;27:6072-92.

22. Viechtbauer $\mathrm{W}$. Conducting meta-analyses in $\mathrm{R}$ with the metafor package. Journal of Statistical Software 2010;36:1-48.

23. Lian J, Guo J, Chen Z, et al. Positive association between GCKR rs780093 polymorphism and coronary heart disease in the aged Han Chinese. Dis Markers 2013;35:863-8.

24. Nelson CP, Goel A, Butterworth AS, et al. Association analyses based on false discovery rate implicate new loci for coronary artery disease. Nat Genet 2017;49:1385-91.

25. Raffield LM, Cox AJ, Carr JJ, et al. Analysis of a cardiovascular disease genetic risk score in the Diabetes Heart Study. Acta Diabetol 2015;52:743-51.

26. Takeuchi $F$, Isono $M$, Katsuya $T$, et al. Association of genetic variants influencing lipid levels with coronary artery disease in Japanese individuals. PLoS One 2012;7:e46385.

27. Zhou YJ, Hong SC, Yin RX, Yang Q, Cao XL, Chen WX. Polymorphisms in the GCKR are associated with serum lipid traits, the risk of coronary artery disease and ischemic stroke. Int J Clin Exp Med 2015;8:10678-86.

28. Higgins JP, Thompson SG, Deeks JJ, Altman DG. Measuring inconsistency in metaanalyses. BMJ 2003;327:557-60.

29. Sterne JA, Sutton AJ, loannidis JP, et al. Recommendations for examining and interpreting funnel plot asymmetry in meta-analyses of randomised controlled trials. BMJ 2011;343:d4002.

30. Higgins JPT, Green S, (editors). Cochrane Handbook for Systematic Reviews of Interventions Version 5.1.0 [updated March 2011]. The Cochrane Collaboration, 2011. Available from http://handbook.cochrane.org.

31. Nelson CP, Goel A, Butterworth AS, et al. Association analyses based on false discovery rate implicate new loci for coronary artery disease. Nat Genet 2017;49:1385-91.

32. Zhao W, Rasheed A, Tikkanen E, et al. Identification of new susceptibility loci for type 2 diabetes and shared etiological pathways with coronary heart disease. Nat Genet 2017;49:1450-7.

33. Deloukas $\mathrm{P}$, Kanoni $\mathrm{S}$, Willenborg $\mathrm{C}$, et al. Large-scale association analysis identifies new risk loci for coronary artery disease. Nature Genetics 2013;45:25-33.

34. Schunkert H, Konig IR, Kathiresan S, et al. Large-scale association analysis identifies 13 new susceptibility loci for coronary artery disease. Nat Genet 2011;43:333-8.

35. Nikpay M, Goel A, Won HH, et al. A comprehensive 1,000 Genomes-based genome-wide association meta-analysis of coronary artery disease. Nat Genet 2015;47:1121-30.

36. Yamada $\mathrm{Y}$, Nishida $\mathrm{T}$, Ichihara $\mathrm{S}$, et al. Identification of chromosome $3 \mathrm{q} 28$ and ALPK1 as susceptibility loci for chronic kidney disease in Japanese individuals by a genome-wide association study. J Med Genet 2013;50:410-8.

37. Sveinbjornsson G, Mikaelsdottir E, Palsson R, et al. Rare mutations associating with serum creatinine and chronic kidney disease. Hum Mol Genet 2014;23:6935-43.

38. Bonetti S, Trombetta M, Boselli ML, et al. Variants of GCKR affect both beta-cell and kidney function in patients with newly diagnosed type 2 diabetes: the Verona newly diagnosed type 2 diabetes study 2. Diabetes Care 2011;34:1205-10.

39. Deshmukh HA, Palmer CN, Morris AD, Colhoun HM. Investigation of known estimated glomerular filtration rate loci in patients with type 2 diabetes. Diabet Med 2013;30:1230-5.

40. Hishida A, Takashima N, Turin TC, et al. GCK, GCKR polymorphisms and risk of chronic kidney disease in Japanese individuals: data from the J-MICC Study. J Nephrol 2014;27: 143-9. 
41. Okada $Y, \operatorname{Sim} X$, Go MJ, et al. Meta-analysis identifies multiple loci associated with kidney function-related traits in east Asian populations. Nat Genet 2012;44:904-9.

42. Pattaro C, Teumer A, Gorski M, et al. Genetic associations at 53 loci highlight cell types and biological pathways relevant for kidney function. Nat Commun 2016;7:10023.

43. Chambers JC, Zhang W, Sehmi J, et al. Genome-wide association study identifies loci influencing concentrations of liver enzymes in plasma. Nat Genet 2011;43:1131-8.

44. Kozian DH, Barthel A, Cousin E, et al. Glucokinase-activating GCKR polymorphisms increase plasma levels of triglycerides and free fatty acids, but do not elevate cardiovascular risk in the Ludwigshafen Risk and Cardiovascular Health Study. Hormone and metabolic research $=$ Hormon- und Stoffwechselforschung $=$ Hormones et metabolisme 2010;42:502-6.

45. Do R, Willer CJ, Schmidt EM, et al. Common variants associated with plasma triglycerides and risk for coronary artery disease. Nat Genet 2013;45:1345-52.

46. Go AS, Chertow GM, Fan D, McCulloch CE, Hsu C-y. Chronic Kidney Disease and the Risks of Death, Cardiovascular Events, and Hospitalization. New England Journal of Medicine 2004;351:1296-305.

47. Gu Y, Mao Y, Li H, et al. Long-term renal changes in the liver-specific glucokinase knockout mouse: implications for renal disease in MODY2. Translational research : the journal of laboratory and clinical medicine 2011;157:111-6.

48. Kolz M, Johnson T, Sanna S, et al. Meta-analysis of 28,141 individuals identifies common variants within five new loci that influence uric acid concentrations. PLoS Genet 2009;5:e1000504.

49. Musso G, Gambino R, Tabibian JH, et al. Association of Non-alcoholic Fatty Liver Disease with Chronic Kidney Disease: A Systematic Review and Meta-analysis. PLOS Medicine 2014;11:e1001680.

50. De Cosmo S, Viazzi F, Pacilli A, et al. Serum Uric Acid and Risk of CKD in Type 2 Diabetes. Clinical journal of the American Society of Nephrology : CJASN 2015;10:1921-9.

51. Lanktree MB, Theriault S, Walsh M, Pare G. HDL Cholesterol, LDL Cholesterol, and Triglycerides as Risk Factors for CKD: A Mendelian Randomization Study. Am J Kidney Dis 2017.

52. Simons N, Dekker JM, van Greevenbroek MM, et al. A Common Gene Variant in Glucokinase Regulatory Protein Interacts With Glucose Metabolism on Diabetic Dyslipidemia: the Combined CODAM and Hoorn Studies. Diabetes Care 2016;39:1811-7.

53. Ketteler M, Block GA, Evenepoel P, et al. Executive summary of the 2017 KDIGO Chronic Kidney Disease-Mineral and Bone Disorder (CKD-MBD) Guideline Update: what's changed and why it matters. Kidney Int 2017;92:26-36.

54. Dupuis J, Langenberg C, Prokopenko I, et al. New genetic loci implicated in fasting glucose homeostasis and their impact on type 2 diabetes risk. Nat Genet 2010;42:105-16.

55. Tam CH, Wang $\mathrm{Y}$, Lee HM, et al. Early gene-diet interaction between glucokinase regulatory protein (GCKR) polymorphism, vegetable and fish intakes in modulating triglyceride levels in healthy adolescents. Nutr Metab Cardiovasc Dis 2015;25:951-8.

56. Rousseaux J, Duhamel A, Dumont $\mathrm{J}$, et al. The $\mathrm{n}-3$ long-chain PUFAs modulate the impact of the GCKR Pro446Leu polymorphism on triglycerides in adolescents. J Lipid Res 2015;56: $1774-80$. 


\section{Supplementary Materials}

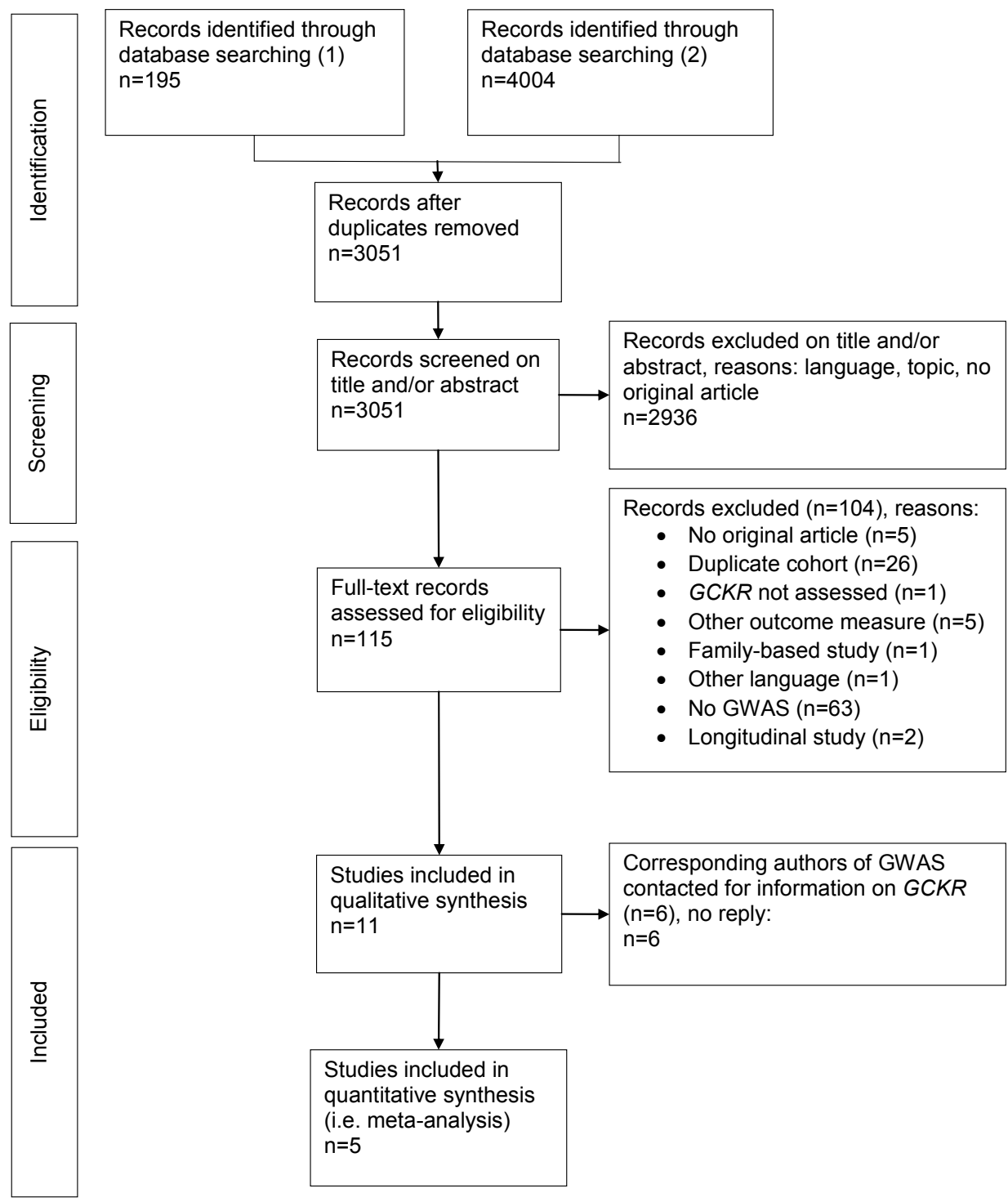

Figure S3.1 Flowchart of the systematic review on coronary artery disease (CAD). 


\begin{tabular}{|c|c|c|c|c|c|c|c|}
\hline Author, Year & CAD & Control & & & & \multicolumn{2}{|c|}{ Odds Ratio $[95 \% \mathrm{Cl}]$} \\
\hline Lian, 2013 & 568 & 494 & & & 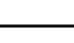 & & $0.97[0.82,1.15]$ \\
\hline Raffield, 2015 & 212 & 771 & & & & $\rightarrow$ & $1.12[0.90,1.39]$ \\
\hline Takeuchi, 2012 & 1347 & 1337 & & : & & & $1.10[0.99,1.23]$ \\
\hline Zhao, 2017 & 90381 & 169984 & & & & & $1.00[0.99,1.02]$ \\
\hline Zhou, 2015 & 555 & 597 & & & & & $1.08[0.91,1.27]$ \\
\hline \multicolumn{6}{|c|}{ Model $\left(Q=4.57, d f=4, p=0.33 ; I^{2}=12.5 \%\right)$} & & $1.02[0.98,1.06]$ \\
\hline & & $\Gamma$ & T & jं & T & 7 & \\
\hline & & 0.80 & 0.90 & 1.00 & 1.10 & 1.30 & \\
\hline
\end{tabular}

Figure S3.2 Forest plot of the meta-analysis on CAD - Sensitivity analysis. For this analysis, the combined UK Biobank, CARDIoGRAMplusC4D 1000 genomes-based GWAS and Myocardial Infarction Genetics and CARDIoGRAM Exome dataset ${ }^{1}$ was replaced by CARDIoGRAMplusC4D 1000 genomes-based GWAS dataset combined with 56,354 samples. $^{2}$ 


\section{European ancestry}

Raffield, 2015

Schunkert, 2011

Varbo, 2011

RE Model for Subgroup

\section{Asian ancestry}

Lian, 2013

Takeuchi, 2012

Zhou, 2015

RE Model for Subgroup

Combined European/Asian

Deloukas, 2013

RE Model for Subgroup

$1.12[0.90,1.39]$

$1.02[0.99,1.04]$

$1.03[1.00,1.06]$

$1.02[1.00,1.05]$

$0.97[0.82,1.15]$

$1.10[0.99,1.23$ ]

$1.08[0.91,1.27$ ]

1.06 [ $0.98,1.15]$

0.98 [ $0.96,1.00$ ]

$0.98[0.96,1.00]$

$1.02[0.99,1.05]$

RE Model for All Studies

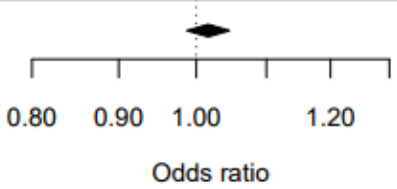

Figure S3.3 Forest plot of the meta-analysis on CAD - Stratified by ancestry. For this analysis, the combined UK Biobank, CARDloGRAMplusC4D 1000 genomes-based GWAS and Myocardial Infarction Genetics and CARDIoGRAM Exome dataset ${ }^{1}$ was replaced by the CARDIoGRAMplusC4D Metabochip dataset ${ }^{3,4}$, which allows stratification by ancestry. The CARDIoGRAMplusC4D Metabochip dataset overlaps for $\sim 55 \%$ with the CARDloGRAMplusC4D 1000 genomes-based GWAS. The Copenhagen City Heart Study, the Copenhagen General Population Study and the Copenhagen Ischemic Heart Disease Study ${ }^{5}$ were not part of the CARDIoGRAMplusC4D Metabochip dataset and were therefore included in this meta-analysis. 


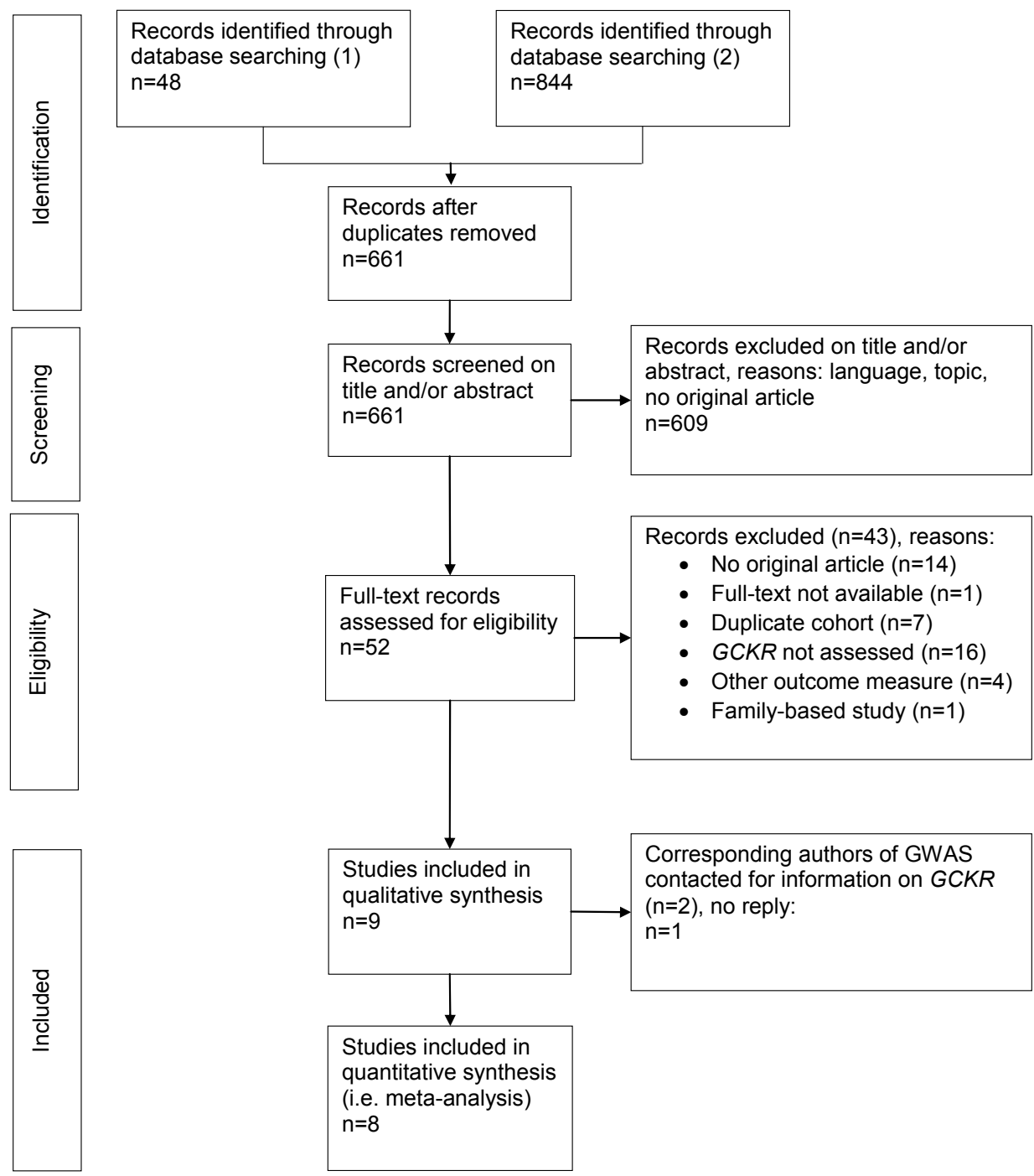

Figure S3.4 Flowchart of the systematic review on eGFR and CKD. 


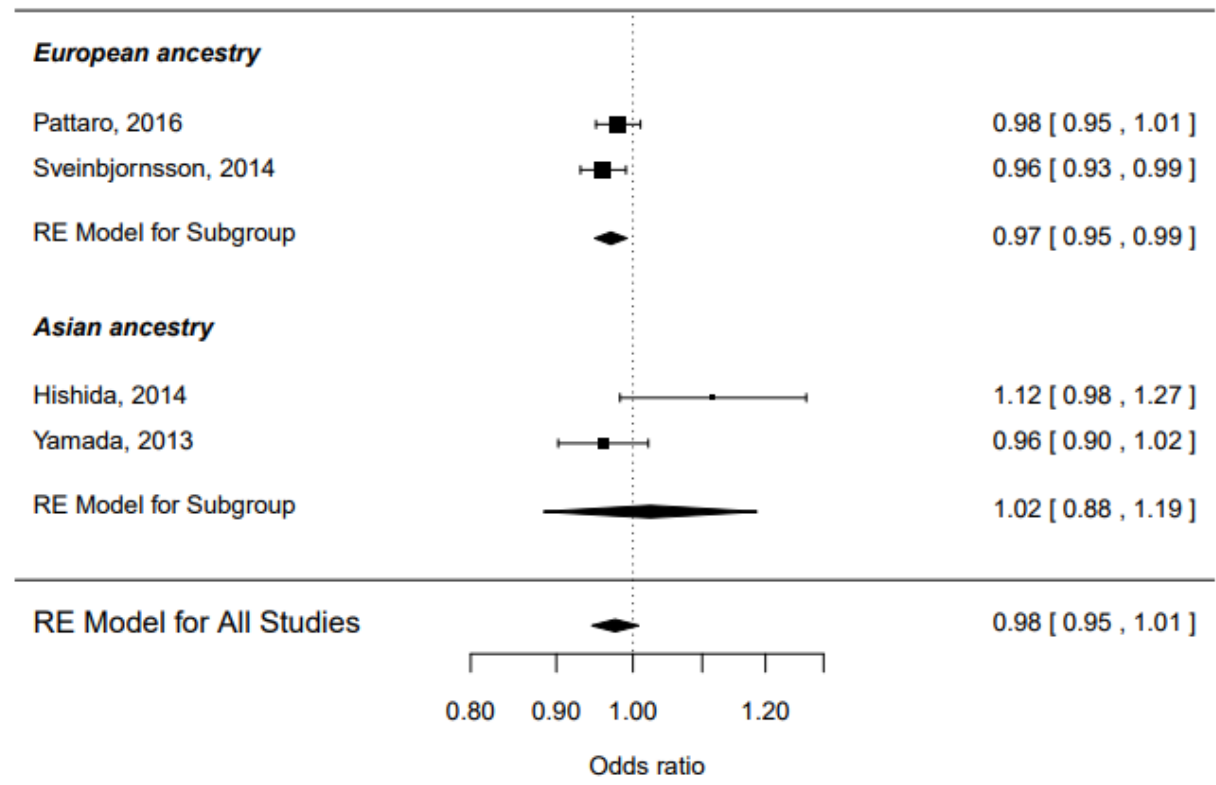

Figure S3.5 Forest plot of the meta-analysis on CKD - Stratified by ancestry. 
Table S3.1 Search strategy for CAD.

Search strategy: MEDLINE (OVID) 1946 to 2018 week 10, EMBASE (OVID) 1974 to 2018 week 10.

Search strategy 1: CAD and GCKR

1. Acute coronary syndrome/

2. Coronary artery disease/

3. Ischemic heart disease/

4. Heart disease/

5. Coronary artery atherosclerosis/

6. Coronary artery occlusion/

7. Coronary artery thrombosis/

8. Cardiovascular disease/

9. Myocardial infarction/

10. Stroke/

11. Cerebrovascular accident/

12. Peripheral vascular disease/

13. Transient ischemic attack/

14. Peripheral arterial disease/

15. Acute coronary syndrome.mp

16. Coronary artery disease. $\mathrm{mp}$

17. Ischemic heart disease. $\mathrm{mp}$

18. Heart disease.mp

19. Coronary artery atherosclerosis.mp

20. Coronary artery occlusion.mp

21. Coronary artery thrombosis.mp

22. CAD.mp

23. Cardiovascular disease.mp

24. CVD.mp

25. Cardiac attack.mp

26. Heart attack.mp

27. Myocardial infarction.mp

28. MI.mp

29. Stroke.mp

30. Cerebrovascular accident.mp

31. Ischemic stroke.mp

32. Peripheral vascular disease.mp

33. Transient ischemic attack.mp

34. TIA.mp

35. Peripheral artery disease.mp

36. CVA.mp

37. GCKR.mp

38. GKRP.mp

39. Glucokinase regulatory protein.mp

40. Rs1260326.mp

41. P446L.mp

42. Rs780094.mp

43. Rs780093.mp

44. 1 or 2 or 3 or 4 or 5 or 6 or 7 or 8 or 9 or 10 or 11 or 12 or 13 or 14 or 15 or 16 or 17 or 18 or 19 or 20 or 21 or 22 or 23 or 24 or 25 or 26 or 27 or 28 or 29 or 30 or 31 or 32 or 33 or 34 or 35 or 36

45. 37 or 38 or 39 or 40 or 41 or 42 or 43

46. 44 and 45

MEDLINE $=82$ retrieved, $E M B A S E=113$ retrieved 


\section{Search strategy 2: CAD and GWAS}

1. Acute coronary syndrome/

2. Coronary artery disease/

3. Ischemic heart disease/

4. Heart disease/

5. Coronary artery atherosclerosis/

6. Coronary artery occlusion/

7. Coronary artery thrombosis/

8. Cardiovascular disease/

9. Myocardial infarction/

10. Stroke/

11. Cerebrovascular accident/

12. Peripheral vascular disease/

13. Transient ischemic attack/

14. Peripheral arterial disease/

15. Acute coronary syndrome.mp

16. Coronary artery disease.mp

17. Ischemic heart disease.mp

18. Heart disease.mp

19. Coronary artery atherosclerosis.mp

20. Coronary artery occlusion.mp

21. Coronary artery thrombosis.mp

22. CAD.mp

23. Cardiovascular disease.mp

24. CVD.mp

25. Cardiac attack.mp

26. Heart attack.mp

27. Myocardial infarction.mp

28. MI.mp

29. Stroke.mp

30. Cerebrovascular accident.mp

31. Ischemic stroke.mp

32. Peripheral vascular disease.mp

33. Transient ischemic attack.mp

34. TIA.mp

35. Peripheral artery disease.mp

36. CVA.mp

37. GWAS.mp

38. GWA study.mp

39. Genome-wide association study/

40. Genome wide association study.mp

41. Whole genome association study.mp

42. WGA study.mp

43. WGAS.mp

44. 1 or 2 or 3 or 4 or 5 or 6 or 7 or 8 or 9 or 10 or 11 or 12 or 13 or 14 or 15 or 16 or 17 or 18 or 19 or 20 or 21 or 22 or 23 or 24 or 25 or 26 or 27 or 28 or 29 or 30 or 31 or 32 or 33 or 34 or 35 or 36

45. 37 or 38 or 39 or 40 or 41 or 42 or 43

46. 43 and 45

MEDLINE $=2026$ retrieved, EMBASE $=1978$ retrieved 
Table S3.2 Search strategy for eGFR and CKD.

Search strategy: MEDLINE (OVID) 1946 to 2018 week 10, EMBASE (OVID) 1974 to 2018 week 10.

Search strategy 1: CKD and GCKR

1. Kidney disease/

2. Renal insufficiency, chronic/

3. Kidney failure, chronic/

4. Glomerular filtration rate/

5. Creatine/

6. Cystatin $\mathrm{C} /$

7. Chronic kidney disease/

8. Glomerulopathy/

9. Kidney disease.mp

10. Chronic renal insufficiency.mp

11. Chronic kidney failure. $\mathrm{mp}$

12. Glomerular filtration rate. $\mathrm{mp}$

13. Creatine.mp

14. Cystatin C.mp

15. Chronic kidney disease.mp

16. CKD.mp

17. Renal disease.mp

18. Glomerulopathy.mp

19. GFR.mp

20. Estimated glomerular filtration rate.mp

21. eGFR.mp

22. GCKR.mp

23. GKRP.mp

24. Glucokinase regulatory protein.mp

25. Rs1260326.mp

26. P446L.mp

27. Rs780094.mp

28. Rs780093.mp

29. 1 or 2 or 3 or 4 or 5 or 6 or 7 or 8 or 9 or 10 or 11 or 12 or 13 or 14 or 15 or 16 or 17 or 18 or 19 or 20 or 21

30. 22 or 23 or 24 or 25 or 26 or 27 or 28

31. 29 and 30

$M E D L I N E=20$ retrieved, $E M B A S E=28$ retrieved 


\section{Search strategy 2: CKD and GWAS}

1. Kidney disease/

2. Renal insufficiency, chronic/

3. Kidney failure, chronic/

4. Glomerular filtration rate/

5. Creatine/

6. Cystatin $\mathrm{Cl}$

7. Chronic kidney disease/

8. Glomerulopathy/

9. Kidney disease.mp

10. Chronic renal insufficiency.mp

11. Chronic kidney failure.mp

12. Glomerular filtration rate. $\mathrm{mp}$

13. Creatine.mp

14. Cystatin C.mp

15. Chronic kidney disease.mp

16. CKD.mp

17. Renal disease.mp

18. Glomerulopathy.mp

19. GFR.mp

20. Estimated glomerular filtration rate. $\mathrm{mp}$

21. eGFR.mp

22. GWAS.mp

23. GWA study.mp

24. Genome-wide association study/

25. Genome wide association study.mp

26. Whole genome association study.mp

27. WGA study.mp

28. WGAS.mp

29. 1 or 2 or 3 or 4 or 5 or 6 or 7 or 8 or 9 or 10 or 11 or 12 or 13 or 14 or 15 or 16 or 17 or 18 or 19 or 20 or 21

30. 22 or 23 or 24 or 25 or 26 or 27 or 28

31. 29 and 30

MEDLINE $=352$ retrieved, EMBASE $=492$ retrieved 
Table S3.3 Overview of the excluded CAD studies with duplicate cohorts.

\begin{tabular}{|c|c|c|}
\hline Excluded study & Study cohorts (with duplicate cohort in bold) & $\begin{array}{l}\text { Study in which } \\
\text { duplicate cohort is } \\
\text { included }\end{array}$ \\
\hline Angelakopoulou $(2012)^{6}$ & $\begin{array}{l}\text { Northwick Park Heart Study II (NPHS II), British Regional } \\
\text { Heart Study (BRHS), English Longitudinal Study of Ageing } \\
\text { (ELSA), Edinburgh Artery Study (EAS), Whitehall II study, } \\
\text { The } 1958 \text { Birth Cohort (1958BC), The Medical Research } \\
\text { Council National Survey of Health and Development } \\
\text { (NSHD), Southampton Atherosclerosis Study (SAS), The } \\
\text { Stockholm Heart Epidemiology Program (SHEEP), The } \\
\text { Wellcome Trust Case Control Consortium (WTCCC), } \\
\text { University College Diabetes and Cardiovascular Study } \\
\text { (UDACS), Ealing Diabetes Study of Coagulation (EDS), The } \\
\text { MRC British Genetics of Hypertension (BRIGHT) }\end{array}$ & Nelson $(2017)^{1}$ \\
\hline $\mathrm{Bi}(2010)^{7}$ & The atherosclerosis risk in communities (ARIC) ${ }^{*}$ & Nelson $(2017)^{1}$ \\
\hline Burton $(2007)^{8}$ & WTCCC, 1958 British Birth Cohort, UK blood donor service & Nelson $(2017)^{1}$ \\
\hline Davies $(2012)^{9}$ & $\begin{array}{l}\text { The Ottowa heart genomics study (OHGS), Cleveland } \\
\text { clinic gene bank (CCGB), WTCCC, INTERHEART, Duke } \\
\text { Cathgen Study (DUKE) }\end{array}$ & Nelson $(2017)^{1}$ \\
\hline Dehghan $(2016)^{10}$ & $\begin{array}{l}\text { Age, gene/environment susceptibility-Reykjavik Study } \\
\text { (AGES)*, ARIC*, cardiovascular health study (CHS), } \\
\text { Family heart study (FHS), Rotterdam study }\end{array}$ & Nelson $(2017)^{1}$ \\
\hline Deloukas $(2013)^{4}$ & $\begin{array}{l}\text { Artherosclerotic Disease, Vascular Function \& Genetic } \\
\text { Epidemiology study (ADVANCE), The academic Medical } \\
\text { Center Amsterdam Premature Atherosclerosis Study } \\
\text { (AMC-PAS), Angio-Lueb/KORAF3, Cardiogenics study } \\
\text { (CARDIOGENICS), The Dietary, lifestyle and genetic } \\
\text { determinants of obesity and metabolic syndrome study } \\
\text { (DILGOM), DUKE, Estonian genome center of university } \\
\text { of Tartu (EGCUT GWAS, EGCUT metabochip), The } \\
\text { European prospective investigation into cancer (EPIC), } \\
\text { Functional genomic diagnostic tools for coronary artery } \\
\text { disease (FGENTCARD), Fragmin and Fast } \\
\text { Revascularization (FRISCII-GLACIER), Gene x lifestyle } \\
\text { interactions and compmlex traits involed in elevated disease } \\
\text { risk (GLACIER), The genetics of diabetes audit and } \\
\text { research in Tayside Scotland (GoDARTS), MRC/BHF } \\
\text { heart protection study (HPS), The INTERHEART study } \\
\text { (ITH), London life sciences population study } \\
\text { (LOLIPOP), Ludwigshafen Risk and cardiovascular } \\
\text { health study and echonicoccus Multilocularis and } \\
\text { internal diseases in Leutkirch study (LURIC-EMIL), } \\
\text { METabolic Syndrome In Men (METSIM), Monica, Risk, } \\
\text { Genetics, Archiving and monograph (MORGAM-FIN, } \\
\text { MORGAM-FRA, MORGAM-GER, MORAGM-ITA, } \\
\text { MORGAM-UNK), OHGS, Prospective Investigation of } \\
\text { the Vasculature in Uppsala Seniors (PIVUS), Pfizer- } \\
\text { MGH-Broad (PMD), PopGEN, European collaborative } \\
\text { study of the genetics of precocious coronary artery } \\
\text { disease (PROCARDIS), The Pakistan Risk of Myocardial } \\
\text { Infarction Study (PROMIS GWAS, PROMIS Metabochip), } \\
\text { SCARF-SHEEP, Swedish Twin Registry (STR), The }\end{array}$ & Nelson $(2017)^{1}$ \\
\hline
\end{tabular}


Divers $(2017)^{11}$

Erdmann (2010) ${ }^{13}$ Howson $(2017)^{14}$

Kozian $(2010)^{15}$

Lu $(2014)^{16}$

Lettre $(2011)^{17}$

Nikpay $(2015)^{18}$

O’Donnell (2011) $)^{19}$

Pfister $(2011)^{20}$

Schunkert (2011)
Hellenic study of interactions between Snps and eating

in atherosclerosis susceptibility (THISEAS), Uppsala

longitudinal study of adult men (ULSAM), WTCCC CAD

2, COROGENE, The Finnish cardiovascular study

(FINCAVAS), Genomics Research in Cardiovascular

disease (GenRIC)

African American Diabetes Heart Study (AA-DHS), Raffield (2015) ${ }^{12}$

Jackson Heart Study

German myocardial infarction family study (GerMIFS III) Nelson (2017) ${ }^{1}$

The Copenhagen Ischaemic Heart Disease Study

Nelson $(2017)^{1}$

(CIHDS), The Copenhagen General Population Study

(CGPS), Copenhagen City Heart Study (CCHS), EPIC-

CVD, Bangladesh Risk of Acute Vascular Events

(BRAVE), PROMIS, ARIC, Women's Health Initiative

(WHI), Myocardial infarction genetics consortium

(MIGen), TAlwan metaboCHIp Consortium (TAICHI)

LURIC

Beijing Atherosclerosis Study (BAS), China

Nelson (2017) ${ }^{1}$

Nelson $(2017)^{1}$

atherosclerosis study (CAS), CARDIOGRAM cohorts,

China CHS, China Collaborative Study of Cardiovascular

Epidemiology

ARIC*, The coronary artery risk development in young

Nelson $(2017)^{1}$

adults (CARDIA), Cleveland family study (CFS), Jackson

heart Study (JHS) and Multi-Ethnic Study of Atherosclerosis

(MESA)

ADVANCE, AGES*, ARIC*, BAS (Beijing atherosclerosis Nelson (2017)

study), CARDIOGENICS, CAS, CCGB, COROGENE,

DUKE, EGCUT, Family Heart Study (FamHS)*,

FGENTCARD, FHS, GenRIC, GerMIFS I-IV, GoDARTS,

MRC/BHF Heart Protection Study (HPS), HSDSS, BioMe

Biobank Program, INTERHEART, LIFE-Heart, LOLIPOP,

LURIC, case control study (MAYO-VDB), Medstar

cardiac catheterization study (MedStar), MIGen, OHGS,

Univeristy of Pennsylvania Medical Center cardiac

catherization study (PennCATH), PIVUS, A subset of

FINRISK cohort study (PREDICTCVD), PROCARDIS,

PROMIS, Prospective study of pravastatin in the elderly

at risk (PROSPER)*, Rotterdam Study*, THISEAS,

TWINGENE, ULSAM, Women's genome health study

(WGHS), WTCCC

Cohorts for heart and aging research in genomic

Nelson $(2017)^{1}$

epidemiology (CHARGE), genetic epidemiology network of

arteriopathy study (GENOA)

EPIC Norfolk

Nelson $(2017)^{1}$

ADVANCE, Coronary artery disease and omics

Nelson $(2017)^{1}$

collaborative analysis of diagnostic criteria in Europe

(deCODE CAD), GerMIFS I, GerMIFS II, GerMIFS III,

LURIC/AtheroRemo 1, LURIC/AtheroRemo 2, MedStar,

MIGen, OHGS1, PennCATH, WTCCC, Acute Myocardial

Infarction Gene Study / Dortmund Health Study (AMI/DHS),

AMC-PAS, Angio-Lueb/Gokard, CHAOS, Cleveland Clinic

GeneBank/OHGS2, EPIC-CAD, GENDER, GraceGenetics, 
Stark (2009) ${ }^{21}$

The IBC 50K CAD

Consortium $(2011)^{22}$

Trégouët (2009) ${ }^{23}$

Varbo $(2011)^{5}$

Wang $(2011)^{24}$

Webb $(2017)^{26}$

Wild $(2012)^{27}$

Willer (2008) $)^{28}$

Yaghootkar $(2014)^{29}$

Zhao $(2017)^{2}$
INTERHEART, Intermountain Heart Collaborative Study (IHCS), Irish Family Study (IFS), Italian atherosclerosis, thrombosis and vascular biology study (IATVB),

LEEDS, Malmo Diet and cancer study-cardiovascular cohort (MDCS), Mid-America Mid-America Heart Insitute (MAHI), PopGen, SAS, Study of Myoarcial Infarction in Leiden (SMILE), SHEEP, The Emory Genebank Study, The Johns Hopkins GeneSTAR Research Program, The New Zealand CAD Study, THISEAS, UKMI, Verona Heart Study (VHS)

German MI Family Study

Nelson $(2017)^{1}$

ARIC ${ }^{*}$, BLOODOMICS-Dutch (AMC-PAS + AGNES), $\quad$ Nelson (2017) ${ }^{1}$

BLOODOMICS-German LURIC + Mannheim), British Heart

Foundation Family Heart Study (BHF-FHS), The

cardiovascular Health Study (CHS), CARDIA, FHS,

LOLIPOP, MONICA-KORA, PennCATH, PROCARDIS, PROMIS

WTCCC

CCHS, CGPS, CIHDS

GenelD population China

Nelson (2017)

Nelson $(2017)^{1}$

Lian $(2013)^{25}$

ATVB, BHF-FHS, Vanderbilt University Medical Center Nelson (2017) Biorepository (BioVU), DUKE, EPIC, First-time incidence of myocardial infarction in the AC county 3 (FIA3), GoDARTS, EGCUT, German CAD North, German CAD South, Nord-Trondelag health study (HUNT), BioMe Biobank, MDC, Montreal heart institute study (MHI), OHS, PAS-AMC, PennCath, PROCARDIS, VHS, WHI

The Gutenberg Heart Study (GHS), Atherogene Registry, Gutenberg Heart Express Study (GHSExpress), CHARGE, GerMIFSI, GerMIFSII, MedStar, PennCATH, The MIGen consortium, WTCCC-CAD, AngioLueb, Etude Cas-Témoin sur l'Infarctus du Myocarde (ECTIM), LURIC, MORGAM,

Popgen

WTCCC

ADVANCE, CADomics, CHARGE, DeCode, GERMifs, Nelson (2017) ${ }^{1}$ LURIC, MedStar, MIGen, OHGS, PennCATH, WTCCC

PROMIS, Risk Assessment of cerebrovascular events study Nelson (2017) (RACE), BRAVE, A prospective cohort to determine evnrionment and genetic determinants of metabolic syndrome related factors (EPIDREAM), FINRISK, MedStar, MDC, PennCATH, LOLIPOP, The Singapore Indian Eye Study (SINDI), The Khatri Sikh Diabetes Study (SDS), TAICHI, BioBank Japan (BBJ)

*Cohorts AGES, ARIC, FamHS, FHS, PROSPER and Rotterdam Study are part of the CHARGE consortium. 
Table S3.4 Quality assessment of the CAD studies based on the Newcastle-Ottawa Scale (NOS).

\begin{tabular}{|c|c|c|c|c|c|c|c|c|c|}
\hline References & & cti & & & Comparability & & $\begin{array}{l}\text { surel } \\
\text { ome }\end{array}$ & & $\begin{array}{l}\text { Quality } \\
\text { judgment }\end{array}$ \\
\hline & 1 & 2 & 3 & 4 & 1 & 1 & 2 & 3 & \\
\hline Lian $(2013)^{25}$ & $\bar{x}$ & $\bar{t}$ & & 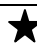 & $\star \star \star x$ & $\star$ & 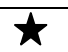 & 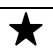 & \\
\hline Nelson $(2017)^{1}$ & $\hbar$ & & $\star$ & & & $\star$ & $\star$ & 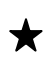 & \\
\hline Raffield $(2015)^{12}$ & & & $\star$ & & $\star$ & & $\star$ & & t \\
\hline Takeuchi $(2012)^{30}$ & 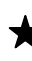 & t & $\star$ & $\star$ & & $\star$ & $\star$ & $\star$ & \\
\hline Zhou $(2015)^{31}$ & 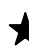 & $\lambda$ & $\star$ & $\star \lambda$ & & $\star$ & $\star \star$ & $\star$ & \\
\hline
\end{tabular}

Notes case-control studies (i.e., Lian (2013), Takeuchi (2012), Zhou (2015)): categories of the quality assessment are displayed in bold, with interpretation of each item within the categories for this specific metaanalysis placed between brackets.

Selection: 1. Is the case definition adequate? (if yes, with independent validation (e.g., hospital records), one star; if yes, with record linkage (e.g., ICD-10 code or self-report) or no description, no star); 2. Representativeness of the cases (if consecutive or obviously representative series of cases, one star; if not consecutive or not (clearly) stated, no star); 3. Selection of controls (if community controls, one star; if hospital controls or no description, no star); 4. Definition of controls (if yes, with 'no history of CAD' explicitly stated, one star; if 'no history of CAD' not explicitly stated or no description, no star). Comparability: 1. Comparability of cases and controls on the basis of the design or analysis (if study adjusts for no covariates, two stars, if study adjusts for age and/or gender only, one star, if study adjusts for more covariates than age and/or gender, no star). Exposure: 1. Ascertainment of exposure (if secure record (e.g., genotyping), one star; if no description, no star); 2. Same method of ascertainment for cases and controls (if yes, one star; if no or no description, no star); 3. Non-response rate (if same rate for both groups, one star; if rate differs for both groups or no designation, no star).

Notes cohort studies (i.e., Nelson (2017), Raffield (2015)): categories of the quality assessment are displayed in bold, with interpretation of each item within the categories for this specific meta-analysis placed between brackets.

Selection: 1. Representativeness of the exposed cohort (if truly or somewhat representative of the average population, one star; if selected group (e.g., patients with type 2 diabetes) or no description, no star). 2. Selection of the non-exposed cohort (if drawn from the same community as the exposed cohort, one star; if drawn from a different source or no description, no star). 3. Ascertainment of exposure (if secure record (e.g., genotyping), one star; if no description, no star). 4. Demonstration that outcome of interest was not present at the start of the study (if explicitly stated, one star; if not explicitly stated, no star). Comparability: 1. Comparability of cases and controls on the basis of the design or analysis (if study adjusts for no covariates, two stars, if study adjusts for age and/or gender only, one star, if study adjusts for more covariates than age and/or gender, no star). Outcome: 1. Assessment of outcome (if independent blind assessment or record linkage (e.g., hospital records), one star; if with record linkage (e.g., ICD-10 code or self-report) or no description, no star). 2. Was follow-up long enough for outcome to occur (if average age of the sample population minus two standard deviations was equal to or more than 40 years, one star; if average age of the sample population minus two standard deviations was less than 40 years, no star). 3. Adequacy of follow up of cohorts (if explicitly stated why subjects from original cohort were excluded, one star; if not explicitly stated why subjects from original cohort were excluded, no star). 
Table S3.5 Overview of the excluded eGFR and CKD studies with duplicate cohorts.

\begin{tabular}{|c|c|c|}
\hline Excluded study & Study cohorts (with duplicate cohort in bold) & $\begin{array}{l}\text { Study in which } \\
\text { duplicate cohort is } \\
\text { included }\end{array}$ \\
\hline Gorski $(2015)^{32}$ & $\begin{array}{l}\text { Age, Gene/Environment susceptibility-Reykjavik Study } \\
\text { (AGES) }^{*} \text {, Amish Studies, The atherosclerosis risk in } \\
\text { communities study (ARIC)*, Austrian stroke } \\
\text { prevention study (ASPS), Cardiovascular health study } \\
\text { (CHS), The Cohort Lausannoise study (CoLaus), } \\
\text { Framingham Heart Study (FHS), Genetic epidemiology } \\
\text { network of arteriopathy (GENOA), Health aging and } \\
\text { body composition study (HABC), JUPITER, } \\
\text { Cooperative research in the region of Augsburg } \\
\text { (KORA), multi-ethnic study of atherosclerosis (MESA), } \\
\text { Rotterdam Study, Study of health in Pomerania (SHIP), } \\
\text { Three Cities (3C) }\end{array}$ & Pattaro $(2016)^{33}$ \\
\hline Köttgen (2009) & $\begin{array}{l}\text { Cohorts for heart and aging research in genomic } \\
\text { epidemiology (CHARGE) which includes ARIC*, CHS, } \\
\text { FHS, Rotterdam Study }\end{array}$ & Pattaro $(2016)^{33}$ \\
\hline Köttgen $(2010)^{35 * *}$ & $\begin{array}{l}\text { AGES*, Amish studies, ARIC*, ASPS, Baltimore } \\
\text { longitudinal study of aging (BLSA), CHS, Erasmus } \\
\text { rucphen family (ERF), FamHS*, FHS, GENOA, } \\
\text { Gutenburg Heart Study (GHS), KORA F3 and F4, } \\
\text { Korcula Croatia, Microisolates in South tyrol study } \\
\text { (MICROS), The northern Swedish population health } \\
\text { study (NSPHS), Orkney complex disease study } \\
\text { (ORCADES), Rotterdam Study, SHIP, VIS CROATIA, } \\
\text { Women's genome health study (WGHS), HABC, Health } \\
\text { professionals follow-up study (HPFS), Nurses health } \\
\text { study (NHS), POPGen, The Sorbs study (Sorbs), } \\
\text { SPLIT, Swiss study on air polluation and lung } \\
\text { diseases in adults (SAPALDIA), Salzburg } \\
\text { Atherosclerosis prevention program in subjects at } \\
\text { high individual risk (SAPHIR) }\end{array}$ & Pattaro $(2016)^{33}$ \\
\hline Lanktree $(2018)^{36}$ & $\begin{array}{l}\text { Atherosclerotic Disease, Vascular function, \& Genetic } \\
\text { epidemiology study (ADVANCE), The academic medical } \\
\text { center of Amsterdam Premature Atherosclerosis Cohort } \\
\text { (AMC-PAS), AMISH, } 1958 \text { British Birth Cohort (BC58), } \\
\text { D2D } 2007 \text { (D2D), deCODE, The diabetes Genetic Study } \\
\text { (DIAGEN), The dietary, lifestyle and genetic determinants } \\
\text { of obesity and metabolic syndrome study (DILGOM), The } \\
\text { finnish diabetes prevention study (DPS), The dose } \\
\text { responses to exercise training study (DR'S EXTRA), } \\
\text { Edinburgh Artery Study (EAS), Estonian genome center } \\
\text { of university of Tartu (EGCUT), Ely, The european } \\
\text { prospective investigation into cancer and nutrition (EPIC- } \\
\text { CAD), Fenland, The Finnish cardiovascular study } \\
\text { (FINCAVAS), Fragmin and fast revascularization during } \\
\text { instability in coronary artery disease (FRISCII), FUSION2, } \\
\text { Gene x lifestyle interactions and complex traits involved in } \\
\text { elevated disease risk (GLACIER), Genetics of diabetes } \\
\text { and audit research Tayside (Go-DARTs), Nord- } \\
\text { Trondelag health study } 2 \text { (HUNT), IMPROVE, KORA F3 }\end{array}$ & $\begin{array}{l}\text { Pattaro }(2016)^{33} \\
\text { Deshmukh }(2013)^{37}\end{array}$ \\
\hline
\end{tabular}




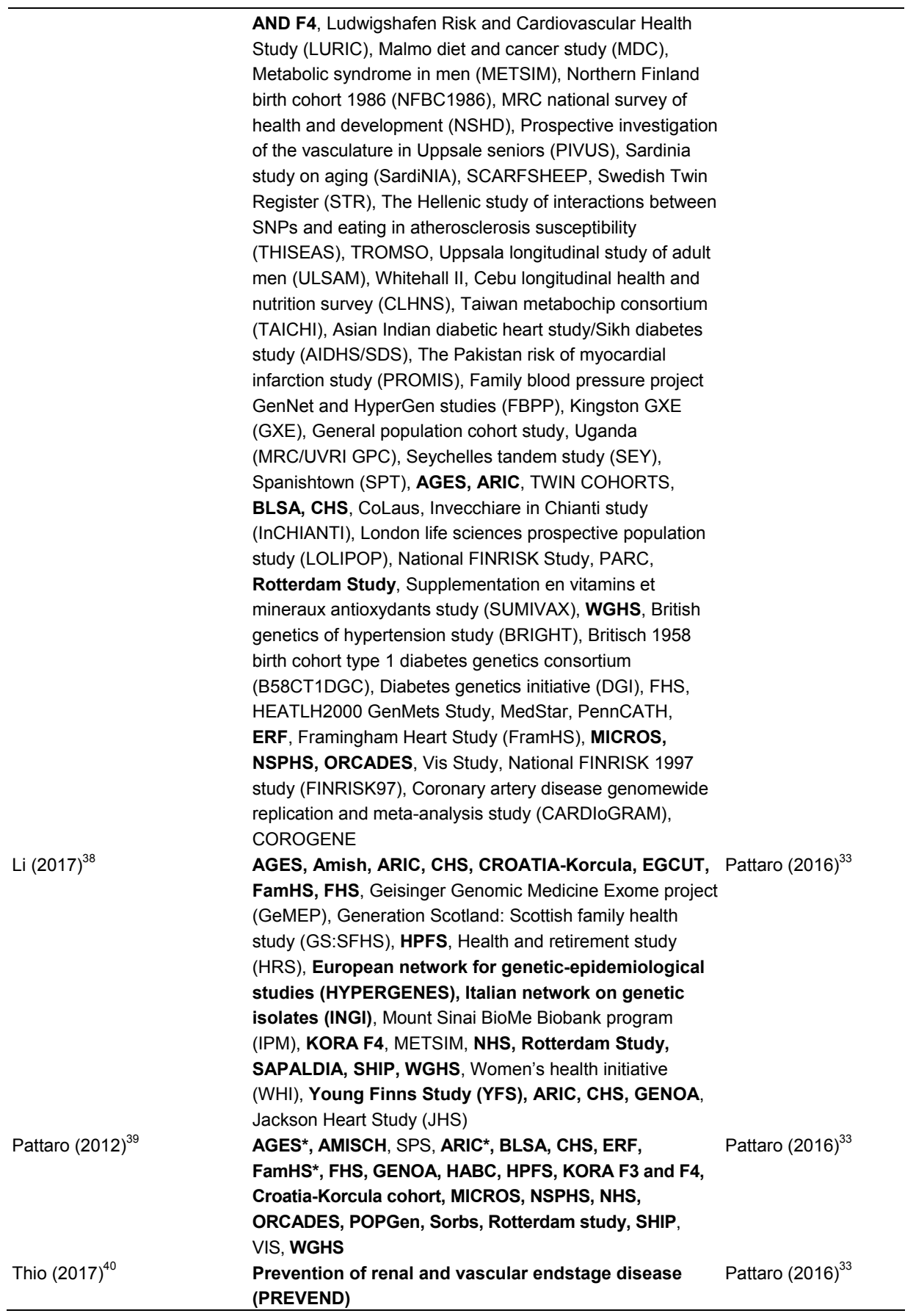

*Cohorts AGES, ARIC, FamHS, FHS, PROSPER and Rotterdam Study are part of the CHARGE consortium. **This study was included for the data-synthesis on cystatin C-based eGFR, since this was not reported by Pattaro (2016). ${ }^{33}$ 
Table S3.6 Quality assessment of the eGFR and CKD studies based on the NOS.

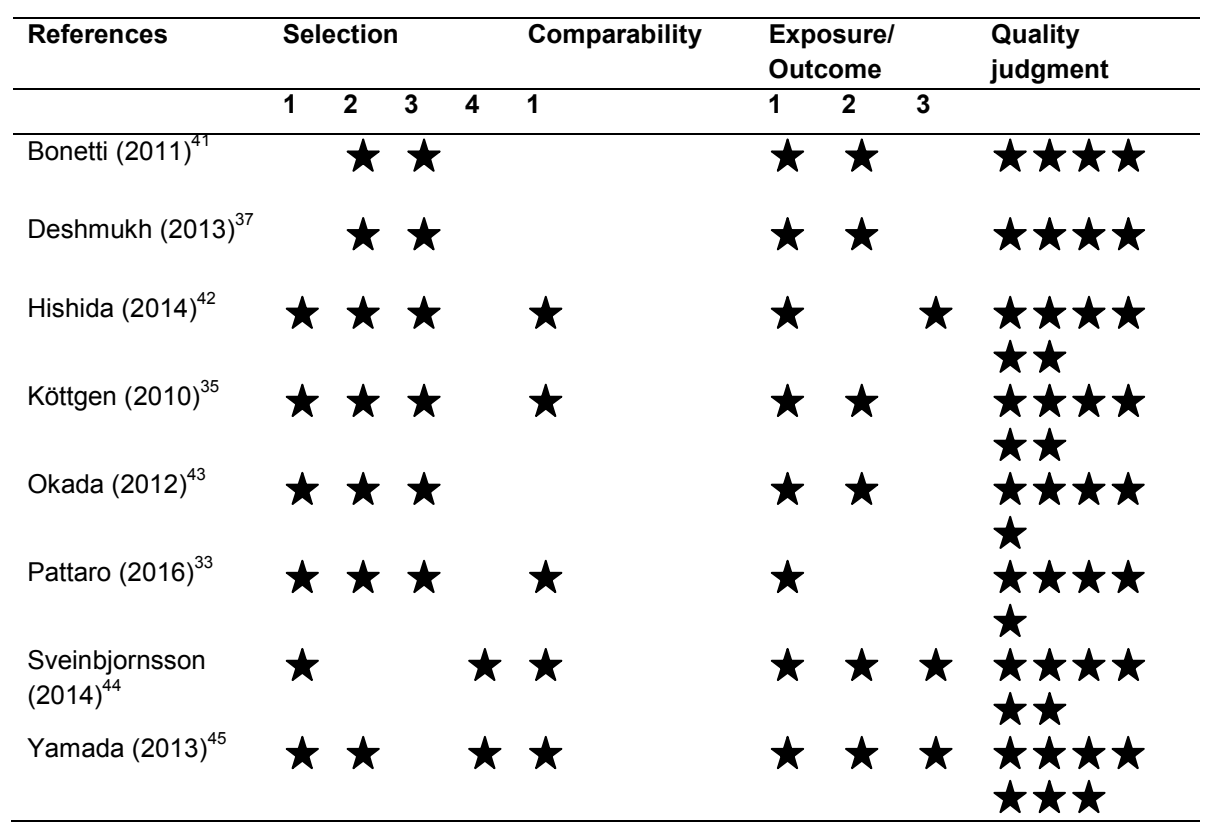

Notes case-control studies (i.e., Sveinbjornsson (2014), Yamada (2013)): categories of the quality assessment are displayed in bold, with interpretation of each item within the categories for this specific meta-analysis placed between brackets.

Selection: 1. Is the case definition adequate? (if yes, with independent validation (e.g., hospital records), one star; if yes, with record linkage (e.g., ICD-10 code or self-report) or no description, no star); 2. Representativeness of the cases (if consecutive or obviously representative series of cases, one star; if not consecutive or not (clearly) stated, no star); 3. Selection of controls (if community controls, one star; if hospital controls or no description, no star); 4. Definition of controls (if yes, with 'no history of CKD' explicitly stated, one star; if 'no history of CKD' not explicitly stated or no description, no star). Comparability: 1. Comparability of cases and controls on the basis of the design or analysis (if study adjusts for no covariates, two stars, if study adjusts for age and/or gender only, one star, if study adjusts for more covariates than age and/or gender, no star). Exposure: 1. Ascertainment of exposure (if secure record (e.g., genotyping), one star; if no description, no star); 2. Same method of ascertainment for cases and controls (if yes, one star; if no or no description, no star); 3. Non-response rate (if same rate for both groups, one star; if rate differs for both groups or no designation, no star).

Notes cohort studies (i.e., Bonetti (2011), Deshmukh (2013), Hishida (2014), Köttgen (2010), Okado (2012), Pattaro (2016)): categories of the quality assessment are displayed in bold, with interpretation of each item within the categories for this specific meta-analysis placed between brackets.

Selection: 1. Representativeness of the exposed cohort (if truly or somewhat representative of the average population, one star; if selected group (e.g., patients with type 2 diabetes) or no description, no star). 2. Selection of the non-exposed cohort (if drawn from the same community as the exposed cohort, one star; if drawn from a different source or no description, no star). 3. Ascertainment of exposure (if secure record (e.g., genotyping), one star; if no description, no star). 4. Demonstration that outcome of interest was not present at the start of the study (if dichotomous variable (e.g., CKD yes/no) and 'no history of CKD' explicitly stated, one star; if dichotomous variable (e.g., CKD yes/no) and 'no history of CKD' not explicitly stated, or continuous variable (e.g., eGFR), no star). Comparability: 1. Comparability of cases and controls on the basis of the design or analysis (if study 
adjusts for no covariates, two stars, if study adjusts for age and/or gender only, one star, if study adjusts for more covariates than age and/or gender, no star). Outcome: 1. Assessment of outcome (if independent blind assessment or record linkage (e.g., hospital records), one star; if with record linkage (e.g., ICD-10 code or selfreport) or no description, no star). 2. Was follow-up long enough for outcome to occur (if average age of the sample population minus two standard deviations was equal to or more than 40 years or variable was continuous (e.g., eGFR), one star; if average age of the sample population minus two standard deviations was less than 40 years, no star). 3. Adequacy of follow up of cohorts (if explicitly stated why subjects from original cohort were excluded, one star; if not explicitly stated why subjects from original cohort were excluded, no star). 


\section{Literature}

1. Nelson CP, Goel A, Butterworth AS, et al. Association analyses based on false discovery rate implicate new loci for coronary artery disease. Nat Genet 2017;49:1385-91.

2. Zhao $B$, Lu $Q$, Cheng $Y$, et al. A Genome-wide association study to identify single-nucleotide polymorphisms for acute kidney injury. Am J Respir Crit Care Med 2017;195:482-90.

3. Schunkert H, Konig IR, Kathiresan S, et al. Large-scale association analysis identifies 13 new susceptibility loci for coronary artery disease. Nat Genet 2011;43:333-8.

4. Deloukas $\mathrm{P}$, Kanoni $\mathrm{S}$, Willenborg $\mathrm{C}$, et al. Large-scale association analysis identifies new risk loci for coronary artery disease. Nat Genet 2013;45:25-33.

5. Varbo A, Benn M, Tybjaerg-Hansen A, Grande P, Nordestgaard BG. TRIB1 and GCKR polymorphisms, lipid levels, and risk of ischemic heart disease in the general population. Arterioscler Thromb Vasc Biol 2011;31:451-7.

6. Angelakopoulou A, Shah T, Sofat R, et al. Comparative analysis of genome-wide association studies signals for lipids, diabetes, and coronary heart disease: Cardiovascular Biomarker Genetics Collaboration. Eur Heart J 2012;33:393-407.

7. Bi M, Kao WH, Boerwinkle E, et al. Association of rs780094 in GCKR with metabolic traits and incident diabetes and cardiovascular disease: the ARIC Study. PLoS One 2010;5:e11690.

8. The Wellcome Trust Case Control C. Genome-wide association study of 14,000 cases of seven common diseases and 3,000 shared controls. Nature 2007;447:661.

9. Davies RW, Wells GA, Stewart AF, et al. A genome-wide association study for coronary artery disease identifies a novel susceptibility locus in the major histocompatibility complex. Circ Cardiovasc Genet 2012;5:217-25.

10. Dehghan A, Bis JC, White CC, et al. Genome-Wide Association Study for Incident Myocardial Infarction and Coronary Heart Disease in Prospective Cohort Studies: The CHARGE Consortium. PLoS One 2016;11:e0144997.

11. Divers J, Palmer ND, Langefeld CD, et al. Genome-wide association study of coronary artery calcified atherosclerotic plaque in African Americans with type 2 diabetes. BMC Genet 2017;18 (1) (no pagination).

12. Raffield LM, Cox AJ, Carr JJ, et al. Analysis of a cardiovascular disease genetic risk score in the Diabetes Heart Study. Acta Diabetol 2015;52:743-51.

13. Erdmann J, Willenborg C, Nahrstaedt J, et al. Genome-wide association study identifies a new locus for coronary artery disease on chromosome 10p11.23. Eur Heart J 2011;32: $158-68$.

14. Howson JMM, Zhao W, Barnes DR, et al. Fifteen new risk loci for coronary artery disease highlight arterial-wall-specific mechanisms. Nat Genet 2017;49:1113-9.

15. Kozian DH, Barthel A, Cousin E, et al. Glucokinase-activating GCKR polymorphisms increase plasma levels of triglycerides and free fatty acids, but do not elevate cardiovascular risk in the Ludwigshafen Risk and Cardiovascular Health Study. Horm Metab Res 2010;42:502-6.

16. Sevastianova K, Santos A, Kotronen A, et al. Effect of short-term carbohydrate overfeeding and long-term weight loss on liver fat in overweight humans. Am J Clin Nutr 2012;96:727-34.

17. Lettre G, Palmer CD, Young T, et al. Genome-Wide Association Study of Coronary Heart Disease and Its Risk Factors in 8,090 African Americans: The NHLBI CARe Project. PLoS Genet 2011;7:e1001300.

18. Nikpay M, Goel A, Won HH, et al. A comprehensive 1,000 Genomes-based genome-wide association meta-analysis of coronary artery disease. Nat Genet 2015;47:1121-30.

19. Speliotes EK, Yerges-Armstrong LM, Wu J, et al. Genome-wide association analysis identifies variants associated with nonalcoholic fatty liver disease that have distinct effects on metabolic traits. PLoS Genet 2011;7:e1001324. 
20. Pfister R, Barnes D, Luben RN, Khaw KT, Wareham NJ, Langenberg C. Individual and cumulative effect of type 2 diabetes genetic susceptibility variants on risk of coronary heart disease. Diabetologia 2011;54:2283-7.

21. Stark K, Reinhard W, GrassI M, et al. Common Polymorphisms Influencing Serum Uric Acid Levels Contribute to Susceptibility to Gout, but Not to Coronary Artery Disease. PLoS One 2009;4:e7729.

22. The IBCKCADC. Large-Scale Gene-Centric Analysis Identifies Novel Variants for Coronary Artery Disease. PLoS Genet 2011;7:e1002260.

23. Tregouet DA, Konig IR, Erdmann J, et al. Genome-wide haplotype association study identifies the SLC22A3-LPAL2-LPA gene cluster as a risk locus for coronary artery disease. Nat Genet 2009;41:283-5.

24. Wang $F, X u C Q, H e Q$, et al. Genome-wide association identifies a susceptibility locus for coronary artery disease in the Chinese Han population. Nat Genet 2011;43:345-9.

25. Lian J, Guo J, Chen Z, et al. Positive association between GCKR rs780093 polymorphism and coronary heart disease in the aged Han Chinese. Dis Markers 2013;35:863-8.

26. Webb TR, Erdmann J, Stirrups KE, et al. Systematic Evaluation of Pleiotropy Identifies 6 Further Loci Associated With Coronary Artery Disease. J Am Coll Cardiol 2017;69:823-36.

27. Wild PS, Zeller T, Schillert A, et al. A genome-wide association study identifies LIPA as a susceptibility gene for coronary artery disease. Circ Cardiovasc Genet 2011;4:403-12.

28. Willer CJ, Sanna S, Jackson AU, et al. Newly identified loci that influence lipid concentrations and risk of coronary artery disease. Nat Genet 2008;40:161-9.

29. Yaghootkar H, Scott RA, White CC, et al. Genetic evidence for a normal-weight "metabolically obese" phenotype linking insulin resistance, hypertension, coronary artery disease, and type 2 diabetes. Diabetes 2014;63:4369-77.

30. Takeuchi F, Isono M, Katsuya $T$, et al. Association of genetic variants influencing lipid levels with coronary artery disease in Japanese individuals. PLoS One 2012;7:e46385.

31. Zhou YJ, Hong SC, Yin RX, Yang Q, Cao XL, Chen WX. Polymorphisms in the GCKR are associated with serum lipid traits, the risk of coronary artery disease and ischemic stroke. Int J Clin Exp Med 2015;8:10678-86.

32. Gorski M, Tin A, Garnaas M, et al. Genome-wide association study of kidney function decline in individuals of European descent. Kidney Int 2015;87:1017-29.

33. Pattaro C, Teumer A, Gorski M, et al. Genetic associations at 53 loci highlight cell types and biological pathways relevant for kidney function. Nat Commun 2016;7:10023.

34. Köttgen A, Glazer NL, Dehghan A, et al. Multiple Novel Loci are Associated with Indices of Renal Function and Chronic Kidney Disease. Nat Genet 2009;41:712-7.

35. Kottgen A, Pattaro C, Boger CA, et al. New loci associated with kidney function and chronic kidney disease. Nat Genet 2010;42:376-84.

36. Lanktree MB, Theriault S, Walsh M, Pare G. HDL Cholesterol, LDL Cholesterol, and Triglycerides as Risk Factors for CKD: A Mendelian Randomization Study. Am J Kidney Dis 2018;71:166-72.

37. Deshmukh HA, Palmer CN, Morris AD, Colhoun HM. Investigation of known estimated glomerular filtration rate loci in patients with type 2 diabetes. Diabet Med 2013;30:1230-5.

38. Li M, Li Y, Weeks O, et al. SOS2 and ACP1 Loci Identified through Large-Scale Exome Chip Analysis Regulate Kidney Development and Function. Journal of the American Society of Nephrology : JASN 2017;28:981-94.

39. Pattaro C, Köttgen A, Teumer A, et al. Genome-Wide Association and Functional Follow-Up Reveals New Loci for Kidney Function. PLoS Genet 2012;8:e1002584.

40. Thio CHL, van der Most PJ, Nolte IM, et al. Evaluation of a genetic risk score based on creatinine-estimated glomerular filtration rate and its association with kidney outcomes. Nephrol Dial Transplant 2018;33(10):1757-64.

41. Bonetti S, Trombetta M, Boselli ML, et al. Variants of GCKR affect both beta-cell and kidney function in patients with newly diagnosed type 2 diabetes: the Verona newly diagnosed type 2 diabetes study 2. Diabetes Care 2011;34:1205-10. 
42. Hishida A, Takashima N, Turin TC, et al. GCK, GCKR polymorphisms and risk of chronic kidney disease in Japanese individuals: data from the J-MICC Study. J Nephrol 2014;27: 143-9.

43. Okada $Y, \operatorname{Sim} X$, Go MJ, et al. Meta-analysis identifies multiple loci associated with kidney function-related traits in east Asian populations. Nat Genet 2012;44:904-9.

44. Sveinbjornsson G, Mikaelsdottir E, Palsson R, et al. Rare mutations associating with serum creatinine and chronic kidney disease. Hum Mol Genet 2014;23:6935-43.

45. Yamada $Y$, Nishida T, Ichihara S, et al. Identification of chromosome 3q28 and ALPK1 as susceptibility loci for chronic kidney disease in Japanese individuals by a genome-wide association study. J Med Genet 2013;50:410-8. 


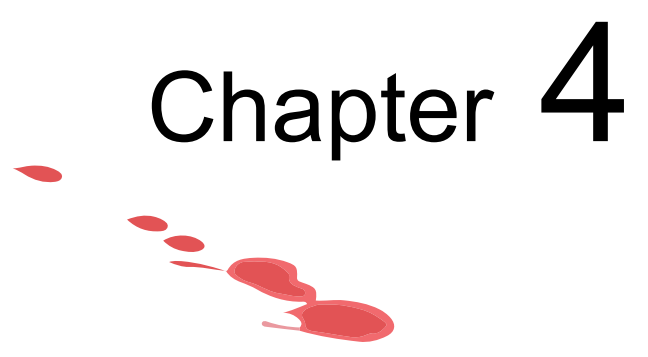

Effects of genetic variants in TM6SF2, PNPLA3 and MBOAT7 on coronary artery disease

PNPLA3, TM6SF2 and MBOAT7 genotypes and coronary artery disease Nynke Simons, Aaron Isaaes, Martijn C.G.J. Brouwers Gastroenterology. 2017;152(4):912-913 
Chapter 4 


\section{Letter to the editor}

Nonalcoholic fatty liver disease (NAFLD) is projected to be the most common cause of end-stage liver disease requiring transplantation by $2025 .{ }^{1}$ The principal cause of death, however, is cardiovascular disease. In a recent metaanalysis, NAFLD - as diagnosed by either imaging or histology - was associated with an increased risk to develop cardiovascular disease, independent of all classical cardiovascular risk factors (OR: $1.69,95 \% \mathrm{Cl}$ : $1.11 ; 2.58)^{2}$

Genetic epidemiology may be helpful to unveil the biological pathways that relate NAFLD to cardiovascular disease. We and others have previously shown that up to $35 \%$ of the variability in NAFLD can be explained by genetic factors. ${ }^{3}$ To date, two common genetic variants have consistently been associated with all histological stages of NAFLD (ranging from simple steatosis to NASH and fibrosis), i.e., patatin-like phospholipase domain-containing protein 3 (PNPLA3) and transmembrane 6 superfamily 2 (TM6SF2). ${ }^{4}$ More recently, the membranebound O-acyltransferase domain-containing protein 7 (MBOAT7) gene was identified as a new NAFLD susceptibility gene. ${ }^{5}$

Previous studies have already demonstrated that NAFLD risk and cardiovascular risk do not coincide in carriers of the TM6SF2 minor allele. ${ }^{4}$ In the present study, we sought to assess the risk of coronary artery disease (CAD) conferred by PNPLA3 and MBOAT7.

Data on coronary artery disease were contributed by CARDIoGRAMplusC4D investigators and downloaded from www.CARDIOGRAMPLUSC4D.org (Coronary Artery Disease Genome wide Replication and Meta-analysis [CARDIoGRAM] plus the Coronary Artery Disease [C4D]). This cohort contains data from 48 genome wide association studies including 60,801 cases with CAD and 123,504 controls. ${ }^{6}$

Statistical analyses showed that the rs738409 G-allele (PNPLA3) was associated with a modest protection from CAD in an additive genetic model, albeit not statistically significant (OR: $0.98 ; 95 \% \mathrm{Cl}$ : $0.96 ; 1.00)$. The recessive model, on the other hand, did reach significance (OR: $0.92 ; 95 \% \mathrm{Cl}: 0.87 ; 0.97$, Table 4.1). The rs5854292 T-allele (TM6SF2) also protected from CAD in the CARDIoGRAMplusC4D consortium, which is in corroboration with previous studies. ${ }^{4}$ In contrast, the rs641738 T-allele (MBOAT7) exerted a neutral effect on CAD risk in either genetic model (Table 4.1). 
Chapter 4

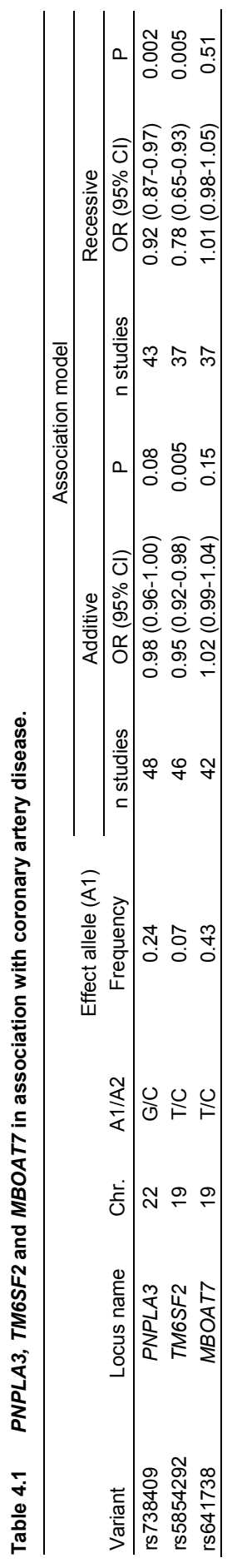


At first sight, these observations appear to be in contradiction with a recent meta-analysis that reported a positive relationship between NAFLD and cardiovascular disease, even after adjustment for established cardiovascular risk factors. ${ }^{2}$ This discrepancy may be explained by differences in the metabolic pathways that all result in NAFLD, but have a distinctive impact on cardiovascular risk.

Stable isotope studies have shown that an increased flux of free fatty acids towards the liver and de novo lipogenesis are the two principal pathways that cause NAFLD. ${ }^{7}$ The genes under investigation in the current study - which account for only a small proportion in the total variability of NAFLD - are likely to affect completely different pathways. Carriers of the TM6SF2 minor allele are for instance characterized by impaired VLDL secretion and consequently NAFLD and hypolipidemia. ${ }^{4}$ Given the pivotal role that plasma lipids play in the pathogenesis of atherosclerosis it is self-evident that NAFLD risk and CAD risk do not coincide in case of TM6SF2. The same explanation may hold true for PNPLA3. The current concept is that the rs738409 G-allele encodes a PNPLA3 protein (I148M) with decreased hydrolyse activity. As a consequence, intrahepatic triglyceride breakdown is hampered, which subsequently may affect VLDL particle production and secretion. ${ }^{4}$ Indeed, the PNPLA3 minor allele has been associated with decreased plasma lipids in some but not all studies. $^{8}$

This would explain the modest, negative association between PNPLA3 and CAD under the assumption of a recessive model in the current study. Finally, the exact role of the recently discovered MBOAT7 in the pathogenesis and progression of NAFLD remains to be elucidated. MBOAT7 is an acyltransferase that facilitates acyl-chain remodelling of phosphatidylinositols. The neutral effects of MBOAT7 on CAD in the present study may relate to the also neutral effects on plasma lipids that were observed in recent studies. ${ }^{5}$

The CARDIoGRAMplusC4D cohort is unique with regard to its large number of individuals with and without CAD in whom common and low frequency variants have been genotyped and imputed at the genome-wide level. ${ }^{6}$ Unfortunately, data on NAFLD or plasma lipids are not available for this cohort. Inferences on biological factors that may mediate the relationship between the hitherto presented genes and CAD can therefore not be made.

In conclusion, although PNPLA3, TM6SF2 and MBOAT7 all predispose to all histological stages of NAFLD, they tend to have different effects on cardiovascular 
risk. These findings suggest that the relationship between NAFLD and cardiovascular disease depend on the biological mechanisms that underlie NAFLD. 


\section{Literature}

1. Charlton MR, Burns JM, Pedersen RA, Watt KD, Heimbach JK, Dierkhising RA. Frequency and outcomes of liver transplantation for nonalcoholic steatohepatitis in the United States. Gastroenterology 2011;141:1249-53.

2. Targher G, Byrne CD, Lonardo A, Zoppini G, Barbui C. Non-alcoholic fatty liver disease and risk of incident cardiovascular disease: A meta-analysis. J Hepatol 2016;65: 589-600.

3. Brouwers MC, van Greevenbroek MM, Cantor RM. Heritability of nonalcoholic fatty liver disease. Gastroenterology 2009;137:1536.

4. Anstee QM, Day CP. The Genetics of Nonalcoholic Fatty Liver Disease: Spotlight on PNPLA3 and TM6SF2. Semin Liver Dis 2015;35:270-90.

5. Mancina RM, Dongiovanni P, Petta S, et al. The MBOAT7-TMC4 Variant rs641738 Increases Risk of Nonalcoholic Fatty Liver Disease in Individuals of European Descent. Gastroenterology 2016;150:1219-30.e6.

6. Nikpay M, Goel A, Won HH, et al. A comprehensive 1,000 Genomes-based genome-wide association meta-analysis of coronary artery disease. Nat Genet 2015;47:1121-30.

7. Donnelly KL, Smith Cl, Schwarzenberg SJ, Jessurun J, Boldt MD, Parks EJ. Sources of fatty acids stored in liver and secreted via lipoproteins in patients with nonalcoholic fatty liver disease. J Clin Invest 2005;115:1343-51.

8. Tang CS, Zhang H, Cheung $\mathrm{CY}$, et al. Exome-wide association analysis reveals novel coding sequence variants associated with lipid traits in Chinese. Nat Commun 2015;6:10206. 



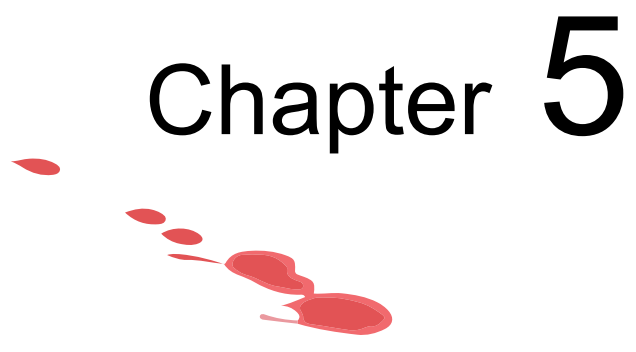

Effects of NAFLD susceptibility genes on coronary artery disease in relation to

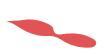

plasma lipids

Relationship between nonalcoholic fatty liver disease susceptibility genes and coronary artery disease

Martijn C.G.J. Brouwers, Nynke Simons, Coen D.A. Stehouwer, Ger-H.Kóek, Nicolaas C. Schaper, Aaron Isaacs Hepatol Commun. 2019;3(4):587-596 


\section{Abstract}

\section{Background}

Coronary artery disease (CAD) is the principal cause of death in patients with nonalcoholic fatty liver disease (NAFLD). The aim of the present study was to investigate whether NAFLD is causally involved in the pathogenesis of CAD.

\section{Methods}

For this, previously reported NAFLD susceptibility genes were clustered and tested for an association with CAD in the Coronary Artery Disease Genomewide Replication and Meta-analysis plus the Coronary Artery Disease (CARDIoGRAMplusC4D) Consortium dataset. The role of plasma lipids as a potential mediator was explored by using data from the Global Lipids Genetics Consortium.

\section{Results}

Statistical analyses revealed that the combination of 12 NAFLD genes was not associated with CAD in 60,801 CAD cases and 123,504 controls (odds ratio [OR] per NAFLD risk allele:1.0; 95\% confidence interval [Cl]: 0.99;1.00). In a subsequent sensitivity analysis, a positive relationship was observed after exclusion of genetic variants that are implicated in NAFLD through impaired very-low-density lipoprotein secretion (i.e., MTTP, PNPLA3, PEMT and TM6SF2) (OR: 1.01; 95\% Cl: 1.00;1.02). Clustering of the excluded genes showed a significant, negative relationship with CAD (OR: $0.97 ; 95 \%$ $\mathrm{Cl}: 0.96 ; 0.99)$. A substantial proportion of the observed heterogeneity between the individual NAFLD genes in relation to CAD could be explained by plasma lipids, as reflected by a strong relationship between plasma lipids and CAD risk conferred by the NAFLD susceptibility genes $(r=0.76 ; p=0.004$ for low-density lipoprotein cholesterol).

\section{Conclusions}

NAFLD susceptibility genes do not cause CAD per se. The relationship between these genes and CAD appears to depend to a large extent on plasma lipids. These observations strongly suggest taking plasma lipids into account when designing a new drug to target NAFLD. 


\section{Background}

Nonalcoholic fatty liver disease (NAFLD) is an emerging cause of progressive liver inflammation, fibrosis, cirrhosis, end-stage liver failure and hepatocellular carcinoma in Western society. It has been estimated that NAFLD will be the principal cause of end-stage liver disease requiring liver transplantation by 2025.

However, despite these alarming data concerning liver-related outcomes, most patients with NAFLD eventually die of cardiovascular complications. ${ }^{2}$ In their meta-analysis, Targher and colleagues recently showed that NAFLD is indeed associated with cardiovascular disease (CVD). ${ }^{3}$ These studies were, by design, not capable of designating NAFLD as either a causal factor or an innocent bystander. Because many patients with NAFLD display features of the metabolic syndrome, other factors, such as dyslipidemia, hypertension and hyperglycemia, could account for the relationship between NAFLD and CVD. ${ }^{2}$ On the other hand, it has been suggested that NAFLD, in particular nonalcoholic steatohepatitis, is by itself linked to CVD by proinflammatory cytokines. $^{4}$

Genetic epidemiology may provide a solution to this conundrum. Because an individual is 'randomized' at birth to receive either a genetic variant that protects from, or predisposes to, a particular trait - in this case NAFLD - the association between this genetic variant and CVD will provide an answer as to whether or not that trait is causally involved in the pathophysiological process. Such a Mendelian randomization approach has already identified plasma lowdensity lipoprotein (LDL) cholesterol, triglycerides and (postprandial) glucose levels as causal factors, whereas the inflammatory factor C-reactive protein has been ruled out. ${ }^{5}$

To date, data on NAFLD genes in relation to CVD have been scarce. Previous studies on the transmembrane 6 superfamily member 2 gene (TM6SF2) have convincingly shown that variants in this gene predispose to all stages of NAFLD but protect from CVD. ${ }^{6-8}$ We recently reported a similar disentanglement for the patatin-like phospholipase domain containing 3 gene (PNPLA3) $^{9}$, the most replicated NAFLD gene. ${ }^{10,11}$

These findings question the presence of a causal, positive relationship between NAFLD/nonalcoholic steatohepatitis and CVD, although both PNPLA3 and TM6SF2 are also associated with lower secretion of VLDL particles ${ }^{8,12}$, which may account for the protection from CVD. 
In the current study, we sought to gain more insight into the causal relationship between NAFLD and coronary artery disease (CAD). For this, we clustered previously reported, common NAFLD genetic variants and tested for association with CAD. Given the complex relationship between NAFLD and dyslipidemia - which is a major cardiovascular risk factor by itself - we additionally studied the association of these variants with plasma lipids and their role in explaining the relationship with CAD.

\section{Methods}

\section{Selection of NAFLD susceptibility genes}

Selection of NAFLD susceptibility genes started with recent, concise overviews on this topic ${ }^{13-16}$ and was subsequently expanded by a Medline search for "NAFLD" and "gene". Because some genetic association studies have been conducted in relatively small populations and are therefore more likely to be underpowered, a stringent selection procedure was applied. An NAFLD gene was included in the present study when one of the following criteria was fulfilled: (1) The gene was identified by genome-wide association studies (GWASs) for NAFLD, as diagnosed by either histology or imaging; (2) the gene was identified by GWASs for an NAFLD-related trait - e.g., liver enzymes or alcoholic fatty liver disease (AFLD) - and subsequently confirmed in an NAFLD cohort; or (3) the gene was reported by multiple case-control studies in NAFLD cohorts and subsequently confirmed by a meta-analysis.

\section{Association of NAFLD susceptibility genes with CAD}

The association between the NAFLD genes and CAD was assessed under the assumption of an additive mode of inheritance in the Coronary Artery Disease Genome-wide Replication and Meta-analysis plus the Coronary Artery Disease (CARDIoGRAMplusC4D) Consortium cohort. This cohort contains 8.6 million genetic variants from 48 GWASs, either case-control studies ( 75\%) or (population-based) prospective cohort studies. The median number of participants per study was 2,874 (interquartile range: $1,557-5,291$ ). The overall dataset includes 60,801 cases with CAD and 123,504 controls of predominantly European and Asian ancestry. ${ }^{17}$ Although the definition of CAD differed to some extent between the studies that were part of the CARDIoGRAMplusC4D cohort, it generally included myocardial infarction, acute coronary syndrome, coronary artery bypass grafting, coronary angioplasty, (un)stable angina and/or coronary stenosis of $>50 \%$. 
Approximately $70 \%$ of the CAD cases had a reported history of myocardial infarction, which was generally defined as the combination of symptoms, typical abnormalities on electrocardiogram and elevation of cardiac enzymes. ${ }^{17}$ The association between NAFLD susceptibility genes and myocardial infarction was studied as a secondary outcome.

The summarized data on CAD and myocardial infarction were contributed by CARDIoGRAMplusC4D investigators and downloaded from www.cardiogramplusc4d.org (dataset "CARDloGRAMplusC4D 1000 Genomes-based GWAS").

\section{Association of NAFLD susceptibility genes with plasma lipids}

Because plasma lipid levels were not available in CARDloGRAMplusC4D, the relationships between the NAFLD-associated polymorphisms and plasma lipids, i.e., total cholesterol, LDL cholesterol, high-density lipoprotein (HDL) cholesterol and triglycerides, were studied by using the previously published joint Metabochip and GWAS data from the Global Lipids Genetics Consortium ( $n=188,577$ European-ancestry individuals).$^{18}$ In case a genetic variant was not present in this database, the analysis was conducted with a neighboring variant that is in strong linkage disequilibrium $\left(r^{2}>0.8\right)$.

\section{Statistical analyses}

Clustering of the NAFLD susceptibility genes to test for an association with CAD and myocardial infarction was done by constructing a weighted fixed effects model, which is similar to an inverse-variance weighted meta-analysis ${ }^{19}$ (see Supplementary Materials for more details). The effect sizes and variances of the individual genes were derived from the CARDIoGRAMplusC4D dataset. The final, weighted fixed effects model provides an estimate of the weighted average of the true effects in the set of genes. Because an additive model was used, this weighted average should be interpreted as the CAD risk conferred by one (average) NAFLD risk allele. To study the impact of genes that affect NAFLD risk by impairment of VLDL secretion - and thereby at the same time decrease plasma lipid levels - we additionally conducted a sensitivity analysis after exclusion of these genes.

Because NAFLD status was not present in CARDloGRAMplusC4D and previous genetic studies have assessed NAFLD by different methods (e.g., biopsy, computed tomography and ultrasound) and expressed NAFLD in different ways (e.g., dichotomous, ordinal and continuous), an analysis in which 
the gene-exposure relationship is regressed against the gene-outcome relationship was deemed unreliable.

Finally, the contribution of plasma lipids in explaining the observed heterogeneity was evaluated with meta-regression. All analyses were conducted with the R statistical software using the metafor package. ${ }^{19}$

\section{Results}

\section{Selection of NAFLD susceptibility genes}

The literature search resulted in 12 NAFLD susceptibility variants, of which 5 were selected based on previous GWASs for NAFLD (LYPLAL1, GCKR, PPP1R3B, PNPLA3 and TM6SF2) $)^{8,10,20}, 4$ from previous GWASs for NAFLDrelated traits combined with at least one case-control study in NAFLD (TRIB1, FADS1-2-3, ERLIN1-CHUK-CWF19L1 and MBOAT7) ${ }^{21-27}$ and 3 from casecontrol studies in NAFLD that were subsequently confirmed by a meta-analysis $(A D I P O Q, M T T P, P E M T)^{28-30}$ (Table 5.1). The tissue-expression and global function of the genes presumed to underlie these associations are listed in Supplementary Table S5.1. Only one, GCKR, is specifically expressed in the liver.

\section{Relationship between NAFLD genetic variants and CAD}

Clustering of all 12 NAFLD single-nucleotide polymorphisms (SNPs) did not result in an association with CAD (OR: 1.0; 95\% Cl: 0.99;1.00; $p=0.63$ ) (Figure 5.1). Similar results were obtained when myocardial infarction was used as the outcome (OR: 1.0; 95\% Cl: 0.99;1.01; p=0.93) (Supplementary Figure S5.1). Furthermore, results were not essentially different when only the most validated NAFLD genes, i.e., PNPLA3, GCKR, TM6SF2 and MBOAT7, were used for analysis (Supplementary Figure S5.2). Additional sensitivity analyses revealed a positive association with CAD (OR: 1.01; 95\% Cl: 1.00;1.02; $p=0.02$ ) after exclusion of those SNPs that affect NAFLD through impaired VLDL secretion, i.e., PEMT, MTTP, PNPLA3 and TM6SF2 (Supplementary Table S5.1; Supplementary Figure S5.3). As expected, clustering of the four excluded genes resulted in a negative relationship with CAD (OR: $0.97 ; 95 \% \mathrm{Cl}$ : 0.96;0.99; $p<0.0001$ ) (Supplementary Figure S5.3). 


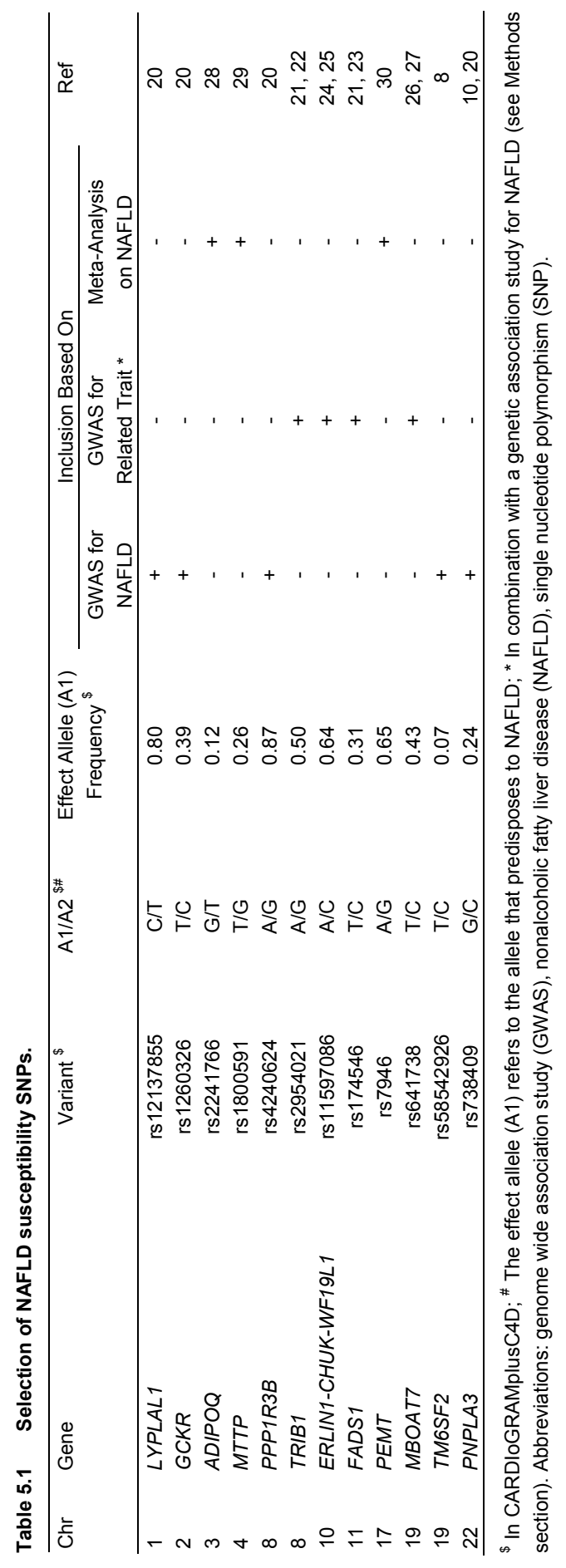




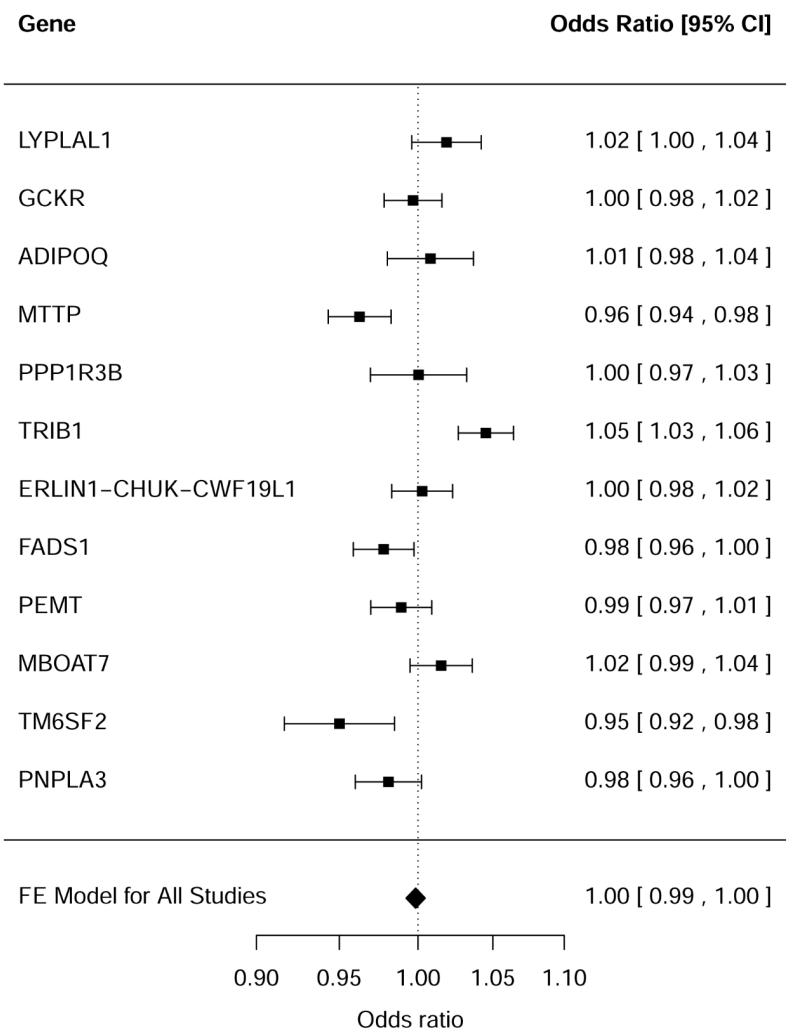

Figure 5.1 Relationship between NAFLD susceptibility genes and CAD.

Relationship between NAFLD genetic variants and plasma lipids

The association of the individual NAFLD SNPs with plasma lipids was subsequently assessed to evaluate the impact of plasma lipids on the observed relationships with CAD. Results for total cholesterol, LDL cholesterol, HDL cholesterol and triglyceride levels were obtained from the Global Lipids Genetics Consortium and listed in Table 5.2. The effects of the individual NAFLD SNPs on plasma lipids were subsequently plotted (on the $x$ axis) versus their effects on CAD risk (on the $y$ axis) (Figure 5.2). A strong correlation was observed for both total cholesterol and LDL cholesterol with CAD ( $r=0.73$ and $p=0.008 ; r=0.76$ and $p=0.004$ ) (Figure 5.2, panels $A$ and $B$, respectively) but not for plasma triglycerides and $\mathrm{HDL}$ cholesterol $(r=0.57$ and $p=0.05 ; r=-0.14$ and $p=0.66$ ) (Figure 5.2, panels $C$ and $D$, respectively). Similar results were observed when myocardial infarction was used as an outcome (Supplementary Figure S5.4). 


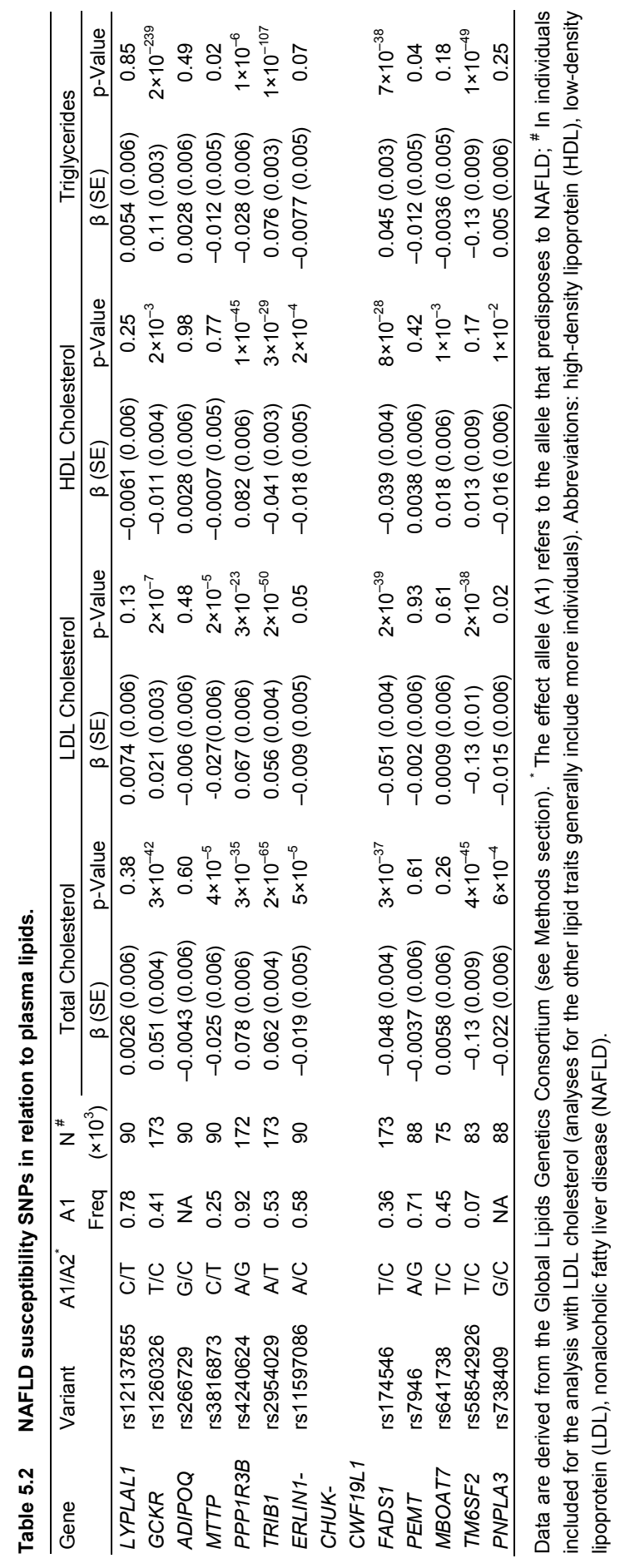


When the original analysis (Figure 5.1) was repeated after adjustment for LDL cholesterol, the degree of heterogeneity was indeed reduced, albeit not completely (from $Q=58.4$ and $p<0.0001$ in the primary analysis (Figure 5.1 ) to $\mathrm{Q}=26.9$ and $p=0.005$ after correction for LDL cholesterol).
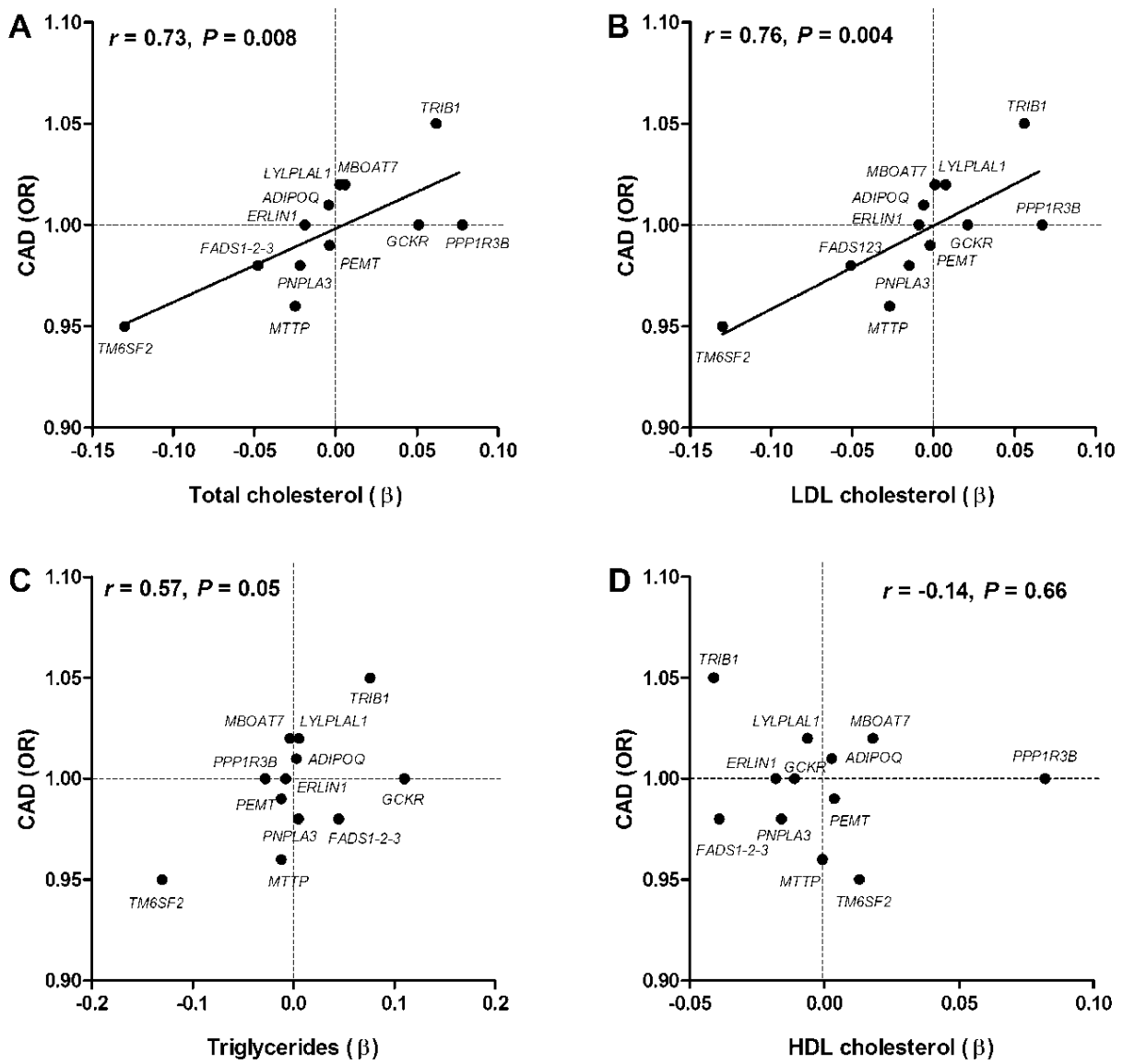

Figure 5.2 Relationship between (A) total cholesterol, (B) LDL cholesterol, (C) triglycerides and (D) HDL cholesterol and CAD conferred by NAFLD susceptibility genes.

\section{Discussion}

The Mendelian randomization approach has demonstrated its utility in identifying causal factors in the pathogenesis of CAD, not only for plasma biomarkers (e.g., LDL cholesterol) but also for traits such as body mass index 
(BMI) and waist-to-hip ratio. $^{31}$ The current study shows that NAFLD susceptibility genes do not cause CAD per se.

One of the key assumptions of the Mendelian randomization approach is that the genetic variants that affect the exposure of interest do not exert pleiotropic effects. The risk of pleiotropy increases when the exposure of interest is a complex, non-protein phenotype ${ }^{5}$, as is the case for NAFLD. Given the central role the liver plays in (systemic) metabolic processes, it is difficult to identify genes that only affect NAFLD risk. In this respect, a distinction should be made between vertical and horizontal pleiotropy (Supplementary Figure 5.5). Pleiotropy is vertical when the genetic variant is associated with a trait or biomarker that is downstream in the pathway that relates the exposure of interest with the clinical endpoint (CAD). Because this is a mediating effect, it does not invalidate the Mendelian randomization assumptions. In contrast, pleiotropy is horizontal when the genetic variant affects pathways other than the exposure of interest. ${ }^{31}$ The complex, intertwined relationship between NAFLD genes and plasma lipids probably results in both horizontal and vertical pleiotropy in the present study. Stable isotope studies have demonstrated that an increased flux of fatty acids and de novo lipogenesis contribute to intrahepatic lipid accumulation and, consequently, drive VLDL production. ${ }^{32}$ The observed associations of SNPs affecting fatty acid flux and de novo lipogenesis with plasma lipids (e.g., TRIB1 and GCKR) are therefore likely downstream effects of NAFLD and, hence, an example of vertical pleiotropy. Those SNPs that predispose to NAFLD through impaired VLDL secretion (i.e., MTTP, PEMT, PNPLA3 and TM6SF2) simultaneously decrease plasma lipids. Because NAFLD does in this case not precede the effect on plasma lipids, it could be viewed as an example of horizontal pleiotropy (Supplementary Figure S5.5). Of interest, when we conducted a sensitivity analysis without the latter genes, a positive relationship with CAD was observed.

Exclusion of those SNPs that affect NAFLD through impaired VLDL secretion does not necessarily eliminate all horizontal pleiotropy. Many SNPs were not specifically expressed in the liver (Supplementary Table S5.1). Furthermore, the neutral effect of one liver-specific gene (GCKR) on CAD may be explained by reported protective effects on kidney function. ${ }^{33}$ The use of a polygenic risk score, as done in the present study, may 'balance out' horizontal pleiotropy. ${ }^{31}$ This can be formally tested by using MR-Egger regression or a weighted median method. Both methods require both gene-exposure and gene-outcome data. $^{34,35}$ Unfortunately, gene-exposure data were not available for reasons outlined in the Methods section. This should be regarded as a limitation of this study. 
The putative mechanism linking NAFLD to CAD has been a topic of interest in previous comprehensive reviews. Although low-grade inflammation has often been suggested as the principal mediator ${ }^{4,36}$, the present study strongly suggests that plasma lipids are more important. Many of the NAFLD genes that were included in the present study not only predispose to intrahepatic lipid accumulation but also to steatohepatitis. ${ }^{8,10,20,22,26,37}$ Nevertheless, there was a strong relationship between plasma lipids and CAD, as conferred by the 12 NAFLD susceptibility SNPs, which is very much in line with a previous Mendelian randomization study in which 185 lipid susceptibility variants from these same cohorts were used to examine the causal relation between plasma lipids and $C A D .{ }^{38}$ Moreover, the intercepts of these relationships included zero (Figure 5.2), which implicates that when the NAFLD susceptibility SNPs do not have any effect on plasma lipids there is also no effect on CAD. This suggests that other vertical pleiotropic effects (such as low-grade inflammation) or unbalanced horizontal pleiotropy do not play a major role in our analyses, as they would have resulted in an intercept deviating from zero. Unfortunately, we did not dispose of a set of inflammatory markers to formally investigate the mediating role of inflammation on the relationship between NAFLD and CAD.

The current observations have important implications for future therapies that target NAFLD. Genetic studies have been instructive in predicting pharmacological effects. An illustrative example is proprotein convertase subtilisin kexin type 9; studies demonstrating that lipid-lowering variants in the proprotein convertase subtilisin kexin type 9 gene protected from CAD preceded the recently reported results from phase 3 clinical trials. ${ }^{39-42}$ The strong relationship between plasma lipids and CAD risk conferred by the NAFLD susceptibility variants strongly suggests that the design of an antiNAFLD drug should take into account any effects on plasma lipids. Otherwise, the beneficial effects on NAFLD may be offset by an increased risk to develop CAD. Genes in the right upper quadrant (Figure 5.1, panel A-C) therefore appear to be the best therapeutic candidates.

This study has some strengths and limitations besides the previously mentioned issues regarding (horizontal) pleiotropy. The CARDIoGRAMplusC4D dataset with more than 180,000 individuals genotyped for common and low-frequency variants allowed us to study the relationship between previously reported NAFLD susceptibility genes and CAD with sufficient statistical power. Because this dataset contains summarized data, it was not possible to perform subgroup analyses, stratified for sex or ethnicity. Common genetic variants generally have small effect sizes ${ }^{43}$, which probably explains the modest CAD risks conferred by the NAFLD genes. Of interest, 
genetic variants in TM6SF2 have been shown to confer a large effect on NAFLD, plasma lipids and CAD risk. ${ }^{6}$ In contrast, previous studies have reported a large effect size for the association between PNPLA3 and NAFLD ${ }^{44}$, whereas a small effect was observed for the association with plasma lipids and CAD in the current study. These data corroborate the suggestion that the effect size of NAFLD genetic variants on CAD risk is related to their effects on plasma lipids and not on liver disease per se, which was indeed confirmed in a recent Mendelian randomization study in which PNPLA3 was used as an instrument. ${ }^{45}$ PNPLA3 and TM6SF2, as well as MBOAT7, have not only been associated with NAFLD but also with other liver diseases, such as AFLD. ${ }^{27}$ Although NAFLD is currently the most common liver disease in Western society ${ }^{46}$, it is likely that patients with AFLD have also been included in CARDIoGRAMplusC4D. The conclusions drawn in the present study are therefore probably not confined to NAFLD, but to intrahepatic lipid accumulation in general.

Unfortunately, data on plasma lipids or other potential mediators/confounders of the relationship between NAFLD and CAD, such as type 2 diabetes, were not present in the CARDIoGRAMplusC4D dataset. Previous GWASs have shown that variants in PNPLA3 and TM6SF2 are also associated with type 2 diabetes. ${ }^{47}$ We tried to resolve this, at least for plasma lipids, by using data from the well-recognized Global Lipids Genetics Consortium, in line with previous reports. ${ }^{38}$ Although, the consequential strong relationship between plasma lipids and CAD, as conferred by the NAFLD susceptibility genes (Figure 5.2), suggests an active role of plasma lipids, the lack of individual lipid data in CARDIoGRAMplusC4D limits strong inferences on the role of plasma lipids as a mediator of the relationship between NAFLD and CAD.

We applied stringent selection criteria for the NAFLD susceptibility genes, which makes the inclusion of false-positive NAFLD variants unlikely. A disadvantage is that the number of genes included in the analyses was relatively low, which may have reduced statistical power. Finally, these genetic variants can be in linkage disequilibrium with a variant in another gene, which is actually responsible for the observed association.

In summary, the present study nuances previous epidemiological studies by demonstrating that NAFLD susceptibility genes do not unequivocally cause CAD. Further studies that focus on the mediating effects of plasma lipids are warranted, as plasma lipids accounted for a substantial degree of the observed heterogeneity. These observations have important implications for future therapeutic strategies against NAFLD. 


\section{Literature}

1. Charlton MR, Burns JM, Pedersen RA, Watt KD, Heimbach JK, Dierkhising RA. Frequency and outcomes of liver transplantation for nonalcoholic steatohepatitis in the United States. Gastroenterology 2011;141:1249-53.

2. Ong JP, Pitts A, Younossi ZM. Increased overall mortality and liver-related mortality in nonalcoholic fatty liver disease. J Hepatol 2008;49:608-12.

3. Targher G, Byrne CD, Lonardo A, Zoppini G, Barbui C. Non-alcoholic fatty liver disease and risk of incident cardiovascular disease: A meta-analysis. J Hepatol 2016;65:589-600.

4. Adams LA, Anstee QM, Tilg H, Targher G. Non-alcoholic fatty liver disease and its relationship with cardiovascular disease and other extrahepatic diseases. Gut 2017;66: 1138-53.

5. Jansen $\mathrm{H}$, Samani NJ, Schunkert $\mathrm{H}$. Mendelian randomization studies in coronary artery disease. Eur Heart J 2014;35:1917-24.

6. Dongiovanni $\mathrm{P}$, Petta S, Maglio $\mathrm{C}$, et al. Transmembrane 6 superfamily member 2 gene variant disentangles nonalcoholic steatohepatitis from cardiovascular disease. Hepatology 2015;61:506-14.

7. Holmen $\mathrm{OL}$, Zhang $\mathrm{H}$, Fan $\mathrm{Y}$, et al. Systematic evaluation of coding variation identifies a candidate causal variant in TM6SF2 influencing total cholesterol and myocardial infarction risk. Nat Genet 2014;46:345-51.

8. Kozlitina J, Smagris E, Stender S, et al. Exome-wide association study identifies a TM6SF2 variant that confers susceptibility to nonalcoholic fatty liver disease. Nat Genet 2014;46: 352-6.

9. Simons N, Isaacs A, Koek GH, Kuc S, Schaper NC, Brouwers M. PNPLA3, TM6SF2, and MBOAT7 Genotypes and Coronary Artery Disease. Gastroenterology 2017;152:912-3.

10. Romeo S, Kozlitina J, Xing C, et al. Genetic variation in PNPLA3 confers susceptibility to nonalcoholic fatty liver disease. Nat Genet 2008;40:1461-5.

11. Sookoian S, Pirola CJ. Meta-analysis of the influence of I148M variant of patatin-like phospholipase domain containing 3 gene (PNPLA3) on the susceptibility and histological severity of nonalcoholic fatty liver disease. Hepatology 2011;53:1883-94.

12. Pirazzi C, Adiels M, Burza MA, et al. Patatin-like phospholipase domain-containing 3 (PNPLA3) I148M (rs738409) affects hepatic VLDL secretion in humans and in vitro. J Hepatol 2012;57:1276-82.

13. Dongiovanni P, Valenti L. Genetics of nonalcoholic fatty liver disease. Metabolism 2016;65:1026-37.

14. Kahali B, Halligan B, Speliotes EK. Insights from Genome-Wide Association Analyses of Nonalcoholic Fatty Liver Disease. Semin Liver Dis 2015;35:375-91.

15. Macaluso FS, Maida M, Petta S. Genetic background in nonalcoholic fatty liver disease: A comprehensive review. World J Gastroenterol 2015;21:11088-111.

16. Anstee QM, Day CP. The Genetics of Nonalcoholic Fatty Liver Disease: Spotlight on PNPLA3 and TM6SF2. Semin Liver Dis 2015;35:270-90.

17. Nikpay M, Goel A, Won HH, et al. A comprehensive 1,000 Genomes-based genome-wide association meta-analysis of coronary artery disease. Nat Genet 2015;47:1121-30.

18. Willer CJ, Schmidt EM, Sengupta S, et al. Discovery and refinement of loci associated with lipid levels. Nat Genet 2013;45:1274-83.

19. Viechtbauer W. Conducting Meta-Analyses in $\mathrm{R}$ with the metafor Package. Journal of Statistical Software;36.

20. Speliotes EK, Yerges-Armstrong LM, Wu J, et al. Genome-wide association analysis identifies variants associated with nonalcoholic fatty liver disease that have distinct effects on metabolic traits. PLoS Genet 2011;7:e1001324.

21. Chambers JC, Zhang W, Sehmi J, et al. Genome-wide association study identifies loci influencing concentrations of liver enzymes in plasma. Nat Genet 2011;43:1131-8. 
22. Kitamoto A, Kitamoto T, Nakamura $\mathrm{T}$, et al. Association of polymorphisms in GCKR and TRIB1 with nonalcoholic fatty liver disease and metabolic syndrome traits. Endocr $J$ 2014;61:683-9.

23. Wang L, Athinarayanan S, Jiang G, Chalasani N, Zhang M, Liu W. Fatty acid desaturase 1 gene polymorphisms control human hepatic lipid composition. Hepatology 2015;61:119-28.

24. Yuan X, Waterworth D, Perry JR, et al. Population-based genome-wide association studies reveal six loci influencing plasma levels of liver enzymes. Am J Hum Genet 2008;83:520-8.

25. Feitosa MF, Wojczynski MK, North KE, et al. The ERLIN1-CHUK-CWF19L1 gene cluster influences liver fat deposition and hepatic inflammation in the NHLBI Family Heart Study. Atherosclerosis 2013;228:175-80.

26. Mancina RM, Dongiovanni P, Petta S, et al. The MBOAT7-TMC4 Variant rs641738 Increases Risk of Nonalcoholic Fatty Liver Disease in Individuals of European Descent. Gastroenterology 2016;150:1219-30.e6.

27. Buch S, Stickel F, Trepo E, et al. A genome-wide association study confirms PNPLA3 and identifies TM6SF2 and MBOAT7 as risk loci for alcohol-related cirrhosis. Nat Genet 2015;47:1443-8.

28. Wang J, Guo XF, Yu SJ, et al. Adiponectin polymorphisms and non-alcoholic fatty liver disease risk: a meta-analysis. J Gastroenterol Hepatol 2014;29:1396-405.

29. Zheng $\mathrm{W}$, Wang L, Su X, Hu XF. MTP $-493 \mathrm{G}>\mathrm{T}$ polymorphism and susceptibility to nonalcoholic fatty liver disease: a meta-analysis. DNA Cell Biol 2014;33:361-9.

30. Tan HL, Mohamed R, Mohamed Z, Zain SM. Phosphatidylethanolamine N-methyltransferase gene rs7946 polymorphism plays a role in risk of nonalcoholic fatty liver disease: evidence from meta-analysis. Pharmacogenet Genomics 2016;26:88-95.

31. Dale CE, Fatemifar G, Palmer TM, et al. Causal Associations of Adiposity and Body Fat Distribution With Coronary Heart Disease, Stroke Subtypes, and Type 2 Diabetes Mellitus: A Mendelian Randomization Analysis. Circulation 2017;135:2373-88.

32. Fabbrini E, Mohammed BS, Magkos F, Korenblat KM, Patterson BW, Klein S. Alterations in adipose tissue and hepatic lipid kinetics in obese men and women with nonalcoholic fatty liver disease. Gastroenterology 2008;134:424-31.

33. Kottgen A, Pattaro C, Boger CA, et al. New loci associated with kidney function and chronic kidney disease. Nat Genet 2010;42:376-84.

34. Bowden J, Davey Smith G, Haycock PC, Burgess S. Consistent Estimation in Mendelian Randomization with Some Invalid Instruments Using a Weighted Median Estimator. Genet Epidemiol 2016;40:304-14.

35. Bowden J, Davey Smith G, Burgess S. Mendelian randomization with invalid instruments: effect estimation and bias detection through Egger regression. Int J Epidemiol 2015;44: $512-25$.

36. Targher G, Day CP, Bonora E. Risk of cardiovascular disease in patients with nonalcoholic fatty liver disease. N Engl J Med 2010;363:1341-50.

37. Gambino R, Cassader M, Pagano G, Durazzo M, Musso G. Polymorphism in microsomal triglyceride transfer protein: a link between liver disease and atherogenic postprandial lipid profile in NASH? Hepatology 2007;45:1097-107.

38. Do R, Willer CJ, Schmidt EM, et al. Common variants associated with plasma triglycerides and risk for coronary artery disease. Nat Genet 2013;45:1345-52.

39. Kathiresan S. A PCSK9 missense variant associated with a reduced risk of early-onset myocardial infarction. N Engl J Med 2008;358:2299-300.

40. Cohen JC, Boerwinkle E, Mosley TH, Jr., Hobbs HH. Sequence variations in PCSK9, low LDL, and protection against coronary heart disease. N Engl J Med 2006;354:1264-72.

41. Sabatine MS, Giugliano RP, Wiviott SD, et al. Efficacy and safety of evolocumab in reducing lipids and cardiovascular events. N Engl J Med 2015;372:1500-9.

42. Robinson JG, Farnier M, Krempf M, et al. Efficacy and safety of alirocumab in reducing lipids and cardiovascular events. N Engl J Med 2015;372:1489-99. 
43. Manolio TA, Collins FS, Cox NJ, et al. Finding the missing heritability of complex diseases. Nature 2009;461:747-53.

44. Valenti L, Dongiovanni P, Ginanni Corradini S, Burza MA, Romeo S. PNPLA3 I148M variant and hepatocellular carcinoma: a common genetic variant for a rare disease. Dig Liver Dis 2013;45:619-24.

45. Lauridsen BK, Stender S, Kristensen TS, et al. Liver fat content, non-alcoholic fatty liver disease, and ischaemic heart disease: Mendelian randomization and meta-analysis of 279 013 individuals. Eur Heart J 2018;39:385-93.

46. Younossi ZM, Stepanova M, Afendy M, et al. Changes in the prevalence of the most common causes of chronic liver diseases in the United States from 1988 to 2008. Clin Gastroenterol Hepatol 2011;9:524-30.e1; quiz e60.

47. Liu DJ, Peloso GM, Yu H, et al. Exome-wide association study of plasma lipids in $>300,000$ individuals. Nat Genet 2017;49:1758-66. 


\section{Supplementary Materials}

Supplementary Information 1 - Statistical analyses, clustering of NAFLD susceptibility genes

Clustering of the NAFLD susceptibility genes to test for an association with CAD was done by constructing a fixed effects model (weighted model fitting), which is commonly used in meta-analyses. For a set of $i=1, \ldots . . \mathrm{k}$ independent genes, let $y_{i}$ denote the observed value of the effect size, i.e., CAD risk, in the ith gene.

Let $\theta_{i}$ denote the corresponding (unknown) true effect or outcome, such that

$y_{i} \mid \theta_{i} \sim N\left(\theta_{i}, v_{i}\right)$

where $y_{i}$ and $v_{i}$ (=sampling variance) are derived from the CARDioGRAMplusC4D dataset. The weighted fixed effects model provides an estimate of

$\overline{\theta_{w}}=\sum_{i=1}^{k} w_{i} \theta_{i} / \sum_{i=1}^{k} w_{i}$

that is, the weighted average of the true effects/outcomes in the set of $k$ genes, with weights equal to $w_{i}=1 / v_{i}$ (often described as the inverse-variance method in the meta-analytic literature $\left.{ }^{1}\right)$. This type of analysis is very similar to the inverse-variance weighted combination of ratio estimates that is used in classical Mendelian randomization analysis with multiple genetic variants using summarized data. ${ }^{2}$ 


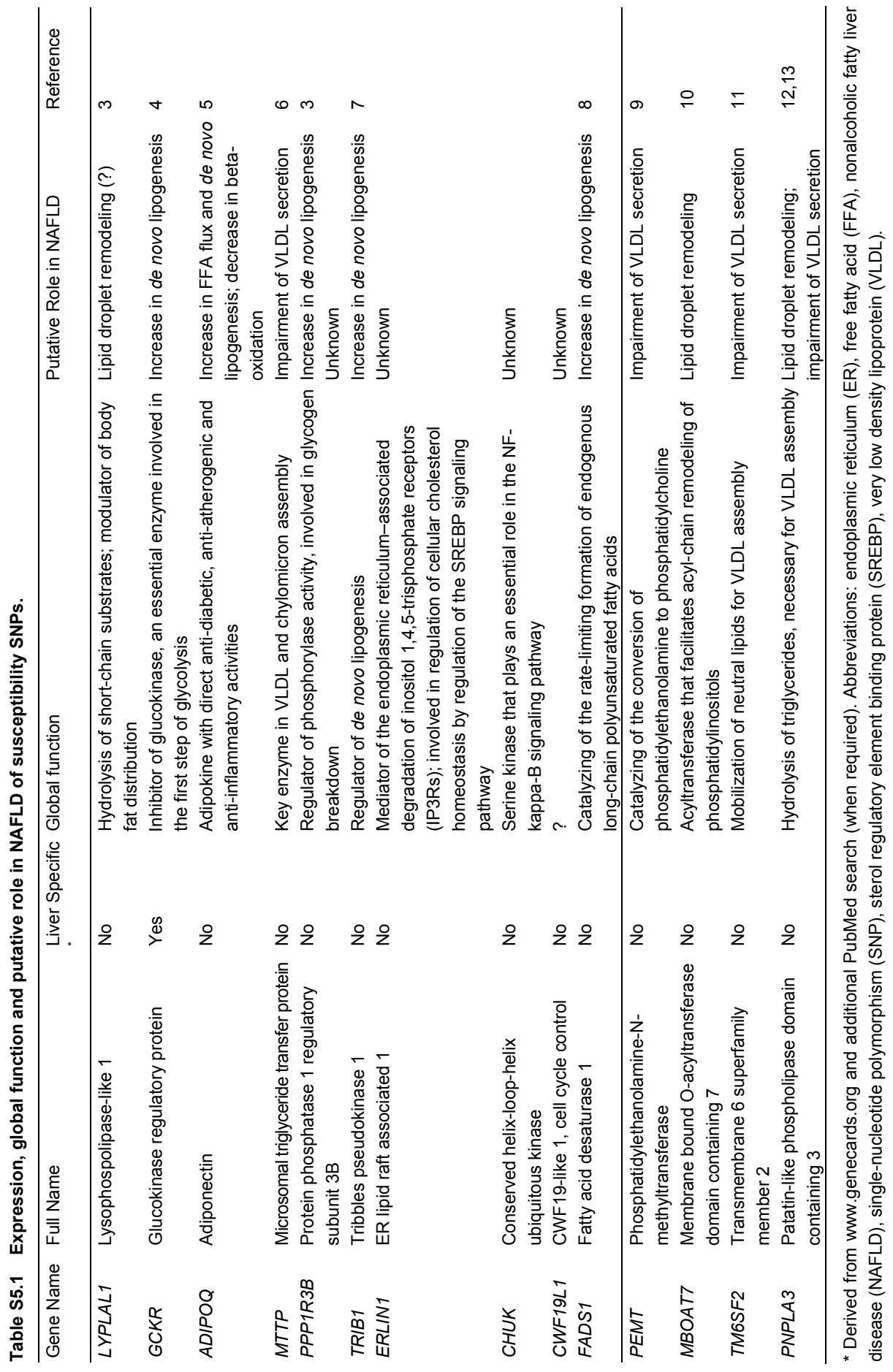


Odds Ratio $[95 \% \mathrm{Cl}]$

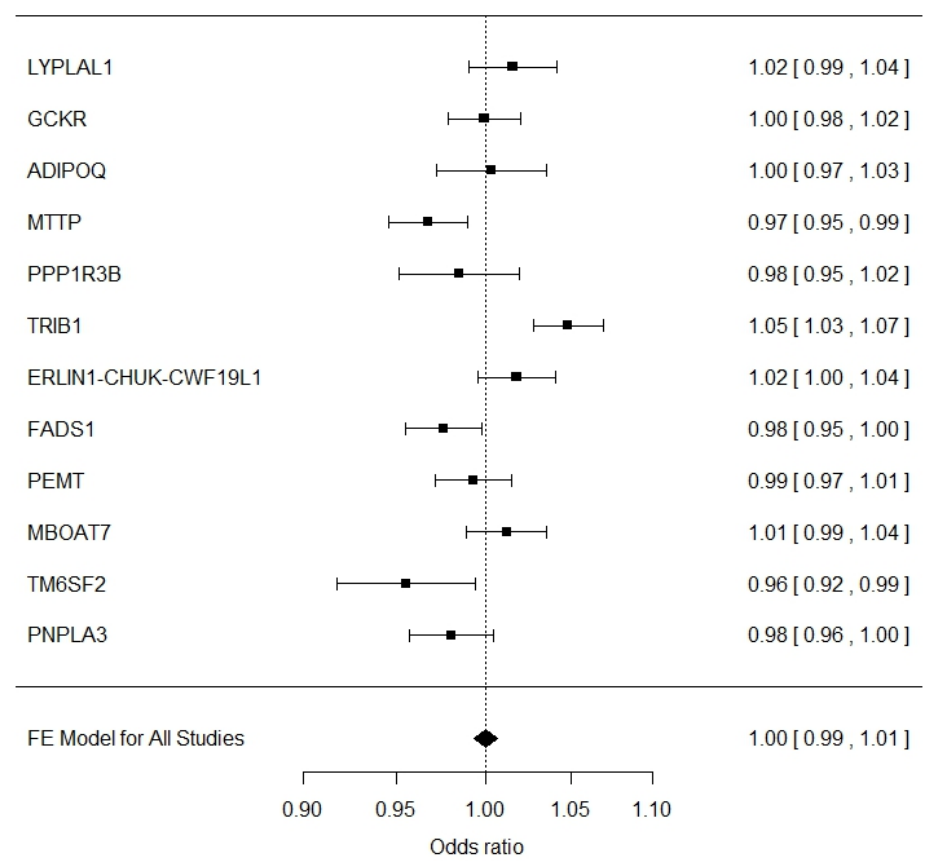

Figure S5.1 Relationship between NAFLD susceptibility genes and myocardial infarction. Abbreviations: confidence interval $(\mathrm{Cl})$, fixed effects (FE), nonalcoholic fatty liver disease (NAFLD).

Gene

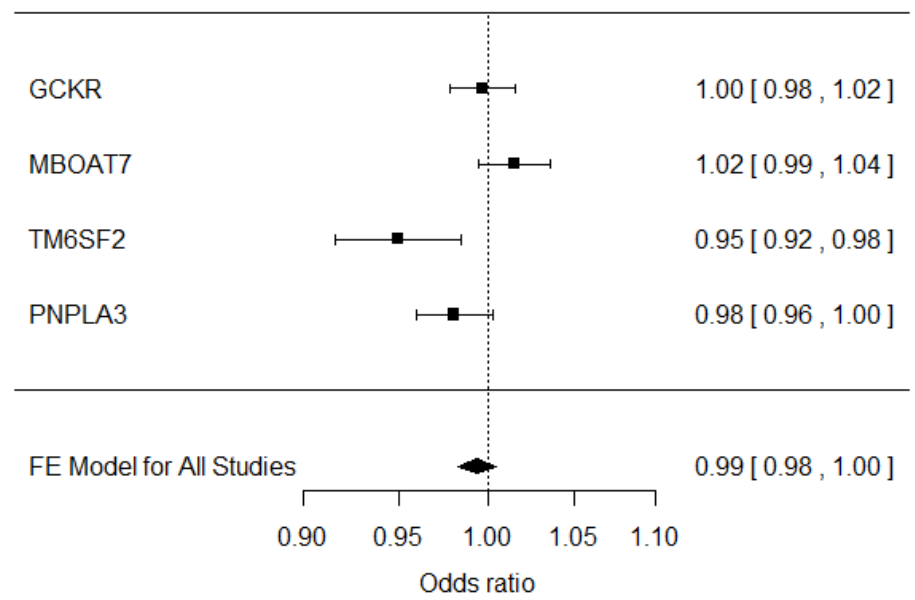

Figure S5.2 Most validated NAFLD susceptibility genes in relation to CAD. Abbreviations: coronary artery disease (CAD), confidence interval (Cl), fixed effects (FE), nonalcoholic fatty liver disease (NAFLD). 


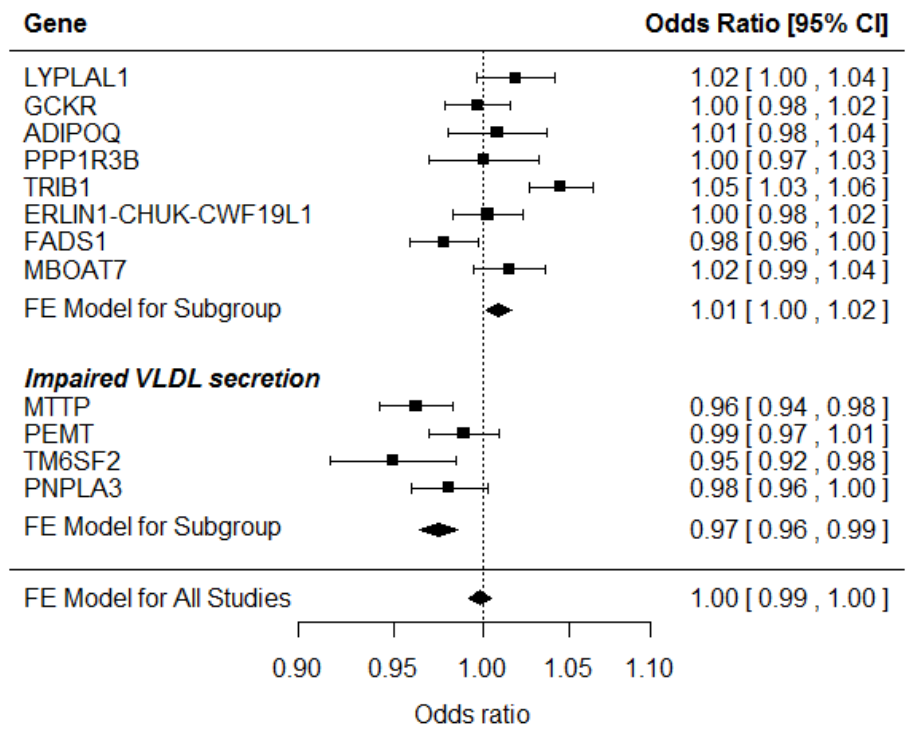

Figure S5.3 NAFLD susceptibility genes (excluding genes involved in VLDL secretion pathway) in relation to CAD. Abbreviations: coronary artery disease (CAD), confidence interval $(\mathrm{Cl})$, fixed effects (FE), nonalcoholic fatty liver disease (NAFLD), very-low-density lipoprotein (VLDL). 

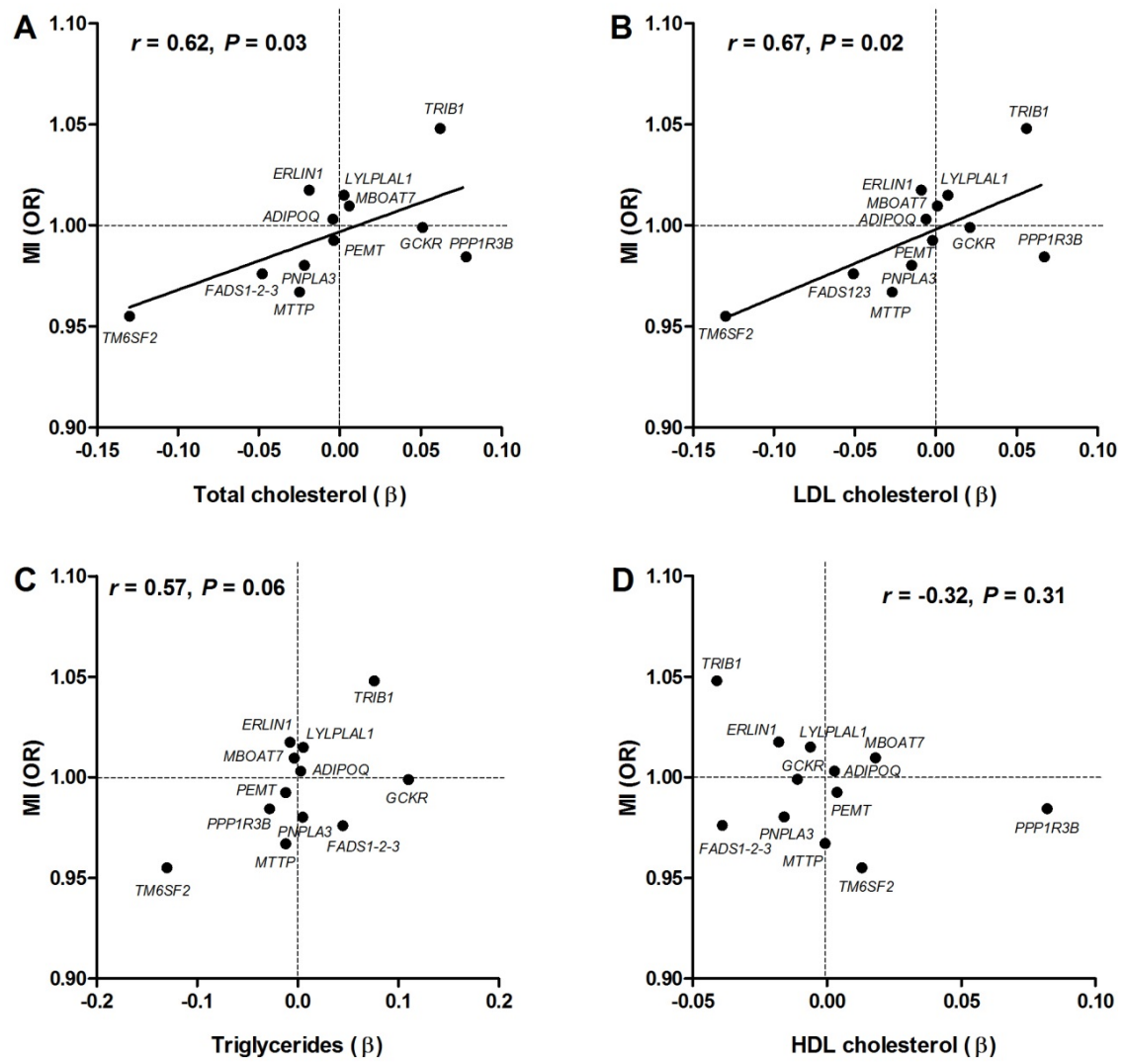

Figure S5.4 Relationship between total cholesterol (A), LDL cholesterol (B), triglycerides (C) and HDL cholesterol (D) and myocardial infarction conferred by NAFLD susceptibility genes. Abbreviations: high-density lipoprotein (HDL), low-density lipoprotein (LDL), myocardial infarction (MI), nonalcoholic fatty liver disease (NAFLD), odds ratio (OR). 


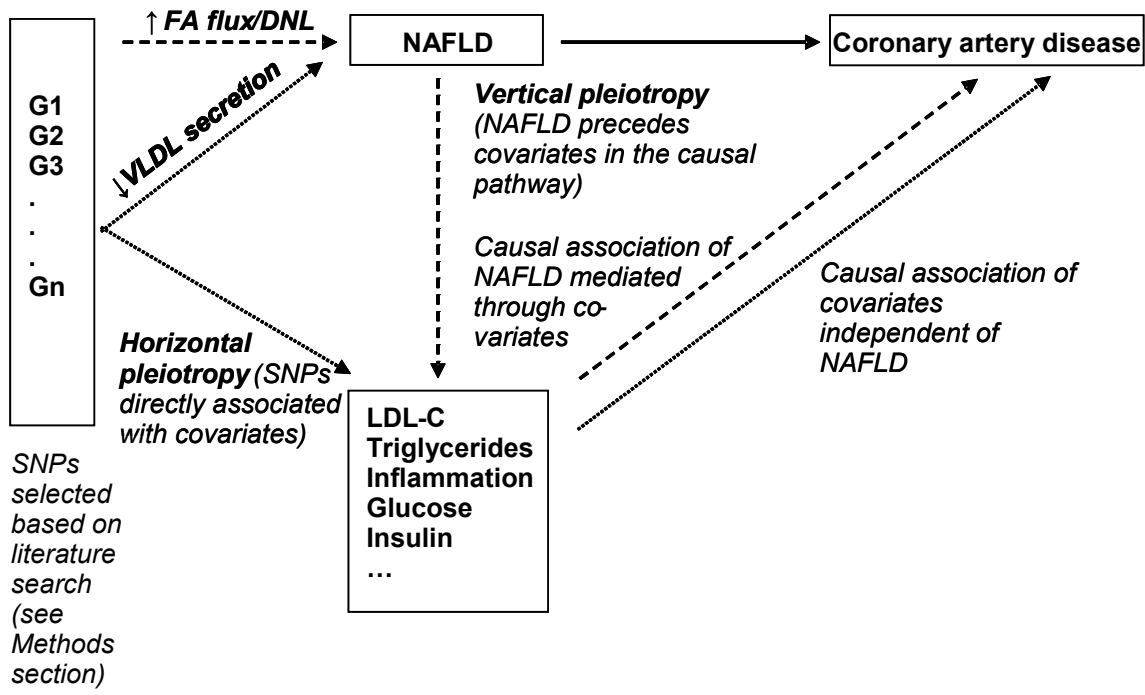

Figure S5.5 Influence of pleiotropy on the relationship between the NAFLD susceptibility genes and CAD. SNPs can predispose to the development of NAFLD through different mechanisms, i.e., increased FA flux, increased DNL (and reciprocal inhibition of beta-oxidation) and impaired VLDL secretion. Theoretically, NAFLD may have a direct effect on CAD risk (straight line). Alternatively, the association of an SNP with CAD can be mediated by downstream effects of NAFLD (vertical pleiotropy, dashed lines). It is also possible that an SNP does not only affect NAFLD but also covariates independent of NAFLD (horizontal pleiotropy, dotted lines). SNPs that predispose to NAFLD through impaired VLDL secretion at the same time decrease plasma lipids, which should be viewed as horizontal pleiotropy. Adapted from Dale et al. ${ }^{14}$ Abbreviations: coronary artery disease (CAD), de novo lipogenesis ( $D N L)$, fatty acid (FA), low-density lipoprotein (LDL), nonalcoholic fatty liver disease (NAFLD), single-nucleotide polymorphism (SNP). 


\section{Literature}

1. Viechtbauer W. Conducting Meta-Analyses in $\mathrm{R}$ with the metafor Package. Journal of Statistical Software;36.

2. Burgess S, Butterworth A, Thompson SG. Mendelian randomization analysis with multiple genetic variants using summarized data. Genet Epidemiol 2013;37:658-65.

3. Kahali B, Halligan B, Speliotes EK. Insights from Genome-Wide Association Analyses of Nonalcoholic Fatty Liver Disease. Semin Liver Dis 2015;35:375-91.

4. Santoro N, Caprio S, Pierpont B, Van Name M, Savoye M, Parks EJ. Hepatic De Novo Lipogenesis in Obese Youth Is Modulated by a Common Variant in the GCKR Gene. The J Clin Endocrinol Metab 2015;100:E1125-32.

5. Xu A, Wang Y, Keshaw H, Xu LY, Lam KS, Cooper GJ. The fat-derived hormone adiponectin alleviates alcoholic and nonalcoholic fatty liver diseases in mice. J Clin Invest 2003;112: 91-100.

6. Karpe F, Lundahl B, Ehrenborg E, Eriksson P, Hamsten A. A common functional polymorphism in the promoter region of the microsomal triglyceride transfer protein gene influences plasma LDL levels. Arterioscler Thromb Vasc Biol 1998;18:756-61.

7. Bauer RC, Sasaki M, Cohen DM, et al. Tribbles-1 regulates hepatic lipogenesis through posttranscriptional regulation of C/EBPalpha. J Clin Invest 2015;125:3809-18.

8. Davidson $\mathrm{MH}$. Omega-3 fatty acids: new insights into the pharmacology and biology of docosahexaenoic acid, docosapentaenoic acid, and eicosapentaenoic acid. Curr Opin Lipidol 2013;24:467-74.

9. Vance DE, Li Z, Jacobs RL. Hepatic phosphatidylethanolamine N-methyltransferase, unexpected roles in animal biochemistry and physiology. J Biol Chem 2007;282:33237-41.

10. Mancina RM, Dongiovanni P, Petta S, et al. The MBOAT7-TMC4 Variant rs641738 Increases Risk of Nonalcoholic Fatty Liver Disease in Individuals of European Descent. Gastroenterology 2016;150:1219-30.e6.

11. Kozlitina J, Smagris E, Stender S, et al. Exome-wide association study identifies a TM6SF2 variant that confers susceptibility to nonalcoholic fatty liver disease. Nat Genet 2014;46: 352-6.

12. Pirazzi C, Adiels M, Burza MA, et al. Patatin-like phospholipase domain-containing 3 (PNPLA3) I148M (rs738409) affects hepatic VLDL secretion in humans and in vitro. J Hepatol 2012;57:1276-82.

13. Ruhanen $\mathrm{H}$, Perttila J, Holtta-Vuori M, et al. PNPLA3 mediates hepatocyte triacylglycerol remodeling. J Lipid Res 2014;55:739-46.

14. Dale CE, Fatemifar G, Palmer TM, et al. Causal Associations of Adiposity and Body Fat Distribution With Coronary Heart Disease, Stroke Subtypes, and Type 2 Diabetes Mellitus: A Mendelian Randomization Analysis. Circulation 2017;135:2373-88. 
The relationship between nonalcoholic fatty liver disease and biomarkers of endothelial function 



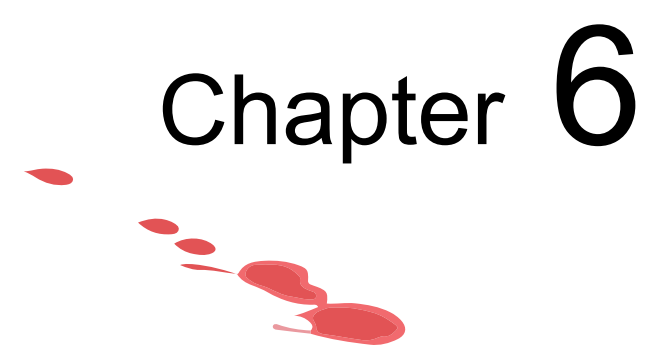

\section{Soluble E-selectin as a biomarker of hepatic endothelial function}
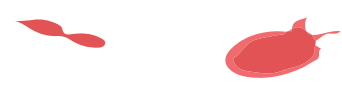

The endothelial function biomarker soluble E-selectin is associated with nonalcoholic fatty liver disease

Nynke Simons, Mitchell Bijnen, Kristiaan A.M. Wouters, Sander S. Rensen, Joline W.J. Beutens, Marleen M.J. van Greevenbroek, Leen M. 't Hart, Jan Willem M. Greve, Carla J.H. van der Kallen, Nicolaas C. Schaper, Casper G. Schalkwijk, Coen D.A. Stehouwer, Martijn C.G.J. Brouwers 


\section{Abstract}

\section{Background}

Plasma soluble E-selectin (sE-selectin) is a frequently used biomarker of systemic endothelial dysfunction. The present study explored the relationship between nonalcoholic fatty liver disease (NAFLD) and plasma sE-selectin levels.

\section{Methods}

Expression of E-selectin in liver, visceral adipose tissue (VAT) and muscle was studied in relation to plasma sE-selectin in severely obese individuals $(n=74)$. The course of hepatic E-selectin expression in relation to hepatic steatosis and inflammation was examined in C57BL/6 $\mathrm{LDLR}^{-/}$mice on a Western-type diet. The relationship between biomarkers of NAFLD, i.e., plasma aminotransferase (ALT) and NAFLD susceptibility genes (rs738409 [PNPLA3] and rs1260326 [GCKR]), and plasma sE-selectin was studied in the combined CODAM $(n=571)$ and Hoorn $(n=694)$ studies.

\section{Results}

E-selectin expression in liver, not VAT or muscle, was associated with plasma sE-selectin in severely obese individuals $(\beta=0.26 ; 95 \% \mathrm{Cl}: 0.05 ; 0.47)$. NAFLD severity was associated with hepatic E-selectin expression $(p=0.02)$ and plasma sE-selectin $(p=0.003)$. LDLR ${ }^{-/}$mice on a Western-type diet displayed increased hepatic E-selectin expression that followed the same course as hepatic inflammation, but not steatosis. In the CODAM study, plasma ALT was associated with plasma sE-selectin, independent of potential confounders $(\beta=0.25 ; 95 \% \mathrm{Cl}: 0.16 ; 0.34)$. Both rs738409 and rs1260326 were associated with higher plasma sE-selectin in the combined CODAM and Hoorn studies ( $p=0.01$ and $p=0.004$, respectively).

\section{Conclusions}

NAFLD and related markers are associated with higher expression of hepatic E-selectin and higher levels of plasma sE-selectin. Further studies are required to investigate the role of E-selectin in the pathogenesis of NAFLD and the applicability of sE-selectin as a plasma biomarker of NAFLD/NASH. 


\section{Background}

Nonalcoholic fatty liver disease (NAFLD) is emerging as the principal cause of end-stage liver disease requiring transplantation. ${ }^{1}$ The pathogenesis is complex and involves genetic, inflammatory, (gut) microbiotic, metabolic, nutritional and lifestyle factors. ${ }^{2}$

Recent studies have implicated a role for liver sinusoidal endothelial cells in the pathogenesis of NAFLD. Liver sinusoidal endothelial dysfunction favors the development of steatosis and recruitment of inflammatory cells. ${ }^{3}$ This premise is largely based on animal studies, given the difficulties of acquiring human liver tissue to conduct endothelial function tests.

Plasma soluble E-selectin (sE-selectin) and vascular cell adhesion molecule-1 (sVCAM-1) have often been used as biomarkers of systemic endothelial activation. ${ }^{4-6}$ Of interest, we and others have previously shown that NAFLD is a significant determinant of plasma endothelial biomarkers, in particular $\mathrm{sE}$-selectin. ${ }^{7,8}$ Furthermore, it has been shown that hepatic E-selectin is actively involved in hepatic neutrophil infiltration in mice exposed to chronic plus binge ethanol feeding. ${ }^{9}$

The aim of the present study was, therefore, to study the relationship between NAFLD and sE-selectin in more detail. We aimed to assess: 1) the relationship between NAFLD and hepatic E-selectin expression; 2) the relationship between hepatic E-selectin expression and plasma sE-selectin levels; and 3) the association between NAFLD and plasma sE-selectin levels, independent of potential confounding factors. For this, a diverse set of studies was used, ranging from liver expression data in severely obese individuals and a mouse model of nonalcoholic steatohepatitis (NASH), to (genetic) epidemiology in two large cohorts.

\section{Methods}

Liver biopsies in severely obese individuals undergoing bariatric surgery

Details of this study have been described elsewhere. ${ }^{10,11}$ Briefly, liver, muscle and visceral adipose tissue (VAT) biopsies were collected from severely obese individuals (BMI $>40 \mathrm{~kg} / \mathrm{m}^{2}, \mathrm{n}=74$ ) undergoing elective bariatric surgery at the department of General Surgery of Maastricht University Medical Center 
(Maastricht, The Netherlands) between 2006 and 2009. Exclusion criteria were inflammatory or degenerative diseases, use of anti-inflammatory drugs, or alcohol intake $>10$ gram/day. All participants gave informed consent. The study was performed according to the Declaration of Helsinki ${ }^{12}$ and approved by the Medical Ethical Committee of Maastricht University Medical Center (Maastricht, The Netherlands). Liver biopsy samples were fixed in formalin and stained with Haematoxylin and Eosin (H\&E), periodic acid-Schiff with diastase and Masson's trichrome, and subsequently scored for the presence of steatosis, inflammation (i.e., lobular and portal inflammation and hepatocellular ballooning) and fibrosis by an experienced pathologist blinded for the study context and outcomes. Individuals were divided into three groups according to the NAFLD activity score (NAS): $\leq 2$ (no NASH), 3-4 (probable NASH) and $\geq 5$ (definite NASH). ${ }^{13}$ E-selectin and VCAM mRNA expression in liver, VAT and muscle were determined using Illumina HumanHT12 Bead-Chips (Illumina, San Diego, CA) and an Illumina BeadArray Reader. Plasma was drawn on the morning of the surgery after an overnight fast of 8 hours for measurement of various metabolic traits via routine clinical chemistry. Plasma levels of sEselectin and SVCAM were determined using a Diaclone ELISA kit (Diaclone SAS, Besançon Cedex, France). Histology, expression and plasma data were available in subsets of the study population; the number of individuals, therefore, differ between analyses.

\section{$\mathrm{LDLR}^{-/-}$mice fed a Western-type diet}

Female 16-18 weeks old C57BL/6 LDLR ${ }^{-/}$mice were fed a chow or Westerntype diet $(21 \%$ milk butter, $0,2 \%$ cholesterol, $46 \%$ carbohydrates [of which $40,5 \%$ sucrose] and $17 \%$ casein; SDSdiets \#824171) for 16 hours, 48 hours, 72 hours, 1 week or 3 weeks and were subsequently sacrificed via $\mathrm{CO}_{2} / \mathrm{O}_{2}$ inhalation. This Western-type diet is known to rapidly induce hepatic steatosis and inflammation in $\mathrm{LDLR}^{-/}$mice and has been used as model for NASH in numerous studies. ${ }^{14-16}$ Liver paraffin sections were stained for $\mathrm{H} \& \mathrm{E}$ and photographed using a Zeiss microscope (Axioskop 40) with a Jenoptik camera and Progress Capture Pro 2.8.8 software package. Hepatic inflammation (immune cell count and clustering, and hepatocyte injury) and steatosis (lipid droplets) were scored semi-quantitatively (0-4) in a blinded fashion by two independent experienced researchers. The average score was used for statistical analysis. RNA was isolated from the liver using Trizol reagent (Ambion). Next, E-selectin and TNF gene expression were determined using IQ SensiMix SYBR master mix (Bioline, London, UK) on a CFX96 Touch with CFX manager software (Biorad). The geometric mean of Cyclophillin and Beta2microglobulin was used as reference (see Supplementary Table S6.1 for primer 
sequences) and the $\triangle \triangle C T$ method was applied to calculate the gene expression levels. ${ }^{17}$ Plasma sE-selectin levels were determined using a DuoSet ELISA kit (R\&D Systems, Minneapolis, MN, USA). All experiments were approved by the Animal Experiments Committee of Maastricht University (Maastricht, The Netherlands) and in compliance with the relevant guidelines from the Directive 2010/63/EU of the European Parliament on the protection of animals used for scientific purposes.

\section{The combined Hoorn and CODAM studies}

The Hoorn and Cohort on Diabetes and Atherosclerosis Maastricht (CODAM) studies are observational, prospective cohort studies on determinants and (cardiovascular) complications of type 2 diabetes (T2D). The original Hoorn study was executed in 2,484 randomly selected residents of Hoorn between 1989 and 1992. ${ }^{18}$ Between 2000 and 2001, follow-up measurements were conducted in a subset $(n=822)$ of participants ${ }^{18}$, which were used for the current study. The CODAM study was performed in 574 participants of the Dutch Monitoring Project for Cardiovascular Diseases (MORGEN) and its predecessor with an elevated risk of T2D. ${ }^{19,20}$ All participants underwent a standard 75 gram oral glucose tolerance test to determine glucose metabolism state, i.e., normal glucose metabolism (NGM), impaired glucose metabolism (IGM) (comprising both impaired fasting glucose and impaired glucose tolerance) and T2D. All participants provided informed consent. The Hoorn and CODAM studies were performed according to the Declaration of Helsinki ${ }^{12}$ and approved by the local Medical Ethical Committees. As the protocols of both studies were similar, data was combined for statistical analyses, as has been done before..$^{21,22}$

In the combined cohorts, 1,286 individuals were available for genotyping. Genotyping of GCKR (rs1260326) and PNPLA3 (rs738409) was performed with validated Invitrogen TaqMan assays (Thermo Fisher Scientific, Waltham, MA). In CODAM, GCKR (rs1260326) was genotyped as part of a genome-wide association study array, using a HumanOmniExpress BeadChip (Illumina, San Diego, CA). ${ }^{21}$ Genotyping of rs1260326 and rs738409 was successful in 1,265 individuals. Both variants were in Hardy-Weinberg equilibrium $(p=0.93$ for rs1260326 [minor allele frequency: 38.5\%] and $p=0.16$ for rs738409 [minor allele frequency: $24.2 \%$ ] in individuals with NGM). Plasma was drawn after an overnight fast for measurement of amongst others alanine aminotransferase $(A L T)$, triglycerides, sE-selectin and SVCAM levels, as described before. ${ }^{18,20}$ Plasma levels of sE-selectin and SVCAM were determined using a MSD multiplex assay (Meso Scale Diagnostics, Rockville, Maryland), which is 
comparable to single-biomarker methods, such as ELISA, but could result in a different absolute concentration. ${ }^{23}$ In the Hoorn study, plasma ALT levels were only determined at the baseline visit (1989-1992), not during the follow-up measurements (2000-2001) that were used for the present study. The relationships of plasma ALT with sE-selectin and SVCAM levels were therefore studied in participants of the CODAM study only. Since genotype is timeindependent, the associations of rs1260326 and rs738409 with plasma ALT (and triglycerides, sE-selectin and SVCAM) were studied in the combined Hoorn and CODAM studies.

\section{Statistical analyses}

Data are presented as percentage of total, mean \pm SD or median (interquartile range). Non-normally distributed variables were log transformed before further analyses. Differences between groups or associations between variables were analyzed using One-way ANOVA or linear/logistic regression, respectively, with adjustments for age, sex and - if applicable - cohort (i.e., the Hoorn and CODAM studies). For readability purposes, figure data are presented after adjustment for age and sex (and cohort) in which the residuals are normalized to the average age and sex (and cohort) of that study population.

Multivariable linear regression was used to study the associations between plasma ALT and sE-selectin and SVCAM levels, independent of potential confounding factors, i.e., BMI, smoking, alcohol intake, plasma lipids (i.e., total cholesterol and HDL cholesterol), systolic blood pressure, HbA1c, use of glucose-lowering medication, use of lipid-modifying medication, inflammatory markers (i.e., C-reactive protein, amyloid A, interleukin-6, interleukin-8 and tumor necrosis factor $\alpha$ ) and history of cardiovascular disease. Finally, the associations between the NAFLD susceptibility genes PNPLA3 (rs738409) or GCKR (rs1260326) and plasma ALT, triglycerides, sE-selectin and sVCAM levels were analyzed under the assumption of an additive mode of inheritance, based on previous reports. ${ }^{24-26}$ Results were considered statistically significant at $p<0.05$. All statistical analyses were carried out by the IBM Statistical Package of Social Sciences (SPSS) version 23 for Windows (SPSS Inc., Chicago, IL). 


\section{Results}

\section{NAFLD histological stage is associated with hepatic E-selectin mRNA expression in severely obese individuals}

Hepatic E-selectin and VCAM mRNA expression patterns were studied in severely obese individuals undergoing bariatric surgery (see Table 6.1 for characteristics). Hepatic E-selectin mRNA expression increased with NAFLD severity from $7.05 \pm 0.22$ (NAS $\leq 2$ ), to $7.12 \pm 0.26$ (NAS 3-4) and to $7.24 \pm 0.23$ (NAS $\geq 5$ ) ( $p$ for trend $=0.02$ ), whereas an opposite trend was observed for hepatic VCAM mRNA expression (7.92 \pm 0.21 [NAS $\leq 2$ ], $7.77 \pm 0.28$ [NAS 3-4] and $7.80 \pm 0.21$ [NAS $\geq 5]$, $p$ for trend $=0.09)$. Hepatic E-selectin mRNA expression was associated with steatosis and fibrosis grade $(p=0.004$ and $\mathrm{p}=0.002$, respectively).

Table 6.1 General characteristics of severely obese individuals undergoing bariatric surgery and participants of the combined CODAM and Hoorn studies.

\begin{tabular}{lcc}
\hline & Bariatric surgery & CODAM/Hoorn \\
& $(\mathrm{n}=74)$ & $562 / 703$ \\
\hline Male/Female & $23 / 51$ & $64.6 \pm 8.4$ \\
Age $(\mathrm{y})$ & $44.5 \pm 9.8$ & $28.1 \pm 4.2$ \\
BMI $\left(\mathrm{kg} / \mathrm{m}^{2}\right)$ & $44.4(39.0-50.2)$ & $0.94 \pm 0.09$ \\
Waist-hip ratio & $0.97(0.89-1.11)$ & $33.2 \%$ \\
T2D (\%) & $34.9 \%$ & $5.9(5.3-6.8)$ \\
Glucose (mmol/L) & $5.8(5.3-7.2)$ & $5.9(5.6-6.3)$ \\
HbA1c (\%) & $6.2(5.6-6.8)$ & $66(46-100)$ \\
Insulin (pmol/L) & $111(69-160)$ & $10.3 \%$ \\
Glucose-lowering medication (\% yes) & $25.5 \%^{\ddagger}$ & $5.5 \pm 1.0$ \\
Total cholesterol (mmol/L) & $5.0 \pm 1.1$ & $3.4 \pm 0.9$ \\
LDL cholesterol (mmol/L) & $3.2 \pm 1.0$ & $1.2(1.0-1.5)$ \\
HDL cholesterol (mmol/L) & $0.9(0.7-1.1)$ & $1.4(1.0-1.9)$ \\
Triglycerides (mmol/L) & $1.75(1.23-2.63)$ & $17.5 \%$ \\
Lipid-modifying medication $(\%$ yes) & $25.5 \% \%^{\ddagger}$ & $22(17-28)^{\dagger}$ \\
ALT (U/L) & $22(17-31)$ & $20(16-24)^{\dagger}$ \\
AST (U/L) & $23(16-31)$ & $6.7(0.7-20.3)$ \\
Alcohol (g/day) & $\mathrm{nd}$ & $142 \pm 20$ \\
Systolic blood pressure (mmHg) & $138 \pm 18$ & $83 \pm 10$ \\
Diastolic blood pressure (mmHg) & $81 \pm 11$ & $38.9 \%$ \\
Antihypertensive medication $(\%$ yes) & $45.5 \% \%^{\ddagger}$ & $18.1 \%$ \\
Smoking (\% yes) & $30.8 \% \%^{\ddagger}$ & $42.4 \%$ \\
History of cardiovascular disease (\% yes) & $13.8 \% \%^{\ddagger}$ & \\
\hline
\end{tabular}

Data are expressed as percentage of total, mean $\pm \mathrm{SD}$ or median (interquartile range). ${ }^{\dagger}$ Presented for participants of the CODAM study only $(n=571)$. In the Hoorn study, plasma ALT levels have been measured at another visit, see Methods section; ${ }^{\ddagger} 20-50 \%$ of total study population $(n=74)$ variable unknown. Abbreviations: body mass index (BMI), normal glucose metabolism (NGM), impaired glucose metabolism (IGM), type 2 diabetes (T2D), hemoglobin A1c ( $\mathrm{HbA1c}$ ), low-density lipoprotein (LDL), high-density lipoprotein (HDL), alanine aminotransferase (ALT), aspartate aminotransferase (AST), not determined (nd). 
Hepatic E-selectin mRNA expression coincides with hepatic inflammation in LDLR $^{-/}$mice fed a Western-type diet

Since steatosis and inflammation often coincide, it is difficult to assess the independent relationship of each histological stage with hepatic E-selectin mRNA expression. We therefore determined hepatic E-selectin mRNA expression in $\mathrm{LDLR}^{-1-}$ mice fed a Western-type diet for 16 hours, 48 hours, 72 hours, 1 week and 3 weeks. We have previously shown that this model results in hepatic inflammation that precedes intrahepatic lipid accumulation ${ }^{15}$ and therefore allows a disentanglement of hepatic steatosis from inflammation. Indeed, the onset of hepatic steatosis was observed after 72 hours and increased until three weeks on a Western-type diet $(p=0.01$ and $p=0.001$ for 1 and 3 weeks versus chow diet, respectively, Figure 6.1, panel A), whereas hepatic inflammation reached its peak at one week, but appeared to decrease at week $3(p=0.001$ and $p=0.08$ for 1 and 3 weeks versus chow diet, respectively, Figure 6.1 , panel $B$ ). In line with this inflammation score, hepatic TNF mRNA expression was strongly upregulated only after one week Westerntype diet $(p<0.001$, Figure 6.1, panel C). Hepatic E-selectin mRNA expression followed a similar pattern and was only significantly different from the chow diet on week $1(p=0.001$, Figure 5.1 , panel $D)$. Of note, this was not the case for plasma sE-selectin levels, which already increased after 72 hours and remained stable thereafter (Supplementary Figure S6.1).

Hepatic E-selectin mRNA expression is associated with plasma sE-selectin levels in severely obese individuals

Analogous to the hepatic mRNA expression pattern, we observed a significantly positive relationship between NAS and plasma sE-selectin levels in severely obese individuals ( $p$ for trend $=0.003$, Figure 6.2 , panel $A$ ). In contrast to the negative trend between NAS and hepatic VCAM mRNA expression, a positive trend was now found for the relationship between NAS and plasma sVCAM levels $(p=0.07$, Figure 6.2 , panel $B)$. The discriminatory ability of $s E-$ selectin levels to distinguish NASH (i.e., NAS $\geq 5$ ) from non-NASH (i.e., NAS $\leq 2$ ) was statistically significant (area under receiver operating characteristic [ROC] curve: $0.73 ; 95 \% \mathrm{Cl}: 0.58 ; 0.88$, Supplementary Figure S6.2, panel A). Similar area under the curves were observed when the NAFLD histological stages were analyzed separately, except for fibrosis. Statistical significance was reached only for steatosis (Supplementary Figure S6.2, panel B-E). 
A
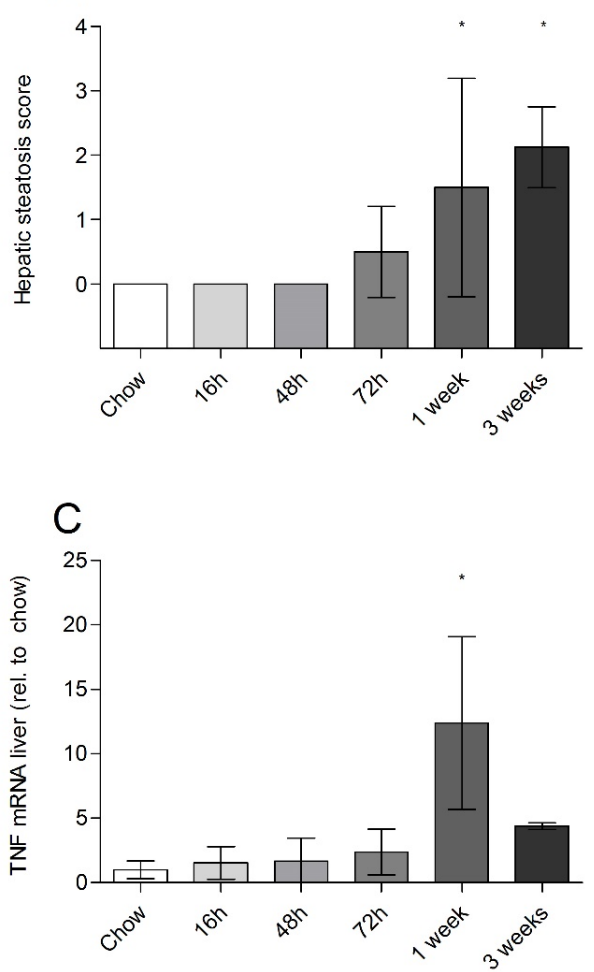

B

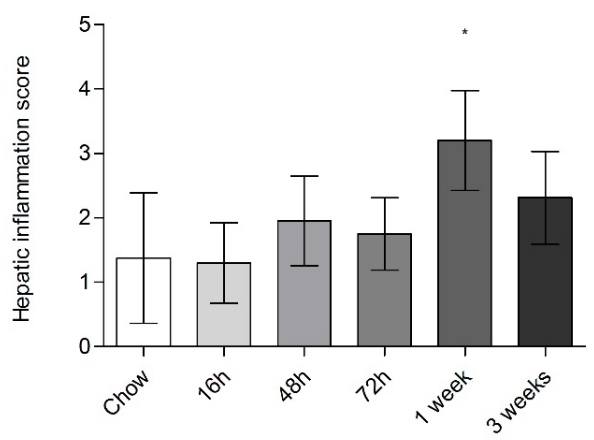

D

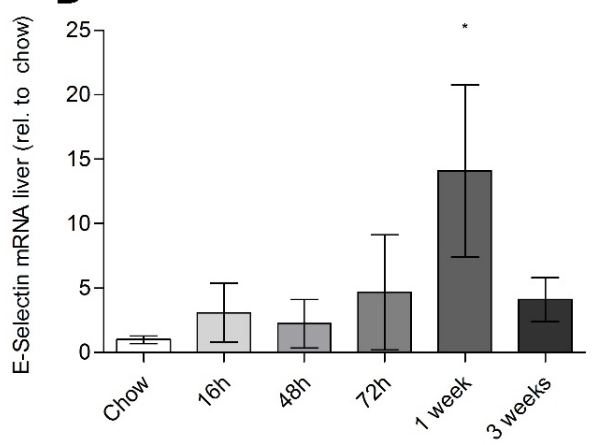

Figure 6.1 Liver steatosis score (A), inflammation score (B), hepatic TNF mRNA expression (C) and hepatic E-selectin mRNA expression (D) in LDLR ${ }^{-1-}$ mice fed a Western-type diet. Time points refer to time of sacrifice (see Methods section). $\mathrm{N}=5$ mice per group. Data are expressed as mean $\pm \mathrm{SD}$, analyzed with linear regression. ${ }^{*} p<0.05$ versus chow.

Hepatic E-selectin mRNA expression was significantly associated with plasma sE-selectin levels (unstandardized $\beta$ coefficient $=0.26$ [95\% Cl: 0.05;0.47], Figure 6.2, panel C). Such an association was not observed between hepatic VCAM mRNA expression and sVCAM levels $(\beta=0.02$ [95\% $\mathrm{Cl}:-0.08 ; 0.12]$, Figure 6.2, panel D). Visceral adipose tissue and muscle E-selectin mRNA expression patterns were not associated with plasma sE-selectin levels $(\beta=-$ 0.02 [95\% Cl: $-0.10 ; 0.05]$, Figure 6.2, panel $\mathrm{E}$; and $\beta=0.05$ [95\% Cl: $-0.22 ; 0.32]$, Figure 6.2, panel $\mathrm{F}$, respectively). 

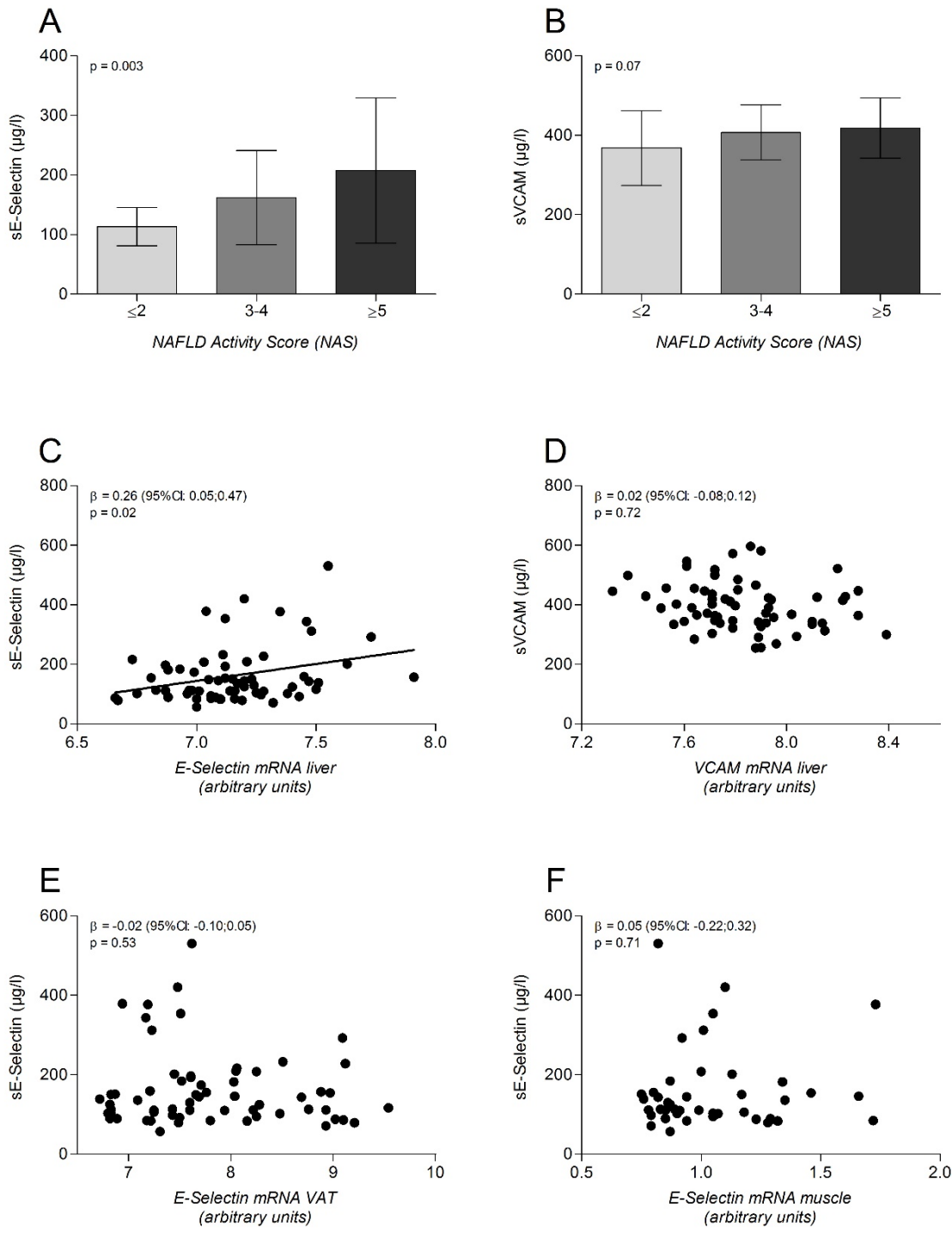

Figure 6.2 Relationship between E-selectin mRNA expression and plasma sE-selectin levels. Association between NAFLD severity (according to NAFLD activity score) and plasma sE-selectin levels (panel $A ; n=58$ ) and sVCAM levels (panel $B ; n=58$ ) in severely obese individuals; $E$-selectin mRNA expression in liver (panel $C ; n=62$ ), visceral adipose tissue (VAT) (panel $E ; n=62$ ) and muscle (panel $F ; n=43$ ) in relation to plasma sE-selectin levels. Hepatic VCAM mRNA expression in relation to plasma sVCAM levels (panel $D ; n=62$ ). Data are expressed as mean (adjusted for age and sex) $\pm S D$, analyzed with One-way ANOVA (panel A-B), or as individual data points (adjusted for age and sex), analyzed with linear regression (panel C-F). 


\section{Plasma ALT levels are independently associated with plasma sE-selectin levels in the CODAM study}

Linear regression models were constructed to determine the (independent) contribution of the liver to plasma sE-selectin levels at the population level, i.e., in the CODAM study $(n=571)$. Plasma ALT levels were associated with plasma $\mathrm{sE}$-selectin levels after adjustment for age and $\operatorname{sex}(\beta=0.35$ [95\% $\mathrm{Cl}: 0.27 ; 0.42]$, model 1, Table 6.2). The strength of association decreased after subsequent adjustment for BMI, smoking behaviour and alcohol intake, but remained statistically significant $(\beta=0.29$ [95\% Cl: $0.21 ; 0.37]$, model 2 , Table 6.2). BMI, alcohol intake and smoking behaviour were all independently associated with $\mathrm{sE}$-selectin levels (although the association for smoking behaviour was lost in the final model; Supplementary Table S6.2). Further adjustment for cardiovascular risk factors (model 3), inflammatory markers (model 4) and cardiovascular disease (model 5), did not essentially alter the relationship between plasma ALT and sE-selectin levels $(\beta=0.25$ [95\% $\mathrm{Cl}: 0.16 ; 0.34]$, model 5, Table 6.2). Plasma ALT levels were also independently associated with plasma sVCAM (Table 6.2, models 1-5). The strength of association almost halved after adjustment for inflammatory factors (Table 6.2, model 4), which was mainly accounted for by TNFa (data not shown).

Table 6.2 Multivariable regression analysis for the relationship of plasma ALT levels with plasma sE selectin or sVCAM levels in the CODAM study.

\begin{tabular}{llccccc}
\hline & \multicolumn{3}{c}{$\begin{array}{c}\text { Log plasma sE-selectin } \\
(\mathrm{n}=571)\end{array}$} & \multicolumn{2}{c}{$\begin{array}{c}\text { Log plasma sVCAM } \\
(\mathrm{n}=571)\end{array}$} \\
\cline { 2 - 7 } Model $^{\S}$ & $\beta$ & $95 \% \mathrm{Cl}$ & $\mathrm{p}$-Value & $\beta$ & $95 \% \mathrm{Cl}$ & $\mathrm{p}$-Value \\
\hline 1 & 0.346 & $0.271 ; 0.422$ & $<0.001$ & 0.112 & $0.071 ; 0.152$ & $<0.001$ \\
2 & 0.286 & $0.205 ; 0.367$ & $<0.001$ & 0.099 & $0.055 ; 0.143$ & $<0.001$ \\
3 & 0.264 & $0.177 ; 0.351$ & $<0.001$ & 0.101 & $0.055 ; 0.147$ & $<0.001$ \\
4 & 0.248 & $0.159 ; 0.338$ & $<0.001$ & 0.065 & $0.020 ; 0.110$ & 0.005 \\
5 & 0.249 & $0.160 ; 0.339$ & $<0.001$ & 0.066 & $0.021 ; 0.111$ & 0.004 \\
\hline
\end{tabular}

Analyzed with linear regression. Beta's represent unstandardized regression coefficients. ${ }^{\S}$ Model 1: adjusted for age + sex. Model 2: adjusted for model $1+$ BMI, smoking and alcohol intake. Model 3: adjusted for model $2+$ cardiovascular risk factors (total cholesterol, HDL cholesterol, systolic blood pressure, HbA1c, use of glucoselowering medication and use of lipid-modifying medication). Model 4: adjusted for model $3+$ inflammatory markers (C-reactive protein, amyloid A, interleukin-6, interleukin-8 and tumor necrosis factor $\alpha$ ). Model 5: adjusted for model $4+$ history of cardiovascular disease. 
NAFLD susceptibility genes co-segregate with plasma sE-selectin levels in the CODAM and Hoorn studies

To further examine the relationship between NAFLD and plasma sE-selectin levels, independent of potential confounders, we studied the association of two NAFLD susceptibility genes, i.e., PNPLA3 (rs738409) and GCKR (rs1260326), with plasma sE-selectin levels in the combined CODAM and Hoorn studies $(n=1,265$, see Table 6.1 for characteristics). Previous studies have shown that both genetic variants predispose to NAFLD (including NASH) and elevated liver enzymes ${ }^{26-28}$, but have opposing effects on factors that are associated with systemic endothelial activation, i.e., plasma lipids ${ }^{24,29}, \mathrm{~T}^{2} \mathrm{D}^{30}$ and coronary artery disease risk ${ }^{31,32}$ (Supplementary Table S6.3). Indeed, positive trends were observed for the relationships of the rs1260326 T-allele (GCKR) and rs738409 G-allele (PNPLA3) with plasma ALT levels $(\beta=0.011$ [95\% Cl: $-0.004 ; 0.26$ ], $p=0.15$; and $\beta=0.023$ [95\%Cl: $0.005 ; 0.041$ ], $p=0.01$, respectively, Table 6.3), whereas opposing effects were found for plasma triglycerides levels $(\beta=0.017$ [95\%Cl: $0.006 ; 0.027$ ], $p=0.002$; and $\beta=-0.016$ [95\% Cl: $-0.029 ;-0.004$ ], $p=0.009$, respectively, Table 6.3). Statistically significant, positive associations were observed for both GCKR and PNPLA3 with plasma sE-selectin levels ( $\beta=0.019$ [95\%Cl: $0.006 ; 0.032$ ], $p=0.004 ;$ and $\beta=0.020 ;[95 \% \mathrm{Cl}: 0.005 ; 0.036$ ], $p=0.01$, respectively, Table 6.3$)$, but not with plasma sVCAM levels $(\beta=0.001$ [95\% Cl: $-0.005 ; 0.008$ ], $p=0.69$; and $\beta=0.006$ [95\% $\mathrm{Cl}:-0.002 ; 0.014], p=0.13$, respectively, Table 6.3). Additional adjustment for glucose metabolism state (i.e., NGM, IGM and T2D) did not affect the outcomes (data not shown).

Table 6.3 Relationship between NAFLD susceptibility genes and plasma ALT, triglycerides, sE selectin and sVCAM levels in the combined CODAM and Hoorn studies.

\begin{tabular}{lcccc}
\hline & \multicolumn{2}{c}{ Effect size of NAFLD risk allele } \\
& $\begin{array}{c}\text { PNPLA3 (rs738409 G-allele) } \\
(\mathrm{n}=1,265)\end{array}$ & $\begin{array}{c}\text { (rs1260326 T-allele) } \\
(\mathrm{n}=1,265)\end{array}$ \\
\cline { 2 - 5 } & $\beta$ & $95 \% \mathrm{Cl}$ & $95 \% \mathrm{Cl}$ \\
\hline Log ALT & 0.023 & $0.005 ; 0.041$ & 0.011 & $-0.004 ; 0.026$ \\
Log Triglycerides & -0.016 & $-0.029 ;-0.004$ & 0.017 & $0.006 ; 0.027$ \\
Log sE-selectin & 0.020 & $0.005 ; 0.036$ & 0.019 & $0.006 ; 0.032$ \\
Log sVCAM & 0.006 & $-0.002 ; 0.014$ & 0.001 & $-0.005 ; 0.008$ \\
\hline
\end{tabular}

Analyzed with linear regression, under the assumption of an additive mode of inheritance. Adjusted for age, sex and cohort. Beta's represent unstandardized regression coefficients. 


\section{Discussion}

Soluble E-selectin has frequently been used as a plasma biomarker of endothelial dysfunction in epidemiological studies. In the present study, we showed that: 1) NAFLD severity, in particular the inflammatory stage, was associated with hepatic E-selectin expression; 2) E-selectin mRNA expression in liver, but not in VAT and muscle, was associated with plasma sE-selectin levels; and 3) NAFLD susceptibility genes and liver parenchyma damage (reflected by plasma ALT levels) were associated with plasma sE-selectin levels, independent of potential confounding factors. Such consistent associations were not observed for sVCAM.

Animal studies have shown that liver sinusoidal endothelial cells are involved in the pathogenesis of NAFLD. ${ }^{3}$ In general, the microvascular endothelium is a major regulator of vasomotor tone, permeability, coagulation, fibrinolysis and smooth muscle cell proliferation. Endothelial dysfunction occurs when one of these functions is impaired. E-selectin is an adhesion molecule that is specifically expressed on cytokine-activated endothelial cells. It mediates the adhesion and rolling of leukocytes on the endothelium as a part of the inflammatory response. Shedding of E-selectin from the damaged and/or cytokine activated endothelial cells results in its release (as soluble E-selectin) into the circulation, which can subsequently be measured as a biomarker of endothelial activation. ${ }^{33}$

Previous experiments have shown that E-selectin is actively involved in the development of hepatic inflammation in mice exposed to chronic plus binge ethanol feeding. ${ }^{9}$ In the present study, we noted an increased hepatic Eselectin mRNA expression in parallel to the inflammatory response in $\mathrm{LDLR}^{-1-}$ mice fed a Western-type diet, an established mouse model of rapidly-induced $\mathrm{NASH} .{ }^{15,16}$ Such a pattern was not observed in plasma, which may be due to the coexistence of systemic inflammation in this mouse model. ${ }^{34} \mathrm{~A}$ similar relationship between NAFLD severity and hepatic E-selectin mRNA expression was observed in severely obese individuals undergoing bariatric surgery. Although we did not address which cell type was responsible for the greater hepatic E-selectin mRNA expression in our studies, e.g., hepatocytes, stellate cells, Kupffer cells or endothelial cells, previous immunohistochemical studies have shown that E-selectin protein was specifically expressed on the vascular endothelium in different inflammatory liver diseases (i.e., alcoholic fatty liver disease, allograft rejection and primary biliary cirrhosis), but not in normal livers. ${ }^{35}$ 
In the present study, hepatic E-selectin mRNA expression, but not VAT or muscle E-selectin mRNA expression, was associated with plasma sE-selectin levels. We did not find an association between hepatic VCAM mRNA expression and plasma sVCAM levels. These findings raise the question on whether (the activated endothelium in) NAFLD could be a source of circulating sE-selectin. Although plasma sE-selectin was able to discriminate NASH from non-NASH, the area under the ROC curve of 0.73 is too small to use $\mathrm{sE}$-selectin as a single, diagnostic biomarker of NASH. Future studies are needed to ascertain whether inclusion of sE-selectin in a multiple biomarker risk score, e.g., in combination with cytokeratin-18 fragment levels ${ }^{36}$, can be of clinical value.

In the present study we corroborated the previously reported, strong relationship between NAFLD and sE-selectin. ${ }^{7,8}$ In addition, by using large cohorts we are able to adjust for potential confounding factors, such as smoking, low-grade inflammation, plasma lipids, type 2 diabetes and cardiovascular disease, which have all been associated with systemic endothelial dysfunction. ${ }^{4,5,37-39}$ We observed that plasma ALT levels were associated with sE-selectin, independent of these potential confounders. The greatest decrease in the regression coefficient was observed when alcohol, $\mathrm{BMI}$ and smoking behavior were added to the model, which is in contrast to the regression models for sVCAM where inflammatory markers, in particular TNFa, had the greatest effect on the regression coefficient. The independent association of alcohol on sE-selectin levels might be explained by a direct effect of alcohol on the hepatic endothelium, as previously shown. ${ }^{9}$

The association of NAFLD with sE-selectin, independent of factors that are associated with systemic endothelial activation, was also demonstrated by the use of common NAFLD susceptibility genes, i.e., PNPLA3 and GCKR. ${ }^{27}$ Since individuals are 'randomized' at conception to receive an allele that either predisposes to or protects from NAFLD, these genetic variants can serve as instruments to make causal inferences about the relationship between NAFLD and SE-selectin levels. Although pleiotropic effects have been reported for both PNPLA3 and GCKR - which may violate this 'Mendelian randomization' assumption ${ }^{40}$ - it should be noted that these variants have opposing pleiotropic effects on plasma lipids ${ }^{24,29}$ and risk of type 2 diabetes and coronary artery disease $^{31,32,41}$, as summarized in Supplementary Table S6.3. As a combination, they therefore serve as a model to disentangle NAFLD from factors that affect systemic endothelial function, despite the presence of pleiotropy. Again, we observed that sE-selectin levels co-segregated with plasma ALT levels, not with plasma triglycerides. These associations were not found for SVCAM. 
This study has several strengths and limitations. A major strength is the adoption of different methodologies, i.e., animal experiments, liver biopsy studies and cohort studies (with both classical biomarkers and the use of distinctive genetic markers), that enabled us to demonstrate that the liver, more specifically NAFLD, is closely linked to circulating sE-selectin levels. The observational nature of our studies does, however, not allow to exactly determine if and to what extent $s E$-selectin is derived from the liver. Other organs besides the inflamed liver can contribute to circulating $s E-s e l e c t i n$ as well. In the present study, plasma ALT was used as a liver-specific biomarker of parenchymal damage. Previous studies have shown that plasma ALT is not a perfect biomarker of NAFLD severity, although simple steatosis and NASH have been associated with greater plasma ALT levels. ${ }^{42,43}$ It is therefore likely that there is a residual 'NAFLD-effect' on sE-selectin levels when plasma ALT is entered as an independent variable in the regression model. This phenomenon could explain the significant, independent association of BMI - a strong determinant of NAFLD ${ }^{44}$ - with sE-selectin levels in our study.

In conclusion, the present study shows that higher expression of hepatic E-selectin and higher levels of plasma sE-selectin are associated with NAFLD and related markers. These findings favor further study to elucidate the role of E-selectin in the pathogenesis of NAFLD and the applicability of sE-selectin as a plasma biomarker of NAFLD/NASH. 


\section{Literature}

1. Charlton MR, Burns JM, Pedersen RA, Watt KD, Heimbach JK, Dierkhising RA. Frequency and outcomes of liver transplantation for nonalcoholic steatohepatitis in the United States. Gastroenterology 2011;141:1249-53.

2. Haas JT, Francque S, Staels B. Pathophysiology and Mechanisms of Nonalcoholic Fatty Liver Disease. Annu Rev Physiol 2016;78:181-205.

3. Hammoutene A, Rautou PE. Role of liver sinusoidal endothelial cells in non-alcoholic fatty liver disease. J Hepatol 2019;70:1278-91.

4. Meigs JB, Hu FB, Rifai N, Manson JE. Biomarkers of endothelial dysfunction and risk of type 2 diabetes mellitus. JAMA 2004;291:1978-86.

5. Hwang SJ, Ballantyne CM, Sharrett AR, et al. Circulating adhesion molecules VCAM-1, ICAM-1, and E-selectin in carotid atherosclerosis and incident coronary heart disease cases: the Atherosclerosis Risk In Communities (ARIC) study. Circulation 1997;96:4219-25.

6. Herder C, Baumert J, Zierer A, et al. Immunological and cardiometabolic risk factors in the prediction of type 2 diabetes and coronary events: MONICA/KORA Augsburg case-cohort study. PLoS One 2011;6:e19852.

7. Brouwers MC, Govers-Riemslag J, Schalkwijk CG, et al. Plasma PAI-1 levels are independently related to fatty liver and hypertriglyceridemia in familial combined hyperlipidemia, involvement of apolipoprotein E. Thromb Res 2008;122:466-72.

8. Musso G, Gambino R, Bo S, et al. Should nonalcoholic fatty liver disease be included in the definition of metabolic syndrome? A cross-sectional comparison with Adult Treatment Panel III criteria in nonobese nondiabetic subjects. Diabetes Care 2008;31:562-8.

9. Bertola A, Park O, Gao B. Chronic plus binge ethanol feeding synergistically induces neutrophil infiltration and liver injury in mice: a critical role for E-selectin. Hepatology 2013; 58:1814-23.

10. Rensen SS, Slaats $\mathrm{Y}$, Nijhuis J, et al. Increased hepatic myeloperoxidase activity in obese subjects with nonalcoholic steatohepatitis. Am J Pathol 2009;175:1473-82.

11. Wolfs MG, Rensen SS, Bruin-Van Dijk EJ, et al. Co-expressed immune and metabolic genes in visceral and subcutaneous adipose tissue from severely obese individuals are associated with plasma HDL and glucose levels: a microarray study. BMC Med Genomics 2010;3:34.

12. WHO. World Medical Association Declaration of Helsinki: ethical principles for medical research involving human subjects. JAMA 2013;310:2191-4.

13. Kleiner DE, Brunt EM, Van Natta M, et al. Design and validation of a histological scoring system for nonalcoholic fatty liver disease. Hepatology 2005;41:1313-21.

14. Bijnen M, Josefs $T$, Cuijpers I, et al. Adipose tissue macrophages induce hepatic neutrophil recruitment and macrophage accumulation in mice. Gut 2018;67:1317-27.

15. Wouters K, van Gorp PJ, Bieghs V, et al. Dietary cholesterol, rather than liver steatosis, leads to hepatic inflammation in hyperlipidemic mouse models of nonalcoholic steatohepatitis. Hepatology 2008;48:474-86.

16. Subramanian S, Goodspeed L, Wang S, et al. Dietary cholesterol exacerbates hepatic steatosis and inflammation in obese LDL receptor-deficient mice. J Lipid Res 2011;52: 1626-35.

17. Livak KJ, Schmittgen TD. Analysis of relative gene expression data using real-time quantitative PCR and the 2(-Delta Delta C(T)) Method. Methods 2001;25:402-8.

18. Rutters F, Nijpels G, Elders P, et al. Cohort Profile: The Hoorn Studies. Int J Epidemiol 2018;47:396-j.

19. Kershaw KN, Droomers M, Robinson WR, Carnethon MR, Daviglus ML, Monique Verschuren WM. Quantifying the contributions of behavioral and biological risk factors to socioeconomic disparities in coronary heart disease incidence: the MORGEN study. Eur J Epidemiol 2013;28:807-14. 
20. Kruijshoop M, Feskens EJ, Blaak EE, de Bruin TW. Validation of capillary glucose measurements to detect glucose intolerance or type 2 diabetes mellitus in the general population. Clin Chim Acta 2004;341:33-40.

21. Simons N, Dekker JM, van Greevenbroek MM, et al. A Common Gene Variant in Glucokinase Regulatory Protein Interacts With Glucose Metabolism on Diabetic Dyslipidemia: the Combined CODAM and Hoorn Studies. Diabetes Care 2016;39:1811-7.

22. Geelen CC, van Greevenbroek MM, van Rossum EF, et al. Bcll glucocorticoid receptor polymorphism is associated with greater body fatness: the Hoorn and CODAM studies. J Clin Endocrinol Metab 2013;98:E595-9.

23. van Bussel BC, Ferreira I, van de Waarenburg MP, et al. Multiple inflammatory biomarker detection in a prospective cohort study: a cross-validation between well-established singlebiomarker techniques and an electrochemiluminescense-based multi-array platform. PLoS One 2013;8:e58576.

24. Vaxillaire M, Cavalcanti-Proenca C, Dechaume A, et al. The common P446L polymorphism in GCKR inversely modulates fasting glucose and triglyceride levels and reduces type 2 diabetes risk in the DESIR prospective general French population. Diabetes 2008;57:2253-7.

25. Romeo S, Kozlitina J, Xing C, et al. Genetic variation in PNPLA3 confers susceptibility to nonalcoholic fatty liver disease. Nat Genet 2008;40:1461-5.

26. Mancina RM, Dongiovanni P, Petta S, et al. The MBOAT7-TMC4 Variant rs641738 Increases Risk of Nonalcoholic Fatty Liver Disease in Individuals of European Descent. Gastroenterology 2016;150:1219-30.e6.

27. Speliotes EK, Yerges-Armstrong LM, Wu J, et al. Genome-wide association analysis identifies variants associated with nonalcoholic fatty liver disease that have distinct effects on metabolic traits. PLoS Genetics 2011;7:e1001324.

28. Lin YC, Chang PF, Chang MH, Ni YH. Genetic variants in GCKR and PNPLA3 confer susceptibility to nonalcoholic fatty liver disease in obese individuals. Am J Clin Nutr 2014;99:869-74.

29. Liu DJ, Peloso GM, Yu H, et al. Exome-wide association study of plasma lipids in $>300,000$ individuals. Nat Genet 2017;49:1758-66.

30. Mahajan A, Wessel J, Willems SM, et al. Refining the accuracy of validated target identification through coding variant fine-mapping in type 2 diabetes. Nat Genet 2018;50: $559-71$.

31. Simons N, Isaacs A, Koek GH, Kuc S, Schaper NC, Brouwers M. PNPLA3, TM6SF2, and MBOAT7 Genotypes and Coronary Artery Disease. Gastroenterology 2017;152:912-3.

32. Simons P, Simons N, Stehouwer CDA, Schalkwijk CG, Schaper NC, Brouwers M. Association of common gene variants in glucokinase regulatory protein with cardiorenal disease: A systematic review and meta-analysis. PloS One 2018;13:e0206174.

33. Roldan V, Marin F, Lip GY, Blann AD. Soluble E-selectin in cardiovascular disease and its risk factors. A review of the literature. Thromb Haemost 2003;90:1007-20.

34. Ishibashi S, Goldstein JL, Brown MS, Herz J, Burns DK. Massive xanthomatosis and atherosclerosis in cholesterol-fed low density lipoprotein receptor-negative mice. J Clin Invest 1994;93:1885-93.

35. Adams DH, Hubscher SG, Fisher NC, Williams A, Robinson M. Expression of E-selectin and E-selectin ligands in human liver inflammation. Hepatology 1996;24:533-8.

36. Wieckowska A, Zein NN, Yerian LM, Lopez AR, McCullough AJ, Feldstein AE. In vivo assessment of liver cell apoptosis as a novel biomarker of disease severity in nonalcoholic fatty liver disease. Hepatology 2006;44:27-33.

37. Cui M, Cui R, Liu K, et al. Associations of Tobacco Smoking with Impaired Endothelial Function: The Circulatory Risk in Communities Study (CIRCS). J Atheroscler Thromb 2018;25:836-45.

38. Fichtlscherer S, Rosenberger G, Walter DH, Breuer S, Dimmeler S, Zeiher AM. Elevated Creactive protein levels and impaired endothelial vasoreactivity in patients with coronary artery disease. Circulation 2000;102:1000-6. 
39. Vita JA, Treasure CB, Nabel EG, et al. Coronary vasomotor response to acetylcholine relates to risk factors for coronary artery disease. Circulation 1990;81:491-7.

40. Burgess S, Foley CN, Zuber V. Inferring Causal Relationships Between Risk Factors and Outcomes from Genome-Wide Association Study Data. Annu Rev Genomics Hum Genet 2018;19:303-27.

41. Mahajan A, Wessel J, Willems SM, et al. Refining the accuracy of validated target identification through coding variant fine-mapping in type 2 diabetes. Nat Genet 2018;50: $559-71$.

42. Neuschwander-Tetri BA, Clark JM, Bass NM, et al. Clinical, laboratory and histological associations in adults with nonalcoholic fatty liver disease. Hepatology 2010;52:913-24.

43. Maximos M, Bril F, Portillo Sanchez $P$, et al. The role of liver fat and insulin resistance as determinants of plasma aminotransferase elevation in nonalcoholic fatty liver disease. Hepatology 2015;61:153-60.

44. Wanless IR, Lentz JS. Fatty liver hepatitis (steatohepatitis) and obesity: an autopsy study with analysis of risk factors. Hepatology 1990;12:1106-10. 


\section{Supplementary Materials}

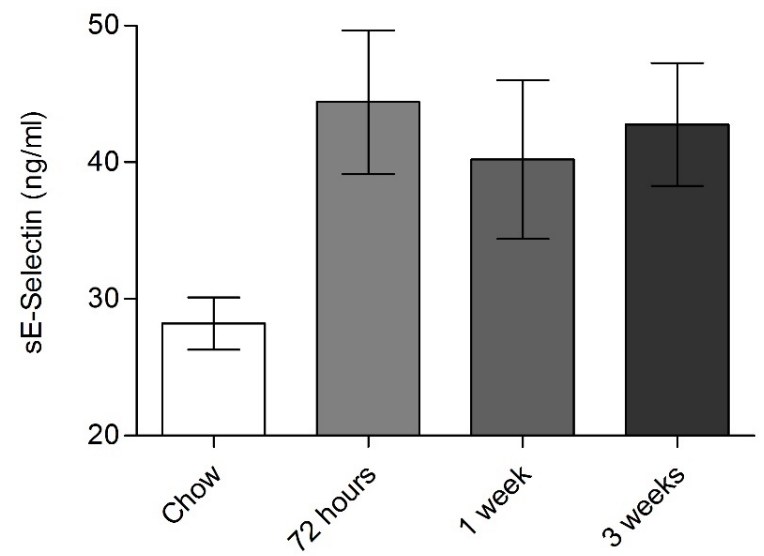

Figure S6.1 Plasma sE-selectin levels in LDLR ${ }^{-1-}$ mice on a Western-type diet. Plasma sE-selectin level in $\mathrm{LDLR}^{-/-}$mice fed a Chow diet $(n=5)$ or Western-type diet for 72 hours $(n=5)$, 1 week $(n=5)$ and 3 weeks $(n=4)$. Data are expressed as mean $\pm S D$, analyzed with linear regression. ${ }^{*} p<0.05$ versus chow. 

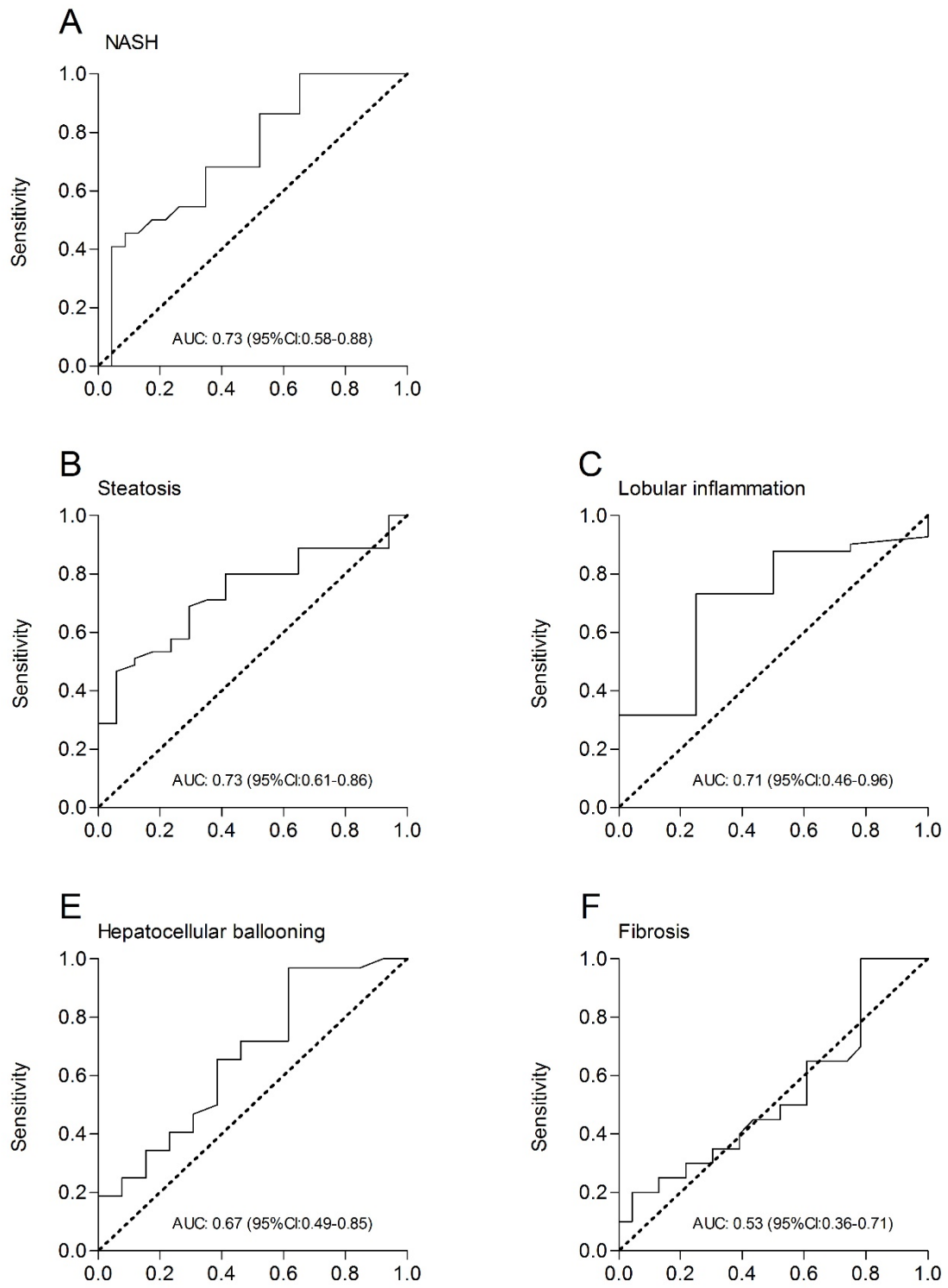

Figure S6.2 Receiver operating characteristic curves for sE-selectin levels to discriminate between NASH and the individual NAFLD histological stages in severely obese individuals. Receiver operating characteristic curves for $s E$-selectin levels to discriminate between NASH (NAS $\geq 5$ ) and no NASH (NAS $\leq 2)(n=45$, panel A) and the individual NAFLD histological stages (i.e., steatosis $(n=62$, panel B), lobular inflammation $(n=45$, panel C), ballooning $(n=45$, panel $D)$ and fibrosis $(n=43$, panel E)) in severely obese individuals. Abbreviations: area under the curve (AUC), nonalcoholic fatty liver disease activity score (NAS), nonalcoholic steatohepatitis (NASH), nonalcoholic fatty liver disease (NAFLD). 
Table S6.1 Primer sequences.

\begin{tabular}{lll}
\hline Gene & FW primer & RV primer \\
\hline TNF & CATCTTCTCAAAATTCGAGTGACAA & TGGGAGTAGACAAGGTACAACCC \\
E-selectin & AGCAGAGTTTCACGTTGCAGG & TGGCGCAGATAAGGCTTCA \\
Cyclophilin & TTCCTCCTTTCACAGAATTATTCCA & CCGCCAGTGCCATTATGG \\
B2-Micro & CTTTCTGGTGCTTGTCTCACTGA & GTATGTTCGGCTTCCCATTCTC \\
\hline
\end{tabular}

Forward and reverse qPCR primer sequences of all measured genes.

Table S6.2 Determinants of plasma sE-selectin levels in participants of the CODAM study.

\begin{tabular}{lcc}
\hline & \multicolumn{2}{c}{$\begin{array}{c}\text { CODAM Study } \\
\text { Total population ( } \mathrm{n}=571) \\
\text { log plasma sE-selectin }\end{array}$} \\
\hline Age & \multicolumn{1}{c}{$95 \% \mathrm{Cl}$} \\
\cline { 2 - 3 } Sex & 0.001 & $-0.001 ; 0.003$ \\
BMI & 0.025 & $-0.006 ; 0.056$ \\
Smoking & 0.005 & $0.001 ; 0.008^{*}$ \\
Alcohol & 0.021 & $-0.012 ; 0.053$ \\
ALT & 0.028 & $0.004 ; 0.052^{*}$ \\
Total cholesterol & 0.249 & $0.160 ; 0.339^{*}$ \\
HDL cholesterol & 0.002 & $-0.011 ; 0.015$ \\
Systolic blood pressure & -0.225 & $-0.450 ; 0.000$ \\
HbA1c & 0.000 & $-0.000 ; 0.001$ \\
Use of glucose-lowering medication & 0.005 & $-0.337 ; 0.346$ \\
Use of lipid-modifying medication & -0.010 & $-0.055 ; 0.035$ \\
C-reactive protein & 0.002 & $-0.033 ; 0.037$ \\
Amyloid A & 0.008 & $-0.051 ; 0.067$ \\
Interleukin-6 & 0.004 & $-0.076 ; 0.084$ \\
Interleukin-8 & 0.018 & $-0.077 ; 0.113$ \\
Tumor necrosis factor $\alpha$ & 0.099 & $0.009 ; 0.189^{*}$ \\
History of cardiovascular disease & -0.023 & $-0.137 ; 0.091$ \\
\hline
\end{tabular}

Analyzed with linear regression. Beta's represent unstandardized regression coefficients. ${ }^{*} p<0.05$.

Table S6.3 Overview of previously reported associations of PNPLA3 and GCKR with NAFLD and factors that have been associated with systemic endothelial activation.

\begin{tabular}{lccc}
\hline & PNPLA3 & GCKR & \\
& $\begin{array}{c}\text { rs738409 } \\
\text { G-allele }\end{array}$ & $\begin{array}{c}\text { rs1260326 } \\
\text { T-allele }\end{array}$ & Reference \\
\hline Nonalcoholic fatty liver disease (including NASH) & $\uparrow$ & $\uparrow$ & 1,2 \\
Plasma lipids & $\downarrow$ & $\uparrow$ & 3,4 \\
Type 2 diabetes & $\uparrow$ & $\downarrow$ & 5 \\
Coronary artery disease & $\downarrow$ & $\uparrow$ & 6,7 \\
\hline
\end{tabular}




\section{Literature}

1. Romeo S, Kozlitina J, Xing C, et al. Genetic variation in PNPLA3 confers susceptibility to nonalcoholic fatty liver disease. Nat Genet 2008;40:1461-5.

2. Lin YC, Chang PF, Chang MH, Ni YH. Genetic variants in GCKR and PNPLA3 confer susceptibility to nonalcoholic fatty liver disease in obese individuals. Am J Clin Nutr 2014;99: 869-74.

3. Vaxillaire M, Cavalcanti-Proenca C, Dechaume A, et al. The common P446L polymorphism in GCKR inversely modulates fasting glucose and triglyceride levels and reduces type 2 diabetes risk in the DESIR prospective general French population. Diabetes 2008;57:2253-7.

4. Liu DJ, Peloso GM, Yu H, et al. Exome-wide association study of plasma lipids in $>300,000$ individuals. Nat Genet 2017;49:1758-66.

5. Mahajan A, Wessel J, Willems SM, et al. Refining the accuracy of validated target identification through coding variant fine-mapping in type 2 diabetes. Nat Genet 2018;50: $559-71$.

6. Simons N, Isaacs A, Koek GH, Kuc S, Schaper NC, Brouwers M. PNPLA3, TM6SF2, and MBOAT7 Genotypes and Coronary Artery Disease. Gastroenterology 2017;152:912-3.

7. Simons P, Simons N, Stehouwer CDA, Schalkwijk CG, Schaper NC, Brouwers M. Association of common gene variants in glucokinase regulatory protein with cardiorenal disease: A systematic review and meta-analysis. PloS One 2018;13:e0206174. 
Modulation of fructose metabolism to study the relationship between intrahepatic lipid content and cardiovascular disease 



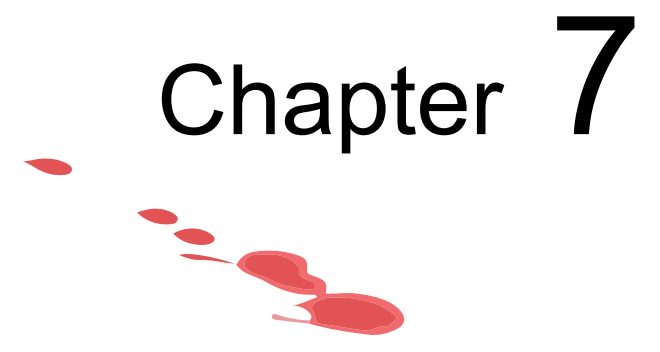

Intrahepatic lipid content in hereditary fructose intolerance
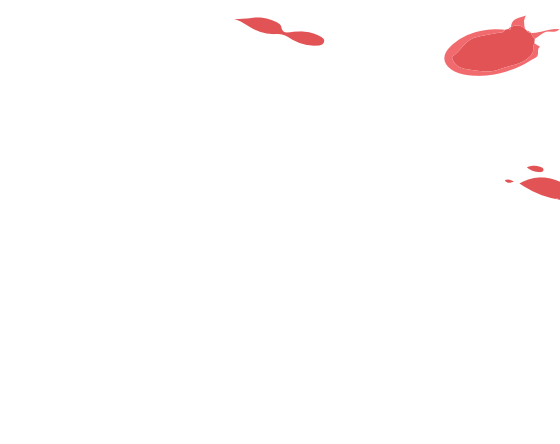

Patients with aldolase B deficiency are characterized by an increased intrahepatic lipid content

Nynke Simons, François-Guillaume Debray, Nicolaas C. Schaper, M. Eline Kooi, Edith J.M. Feskens, Carla E.M. Hollak, Lucas Lindeboom, Ger H. Koek, Judith A.P. Bons, Dirk J Lefeber, Leanne Hodson, Casper G. Schalkwijk, Coen D.A. Stehouwer, David Cassiman, J Clin Endocrinol Metab. 2019;104(11):5056-5064 


\section{Abstract}

\section{Background}

There is an ongoing debate on whether and how fructose is involved in the pathogenesis of nonalcoholic fatty liver disease (NAFLD). A recent experimental study showed an increased intrahepatic lipid (IHL) content in mice deficient for aldolase $\mathrm{B}$ (aldo $\mathrm{B}^{-/-}$), the enzyme that converts fructose-1phosphate to triose phosphates. The aim of the present study was to translate these experimental findings to the human situation.

\section{Methods}

Fifteen patients with hereditary fructose intolerance, a rare inborn error of metabolism caused by a defect in aldolase $B$, were recruited from the national metabolic clinics and compared to healthy subjects matched for age, sex and body mass index (BMI). IHL content was assessed by proton magnetic resonance spectroscopy.

\section{Results}

IHL content was higher in aldo $\mathrm{B}^{-/-}$patients compared to controls $(2.5 \%$ versus $0.6 \%, p=0.001$ ), at a background of a lean body mass (median BMI: 20.4 and $21.8 \mathrm{~kg} / \mathrm{m}^{2}$, respectively). Glucose excursions during an oral glucose load were higher in aldo $B^{-/-}$patients $(p=0.043)$. Hypoglycosylated transferrin, a surrogate marker for hepatic fructose-1-phosphate concentrations, was more abundant in aldo $B^{-/-}$patients than in controls $(p<0.001)$. Finally, plasma betahydroxybutyrate, a biomarker of hepatic beta-oxidation, was lower in aldo $\mathrm{B}^{-/-}$ patients compared to controls $(p=0.009)$.

\section{Conclusions}

This study extends previous experimental findings by demonstrating that aldolase $B$ deficiency also results in IHL accumulation in humans. It suggests that the accumulation of fructose-1-phosphate and impairment of betaoxidation are involved in the pathogenesis. 


\section{Background}

In parallel with the current obesity epidemic, nonalcoholic fatty liver disease (NAFLD) has emerged as a threat to global health. ${ }^{1}$ While NAFLD can lead to end-stage liver failure and hepatocellular carcinoma, individuals are also at risk for extrahepatic complications, such as dyslipidemia, type 2 diabetes, chronic kidney disease and cardiovascular disease. ${ }^{2}$

The pathophysiology of NAFLD is complex and involves genetic, inflammatory, (gut) microbiotic, metabolic, nutritional and lifestyle factors. ${ }^{3}$ A recent dietary intervention study showed that overfeeding with saturated fat or carbohydrates increased intrahepatic lipid (IHL) content, albeit by different mechanisms: the former by stimulating fatty acid flux from adipose tissue towards the liver, the latter by increasing hepatic de novo lipogenesis. ${ }^{4}$

There is an ongoing discussion on whether the type of carbohydrate, i.e., glucose or fructose, matters. Although fructose is preferentially metabolized in the liver ${ }^{5}$, several epidemiological studies have failed to demonstrate that increased fructose intake is associated with increased $\mathrm{IHL}$ content. ${ }^{6,7}$ Furthermore, intervention studies comparing added glucose versus added fructose, under either isocaloric or hypercaloric conditions, did not observe any difference in $\mathrm{IHL}$ content either. ${ }^{8,9}$ On the other hand, other experimental studies have shown that added fructose, not glucose, specifically increases de novo lipogenesis and plasma triglycerides. ${ }^{10,11}$

The catabolism of fructose in the liver involves a two-step process: 1) phosphorylation of fructose by ketohexokinase and 2) aldolase $B$ mediated conversion of fructose-1-phoshpate $(\mathrm{F} 1 \mathrm{P})$ to triose phosphates, which subsequently enter the glycolytic/gluconeogenic pathway. Lanaspa and colleagues recently elucidated one of the potential mechanisms by which fructose can cause IHL accumulation. They showed that aldolase B knockout (aldo $\mathrm{B}^{-/-}$) mice displayed a (paradoxical) increase in IHL content, which could be prevented by blocking ketohexokinase. ${ }^{12}$ These observations ascribe a central role for intrahepatic F1P to the pathogenesis of fructose-induced IHL accumulation.

The aim of the present study was to translate these experimental findings to the human situation. For this, we studied 15 aldo $\mathrm{B}^{-/-}$patients (i.e., patients with hereditary fructose intolerance, OMIM\# 229600) in comparison to 15 age-, sexand BMI-matched controls. 


\section{Methods}

\section{Subjects}

In this case-control study, adult aldo $\mathrm{B}^{-/-}$patients were recruited from multiple outpatient metabolic clinics in the Netherlands and Belgium and compared to healthy control subjects matched for age, sex and body mass index (BMI). Diagnosis of hereditary fructose intolerance was confirmed by either a fructose tolerance test $(n=1)$, measurement of aldolase activity in liver biopsy tissue $(n=2)$ or DNA analysis $(n=12)$. Exclusion criteria for this study were contra-indications for magnetic resonance imaging (MRI) or inability to give informed consent.

Participants visited our metabolic ward after an overnight fast of at least eight hours. All completed a health questionnaire regarding (amongst others) habitual alcohol consumption and medical history. Height was determined standing upright against a stadiometer and weight was measured using electronic scales in underwear only. BMI was calculated as weight divided by height squared. Waist circumference was ascertained in standing position with a measuring tape at the level of the umbilicus.

All participants gave written informed consent prior to inclusion in the study. The study was performed according to the Declaration of Helsinki ${ }^{13}$ and approved by the medical ethical committee of Maastricht University Medical Center.

\section{Dietary intake}

Since aldo $\mathrm{B}^{-/-}$patients do not tolerate fructose, they follow a fructose restricted diet. As a consequence, macro- and micronutrient composition of their diet may differ from controls, which in turn may affect the primary outcome of interest. Dietary intake was therefore assessed using a three-day food journal along with a personal interview by the clinical researcher (N.S.). Dietary macro- and micronutrient composition was determined based on the Dutch food composition table. ${ }^{14}$ Daily fructose intake was calculated with a recently developed, extensive sugar composition table ${ }^{15}$, which consists of data from the national Dutch food composition table and internationally available food composition databases (i.e., McCance \& Widdowson's composition of foods integrated data set, UK; the Danish food composition database; and the Finnish food composition database, Fineli). If the fructose content of a specific product was not available, either values of a comparable food product were used or sucrose content was estimated according to total sugar content of the 
specific product. Plasma fatty acid composition was measured as a biomarker of saturated fat intake.

\section{Laboratory measurements}

Glucose tolerance was determined with a standard 75-grams oral glucose tolerance test (OGTT). Participants were instructed to ingest 82.5 grams dextrose monohydrate dissolved in $250 \mathrm{ml}$ water over the course of five minutes, according to the World Health Organization. ${ }^{16}$ Whole blood glucose levels were determined every 15 minutes using the YSI2300 STAT Plus Glucose Lactate Analyzer (YSI, Yellow Springs, OH, USA). Plasma insulin levels were measured at baseline, $t=30, t=60$ and $t=120$ minutes using the Human Insulin Kit from Meso Scale Discovery (Rockville, MD, USA). Subsequently, the area under the curve (AUC) and incremental area under the curve (iAUC) were calculated for blood glucose and plasma insulin excursions. The degree of insulin resistance was estimated with the HOMA2 calculator (www.dtu.ox.ac.uk). The hepatic insulin resistance index (HIRI) was calculated as the square root of the product of the glucose $(\mathrm{mmol} / \mathrm{L})$ and insulin $(\mathrm{pg} / \mathrm{ml})$ AUC during the first 30 minutes of the $\mathrm{OGTT}^{17}$ and expressed as arbitrary units.

At $t=-5$ minutes, i.e., before the ingestion of dextrose monohydrate, blood was drawn for determination of serum uric acid, total cholesterol, HDL cholesterol and triglycerides (Enzymatic colorimetric assay, Cobas 8000 instrument, Roche Diagnostics, Mannheim, Germany). LDL cholesterol was calculated using the Friedewald formula. EDTA-treated blood samples were used for the determination of fatty acid composition and beta-hydroxybutyrate, as described in detail previously. ${ }^{18,19}$

Glycoprofiling of transferrin was carried out using high-resolution mass spectrometry. ${ }^{20}$ In brief, $2 \mu$ of eluted plasma were analyzed on a microfluidic 6540 HPLC-chip-QTOF instrument (Agilent Technologies, California, USA). Data analysis was performed using Agilent Mass Hunter Qualitative Analysis Software B.04.00. The Agilent BioConfirm Software was used to deconvolute the charge distribution raw data to reconstructed mass data. In case there was no monoglycosylated transferrin present in the samples, abundances were estimated at the average reconstructed masses of $77,351 \mathrm{Da}$. These abundances were used to calculate the ratio between monoglycosylated transferrin and diglycosylated transferrin (mono-glyco:di-glyco). 
Visceral adipose tissue area, intrahepatic lipid content and liver stiffness

Visceral adipose tissue area was measured through two-dimensional (2D) T1weighted (T1W) turbo spin echo (TSE) imaging using a 3.0 Tesla Philips Achieva whole body MRI scanner with a sixteen-element torso coil (XL Torso coil, Philips Healthcare, Best, The Netherlands). Ten 5-mm-thick transverse slices with a slice gap of $10 \mathrm{~mm}$ centered at the top of the L5 vertebral body were acquired. Scan parameters were as follows: field of view $=400 \times 322 \mathrm{~mm}$, acquired voxel size $=1.30 \times 1.96 \times 5.00 \mathrm{~mm}$, reconstructed voxel size $=0.78 \times 0.78 \times 5.00 \mathrm{~mm}$, repetition time/echo time $=400 / 10 \mathrm{~ms}$, number of signal averages $=1$, TSE factor $=4$. Visceral adipose tissue area was subsequently calculated offline with the ITK-SNAP software application (http://www.itksnap.org). ${ }^{21}$

IHL content was measured with proton magnetic resonance spectroscopy $\left({ }^{1} \mathrm{H}\right.$ MRS), using the same MRI scanner as for the visceral adipose tissue measurement. Spectra were obtained from a $20 \times 20 \times 20 \mathrm{~mm}$ voxel placed centrally in the lower right lobe of the liver, avoiding large blood vessels and the subcutaneous fat layer. Second-order FASTMAP-based shimming was performed. The following acquisition parameters were used: repetition time/echo time $=4000 / 32.5 \mathrm{~ms}$, data points $=2048$. A total of 32 spectra were acquired with two signal averages per spectrum. In these spectra the water signal was suppressed using frequency-selective pre-pulses. The unsuppressed water signal was acquired as internal reference (8 spectra with 2 signal averages per spectrum). The subject was instructed to breathe exactly at the $4 \mathrm{~s}$-rhythm of the sequence to keep the repetition time constant. Spectra were analyzed as described before. ${ }^{22}$ Briefly, the spectra were fitted using a home-written script in MATLAB R2014b (Mathworks, Natick, MA, USA). The $\mathrm{CH}_{2}$ peak and the unsuppressed water peak were corrected for T2 relaxation. IHL content was expressed as the area percentage of the $\mathrm{CH}_{2}$ peak compared to the water peak.

The degree of liver stiffness, as a proxy for fibrosis ${ }^{23}$, was measured in supine position with transient elastography (FibroScan, Echosens, Paris, France). The standard $\mathrm{M}$ probe was placed in the intercostal region on the lateral side of the right upper abdominal quadrant. A low-frequency elastic shear wave was passed through the liver, after which the velocity (which alters due to different components of the liver) was measured. Subsequently, the shear wave velocity was converted into liver stiffness, expressed in kilopascal (kPa). The median value of ten valid measurements (i.e., interquartile range/median ratio $\leq 30 \%$ ) 
was used for analysis. All measurements were performed by the clinical researcher (N.S.).

\section{Statistical analyses}

Data are expressed as median (interquartile range) and analyzed with a MannWhitney $U$ test. Linear regression analyses were conducted to adjust for differences in dietary intake. The relationships between IHL content, glucose AUC and hypoglycosylated transferrin were analyzed with a Spearman Rank correlation. Results were considered statistically significant at $p<0.05$. All analyses were carried out with the IBM Statistical Package of Social Sciences (SPSS) version 23 for Windows (SPSS inc. Chicago, IL).

\section{Results}

\section{General characteristics and dietary intake}

General characteristics of the fifteen aldo $\mathrm{B}^{-/-}$patients and age-, sex- and BMImatched control subjects are displayed in Table 7.1. Aldo $\mathrm{B}^{-/-}$patients were lean (median BMI $20.4 \mathrm{~kg} / \mathrm{m}^{2}$ ) and consumed significantly less alcohol than controls ( $p=0.04$; Table 7.1). VAT area, HOMA2-IR, uric acid and plasma lipids did not differ between groups (Table 7.1).

Table 7.1 Characteristics of the study population.

\begin{tabular}{lcc}
\hline & Healthy controls & Aldo B $^{-/-}$patients \\
\hline Sex $(\mathrm{M} / \mathrm{F})$ & $11 / 4$ & $11 / 4$ \\
Age $(\mathrm{y})$ & $28(25-52)$ & $31(24-44)$ \\
BMI $\left(\mathrm{kg} / \mathrm{m}^{2}\right)$ & $21.8(21.0-23.3)$ & $20.4(19.3-24.8)$ \\
Waist circumference $(\mathrm{cm})$ & $87(84.3-91.3)$ & $77.5(73.8-93.5)$ \\
VAT $\left(\mathrm{cm}^{3}\right)$ & $264(143-331)$ & $328(119-407)$ \\
Alcohol intake $(\mathrm{U} / \mathrm{day})$ & $0.6(0.1-1.7)$ & $0.1(0.0-0.3)^{*}$ \\
HOMA2-IR & $0.5(0.3-0.6)$ & $0.5(0.4-0.7)$ \\
Total cholesterol $(\mathrm{mmol} / \mathrm{L})$ & $4.1(3.9-5.2)$ & $5.0(4.3-6.2)$ \\
HDL cholesterol $(\mathrm{mmol} / \mathrm{L})$ & $1.4(1.1-1.8)$ & $1.6(1.4-2.3)$ \\
LDL cholesterol $(\mathrm{mmol} / \mathrm{L})$ & $2.4(1.8-3.3)$ & $3.1(2.0-4.2)$ \\
Triglycerides $(\mathrm{mmol} / \mathrm{L})$ & $0.9(0.5-1.2)$ & $0.8(0.7-1.3)$ \\
Uric acid $(\mathrm{mmol} / \mathrm{L})$ & $0.33(0.28-0.38)$ & $0.30(0.23-0.34)$ \\
\hline
\end{tabular}

Data are expressed as median (interquartile range). ${ }^{*} p<0.05$ versus controls, analyzed with a Mann-Whitney $U$ test. For technical reasons VAT area estimates could only be obtained for 14 aldo $\mathrm{B}^{-/}$patients and 10 controls. Abbreviations: body mass index (BMI), visceral adipose tissue (VAT), Homeostasis Model Assessment 2 Insulin Resistance (HOMA2-IR), high-density lipoprotein (HDL), low-density lipoprotein (LDL).

As expected, dietary fructose intake was very low in aldo $\mathrm{B}^{-/-}$patients $(p<0.0001$ versus controls; Figure 7.1, panel A). Total caloric intake was 
nevertheless not different between aldo $B^{-/}$patients and controls $(p=0.93$; Figure 7.1, panel B). Of all macronutrients, aldo $\mathrm{B}^{-1-}$ patients consumed significantly more protein $(p=0.005$; Figure 7.1 , panel $C)$ and saturated fat $(p=0.026$; Figure 7.1, panel D). Fatty acid composition measurement of plasma triglycerides, cholesteryl esters and phospholipids confirmed the higher saturated fat intake, in particular dairy products, as noted by a higher proportion of $\mathrm{C} 14: 0$ in all lipid fractions and a higher $\mathrm{C} 15: 0$ in the cholesteryl ester and phospholipid fractions (Figure 7.2, panel A-C). Total fat and carbohydrate intake were not significantly different between groups $(p=0.15$; Figure 7.1, panel $E$ and $p=0.11$; Figure 7.1, panel F, respectively).
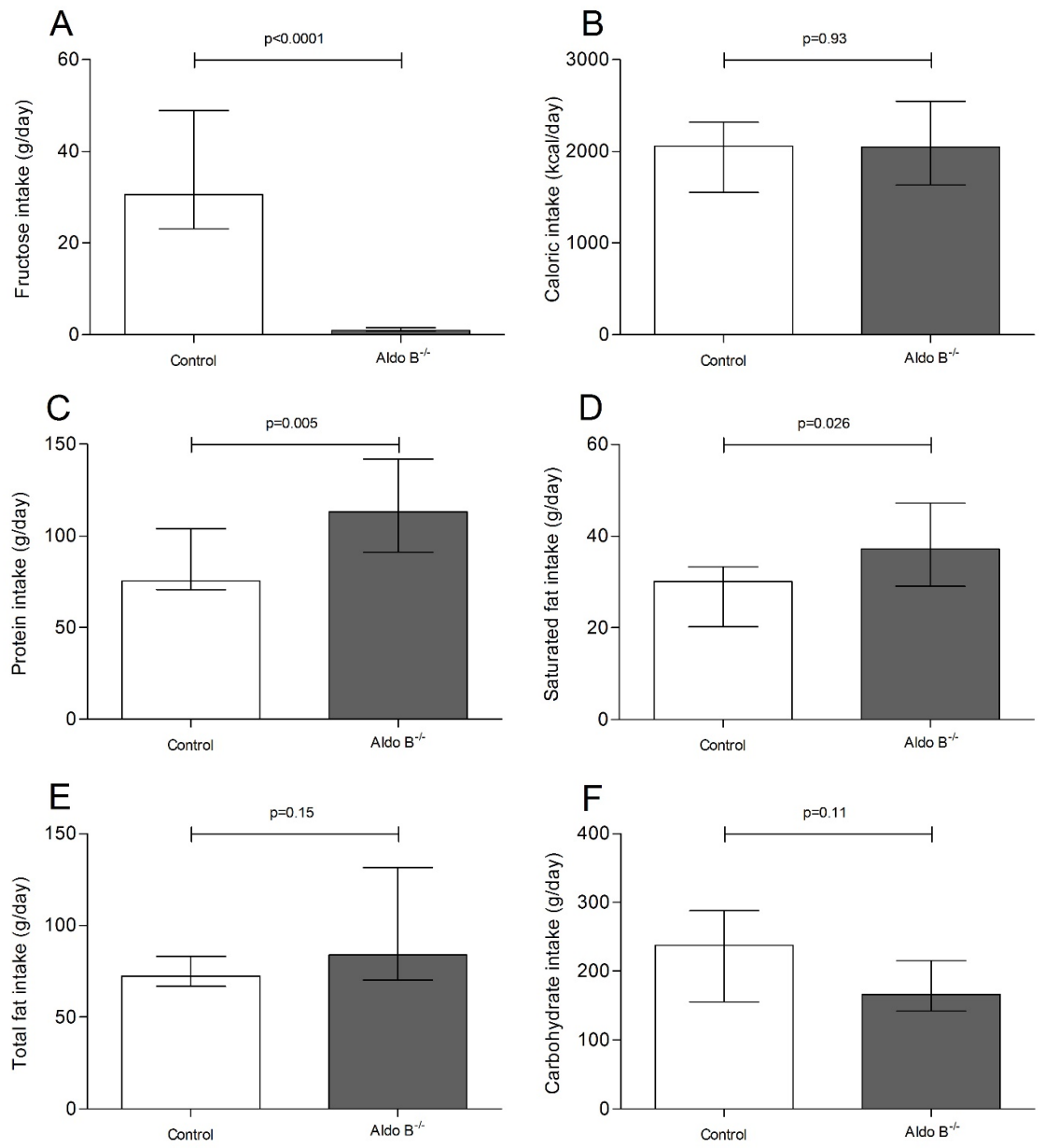

Figure 7.1 Dietary intake. Daily intake of fructose (A), total calories (B), protein (C), saturated fat (D), total fat $(\mathrm{E})$ and carbohydrates $(\mathrm{F})$ in aldo $\mathrm{B}^{-/-}$patients (grey bars) and controls (white bars). Data are expressed as median (interquartile range). Analyzed with a Mann-Whitney $U$ test. 

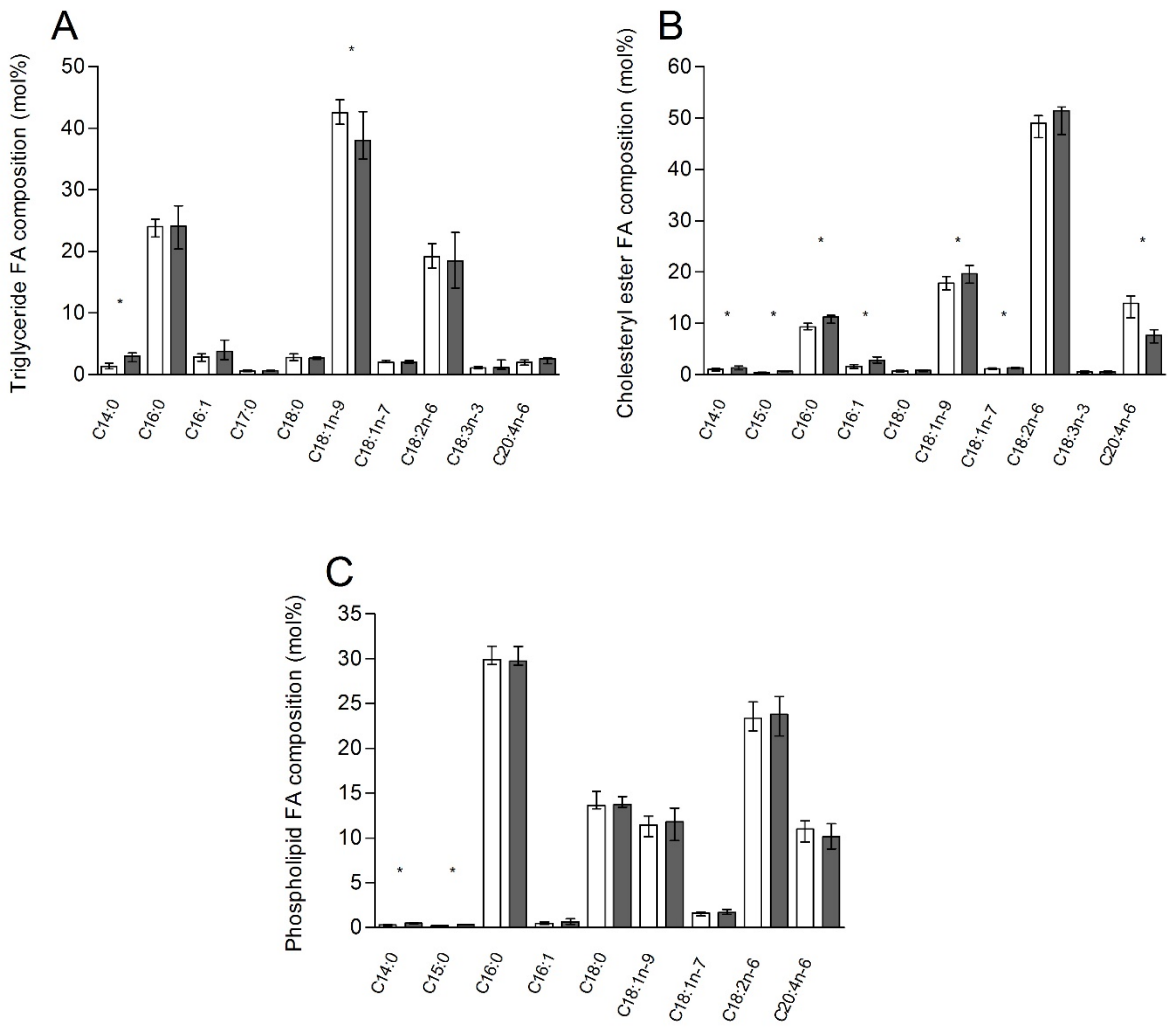

Figure 7.2 Plasma fatty acid composition as a biomarker of saturated fat intake. Fatty acid composition of plasma triglycerides (A), cholesteryl esters (B) and phospholipids $(C)$ in aldo $B^{-/-}$patients (grey bars) and controls (white bars). ${ }^{*} p<0.05$ versus controls. Data are expressed as median (interquartile range). Analyzed with a Mann-Whitney $U$ test.

\section{Intrahepatic lipid content and glucose tolerance}

$\mathrm{IHL}$ content was higher in aldo $\mathrm{B}^{-/-}$patients than controls $(\mathrm{p}=0.001$; Figure 7.3 , panel A). The prevalence of hepatic steatosis in aldo $B^{-1-}$ was $3 / 15(20 \%)$ and $5 / 15(33 \%)$, depending on the population-based and pathology-based cut-off values of $5.6 \%$ and $3.0 \%$, respectively. ${ }^{24,25}$ Only one control individual had an $\mathrm{IHL}$ content that was greater than $3.0 \%$. Statistical adjustment for saturated fat or protein intakes did not affect the difference in IHL content between aldo $\mathrm{B}^{-1-}$ patients and controls $(p=0.005$ and $p=0.03$, respectively, for the adjusted difference in $\mathrm{IHL}$ content). Liver stiffness did not differ between aldo $\mathrm{B}^{-/}$ patients and controls $(p=0.098$; Figure 7.3 , panel $B)$. One out of 15 aldo $B^{-1-}$ patients $(7 \%)$ had a high liver stiffness measurement $(9.0 \mathrm{kPa})$, corresponding with fibrosis stage 3 or higher. ${ }^{26}$ 
Plasma glucose excursions were higher in aldo $\mathrm{B}^{-/-}$patients during a standard 75-grams OGTT (Figure 7.3, panel C). Consequently, both the AUC and iAUC were greater in aldo $B^{-1-}$ patients $(p=0.043$ and $p=0.034$, respectively). IHL content correlated significantly with glucose AUC and iAUC in the overall population, i.e., aldo $B^{-/-}$and controls combined $\left(r_{s}: 0.42, p=0.025\right.$ and $r_{s}: 0.45$, $p=0.021$, respectively). Plasma insulin excursions appeared somewhat higher at all time points in aldo $B^{-/ 2}$ patients (Figure 7.3, panel $D$ ), but were not statistically different ( $p=0.49$ and $p=0.65$ for the AUC and iAUC, respectively). The HIRI did not differ between both groups (920 [interquartile range: 753-1320] in aldo $B^{-/-}$patients versus 700 [interquartile range: 582-1330] in controls, $p=0.29$ ).
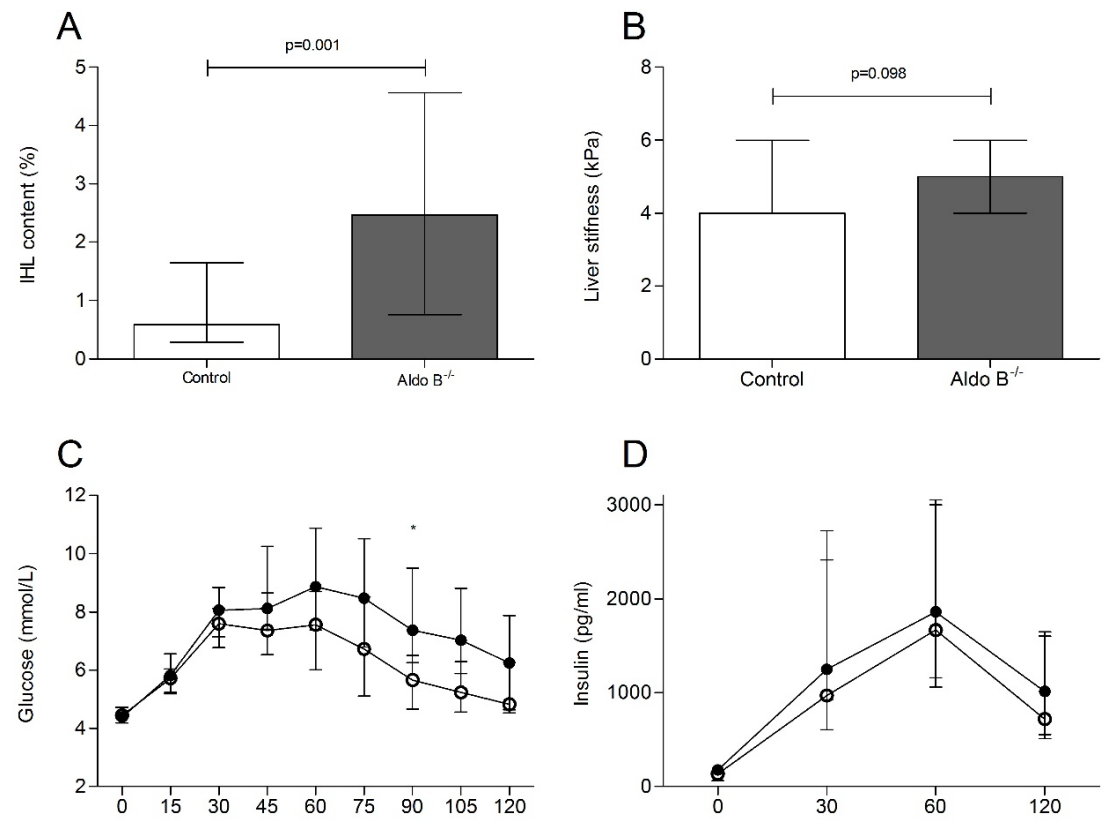

Figure 7.3 Intrahepatic lipid content, liver stiffness and oral glucose tolerance test. Intrahepatic lipid $(\mathrm{IHL})$ content $(\mathrm{A})$, liver stiffness $(\mathrm{B})$ and glucose and insulin excursions during an oral glucose tolerance test (OGTT) (C-D) in aldo $\mathrm{B}^{-/-}$patients (grey bars/solid circles) and controls (white bars/open circles). Data are expressed as median (interquartile range). ${ }^{*} p<0.05$ versus controls. Analyzed with a Mann-Whitney $U$ test.

\section{Hypoglycosylated transferrin and beta-hydroxybutyrate levels}

To gain more insight in the pathogenesis of the increased IHL content in aldo $\mathrm{B}^{-/}$patients, we measured glycosylation of transferrin as a measure of intrahepatic F1P concentrations. Previous studies have shown that untreated 
aldo $\mathrm{B}^{-/-}$patients have an abnormal transferrin glycosylation pattern ${ }^{27,28}$ as a consequence of F1P-mediated competitive inhibition of mannose phosphate isomerase (MPI) (Figure 7.4, panel A). ${ }^{29}$ Hypoglycosylated transferrin (expressed as the ratio of monoglycosylated transferrin divided by the intact glycoprotein, i.e., diglycosylated transferrin) was also more abundant in aldo $\mathrm{B}^{-/-}$patients on a fructose restricted diet $(p<0.001$ versus controls; Figure 7.4, panel B). Hypoglycosylated transferrin levels correlated with IHL content, not with glucose $A \cup C$, in the overall population $\left(r_{s}: 0.56, p=0.002\right.$ and $r_{s}: 0.31$, $\mathrm{p}=0.11$, respectively).

We subsequently measured plasma beta-hydroxybutyrate levels, a biomarker of hepatic fatty acid oxidation, and observed significantly lower levels in aldo $\mathrm{B}^{-/-}$patients than in controls $(p=0.009$; Figure 7.4 , panel $C)$.

A
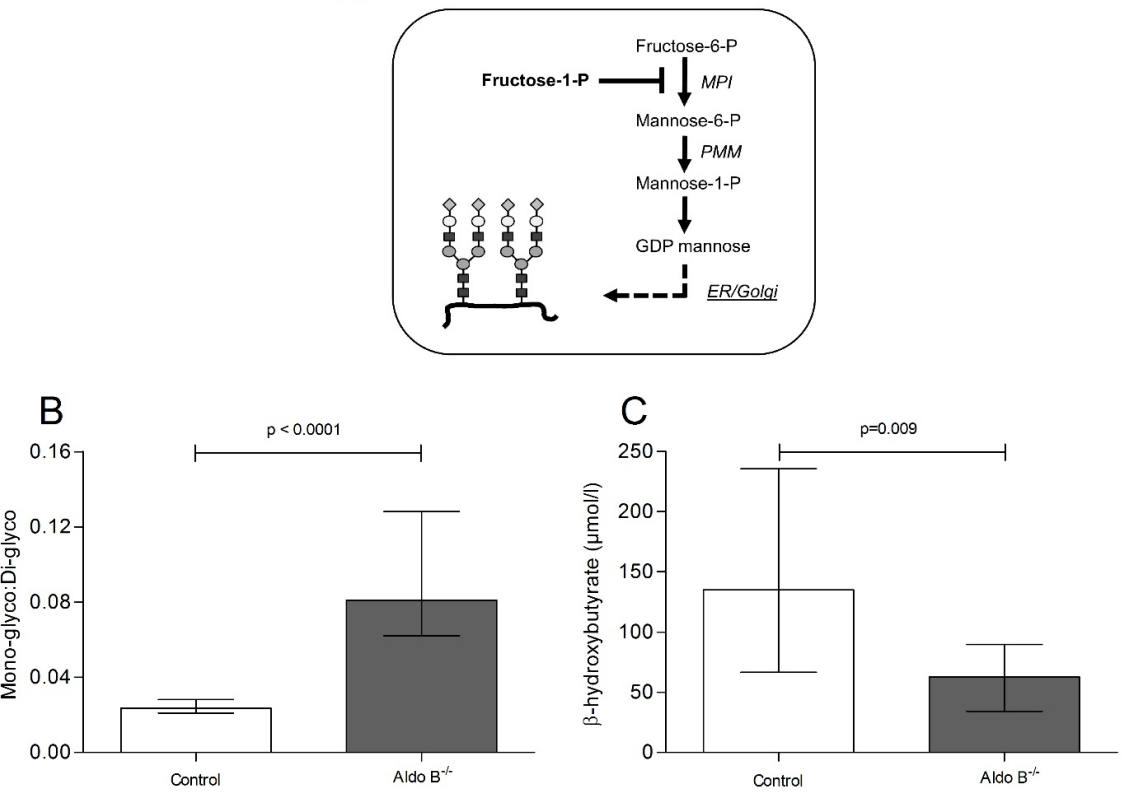

Figure 7.4 Hypoglycosylated transferrin and beta-hydroxybutyrate levels. Normal transferrin consists of two sialylated, biantennary complexes attached to the glycosylation sites of the protein. Suppression of transferrin glycosylation, via the competitive inhibition of mannose phosphate isomerase (MPI) by fructose-1-phosphate (fructose-1-P), results in the loss of one or both antennary structures, i.e., more monoglycosylated transferrin (A). Monoglycosylated transferrin/diglycosylated transferrin $(B)$ and beta-hydroxybutyrate $(C)$ in aldo $\mathrm{B}^{-/-}$patients (grey bars) and controls (white bars). Data are expressed as median (interquartile range). Analyzed with a Mann-Whitney $U$ test. Abbreviations: fructose-6-phosphate (fructose-6-P), mannose-6-phosphate (mannose-6-P), phosphomannomutase (PMM), guanosine diphosphate (GDP), endoplasmic reticulum $(E R)$, monoglycosylated transferrin/diglycosylated transferrin (mono-glyco:di-glyco). 


\section{Discussion}

The contribution of dietary fructose to the development and progression of NAFLD is still under debate, mainly because the exact mechanism by which fructose leads to $\mathrm{IHL}$ accumulation has not been fully elucidated. In the present study we showed that aldo $\mathrm{B}^{-/-}$patients, who are characterized by an impaired breakdown of $\mathrm{F} 1 \mathrm{P}$, displayed a higher $\mathrm{IHL}$ content compared to control subjects. This was accompanied by a decrease in glucose tolerance.

In the present study, aldo $\mathrm{B}^{-/}$patients were matched to healthy controls with respect to age, sex and $\mathrm{BMI}$, the latter being one of the principal determinants of $\mathrm{IHL}$ content. ${ }^{30}$ Since aldo $\mathrm{B}^{-/-}$patients do not tolerate fructose, it was inevitable that there would be differences in dietary intakes between the groups. Previous studies have shown that short-term changes in dietary intake can affect IHL content. ${ }^{22,31}$ Three-days food journals prior to the measurements revealed that aldo $\mathrm{B}^{-/-}$patients consumed less alcohol-containing beverages and more saturated fat and protein. A recently published intervention study reported that overfeeding obese individuals with saturated fat increased $\mathrm{IHL}$ to a greater extent than overfeeding unsaturated fat or simple sugars. ${ }^{4}$ In contrast, a six-week high protein diet (30\% of total energy) that was rich in meat and dairy foods significantly decreased IHL content in patients with type 2 diabetes. ${ }^{32}$ Interestingly, Bartolotti and colleagues showed that the effects of a high-saturated fat diet on IHL content was blunted when this diet was combined with a high protein diet. ${ }^{33}$ In the present study, there are several arguments that corroborate the suggestion that dietary differences do not entirely account for the observed differences in IHL content between aldo $\mathrm{B}^{-/}$patients and controls. Firstly, alcohol intake was low in both groups (median intake: $0.1 \mathrm{U} /$ day versus $0.6 \mathrm{U} /$ day in aldo $\mathrm{B}^{-/-}$patients and controls, respectively) and its potential effect on IHL content, if any, would minimize the difference in IHL content between both groups. Second, statistical adjustment for saturated fat or protein intake did not affect the outcomes.

The results of the present study in aldo $\mathrm{B}^{-/}$patients chronically treated with a fructose restricted diet (containing only small amounts of fructose) are in agreement with the outcomes of a recent experimental study in aldo $\mathrm{B}^{-{ }^{-}}$mice chronically exposed to small amounts of fructose in the chow $(\sim 0.3 \%)$. Analogous to our ${ }^{1} \mathrm{H}$-MRS findings and the increased proportion of hypoglycosylated transferrin (used as plasma biomarker of intrahepatic F1P) in aldo $\mathrm{B}^{-/-}$patients, aldo $\mathrm{B}^{-/-}$mice were also characterized by a greater $\mathrm{IHL}$ content and increased intrahepatic F1P levels. ${ }^{12}$ Subsequent detailed examination of the potential pathways involved in the pathogenesis of IHL 
accumulation revealed upregulation of de novo lipogenesis genes and increased cytosolic expression of glucokinase in aldo $\mathrm{B}^{-/}$mouse livers. ${ }^{12}$ Interestingly, intrahepatic F1P is a potent disruptor of the nuclear glucokinaseglucokinase regulatory protein complex, which causes migration of glucokinase towards the cytosolic space where it facilitates the conversion of glucose to glucose-6-phosphate, the first step in glycolysis and subsequent de novo lipogenesis. ${ }^{34}$ Although the present study was not specifically designed to elucidate the exact mechanism by which aldo $\mathrm{B}^{-/-}$causes $\mathrm{IHL}$ accumulation in humans, we observed reduced serum beta-hydroxybutyrate levels in aldo $\mathrm{B}^{-/-}$ patients, used as a biomarker of hepatic fatty acid beta-oxidation (and indirectly of de novo lipogenesis, which is inversely linked to beta-oxidation ${ }^{35,36}$ ).

In the current study, aldo $\mathrm{B}^{-/}$patients were more glucose intolerant than age-, sex- and BMI-matched controls, which is generally explained by either beta cell insufficiency or insulin resistance. Although Gerst et al. recently demonstrated that aldolase $B$ is also involved in pancreatic islet function, their results suggested that higher aldolase $B$ activity is associated with reduced insulin secretion, which appears to be in contrast to our findings. Previous studies have shown that $\mathrm{IHL}$ content is positively associated with endogenous glucose production ${ }^{37,38}$, which is line with the positive association between IHL content and the (i)AUC for glucose in the current study. However, indices of hepatic insulin resistance, such as HOMA2-IR and $\mathrm{HIRI}^{17}$, were not significantly different between aldo $\mathrm{B}^{-1-}$ patients and controls. Whether this is the consequence of a lack of statistical power, or truly indicative of a dissociation between $\mathrm{IHL}$ and insulin resistance, as observed in other geneticallydetermined conditions ${ }^{39,40}$, remains to be elucidated.

Our study has several strengths and limitations. Although hereditary fructose intolerance is a rare inborn error of metabolism (estimated prevalence between 1 in 23,000 to 31,000 people $^{41-43}$ ), we were able to include a unique sample of carefully phenotyped aldo $\mathrm{B}^{-/}$patients. Second, as already mentioned, this study was not specifically designed to unravel the underlying mechanism by which aldolase B deficiency causes IHL accumulation in humans. For this, future studies using stable isotopes to assess de novo lipogenesis are required. Finally, dietary intakes were assessed with a three-day food journal and personal interview by the clinical researcher, which could be subjective and susceptible for underreporting. ${ }^{44}$ However, we used an objective biomarker for some aspects of dietary intake (i.e., fatty acid composition ${ }^{45}$ ) that confirmed the results from food journals. 
In conclusion, the present study extends previous observations in aldo $\mathrm{B}^{-/-}$mice by showing that aldo $\mathrm{B}^{-/-}$patients are also characterized by an increased $\mathrm{IHL}$ content. Our findings suggest that the accumulation of intermediates of fructolysis, i.e., F1P, can cause IHL accumulation via impaired beta-oxidation. 


\section{Literature}

1. Sayiner M, Koenig A, Henry L, Younossi ZM. Epidemiology of Nonalcoholic Fatty Liver Disease and Nonalcoholic Steatohepatitis in the United States and the Rest of the World. Clin Liver Dis 2016;20:205-14.

2. Armstrong MJ, Adams LA, Canbay A, Syn WK. Extrahepatic complications of nonalcoholic fatty liver disease. Hepatology 2014;59:1174-97.

3. Haas JT, Francque S, Staels B. Pathophysiology and Mechanisms of Nonalcoholic Fatty Liver Disease. Annu Rev Physiol 2016;78:181-205.

4. Luukkonen PK, Sadevirta S, Zhou Y, et al. Saturated Fat Is More Metabolically Harmful for the Human Liver Than Unsaturated Fat or Simple Sugars. Diabetes Care 2018;41:1732-9.

5. McGuinness OP, Cherrington AD. Effects of fructose on hepatic glucose metabolism. Curr Opin Clin Nutr Metab Care 2003;6:441-8.

6. Kanerva N, Sandboge S, Kaartinen NE, Mannisto S, Eriksson JG. Higher fructose intake is inversely associated with risk of nonalcoholic fatty liver disease in older Finnish adults. Am J Clin Nutr 2014;100:1133-8.

7. Wehmeyer MH, Zyriax BC, Jagemann B, et al. Nonalcoholic fatty liver disease is associated with excessive calorie intake rather than a distinctive dietary pattern. Medicine 2016;95: e3887.

8. Johnston RD, Stephenson MC, Crossland $\mathrm{H}$, et al. No difference between high-fructose and high-glucose diets on liver triacylglycerol or biochemistry in healthy overweight men. Gastroenterology 2013;145:1016-25 e2.

9. Chiu S, Sievenpiper JL, de Souza RJ, et al. Effect of fructose on markers of non-alcoholic fatty liver disease (NAFLD): a systematic review and meta-analysis of controlled feeding trials. Eur J Clin Nutr 2014;68:416-23.

10. Stanhope KL, Schwarz JM, Keim NL, et al. Consuming fructose-sweetened, not glucosesweetened, beverages increases visceral adiposity and lipids and decreases insulin sensitivity in overweight/obese humans. J Clin Invest 2009;119:1322-34.

11. Silbernagel G, Machann J, Unmuth S, et al. Effects of 4-week very-high-fructose/glucose diets on insulin sensitivity, visceral fat and intrahepatic lipids: an exploratory trial. Br J Nutr 2011;106:79-86.

12. Lanaspa MA, Andres-Hernando A, Orlicky DJ, et al. Ketohexokinase $C$ blockade ameliorates fructose-induced metabolic dysfunction in fructose-sensitive mice. J Clin Invest 2018;128: 2226-38.

13. World Medical Association Declaration of Helsinki: ethical principles for medical research involving human subjects. JAMA 2013;310:2191-4.

14. NEVO-tabel; Nederlands Voedingsstoffenbestand 2011 RV, Den Haag. 2011.

15. Sluik D, Engelen Al, Feskens EJ. Fructose consumption in the Netherlands: the Dutch National Food Consumption Survey 2007-2010. Eur J Clin Nutr 2015;69:475-81.

16. WHO. Definition, Diagnosis and Classification of Diabetes Mellitus and its Complications. Geneva: World Health Organisation; 1999.

17. Abdul-Ghani MA, Matsuda M, Balas B, DeFronzo RA. Muscle and liver insulin resistance indexes derived from the oral glucose tolerance test. Diabetes Care 2007;30:89-94.

18. Hodson L, Bickerton AS, McQuaid SE, et al. The contribution of splanchnic fat to VLDL triglyceride is greater in insulin-resistant than insulin-sensitive men and women: studies in the postprandial state. Diabetes 2007;56:2433-41.

19. Hodson L, McQuaid SE, Karpe F, Frayn KN, Fielding BA. Differences in partitioning of meal fatty acids into blood lipid fractions: a comparison of linoleate, oleate, and palmitate. Am J Physiol Endocrinol Metab 2009;296:E64-71.

20. van Scherpenzeel M, Steenbergen G, Morava E, Wevers RA, Lefeber DJ. High-resolution mass spectrometry glycoprofiling of intact transferrin for diagnosis and subtype identification in the congenital disorders of glycosylation. Transl Res 2015;166:639-49 e1. 
21. Yushkevich PA, Piven J, Hazlett HC, et al. User-guided 3D active contour segmentation of anatomical structures: significantly improved efficiency and reliability. Neuroimage 2006;31:1116-28.

22. Lindeboom L, Nabuurs $\mathrm{Cl}$, Hesselink MK, Wildberger JE, Schrauwen P, SchrauwenHinderling VB. Proton magnetic resonance spectroscopy reveals increased hepatic lipid content after a single high-fat meal with no additional modulation by added protein. Am J Clin Nutr 2015;101:65-71.

23. Cassinotto $\mathrm{C}$, Boursier $\mathrm{J}$, de Ledinghen $\mathrm{V}$, et al. Liver stiffness in nonalcoholic fatty liver disease: A comparison of supersonic shear imaging, FibroScan, and ARFI with liver biopsy. Hepatology 2016;63:1817-27.

24. Nasr P, Forsgren MF, Ignatova S, et al. Using a 3\% Proton Density Fat Fraction as a Cut-Off Value Increases Sensitivity of Detection of Hepatic Steatosis, Based on Results From Histopathology Analysis. Gastroenterology 2017;153:53-5 e7.

25. Szczepaniak LS, Nurenberg P, Leonard D, et al. Magnetic resonance spectroscopy to measure hepatic triglyceride content: prevalence of hepatic steatosis in the general population. Am J Physiol Endocrinol Metab 2005;288:E462-8.

26. Wong VW, Vergniol J, Wong GL, et al. Diagnosis of fibrosis and cirrhosis using liver stiffness measurement in nonalcoholic fatty liver disease. Hepatology 2010;51:454-62.

27. Adamowicz M, Ploski R, Rokicki $D$, et al. Transferrin hypoglycosylation in hereditary fructose intolerance: using the clues and avoiding the pitfalls. J Inherit Metab Dis 2007;30:407.

28. Quintana E, Sturiale L, Montero R, et al. Secondary disorders of glycosylation in inborn errors of fructose metabolism. J Inherit Metab Dis 2009;32 Suppl 1:S273-8.

29. Jaeken J, Pirard M, Adamowicz M, Pronicka E, van Schaftingen E. Inhibition of phosphomannose isomerase by fructose 1-phosphate: an explanation for defective $\mathrm{N}$ glycosylation in hereditary fructose intolerance. Pediatric Res 1996;40:764-6.

30. EASL-EASD-EASO Clinical Practice Guidelines for the management of non-alcoholic fatty liver disease. Diabetologia 2016;59:1121-40.

31. Bawden S, Stephenson M, Falcone $\mathrm{Y}$, et al. Increased liver fat and glycogen stores after consumption of high versus low glycaemic index food: A randomized crossover study. Diabetes Obes Metab 2017;19:70-7.

32. Markova M, Pivovarova O, Hornemann S, et al. Isocaloric Diets High in Animal or Plant Protein Reduce Liver Fat and Inflammation in Individuals With Type 2 Diabetes. Gastroenterology 2017;152:571-85.e8.

33. Bortolotti M, Kreis R, Debard C, et al. High protein intake reduces intrahepatocellular lipid deposition in humans. Am J Clin Nutr 2009;90:1002-10.

34. van Schaftingen E, Vandercammen A, Detheux M, Davies DR. The regulatory protein of liver glucokinase. Adv Enzyme Regul 1992;32:133-48.

35. McGarry JD, Mannaerts GP, Foster DW: A possible role for malonyl-CoA in the regulation of hepatic fatty acid oxidation and ketogenesis. J Clin Invest 1977;60:265-270

36. Abu-Elheiga L, Brinkley WR, Zhong L, et al.: The subcellular localization of acetyl-CoA carboxylase 2. Proc Natl Acad Sci U S A 2000;97:1444-1449

37. Kelley DE, McKolanis TM, Hegazi RA, Kuller LH, Kalhan SC. Fatty liver in type 2 diabetes mellitus: relation to regional adiposity, fatty acids, and insulin resistance. Am J Physiol Endocrinol Metab 2003;285:E906-16.

38. Seppala-Lindroos A, Vehkavaara S, Hakkinen AM, et al. Fat accumulation in the liver is associated with defects in insulin suppression of glucose production and serum free fatty acids independent of obesity in normal men. J Clin Endocrinol Metab 2002;87:3023-8.

39. Visser ME, Lammers NM, Nederveen AJ, et al. Hepatic steatosis does not cause insulin resistance in people with familial hypobetalipoproteinaemia. Diabetologia 2011;54:2113-21.

40. Amaro A, Fabbrini E, Kars M, et al. Dissociation between intrahepatic triglyceride content and insulin resistance in familial hypobetalipoproteinemia. Gastroenterology 2010;139:149-53. 
41. James CL, Rellos $P$, Ali M, Heeley AF, Cox TM. Neonatal screening for hereditary fructose intolerance: frequency of the most common mutant aldolase B allele (A149P) in the British population. J Med Genet 1996;33:837-41.

42. Santer R, Rischewski J, von Weihe M, et al. The spectrum of aldolase B (ALDOB) mutations and the prevalence of hereditary fructose intolerance in Central Europe. Hum Mutat 2005; 25:594.

43. Gruchota J, Pronicka E, Korniszewski L, et al. Aldolase B mutations and prevalence of hereditary fructose intolerance in a Polish population. Mol Genet Metab 2006;87:376-8.

44. Shim JS, Oh K, Kim HC. Dietary assessment methods in epidemiologic studies. Epidemiol Health 2014;36:e2014009.

45. Hodson L, Skeaff CM, Fielding BA. Fatty acid composition of adipose tissue and blood in humans and its use as a biomarker of dietary intake. Prog Lipid Res 2008;47:348-80. 



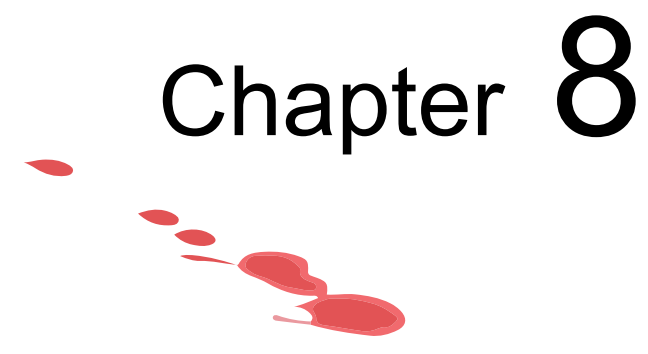

Kidney and vascular function in hereditary fructose intolerance
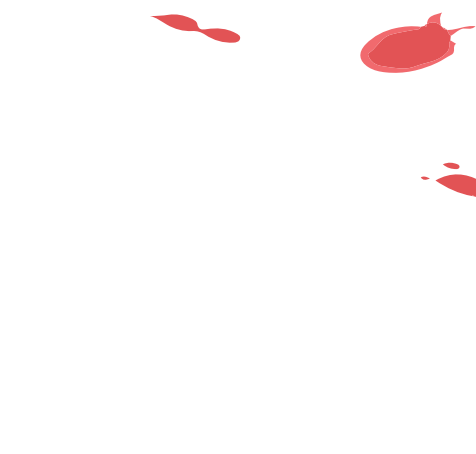

Kidney and vascular function in adult patients with hereditary fructose intolerance

Nynke Simons, François-Guillaume Debray, Nicolaas C. Schaper, Edith J.M. Feskens, Carla E.M. Hollak, Judith A.P. Bons, Jörgen Bierau, Alfons J.H.M. Houben, Casper G. Schalkwijk, Coen D.A. Stehouwer, David Cassiman, Martijn C.G.J. Brouwers 


\section{Abstract}

\section{Background}

Previous studies have shown that patients with hereditary fructose intolerance (HFI) are characterized by a greater intrahepatic lipid content, despite a fructose-restricted diet. The present study aimed to examine the long-term consequences of HFI on other aldolase-B-expressing organs, i.e., the kidney and vascular endothelium.

\section{Methods}

Fifteen adult HFI patients were compared to healthy control individuals matched for age, sex and body mass index. Aortic stiffness was assessed by carotid-femoral pulse wave velocity (cf-PWV) and endothelial function by peripheral arterial tonometry, skin laser doppler flowmetry and the endothelial function biomarkers soluble E-selectin [sE-selectin] and von Willebrand factor. Serum creatinine and cystatin $\mathrm{C}$ were measured to estimate the glomerular filtration rate (eGFR). Urinary glucose and amino acid excretion and the ratio of tubular maximum reabsorption of phosphate to GFR (TmP/GFR) were determined as measures of proximal tubular function.

\section{Results}

Median systolic blood pressure was significantly higher in HFI patients (127 versus $122 \mathrm{mmHg}, p=0.045)$. Pulse pressure and cf-PWV did not differ between the groups ( $p=0.37$ and $p=0.49$, respectively). Of all endothelial function markers, only sE-selectin was significantly higher in HFI patients $(p=0.004)$. eGFR was significantly higher in HFI patients than healthy controls (119 versus $104 \mathrm{ml} / \mathrm{min} / 1.73 \mathrm{~m}^{2}, p=0.001$, respectively). All measurements of proximal tubular function did not differ significantly between the groups.

\section{Conclusions}

Adult HFI patients treated with a fructose-restricted diet are characterized by a higher sE-selectin level and slightly higher systolic blood pressure, which in time could contribute to a greater cardiovascular risk. The exact cause and, hence, clinical consequences of the higher eGFR in HFI patients, deserves further study. 


\section{Background}

Hereditary fructose intolerance (HFI; OMIM\# 229600) is an autosomal recessive metabolic disorder ${ }^{1}$ with an estimated incidence of 1 in every 20,000 newborns. ${ }^{2} \mathrm{HFI}$ is caused by mutations in the gene encoding aldolase $\mathrm{B}$ $(A L D O B)$ that is predominantly expressed in liver, kidney and small intestine. ${ }^{3}$ Aldolase $B$ (EC 4.1.2.13) catalyzes the conversion of fructose 1,6-bisphosphate and fructose 1-phosphate to glyceraldehyde(-3-phosphate) and dihydroxyacetone phosphate. ${ }^{4}$ Fructose ingestion in HFI patients causes accumulation of fructose 1-phosphate and intracellular phosphate and ATP depletion, resulting in acute and chronic cellular dysfunction. In the short term, it produces nausea, vomiting, abdominal pain and hypoglycemia. Chronic exposure to fructose can result in cirrhosis, liver failure, generalized proximal tubular dysfunction (i.e., Fanconi syndrome), growth retardation or even death..$^{5-9}$

Treatment of HFI consists of adherence to a diet that is devoid of fructose. ${ }^{10}$ Although this diet is adequate in providing a relatively normal, symptom-free life, little is known about the long-term consequences of HFI and its related diet.

We and others recently showed that HFI patients treated with a fructoserestricted diet are characterized by a higher intrahepatic lipid content ${ }^{11-13}$ and glucose intolerance compared to healthy controls. ${ }^{11}$ In addition, one out of $15 \mathrm{HFI}$ patients had clinically relevant liver fibrosis. ${ }^{11}$

To date, the long-term effects of HFI on other aldolase-B-expressing organs, such as the kidney, have not been described. Experimental studies have shown that aldolase $B$ is also expressed in endothelial cells ${ }^{14}$, vascular smooth muscle cells and aorta ${ }^{15,16}$, and is actively involved in vascular remodeling. ${ }^{15}$

Therefore, the aim of the present study was to investigate blood pressure, aortic stiffness, endothelial function, glomerular function and proximal tubular function in adult HFI patients treated with a fructose-restricted diet.

\section{Methods}

\section{Study population}

Details of this study have recently been described. ${ }^{11}$ Briefly, in this case-control study patients with a confirmed diagnosis of $\mathrm{HFI}$ (either via a fructose tolerance 
test, measurement of aldolase B activity in liver biopsy tissue or DNA analysis) were recruited from several outpatient metabolic clinics in the Netherlands and Belgium and compared to healthy control individuals matched for age, sex and BMI. Exclusion criteria for participation were contra-indications for magnetic resonance imaging (the quantification of intrahepatic lipid content was the primary outcome measurement of this study ${ }^{11}$ ) or inability to give informed consent. Participants visited the metabolic ward after an overnight fast of at least eight hours. All measurements were performed in the fasting state. All participants gave written informed consent prior to inclusion in the study. The study was performed according to the Declaration of Helsinki ${ }^{17}$ and approved by the medical ethical committee of Maastricht University Medical Center.

\section{Measurements of outcomes}

Blood pressure, pulse wave velocity and endothelial function

Measurement of systolic and diastolic blood pressure was done twice in sitting position on the right upper arm after 10 minutes of rest with 3 minutes interval (Omron, Hoofddorp, The Netherlands). The mean of the two measurements was used for further analysis. Pulse pressure was calculated as the difference between systolic and diastolic blood pressure.

Aortic stiffness - i.e., the cushioning capacity of the aorta, which is a determinant of systolic and diastolic blood pressure and, hence, a cardiovascular risk factor ${ }^{18}$ - was estimated by carotid-femoral pulse wave velocity (cf-PWV) using the SphygmoCor device (AtCor Medical, Sydney, Australia), which is based on the speed of travel of a pulse along an arterial segment. $^{19}$ All measurements were performed in the supine position after 10 minutes of rest. The median of at least three adequate measurements was used for statistical analysis.

The function of the endothelium - the vascular barrier that regulates the availability of nitric oxide (and, hence, vascular tone), permeability, leukocyte adhesion and procoagulant activity ${ }^{20}$ - was assessed by several function tests (peripheral arterial tonometry and skin laser doppler flowmetry) and serum biomarkers (soluble E-selectin and von Willebrand). First, we performed peripheral arterial tonometry (RH-PAT; ENDOPAT Itamar Medical, Caesarea, Israel), which measures digital pulsatile arterial pressure changes accompanying pulse waves during reactive hyperemia after local ischemia. ${ }^{21}$ For this, a blood pressure cuff was placed on the right upper arm and a $\mathrm{RH}$ PAT probe, consisting of two finger-mounted probes, was placed on one finger 
(digitus III) of both the right (test) and left (control) hand. After an equilibration period of 5 minutes, the blood pressure cuff was inflated to a suprasystolic pressure for 5 minutes. The cuff was subsequently deflated and RH-PAT recording was proceeded for another 5 minutes. The reactive hyperemia index $(\mathrm{RHI})$ was calculated as the ratio of the relative change in PAT signal amplitude in the test arm (= the average PAT signal amplitude during reactive hyperemia divided by the average PAT signal amplitude during baseline) over the control arm. Lower $\mathrm{RHI}$ indicates a diminished post-occlusion hyperemia, which reflects endothelial dysfunction of the small arteries.

Second, we performed skin laser doppler flowmetry (LDF) using a laserDoppler system (Periflux 5000, Perimed, Järfalla, Sweden) accoutered with a thermostatic laser-Doppler probe (PF457; Perimed), which measures changes in microcirculatory blood flow on the dorsal side of the left lower arm during local heating, using changes in the wavelength imparted by moving blood cells to the probing light. ${ }^{22}$ All measurements were performed in a climate-controlled room at $24^{\circ} \mathrm{C}$. $^{23}$ The percentage increase of the skin blood flow (measured in perfusion units $(\mathrm{PU})$ ) during local heating over the baseline skin blood flow was used to quantify the heat-induced skin hyperemic response. Lower heatinduced skin hyperemia reflects microvascular endothelial dysfunction. ${ }^{24}$

In addition to RH-PAT and LDF, plasma soluble E-selectin (sE-selectin; an endothelium-specific marker that facilitates leucocyte adhesion) and von Willebrand factor (VWF; an endothelial marker that regulates coagulation) were measured using a Diaclone ELISA kit (Diaclone SAS, Besançon Cedex, France) and Sandwich ELISA with commercially available antibodies (i.e., A0082 and P0226; Agilent DAKO, Santa Clara, CA, US).

\section{Glomerular and proximal tubular function}

Serum cystatin C (Particle-enhanced nephelometric immunoassay, Behring Nephelometer II, Siemens Healthineers AG, Munich, Germany) and creatinine (Enzymatic colorimetric assay, Cobas 8000 instrument, Roche Diagnostics, Mannheim, Germany) were determined for the calculation of the eGFR, using the Chronic Kidney Disease Epidemiology Collaboration (CKD-EPI) equation ${ }^{25}$, based on serum creatinine (eGFR $\mathrm{R}_{\text {cr }}$ ), cystatin $\mathrm{C}$ (eGFR $\mathrm{R}_{\mathrm{cys}}$ ) and creatinine and cystatin $C$ combined (eGFR $R_{\text {cr-cys }}$ ). The latter was used as a primary outcome measurement. Proteinuria was determined by measuring the total protein concentration in 24-hour urine samples collected in pre-acidified plastic containers (Turbidimetric assay, Cobas 8000 instrument, Roche Diagnostics, Mannheim, Germany). 
Proximal tubular dysfunction was determined by measuring the concentration of phosphate (Colorimetric assay, Cobas 8000 instrument, Roche Diagnostics, Mannheim, Germany), glucose (D-Glucose/D-Fructose UV-method, RBiopharm AG [produced by Roche Diagnostics, Mannheim, Germany]) and amino acid (ultra-performance liquid chromatography tandem mass spectrometry, Waters, Milford, MA, US ${ }^{26}$ ) in the same 24-hour urine samples. To control for influential variables (e.g., diet and hormones), the tubular reabsorption of phosphate (TRP) and subsequently ratio of tubular maximum reabsorption to GFR (TmP/GFR) was calculated. ${ }^{27}$

\section{Measurements of covariates}

Dietary intake was evaluated using a three-day food journal along with a personal interview by the clinical researcher (N.S.). The weight of each consumed product was either provided by the participant or estimated using average quantities per portion. Macro- and micronutrient composition was determined based on the Dutch food composition table. ${ }^{28}$ Fructose intake was calculated with an extensive sugar composition table, as described previously. ${ }^{11,29}$

Height was determined standing upright against a stadiometer and weight was assessed in solely underwear using an electronic scale. BMI was calculated as weight $(\mathrm{kg})$ divided by height $(\mathrm{m})$ squared. Waist was measured with a measuring tape at the level of the umbilicus.

Blood was drawn for measurement of whole blood glucose (YSI, Yellow Springs, OH, USA), plasma insulin (Human insulin kit, Meso Scale Discovery, Rockville, MD, USA) and serum lipids (Enzymatic colorimetric assay, Cobas 8000 instrument, Roche Diagnostics, Mannheim, Germany).

Urinary sodium concentration (Indirect ISE-method, Cobas 8000 instrument, Roche Diagnostics, Mannheim, Germany) was determined in the 24-hour urine samples.

\section{Statistical analyses}

Data are expressed as median (interquartile range) and analyzed with a MannWhitney $U$ test. Blood pressure, cf-PWV, endothelial function (reactive hyperemia index, heat-induced skin hyperemia, plasma sE-selectin and vWF concentration), glomerular function (eGFR $\mathrm{errcys}_{\text {s }}$ and urinary total protein concentration) and proximal tubular function (TmP/GFR, urinary glucose and 
amino acid concentrations) were considered as the primary outcome measurements. The $95 \%$ confidence interval $(95 \% \mathrm{Cl})$ for the difference $(\Delta)$ between medians of HFI patients versus healthy controls as part of the MannWhitney test was calculated according to the Hodges-Lehmann method. Linear regression analyses were conducted to explore the contribution of potential mediators of any difference in the primary outcome measures. A $\geq 25 \%$ reduction in the unstandardized beta of the primary outcome measurement after inclusion of the potential mediator was considered relevant. Due to the low number of study participants $(n=30)$, linear regression was conducted with only one potential mediator per analysis. Results were considered statistically significant at $p<0.05$. All analyses were carried out with the IBM Statistical Package of Social Sciences (SPSS) version 23 for Windows (SPSS inc. Chicago, IL).

\section{Results}

\section{General characteristics}

Fifteen HFI patients and fifteen age-, sex- and BMI-matched healthy controls were included in this study. General characteristics are displayed in Table 8.1, as published previously. ${ }^{11} \mathrm{HFI}$ patients were relatively young (median age: 31 years), lean (median BMl: $20.4 \mathrm{~kg} / \mathrm{m}^{2}$ ) and predominantly male $(73.3 \%)$. Three out of $15 \mathrm{HFI}$ patients were smokers, as opposed to none of the healthy controls. Alcohol intake was higher among healthy controls (median units per day: 0.6 versus $0.1, p=0.04$ ). As expected, patients consumed almost no fructose (median intake: 1.0 grams/day) and, as a consequence, protein and saturated fat intake were higher compared to healthy controls (median intake: $113.1 \mathrm{grams} /$ day versus $75.4 \mathrm{grams} /$ day, $\mathrm{p}=0.005$ and $37.2 \mathrm{grams} /$ day versus 30.0 grams/day, $p=0.03$, respectively). Serum lipids, whole blood glucose and plasma insulin levels did not differ significantly between both groups. None of the participants used blood pressure-, lipid- or glucose-lowering medication. 
Table 8.1 General characteristics.

\begin{tabular}{|c|c|c|}
\hline & Healthy controls & HFI patients \\
\hline $\operatorname{Sex}(M / F)$ & $11 / 4$ & $11 / 4$ \\
\hline Age $(y)$ & $28(25-52)$ & $31(24-44)$ \\
\hline BMI $\left(\mathrm{kg} / \mathrm{m}^{2}\right)$ & $21.8(21.0-23.3)$ & $20.4(19.3-24.8)$ \\
\hline Waist circumference $(\mathrm{cm})$ & $87(84.3-91.3)$ & $77.5(73.8-93.5)$ \\
\hline IHL content (\%) & $0.59(0.29-1.65)$ & $2.47(0.76-4.56)^{*}$ \\
\hline Liver stiffness (kPa) & $4.3(3.6-5.5)$ & $4.7(4.1-6.2)$ \\
\hline Whole blood glucose $(\mathrm{mmol} / \mathrm{L})$ & $4.4(4.2-4.7)$ & $4.4(4.3-4.8)$ \\
\hline Insulin (pg/ml) & $105(77-159)$ & $115(93-175)$ \\
\hline Total cholesterol (mmol/L) & $4.1(3.9-5.2)$ & $5.0(4.3-6.2)$ \\
\hline HDL cholesterol (mmol/L) & $1.4(1.1-1.8)$ & $1.6(1.4-2.3)$ \\
\hline LDL cholesterol (mmol/L) & $2.4(1.8-3.3)$ & $3.1(2.0-4.2)$ \\
\hline Triglycerides (mmol/L) & $0.9(0.5-1.2)$ & $0.8(0.7-1.3)$ \\
\hline Uric acid $(\mu \mathrm{mol} / \mathrm{L})$ & $334(285-378)$ & 302 (229-339) \\
\hline Caloric intake (kcal/day) & $2058(1552-2316)$ & $2050(1635-2546)$ \\
\hline Protein intake (g/day) & $75.4(70.8-103.9)$ & $113.1(91.1-141.9)^{*}$ \\
\hline Total fat intake (g/day) & $72.3(66.9-83.1)$ & $83.8(70.1-131.5)$ \\
\hline Saturated fat intake (g/day) & $30.0(20.2-33.3)$ & $37.2(29.1-47.2)^{*}$ \\
\hline Carbohydrate intake (g/day) & $237.9(156.0-287.9)$ & $166.3(142.2-215.4)$ \\
\hline Dietary fructose intake (g/day) & $30.6(23.1-48.9)$ & $1.0(0.8-1.5)^{\star}$ \\
\hline Alcohol intake (U/day) & $0.6(0.1-1.7)$ & $0.1(0.0-0.3)^{\star}$ \\
\hline Smoking (\% yes) & $0 \%$ & $20 \%$ \\
\hline
\end{tabular}

Data are expressed as median (interquartile range). ${ }^{*} p<0.05$ versus healthy controls. Analyzed with a MannWhitney $\mathrm{U}$ test. Parts of this table have already been published. ${ }^{11}$

\section{Blood pressure and pulse wave velocity}

Both systolic and diastolic blood pressure were higher in HFI patients, of which only the former reached statistical significance $(p=0.045, \Delta: 10 \mathrm{mmHg}, 95 \% \mathrm{Cl}$ : $1 ; 20$ and $p=0.056, \Delta: 8 \mathrm{mmHg}, 95 \% \mathrm{Cl}:-1 ; 16$; Figure 8.1 , panel $\mathrm{A}$ and $\mathrm{B}$, respectively). Five HFI patients had a systolic blood pressure $\geq 140 \mathrm{mmHg}$ and/or diastolic blood pressure $\geq 90 \mathrm{mmHg}$, as opposed to one healthy control individual. Heart rate was not statistically different between HFI patients and healthy controls $(p=0.54, \Delta: 2 \mathrm{bpm}, 95 \% \mathrm{Cl}:-5 ; 12)$. Pulse pressure and cf-PWV were not different either $(p=0.37, \Delta: 2 \mathrm{mmHg}, 95 \% \mathrm{Cl}:-4 ; 11$ and $p=0.49$, $\Delta: 0.5 \mathrm{~m} / \mathrm{s}, 95 \% \mathrm{Cl}:-1.4 ; 2.9$; Figure 8.1 , panel $\mathrm{C}$ and $\mathrm{D}$, respectively). Dietary intake of sodium was not different between HFI patients and healthy controls $(p=0.56, \Delta: 437 \mathrm{mg}, 95 \% \mathrm{Cl}$ : $-270 ; 880$; Figure 8.1 , panel $\mathrm{E})$. In contrast, urinary sodium excretion was higher in HFI patients $(p=0.01, \Delta: 47.1 \mathrm{mmol} / \mathrm{day}$, 95\% Cl: 9.1;81.1; Figure 8.1, panel F). Linear regression analyses were conducted to explore the role of smoking, cf-PWV, dietary sodium intake or urinary sodium excretion in the observed difference in systolic blood pressure. None of these had a substantial effect on the observed difference between HFI 
patients and controls (reduction in unstandardized beta of the affected state [HFI yes/no] after addition of the potential mediator: $<25 \%$, data not shown).
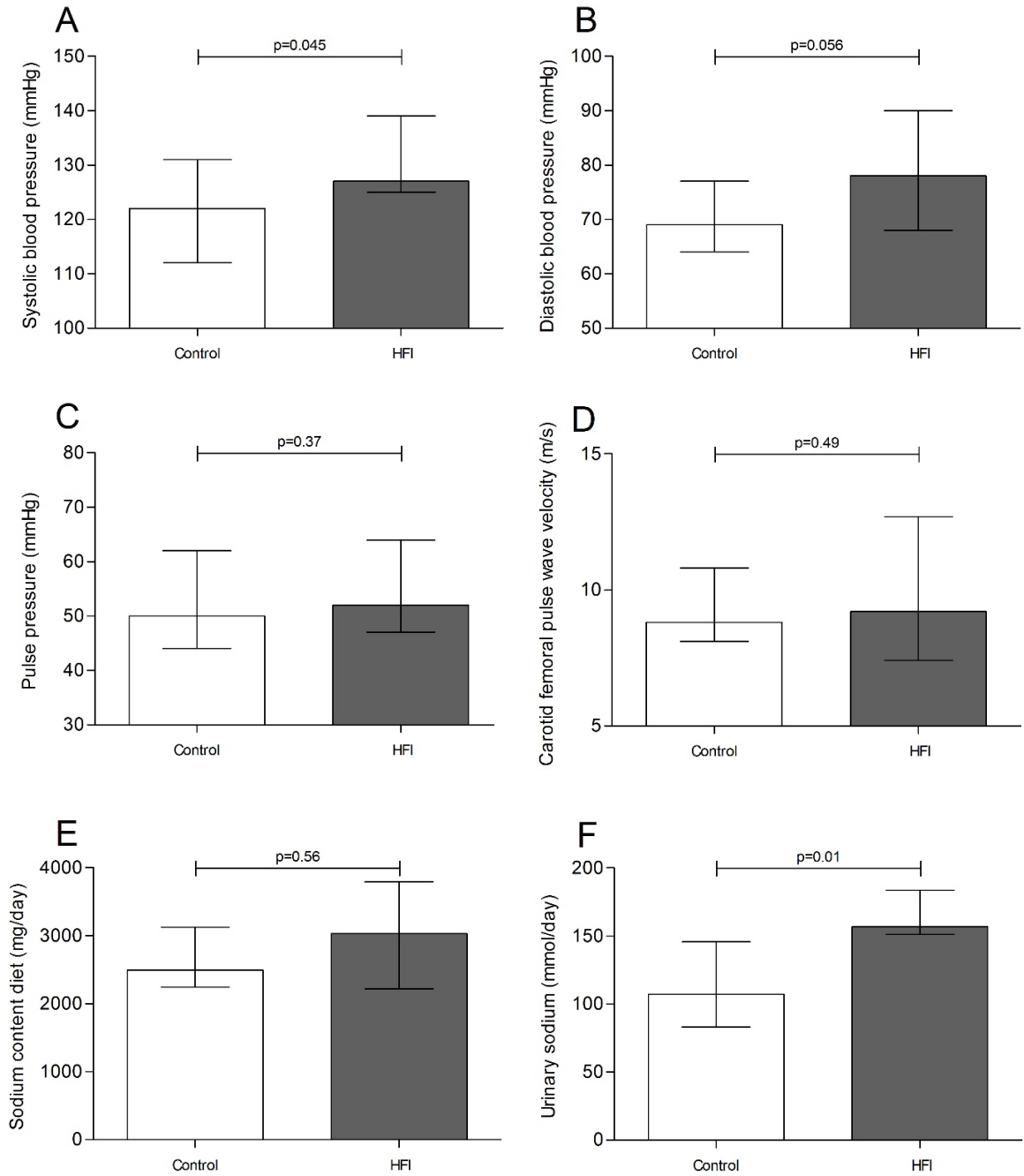

Figure 8.1 Blood pressure, aortic stiffness and sodium intake and excretion. Systolic blood pressure (panel A), diastolic blood pressure (panel B), pulse pressure (panel C), carotid-femoral pulse wave velocity (panel D), daily sodium intake (panel E) and 24-hour urinary sodium excretion (panel $F$ ) in HFI patients (grey bars) and healthy controls (white bars). Data are presented as medians (interquartile range). Analyzed with a Mann-Whitney $U$ test. 


\section{Endothelial function}

The reactive hyperemia index and the percentage heat-induced skin hyperemia were not significantly different between $\mathrm{HFI}$ patients and healthy controls $(p=0.75, \Delta: 0.09,95 \% \mathrm{Cl}:-0.31 ; 0.53$ and $\mathrm{p}=0.43, \Delta: 316,95 \% \mathrm{Cl}:-349 ; 992$; Figure 8.2, panel $\mathrm{A}$ and $\mathrm{B}$, respectively). Plasma $\mathrm{sE}$-selectin was significantly higher in HFI patients as compared to healthy controls $(p=0.004, \Delta: 46 \mathrm{ng} / \mathrm{ml}$, $95 \% \mathrm{Cl}: 19 ; 71$; Figure 8.2, panel $\mathrm{C}$ ), whereas no significant difference was found for plasma vWF ( $p=0.95, \Delta: 1.5 \%, 95 \% \mathrm{Cl}$ : $-19 ; 20$; Figure 8.2 , panel $\mathrm{D})$. Linear regression analyses were conducted to explore the role of plasma glucose, serum lipids, intrahepatic lipid content, alcohol intake, blood pressure, smoking behavior and eGFR $\mathrm{cr}_{\text {crcys }}$ in the observed difference in plasma sEselectin. None of these caused a substantial reduction in the observed difference between $\mathrm{HFI}$ patients and controls.
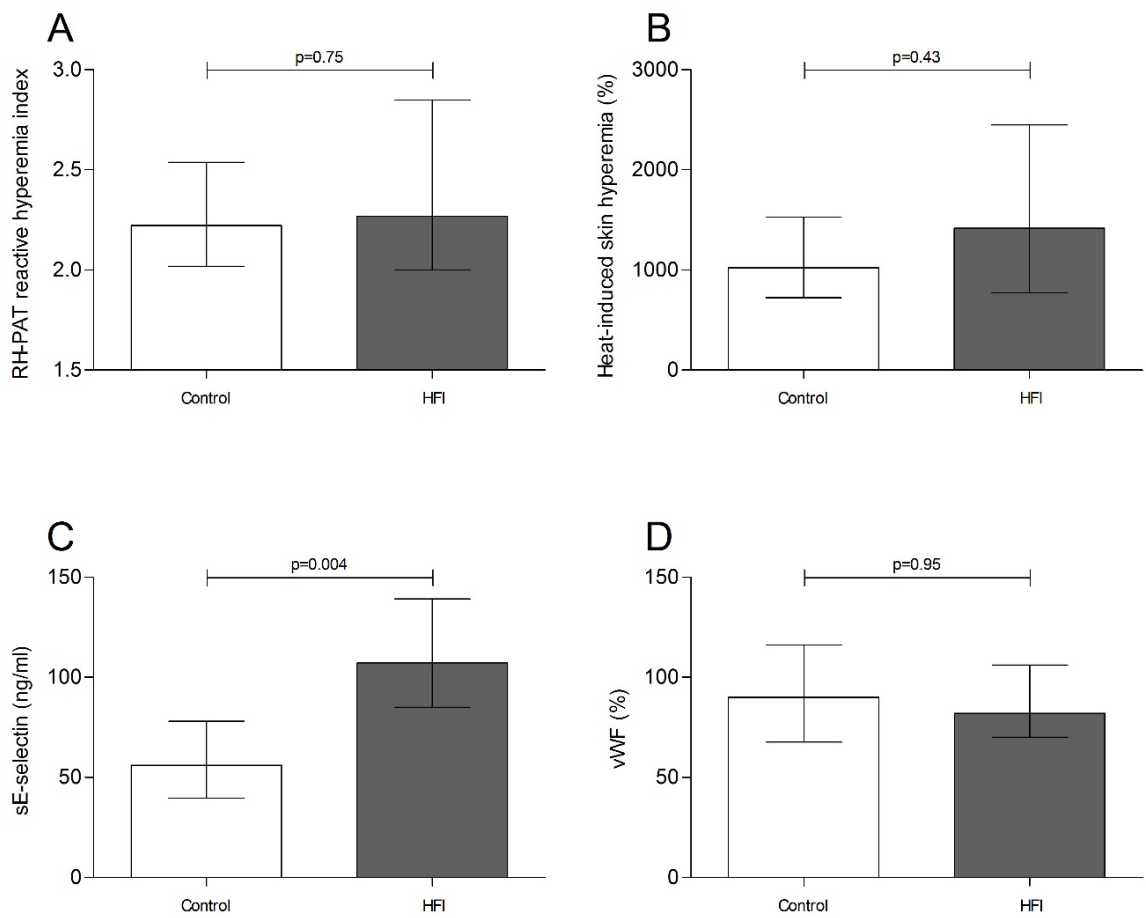

Figure 8.2 Endothelial function. Ischemia induced reactive hyperemia (panel A), heat-induced skin hyperemia (panel B), plasma sE-selectin levels (panel C) and plasma von Willebrand factor levels (panel D) in HFI patients (grey bars) and healthy controls (white bars). Data are presented as medians (interquartile range). Analyzed with a Mann-Whitney $U$ test. 


\section{Glomerular and proximal tubular function}

The eGFR $R_{c r}$ and $e G F R_{\text {cr-cys }}$ were significantly higher in $\mathrm{HFI}$ patients as compared to healthy controls $\left(p<0.001, \Delta: 19 \mathrm{ml} / \mathrm{min} / 1.73 \mathrm{~m}^{2}, 95 \% \mathrm{Cl}: 10 ; 29\right.$ and $p=0.001, \Delta: 16 \mathrm{ml} / \mathrm{min} / 1.73 \mathrm{~m}^{2}, 95 \% \mathrm{Cl}: 6 ; 24$, respectively; Figure 8.3 , panel A). Although the estimate of difference for eGFR cys $_{\text {chas }}$ in a similar range $(\Delta 11$ $\left.\mathrm{ml} / \mathrm{min} / 1.73 \mathrm{~m}^{2}, 95 \% \mathrm{Cl}:-2 ; 20\right)$, it did not reach statistical significance $(p=0.10)$. None of the participants had an eGFR $<60 \mathrm{ml} / \mathrm{min} / 1.73 \mathrm{~m}^{2}$ (compatible with chronic kidney disease stage $3 \mathrm{a}$ ). Linear regression analyses were conducted to explore the role of systolic blood pressure and dietary protein intake, which are known risk factors for glomerular hyperfiltration ${ }^{30,31}$, in the observed difference in eGFR $\mathrm{crccys}_{\text {. }}$. The unstandardized beta of the HFI affected state did not substantially decline after addition of these variables to the model (data not shown). Urinary total protein concentration was not statistically different between both groups $(p=0.26, \Delta: 0.0$ grams/day, $95 \% \mathrm{Cl}:-0.03 ; 0.0$; Figure 8.3, panel B).

TmP/GFR and urinary glucose were not statistically different between both groups, as shown in Figure 8.3, panel $C$ and $D(p=0.94, \Delta: 0.005,95 \% \mathrm{Cl}$ : $-0.22 ; 0.18$ and $\mathrm{p}=0.55, \Delta: 5.9 \mathrm{mg} /$ day, $95 \% \mathrm{Cl}:-12.4 ; 21.8$, respectively). No statistical differences in urinary amino acid concentrations were found between $\mathrm{HFI}$ patients and healthy controls (Figure 8.3, panel E). Alanine (Ala), valine (Val), leucine (Leu) and phenylalanine (Phe) concentrations tended to be higher in HFI patients $(p=0.06, \Delta: 7.7 \mu \mathrm{MmM}$ creatinine, $95 \% \mathrm{Cl}:-1.1 ; 17.4$, $\mathrm{p}=0.08, \Delta: 1.2 \mu \mathrm{MmM}$ creatinine, $95 \% \mathrm{Cl}:-0.2 ; 2.5, \mathrm{p}=0.10, \Delta: 0.6 \mu \mathrm{MmM}$ creatinine, $95 \% \mathrm{Cl}:-0.07 ; 1.2$ and $\mathrm{p}=0.08, \Delta: 1.4 \mu \mathrm{MmM}$ creatinine, $95 \% \mathrm{Cl}$ : $-0.06 ; 2.7$, respectively). 
A

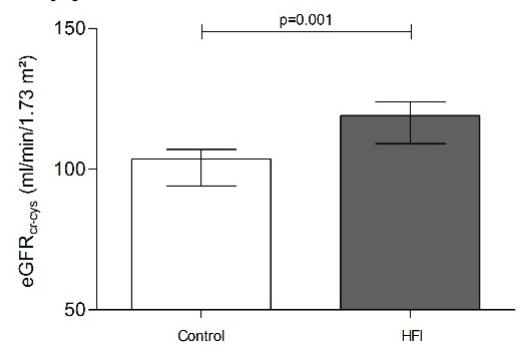

\section{C}

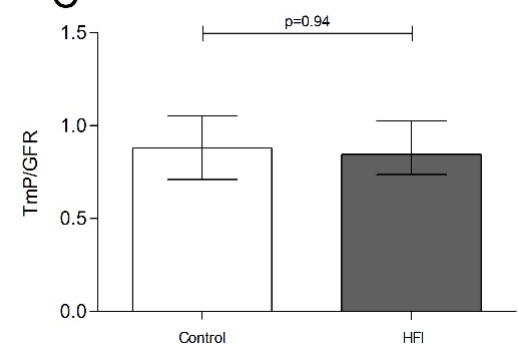

B
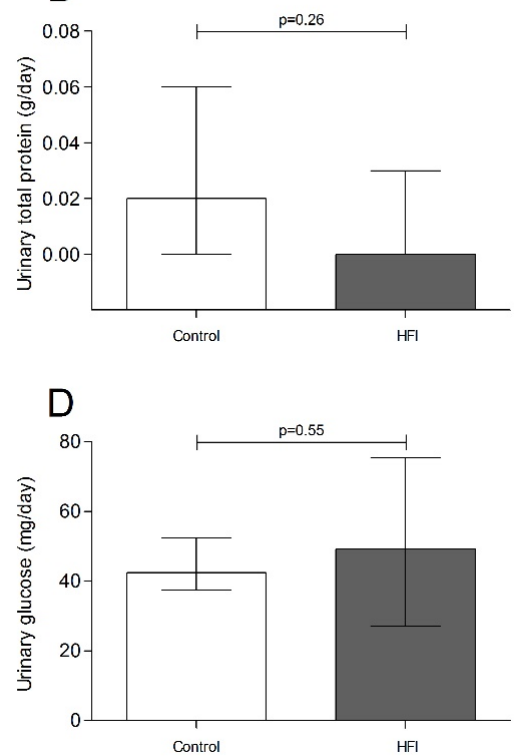

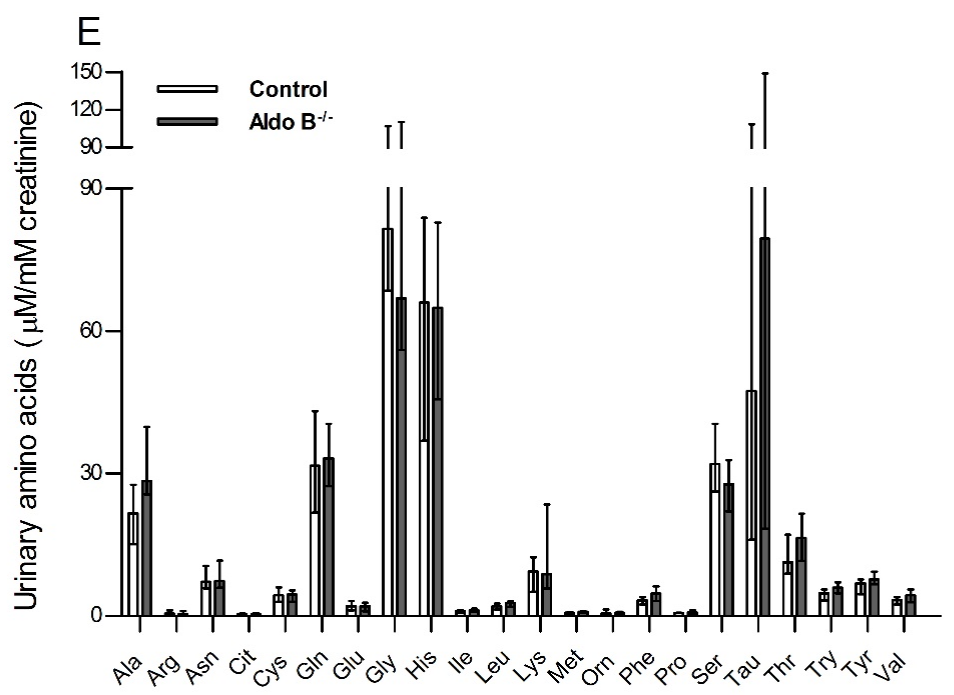

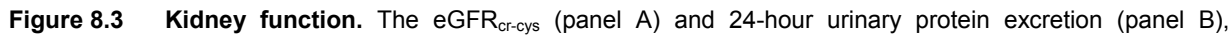
TmP/GFR (panel C), 24-hour urinary glucose excretion (panel D) and 24-hour urinary amino acid excretion (panel E) in HFI patients (grey bars) and healthy controls (white bars). Data are presented as medians (interquartile range). Analyzed with a Mann-Whitney $U$ test. Abbreviations: Alanine (Ala), Arginine (Arg), Asparagine (Asn), Citrulline (Cit), Cysteine (Cys), Glutamine (Gln), Glutamic acid (Glu), Glycine (Gly), Histidine (His), Isoleucine (lle), Leucine (Leu), Lysine (Lys), Methionine (Met), Ornithine (Orn), Phenylalanine (Phe), Proline (Pro), Serine (Ser), Taurine (Tau), Threonine (Thr), Tryptophan (Try), Tyrosine (Tyr), Valine (Val). 


\section{Discussion}

$\mathrm{HFI}$ is an inborn error of metabolism that can be treated with a fructoserestricted diet. Up to now, mostly case reports on $\mathrm{HFI}$ at the time of diagnosis have been published. ${ }^{32-34}$ We and others previously showed that HFI patients treated with a fructose-restricted diet are characterized by a greater intrahepatic lipid content. $^{11-13}$ In the present study, we investigated the longterm consequences of $\mathrm{HFI}$ on other aldolase-B-expressing organs and found that HFI patients have a higher systolic blood pressure, eGFR and sE-selectin levels compared to healthy controls matched for age, sex and BMI.

HFI patients displayed a higher systolic blood pressure than healthy controls. Diastolic blood pressure tended to be higher as well $(p=0.056)$. Five of 15 patients had systolic blood pressure $\geq 140 \mathrm{mmHg}$ and/or diastolic blood pressure $\geq 90 \mathrm{mmHg}$. Since blood pressure was determined only twice during the study visit, we cannot exclude white coat hypertension as a potential explanation for the observed difference. Ideally, this would require 24-hour blood pressure monitoring. ${ }^{35}$ To explore the contribution of salt intake, which affects both systolic and diastolic blood pressure as an alternative explanation $^{36}$, we determined dietary sodium intake and 24-hour urinary sodium excretion. Although the former was not statistically different between both groups, we did find a significantly higher 24-hour urinary sodium excretion in HFI patients. Differences between intake and urinary excretion of sodium have been reported in other studies as well ${ }^{37,38}$ and is most likely the result of inaccurate food recording. The National Academy of Medicine (formerly known as the Institute of Medicine) considers analysis of 24-hour urine collection a reliable method of sodium intake estimation. ${ }^{39}$ Regression analyses, however, did not show a substantial reduction in the unstandardized beta after addition of these variables to the model. Although these analyses suggest that dietary salt intake is not a major explanatory variable for the observed difference in systolic blood pressure, these exploratory analyses should be interpreted with caution given the relatively small study population under investigation. Similar conclusions can be drawn for the potential mediating role of aortic stiffness (approximated by cf-PWV) in explaining the higher systolic blood pressure in $\mathrm{HFI}$. Regardless of the exact cause, it is clinically advisable to perform routine blood pressure measurements in HFI patients during outpatient visits.

Previous studies have shown that ingestion of fructose by HFI patients leads to proximal tubular dysfunction (i.e., Fanconi syndrome) and eventually to renal failure. $^{9,40}$ In the present study, proximal tubular function, reflected by TmP/GFR, urinary glucose and amino acid excretion, did not differ significantly 
between adult HFI patients treated with a fructose-restricted diet and healthy controls. Although the urinary alanine, valine, leucine and phenylalanine concentrations tended to be higher in HFI patients, all were within the normal range in comparison to the amino acid excretion as seen in patients with Fanconi syndrome. ${ }^{41}$ In contrast, the $\mathrm{eGFR}_{\mathrm{cr}-\mathrm{cys}}$ was significantly higher in $\mathrm{HFI}$ patients than healthy controls. It is unlikely that this difference is explained by a difference in body (muscle) mass or diet - which affect serum creatinine levels $^{42,43}$ - since a similar effect size was observed for eGFR cys $_{\text {(albeit not }}$ statistically significant, $p=0.10)$.

A higher eGFR could reflect glomerular hyperfiltration, as seen in other disease states such as diabetes mellitus ${ }^{44}$, which could be a precursor of chronic kidney disease. $^{45}$ In the general population, a high GFR has been associated with an increase in albumin-to-creatinine ratio and incident albuminuria. ${ }^{46}$ Of interest, both dietary protein intake and systolic blood pressure (which were found to be higher in $\mathrm{HFI}$ patients) are associated with glomerular hyperfiltration. ${ }^{30,31}$ In addition, we previously showed that HFI patients are more glucose intolerant ${ }^{11}$, which might contribute to glomerular hyperfiltration as well. ${ }^{47}$ Regression analyses with systolic blood pressure and dietary protein intake as potential mediators did, however, not reveal a substantial reduction in the unstandardized beta. Again, a mediating role of these factors in explaining the higher eGFR in HFI patients cannot fully be excluded.

On the other hand, a higher eGFR in HFI might also reflect a truly better glomerular function. Although speculative, a hepatorenal axis in HFI patients may account for the current observations. We and others previously showed that HFI patients are also characterized by greater intrahepatic lipid content than controls. ${ }^{11-13}$ Detailed studies in aldolase B deficient mice suggest that an increased dissociation of hepatic glucokinase from glucokinase regulatory protein may explain the greater intrahepatic lipid content in $\mathrm{HFI}^{48}$ Of interest, variants in the GCKR gene (that encode a glucokinase regulatory protein that binds glucokinase less effectively ${ }^{49}$ ) have been associated with both an increased intrahepatic lipid content and a higher eGFR in the general population. ${ }^{50,51}$ The same genetic variants tended to protect from chronic kidney disease as well $(p=0.13){ }^{51}$ Moreover, liver-specific knockout of glucokinase results in increased kidney damage. ${ }^{52}$ These suggestions of a hepatorenal axis in HFI deserve further study.

Of all measures of endothelial function, only plasma sE-selectin levels, an endothelium-specific biomarker, were significantly higher in $\mathrm{HFI}$ patients compared to healthy controls. The fact that plasma vWF, LDF and RH-PAT 
were not statistically different between HFI patients and healthy controls, might be due to a lack of statistical power, or alternatively, a difference in the type of blood vessel under investigation, i.e., small arteries (RH-PAT) versus skin arterioles (LDF) versus the microcirculation in general (plasma sE-selectin and vWF). Of note, previous epidemiological studies have shown that $s E-$ selectin levels predict future type 2 diabetes and cardiovascular disease. ${ }^{53,54} \mathrm{We}$ previously showed that adult HFI patients treated with a fructose-restricted diet are more glucose intolerant than healthy controls. ${ }^{11}$

This study has strengths and limitations. As hitherto mentioned, the small patient population makes it difficult to draw conclusions on non-significant results. Therefore, these results should be interpreted with caution. In addition, formal mediation analyses are required to explore the contribution of potential mediators to the difference in primary outcomes measures between HFI patients and healthy controls. Due to the low number of study participants, however, this was not possible. Last, the healthy control subjects, who were matched for age, sex and BMI, do not necessarily represent the general population. This is the first report of HFI patients treated with a fructoserestricted diet using state of the art methods. Since only case reports on HFI at time of diagnosis have been published ${ }^{32-34}$, this study provides useful information for the long-term management of HFI patients.

In conclusion, following our previous report on the presence of a higher intrahepatic lipid content, liver fibrosis and glucose intolerance ${ }^{11}$, we now show that the adult HFI patients treated with a fructose-restricted diet are characterized by a higher systolic blood pressure, eGFR and soluble E-selectin level compared to age-, sex- and BMI-matched healthy controls. Further studies are required to elucidate the exact causes of these observations, either the direct consequence of the genetic defect or secondary to its dietary treatment. These results do emphasize the need for long-term follow-up of HFI patients, in particular with regard to blood pressure and kidney function. 


\section{Literature}

1. Ali M, Rellos P, Cox TM. Hereditary fructose intolerance. J Med Genet 1998;35:353-65.

2. Cross NC, de Franchis R, Sebastio G, et al. Molecular analysis of aldolase $B$ genes in hereditary fructose intolerance. Lancet 1990;335:306-9.

3. Fagerberg L, Hallstrom BM, Oksvold $P$, et al. Analysis of the human tissue-specific expression by genome-wide integration of transcriptomics and antibody-based proteomics. Mol Cell Proteomics : MCP 2014;13:397-406.

4. Zubay G. Biochemistry. Reading, MA: Addison-Wesley; 1986.

5. Odievre M, Gentil C, Gautier M, Alagille D. Hereditary fructose intolerance in childhood. Diagnosis, management, and course in 55 patients. Am J Dis Child 1978;132:605-8.

6. Baerlocher K, Gitzelmann R, Steinmann B, Gitzelmann-Cumarasamy N. Hereditary fructose intolerance in early childhood: a major diagnostic challenge. Survey of 20 symptomatic cases. Helv Paediatr Acta 1978;33:465-87.

7. Mock DM, Perman JA, Thaler M, Morris RC, Jr. Chronic fructose intoxication after infancy in children with hereditary fructose intolerance. A cause of growth retardation. $\mathrm{N}$ Engl J Med 1983;309:764-70.

8. von Ruecker A, Endres W, Shin YS, Butenandt I, Steinmann B, Gitzelmann R. A case of fatal hereditary fructose intolerance. Misleading information of formula composition. Helv Paediatr Acta 1981;36:599-600.

9. Levin B, Snodgrass GJ, Oberholzer VG, Burgess EA, Dobbs RH. Fructosaemia. Observations on seven cases. Am J Med 1968;45:826-38.

10. Fernandes J, Saudubray J, vd Berghe G, Walter J. Inborn Metabolic Diseases. Heidelberg, Germany: Springer Medizin Verlag; 2006.

11. Simons N, Debray FG, Schaper NC, et al. Patients With Aldolase B Deficiency Are Characterized by Increased Intrahepatic Triglyceride Content. J Clin Endocrinol Metab 2019;104:5056-64.

12. Aldamiz-Echevarria L, de Las Heras J, Couce ML, et al. Non-alcoholic fatty liver in hereditary fructose intolerance. Clin Nutr 2020;39:455-9.

13. Di Dato F, Spadarella S, Puoti MG, et al. Daily Fructose Traces Intake and Liver Injury in Children with Hereditary Fructose Intolerance. Nutrients 2019;11.

14. Liu J, Mak TC, Banigesh A, Desai K, Wang R, Wu L. Aldolase B knockdown prevents high glucose-induced methylglyoxal overproduction and cellular dysfunction in endothelial cells. PloS One 2012;7:e41495.

15. Cao W, Chang T, Li XQ, Wang R, Wu L. Dual effects of fructose on ChREBP and FoxO1/3alpha are responsible for AldoB up-regulation and vascular remodelling. Clin Sci (Lond) 2017;131:309-25.

16. Liu J, Wang R, Desai K, Wu L. Upregulation of aldolase B and overproduction of methylglyoxal in vascular tissues from rats with metabolic syndrome. Cardiovasc Res 2011;92:494-503.

17. World Medical Association Declaration of Helsinki: ethical principles for medical research involving human subjects. JAMA 2013;310:2191-4.

18. Stehouwer CD, Henry RM, Ferreira I. Arterial stiffness in diabetes and the metabolic syndrome: a pathway to cardiovascular disease. Diabetologia 2008;51:527-39.

19. Laurent $\mathrm{S}$, Cockcroft J, Van Bortel L, et al. Expert consensus document on arterial stiffness: methodological issues and clinical applications. Eur Heart J 2006;27:2588-605.

20. Stehouwer CDA. Microvascular Dysfunction and Hyperglycemia: A Vicious Cycle With Widespread Consequences. Diabetes 2018;67:1729-41.

21. Bonetti PO, Pumper GM, Higano ST, Holmes DR, Jr., Kuvin JT, Lerman A. Noninvasive identification of patients with early coronary atherosclerosis by assessment of digital reactive hyperemia. J Am Coll Cardiol 2004;44:2137-41. 
22. Rajan V, Varghese B, van Leeuwen TG, Steenbergen W. Review of methodological developments in laser Doppler flowmetry. Lasers Med Sci 2009;24:269-83.

23. Sorensen BM, Houben AJ, Berendschot TT, et al. Prediabetes and Type 2 Diabetes Are Associated With Generalized Microvascular Dysfunction: The Maastricht Study. Circulation 2016;134:1339-52.

24. Choi PJ, Brunt VE, Fujii N, Minson CT. New approach to measure cutaneous microvascular function: an improved test of NO-mediated vasodilation by thermal hyperemia. J Appl Physiol (1985) 2014;117:277-83.

25. Levey AS, Stevens $\mathrm{LA}$, Schmid $\mathrm{CH}$, et al. A new equation to estimate glomerular filtration rate. Ann Intern Med 2009;150:604-12.

26. Waterval WA, Scheijen JL, Ortmans-Ploemen MM, Habets-van der Poel CD, Bierau J. Quantitative UPLC-MS/MS analysis of underivatised amino acids in body fluids is a reliable tool for the diagnosis and follow-up of patients with inborn errors of metabolism. Clin Chim Acta 2009;407:36-42.

27. Payne RB. Renal tubular reabsorption of phosphate (TmP/GFR): indications and interpretation. Ann Clin Biochem 1998;35 ( Pt 2):201-6.

28. Rijksinstituut voor Volksgezondheid en Milieu (National Institute for Public Health and the Environment) (2010) NEVO-tabel (Dutch Food Composition Table). Nederlands Voedingsstoffenbestand 2010 (Dutch Food Composition Database 2010). Den Haag: RIVM.

29. Sluik D, Engelen Al, Feskens EJ. Fructose consumption in the Netherlands: the Dutch National Food Consumption Survey 2007-2010. Eur J Clin Nutr 2015;69:475-81.

30. Okada R, Yasuda Y, Tsushita K, Wakai K, Hamajima N, Matsuo S. Glomerular hyperfiltration in prediabetes and prehypertension. Nephrol Dial Transplant 2012;27:1821-5.

31. Schwingshackl L, Hoffmann G. Comparison of high vs. normal/low protein diets on renal function in subjects without chronic kidney disease: a systematic review and meta-analysis. PloS One 2014;9:e97656.

32. Chambers RA, Pratt RT. Idiosyncrasy to fructose. Lancet (London, England) 1956;271:340.

33. Froesch ER, Wolf HP, Baitsch H, Prader A, Labhart A. Hereditary fructose intolerance. An inborn defect of hepatic fructose-1-phosphate splitting aldolase. Am J Med 1963;34:151-67.

34. Esposito G, Imperato MR, leno L, et al. Hereditary fructose intolerance: functional study of two novel ALDOB natural variants and characterization of a partial gene deletion. Hum Mutat 2010;31:1294-303.

35. Whelton PK, Carey RM, Aronow WS, et al. 2017 ACC/AHA/AAPA/ABC/ACPM/AGS/APhA/ ASH/ASPC/NMA/PCNA Guideline for the Prevention, Detection, Evaluation, and Management of High Blood Pressure in Adults: A Report of the American College of Cardiology/American Heart Association Task Force on Clinical Practice Guidelines. Hypertension (Dallas, Tex : 1979) 2018;71:e13-e115.

36. Sacks FM, Svetkey LP, Vollmer WM, et al. Effects on blood pressure of reduced dietary sodium and the Dietary Approaches to Stop Hypertension (DASH) diet. DASH-Sodium Collaborative Research Group. N Engl J Med 2001;344:3-10.

37. Kirkendall AM, Connor WE, Abboud F, Rastogi SP, Anderson TA, Fry M. The effect of dietary sodium chloride on blood pressure, body fluids, electrolytes, renal function, and serum lipids of normotensive man. J Lab Clin Med 1976;87:411-34.

38. Schachter J, Harper PH, Radin ME, Caggiula AW, McDonald RH, Diven WF. Comparison of sodium and potassium intake with excretion. Hypertension 1980;2:695-9.

39. Institute of Medicine. Strategies to Reduce Sodium Intake in the United States. Washington, DC: National Academies Press; 2010.

40. Lameire N, Mussche M, Baele G, Kint J, Ringoir S. Hereditary fructose intolerance: a difficult diagnosis in the adult. Am J Med 1978;65:416-23.

41. Norden AG, Sharratt P, Cutillas PR, Cramer R, Gardner SC, Unwin RJ. Quantitative amino acid and proteomic analysis: very low excretion of polypeptides $>750 \mathrm{Da}$ in normal urine. Kidney Int 2004;66:1994-2003. 
42. Vinge E, Lindergard B, Nilsson-Ehle P, Grubb A. Relationships among serum cystatin C, serum creatinine, lean tissue mass and glomerular filtration rate in healthy adults. Scand $J$ Clin Lab Invest 1999;59:587-92.

43. Tangri N, Stevens $\mathrm{LA}$, Schmid $\mathrm{CH}$, et al. Changes in dietary protein intake has no effect on serum cystatin $C$ levels independent of the glomerular filtration rate. Kidney Int 2011;79: 471-7.

44. Levine DZ. Can rodent models of diabetic kidney disease clarify the significance of early hyperfiltration?: recognizing clinical and experimental uncertainties. Clin Sci (Lond) 2008;114:109-18.

45. Helal I, Fick-Brosnahan GM, Reed-Gitomer B, Schrier RW. Glomerular hyperfiltration: definitions, mechanisms and clinical implications. Nat Rev Nephrol 2012;8:293-300.

46. Melsom T, Stefansson V, Schei J, et al. Association of Increasing GFR with Change in Albuminuria in the General Population. Clin J Am Soc Nephrol 2016;11:2186-94.

47. Sun ZJ, Yang YC, Wu JS, Wang MC, Chang CJ, Lu FH. Increased risk of glomerular hyperfiltration in subjects with impaired glucose tolerance and newly diagnosed diabetes. Nephrol Dial Transplant 2016;31:1295-301.

48. Lanaspa MA, Andres-Hernando A, Orlicky DJ, et al. Ketohexokinase C blockade ameliorates fructose-induced metabolic dysfunction in fructose-sensitive mice. J Clin Invest 2018;128:2226-38.

49. Beer NL, Tribble ND, McCulloch LJ, et al. The P446L variant in GCKR associated with fasting plasma glucose and triglyceride levels exerts its effect through increased glucokinase activity in liver. Hum Mol Genet 2009;18:4081-8.

50. Speliotes EK, Yerges-Armstrong LM, Wu J, et al. Genome-wide association analysis identifies variants associated with nonalcoholic fatty liver disease that have distinct effects on metabolic traits. PLoS Genet 2011;7:e1001324.

51. Simons P, Simons N, Stehouwer CDA, Schalkwijk CG, Schaper NC, Brouwers M. Association of common gene variants in glucokinase regulatory protein with cardiorenal disease: A systematic review and meta-analysis. PloS One 2018;13:e0206174.

52. Gu Y, Mao Y, Li H, et al. Long-term renal changes in the liver-specific glucokinase knockout mouse: implications for renal disease in maturity-onset diabetes of the young 2. Transl Res 2011;157:111-6.

53. Meigs JB, Hu FB, Rifai N, Manson JE. Biomarkers of endothelial dysfunction and risk of type 2 diabetes mellitus. JAMA 2004;291:1978-86.

54. Hwang SJ, Ballantyne CM, Sharrett AR, et al. Circulating adhesion molecules VCAM-1, ICAM-1, and E-selectin in carotid atherosclerosis and incident coronary heart disease cases: the Atherosclerosis Risk In Communities (ARIC) study. Circulation 1997;96:4219-25. 


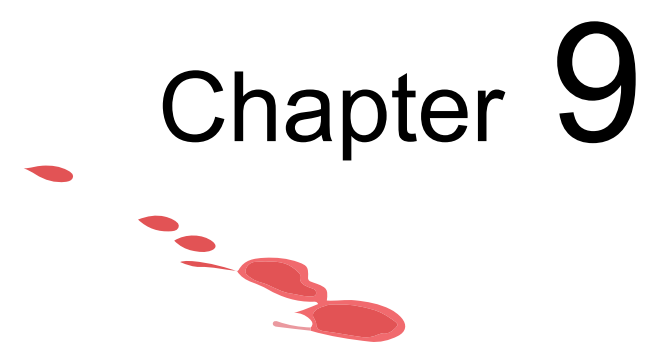

\section{Intrahepatic lipid content after a six-week fructose-restricted diet}
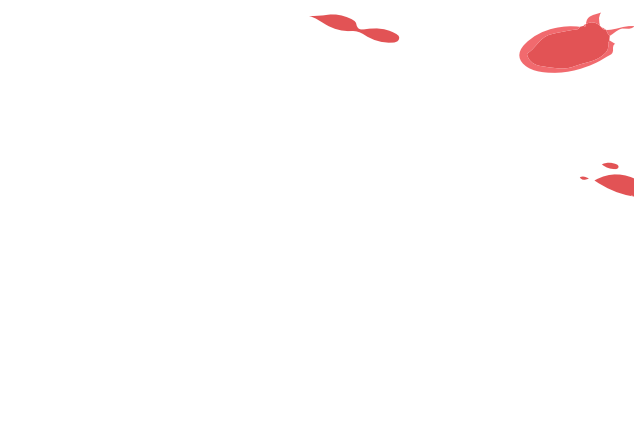

Effects of fructose restriction on liver steatosis (FRUITLESS);

a double-blind randomized controlled trial

Nynke Simons, Pandichelvam Veeraiah, Pomme I.H.G. Simons, Nicolaas C. Schaper, M. Eline Kooi, Vera B. Schrauwen-Hinderling, Edith J.M.Feskens, E.M.C. (Liesbeth) van der Ploeg, Mathias D.G. van den Eynde, Casper G. Schalkwijk, Coen D.A. Stehouwer, Martijn C.G.J. Brouwers 


\section{Abstract}

\section{Background}

There is an ongoing debate on whether fructose plays a role in the development of nonalcoholic fatty liver disease (NAFLD). The aim of this study was to investigate the effects of fructose restriction on intrahepatic lipid (IHL) content in a double-blind randomized controlled trial using an isocaloric comparator.

\section{Methods}

Between March 2017 and October 2019, forty-four adult overweight individuals with a fatty liver index $\geq 60$ were randomly assigned to a six-week fructoserestricted diet (<7.5 grams per meal and <10 grams per day) supplemented with sachets of glucose (=intervention group) or fructose (=control group) three times daily. Participants and assessors were blinded to the allocation. IHL content, assessed by proton magnetic resonance spectroscopy, was the primary outcome and glucose tolerance and serum lipids were the secondary outcomes. All measurements were conducted in Maastricht University Medical Center.

\section{Results}

Thirty-seven participants completed the study protocol. After six weeks of fructose restriction, dietary fructose intake and urinary fructose excretion were significantly lower in the intervention group (difference: -57.0 grams/day, 95\%Cl:-77.9;-39.5 and $-38.8 \mu \mathrm{mol} /$ day, 95\%Cl:-91.2;-10.7, respectively). Although $\mathrm{IHL}$ content decreased in both the intervention and control group $(p<0.001$ and $p=0.003$, respectively), the change in $\mathrm{IHL}$ content was more pronounced in the intervention group (difference: -0.7 percent point, $95 \% \mathrm{Cl}$ :$2.0 ;-0.03)$. The change in glucose tolerance and serum lipids were not different between both groups.

\section{Conclusions}

Six weeks of fructose restriction leads to a small but statistically significant decrease in IHL content. 


\section{Background}

The drastic increase in fructose consumption since the industrial revolution has paralleled the current epidemic of obesity and its cardiometabolic complications, such as dyslipidemia, type 2 diabetes, nonalcoholic fatty liver disease (NAFLD) and cardiovascular disease. ${ }^{1-4}$ Furthermore, fructose overfeeding trials have convincingly shown to increase the accumulation of lipids in the liver ${ }^{5}$, the principal organ involved in the metabolism of fructose. ${ }^{6}$

There is, however, an ongoing debate on whether fructose is more detrimental for liver health than other simple sugars, such as glucose ${ }^{6,7}$ A previous metaanalysis did not show any effects of isocaloric fructose exchange for other carbohydrates on intrahepatic lipid (IHL) content or serum alanine aminotransferase levels. ${ }^{5}$ On the other hand, Schwarz and colleagues more recently demonstrated that a nine-day isocaloric fructose-restricted diet successfully reduced IHL content in obese children with high habitual sugar intake, which was mainly attributed to a decrease in de novo lipogenesis. ${ }^{8}$

The discrepancies between these studies may be attributed to the amount of fructose that was consumed, i.e., either moderate to high $^{5}$ or very low. ${ }^{8}$ Fructose can serve as a substrate for de novo lipogenesis and stimulate de novo lipogenesis via upregulation of transcription factors that enable the expression of genes involved in de novo lipogenesis, such as sterol regulatory element binding protein 1 (SREBP1), carbohydrate response element binding protein (ChREBP) and liver $X$ receptor (LXR) ${ }^{9,10}$ On the other hand, even at small ('catalytic') amounts, fructose can also increase hepatic glucose disposal. ${ }^{11,12}$

As the interpretation of the intervention study by Schwarz and colleagues was limited by its single-arm design and, hence, the lack of a control group, the aim of the current study ('eFfects of fRUctose restrlcTion on LivEr SteatosiS': FRUITLESS) was to investigate the effects of fructose restriction on IHL content in a double-blind randomized controlled trial, using an isocaloric comparator. 


\section{Methods}

\section{Study population}

Between March 2017 and October 2019, individuals were recruited via advertisements or contacted directly if they had participated in scientific research before and agreed to be contacted for future studies. Inclusion criteria for participation were age $\geq 18$ years, a high prior chance of having an increased IHL content (i.e., a body mass index (BMI) $\geq 28 \mathrm{~kg} / \mathrm{m}^{2}$ and a fatty liver index $(\mathrm{FLI}) \geq 60^{13,14}$ ) and a daily fructose intake above the Dutch average (i.e., $\geq 45$ grams $/$ day ${ }^{15}$ according to a three-day food journal). If an individual did not meet the inclusion criterion of $\mathrm{BMI} \geq 28 \mathrm{~kg} / \mathrm{m}^{2}$, but had an elevated $\mathrm{FLI}$, he/she was still considered eligible to participate. Due to a slow recruitment rate, the inclusion criterion of fructose intake $\geq 45$ grams/day was abandoned (protocol amendment July 18, 2018). Individuals were excluded from participation in case of (history of) liver disease, (history of) excessive alcohol consumption (i.e., $>3$ and $>2$ units per day for males and females, respectively), major change in weight (i.e., $>5 \%$ ) and/or physical activity pattern in the three months prior to the study, use of glucose-lowering drugs, recent illness, pregnancy and/or lactation, contraindications for magnetic resonance imaging (MRI) and inability to give informed consent. Eligibility assessment was performed by the clinical researcher of the study (N.S.).

All participants gave written informed consent prior to inclusion in the study. The study was performed according to the Declaration of Helsinki ${ }^{16}$ and approved by the medical ethical committee of Maastricht University Medical Center. The full trial protocol can be accessed via the corresponding author or ClinicalTrials.gov (identifier: NCT03067428).

\section{Dietary intervention}

Since restriction of one nutrient (in this case fructose) without affecting other components of the diet is practically impossible, both groups were asked to follow a six-week fructose-restricted diet. In addition to this diet, the control group was supplemented with fructose powder aimed at achieving a fructose intake similar to baseline, whereas the intervention group remained fructoserestricted and received glucose powder to allow an isocaloric comparison.

Dietary intake was assessed with a three-day food journal along with a personal interview by the clinical researcher (N.S.) who was blinded to the intervention assignment. Weight of the food products was provided by the 
participant or estimated using average quantities per portion. Macro- and micronutrient composition and caloric content of the diet were calculated using the Dutch food composition table. ${ }^{17}$ Average fructose intake was calculated with an extensive database of food products from the Wageningen University. ${ }^{15}$ This database was also used for the composition of the fructose-restricted diet throughout the study. If the fructose content of a specific product was not available in the database, a comparable food product or the sucrose content $(=50 \%$ fructose $)$ of the specific product was used.

All participants received extensive counseling on the fructose-restricted diet by the clinical researcher of the study (N.S.) under the supervision of an experienced metabolic dietician (E.M.C.P.), who were both blinded to the intervention assignment. Participants were allowed to have an ad-libitum food intake, as long as the fructose intake per meal and per day was less than 7.5 grams and 10 grams, respectively. For this, participants were provided a list of permitted and prohibited food products as well as examples of fructoserestricted meals. Furthermore, participants received dietary counseling on a weekly basis. Substantial weight loss/gain was checked every week and (if necessary) corrected with dietary advice. Randomization to either glucose or fructose supplementation was computer-generated using block sizes of four and performed by an independent researcher (M.D.G.E.). The allocation sequence was concealed in an opaque, sealed envelope and remained blinded to the participants and assessors (N.S., P.I.H.G.S., M.C.G.J.B., C.D.A.S., N.C.S., P.V. and V.B.H.S.) upon completion of all analyses. Supplementation of either glucose or fructose equalled the amount of fructose that was restricted from the diet. In case of a baseline fructose intake below the Dutch average (i.e., 45 grams/day), glucose or fructose supplementation was set at $45 \mathrm{grams} / \mathrm{day}$. The glucose and fructose powders (indistinguishable in terms of color and odor) were prepacked in identical sachets by an independent researcher (M.D.G.E.) and distributed to the participants on a weekly basis. Participants were instructed to dissolve the glucose or fructose supplementation in water or food for consumption during the three main meals. As the six-week fructose-restricted diet is devoid of fruits and vegetables, all participants received vitamin $C$ supplementation $(70 \mathrm{mg} /$ day) throughout the entire study to prevent deficiency. No additional requirements were imposed in terms of food/liquid intake or medication use. Participants were asked not to alter their physical activity throughout the study. 


\section{Primary outcome measure}

Participants were asked to visit the research ward of Maastricht University Medical Center after an 8-hour fast and to refrain from alcohol 3 days prior to the measurements. Proton magnetic resonance spectroscopy ( $\left.{ }^{1} \mathrm{H}-\mathrm{MRS}\right)$ was performed to determine IHL content at baseline and completion. All magnetic resonance (MR) measurements were performed on a 3 Tesla MR system (Achieva 3T-X Philips Healthcare, Best, Netherlands) using a 32-channel sense cardiac/torso coil (Philips Healthcare, Best, Netherlands). Due to morbid (abdominal) obesity, five participants were scanned on a wide bore system 1.5 T MR system (Ingenia, Philips Healthcare, Best, Netherlands) at both time points (i.e., baseline and follow-up measurement). $\mathrm{A}_{2}$-weighted turbo spin echo MR image was acquired in three (axial, coronal and transversal) orientations and a $20 \times 20 \times 20 \mathrm{~mm}$ voxel was placed in the right hepatic lobe, avoiding vascular structures and edges of the liver, diaphragm and biliary structures. Special care was taken to ensure that the placement of the voxel was in same position at both time points. Hepatic lipid spectra were acquired using a PRESS sequence with water suppression (frequency selective prepulses) using the following MR parameters: repetition time (TR)/echo time (TE) $=4000 / 32.5 \mathrm{~ms}, 32$ acquisitions with 2 signal averages in each, bandwith $=2000$ $\mathrm{Hz}$ and data points $=2048$. Additionally, spectra without water suppression were acquired (8 acquisitions with 2 signal averages in each) as an internal reference. The long TR was chosen to let the participant breath in the rhythm of the measurement and MR acquisition was performed at the end of expiration. $A$ pressure-sensitive probe was placed on the abdomen of the participant to monitor the breathing pattern throughout the measurement. All MR spectra obtained were individually frequency-aligned and -phased, and lipid- $\mathrm{CH}_{2}$ and water peaks were fitted to the respective spectrum as described before ${ }^{18}$, using a custom-built MATLAB script (MATLAB R2017b, Mathworks, USA). The signal intensities obtained were corrected for $T_{2}$ decay using the literature $T_{2}$ values of 3T $(26.3 \mathrm{~ms}, 59.1 \mathrm{~ms})^{19}$ and $1.5 \mathrm{~T}(50 \mathrm{~ms}, 60 \mathrm{~ms})^{20}$ for water and lipid, respectively. Finally, the $\mathrm{IHL}$ percentage was represented as the area ratio of the $\mathrm{CH}_{2} / \mathrm{H}_{2} \mathrm{O}$ peak using the $\mathrm{T}_{2}$-corrected signal intensities of lipid- $\mathrm{CH}_{2}$ and water. Due to technical reasons, MRS was not available in one participant. IHL content of both time points was subsequently assessed using mDIXON imaging, which was validated against ${ }^{1} \mathrm{H}-\mathrm{MRS}$ in a former study $\left(\mathrm{ICC}_{\text {agreement }}\right.$ $=0.82 ; p<0.001^{21}$ ).

\section{Secondary outcome measures}

At the same day of the ${ }^{1} \mathrm{H}-\mathrm{MRS}$ measurements, blood was drawn for the determination of serum insulin and lipids (i.e., total, HDL and LDL cholesterol 
and triglycerides) and a two-hour 75-grams oral glucose tolerance test (OGTT) was performed, all exactly as described previously. ${ }^{22}$ The area under the curve (AUC) was calculated as a measure of glucose tolerance. Insulin resistance was estimated with the HOMA2-IR calculator (www.dtu.ox.ac.uk).

\section{Other measurements}

All participants filled in a health questionnaire concerning smoking, habitual alcohol consumption and medical history. Weight was measured in solely underwear, height was assessed with a stadiometer and waist and hip circumference were determined with a measuring tape at the level of umbilicus and trochanter major, respectively. 24-hour urine was collected in pre-acidified plastic containers at $\mathrm{T}=0,2,4$ and 6 weeks for measurement of urinary fructose, an objective biomarker of fructose consumption ${ }^{23}$, with Ultra Performance Liquid Chromatography-tandem Mass Spectrometry (UPLCMS/MS; VC\% 6.6).

\section{Statistical analysis}

For this double-blind randomized controlled trial, sample size calculation showed that 19 individuals per group were required to detect a mean difference of $3 \% \pm 3.2 \%$ in IHL content $\left(\alpha=0.05, \beta=0.20\right.$, allocation ratio $N_{\text {intervention group: }}$ : $\left.\mathrm{N}_{\text {control group }}=1: 1\right)$. To account for drop-out $(15 \%)$, the number of individuals was increased to 22 per group.

Dichotomous data are expressed as frequencies. Continuous data are presented as median (interquartile range). Changes from baseline within and between groups were analyzed with a Wilcoxon signed-rank test and a Mann Whitney $\mathrm{U}$ test, respectively. The $95 \%$ confidence intervals $(95 \% \mathrm{Cl})$ for these changes were calculated according to the Hodges-Lehmann method. Sensitivity analyses were conducted 1) to assess the influence of unbalanced randomization, if present, on the primary outcome measure. For this, a oneway ANCOVA was conducted with the unbalanced variable as a covariate; 2) to determine the effect of the protocol amendment (i.e., the omission of the fructose intake $\geq 45$ grams/day inclusion criterion) on the primary outcome measure; and 3) to assess the effect of the degree of IHL content at baseline (i.e., above or below the $5.56 \%$ cut-off value ${ }^{20}$ ) on the primary outcome measure. For both analyses, interaction terms were tested in a one-way ANCOVA. $\mathrm{P}<0.05$ was considered statistically significant. All analyses were carried out with the IBM Statistical Package of Social Sciences (SPSS) version 25 for Windows (SPSS inc. Chicago, IL). 


\section{Results}

\section{Randomization and follow-up}

Forty-four participants were randomly assigned to the intervention group (fructose-restricted diet plus glucose supplementation) or control group (fructose-restricted diet plus fructose supplementation) (see Figure 9.1 for flow chart). Six participants discontinued participation because of various reasons (i.e., claustrophobia during MR measurements $(n=2)$, non-adherence to the diet (as indicated by the participant) $(n=1)$, immobility due to ankle distortion $(n=1)$, symptoms related to irritable bowel syndrome (before start of the diet) $(n=1)$ and personal reasons $(n=1)$ ). At completion of the study, one participant did not appear to meet the inclusion criteria (i.e., FLI $\geq 60$ ) and was therefore excluded from the analyses. The individualized supplementation was successfully supplied and ingested by all participants. Statistical analyses were carried out according to an intention-to-treat principle with 16 and 21 participants in the intervention and control group, respectively. Baseline characteristics are displayed in Table 9.1.

Table 9.1 General baseline characteristics of the study population.

\begin{tabular}{lcc}
\hline Characteristic & Intervention group $(\mathrm{n}=16)$ & Control group $(\mathrm{n}=21)$ \\
\hline Age $(\mathrm{yr})$ & $55(35-62)$ & $52(38-62)$ \\
Sex $(\mathrm{n}, \mathrm{M} / \mathrm{F})$ & $6 / 10$ & $6 / 15$ \\
Smoking $\%$ yes) & 12.5 & 14.3 \\
Alcohol intake (units/week) & $2(1-5)$ & $3(0-5)$ \\
BMI $\left(\mathrm{kg} / \mathrm{m}^{2}\right)$ & $34.1(28.8-37.3)$ & $31.1(30.2-35.6)$ \\
Waist circumference (cm) & $117.9(106.5-128.4)$ & $110.0(104.3-113.6)$ \\
HOMA2-IR & $0.84(0.50-1.37)$ & $0.86(0.73-1.14)$ \\
Serum total cholesterol (mmol/L) & $5.2(4.3-5.6)$ & $5.2(4.2-6.0)$ \\
Serum HDL cholesterol (mmol/L) & $1.2(1.1-1.3)$ & $1.3(1.0-1.5)$ \\
Serum LDL cholesterol (mmol/L) & $3.1(2.3-3.8)$ & $2.7(2.3-3.8)$ \\
Serum triglycerides (mmol/L) & $1.6(1.2-1.9)$ & $1.3(0.9-1.7)$ \\
Dietary fructose intake (g/day) & $42.1(20.3-73.4)$ & $36.9(27.1-54.6)$ \\
Fatty liver index & $94(74-96)$ & $84(64-91)$ \\
Intrahepatic lipid content (\%) & $4.9(2.3-10.3)$ & $2.1(0.9-7.7)$ \\
\hline
\end{tabular}

Data are expressed as median (interquartile range). Abbreviations: years (yr), male (M), female (F), homeostasis model assessment of insulin resistance (HOMA2-IR), high-density lipoprotein (HDL), low-density lipoprotein (LDL). 
Enrollment

Assessed for eligibility $(n=71)$

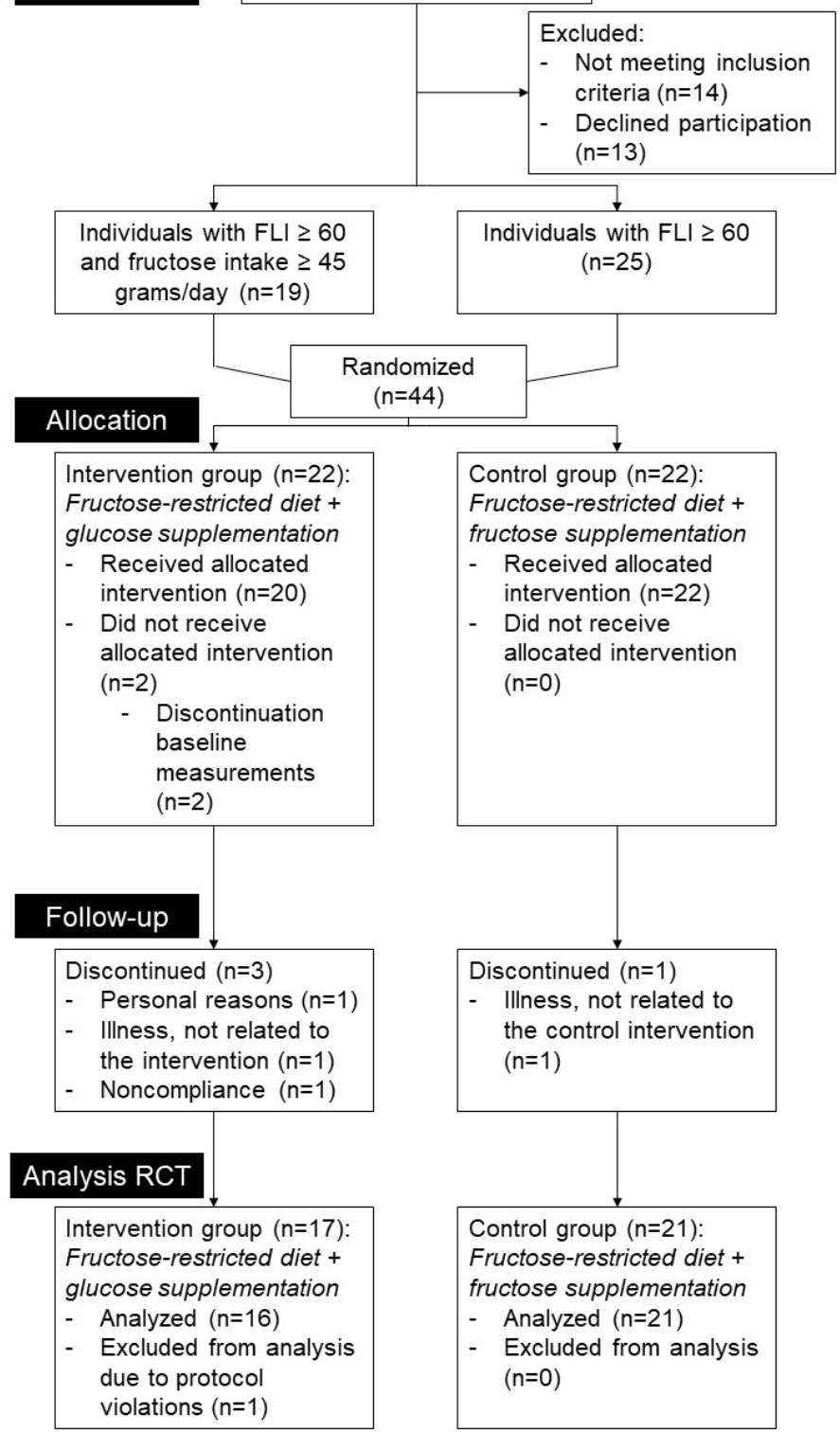

Figure 9.1 Flow chart of study. 


\section{Adherence and tolerability of the intervention}

Compared to baseline, the habitual dietary intake of fructose decreased drastically in both groups (Figure 9.2, panel A). The median dietary fructose intake after six weeks of fructose restriction was 1.4 grams/day in the intervention group and 2.2 grams/day in the control group (change from baseline: -45.7 grams/day, $95 \% \mathrm{Cl}$ : $-61.4 ;-29.8$ and -37.9 grams/day, $95 \% \mathrm{Cl}$ : $-48.3 ;-28.6$, respectively). The change from baseline was not statistically different between both groups (difference between change from baseline: -6.7 grams/day, $95 \% \mathrm{Cl}$ : $-24.0 ; 13.0)$. When the supplementation was taken into account, the fructose intake in the control group increased to 49.1 grams per day (change from baseline: +12.4 grams/day, $95 \% \mathrm{Cl}: 6.2 ; 20.8$ ). This was higher than baseline, since supplementation of fructose (and glucose) was set at 45 grams/day in all participants with a baseline fructose intake below the Dutch average (see Methods section). This change was significantly different from the intervention group (difference between change from baseline: -57.0 grams/day, $95 \% \mathrm{Cl}$ : $-39.5 ;-77.9$; Figure 9.2 , panel $\mathrm{B}$ ). The cumulative difference in fructose intake during the full study period - calculated as the individual difference from baseline times the number of days each participant was included in the study - was 1,894 grams $(95 \% \mathrm{Cl}: 1,727 ; 2,052)$. This difference was also reflected by a statistically different urinary fructose excretion at completion of the study (Figure 9.2, panel C).

Caloric intake remained stable throughout the intervention as a consequence of isocaloric supplementation and did not differ between the two groups (Figure 9.2, panel D). Nevertheless, BMl decreased in both the intervention and control group (change from baseline: $-0.2 \mathrm{~kg} / \mathrm{m}^{2}, 95 \% \mathrm{Cl}$ : $-0.5 ; 0.0$ and $-0.4 \mathrm{~kg} / \mathrm{m}^{2}$, $95 \% \mathrm{Cl}:-0.6 ;-0.1$, respectively), which was not different between both groups (difference between change from baseline: $-0.1 \mathrm{~kg} / \mathrm{m}^{2}, 95 \% \mathrm{Cl}:-0.3 ; 0.5$; Figure 9.2, panel E). No statistically significant effect was observed for macronutrient composition (i.e., carbohydrate, protein, total fat and saturated fat intake; Supplementary Figure S9.1, panel A-D). When macronutrient composition was expressed as percentage of total daily energy intake, a significant difference between change from baseline was observed for carbohydrate intake (difference between change from baseline: $7.1 \%, 95 \% \mathrm{Cl}$ : $0.7 ; 14.3$; Supplementary Figure S9.2, panel A), which appeared to be in exchange for protein intake (difference between change from baseline: $-5.9 \%, 95 \% \mathrm{Cl}$ : -13.3;0.4; Supplementary Figure S9.2, panel B).

The intervention was generally well tolerated. Diarrhea (grade 1-2) was reported in three and five participants in the intervention and control group, 
respectively. Only one participant in the control group experienced a serious adverse event, which occurred between the screening visit and baseline measurements.

A

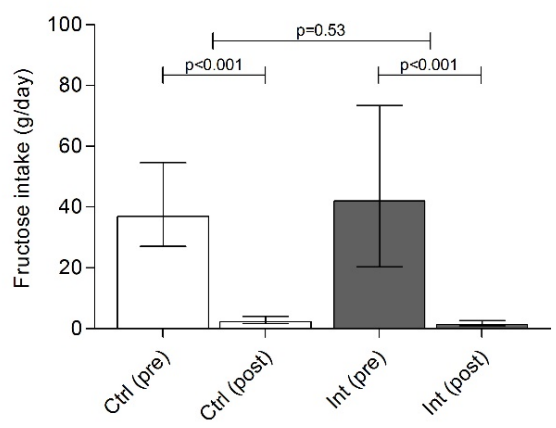

\section{B}

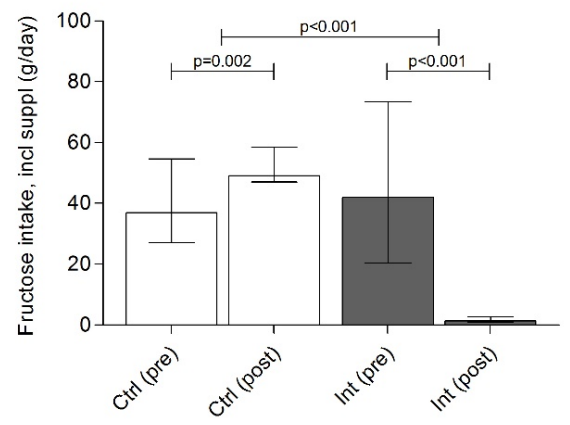

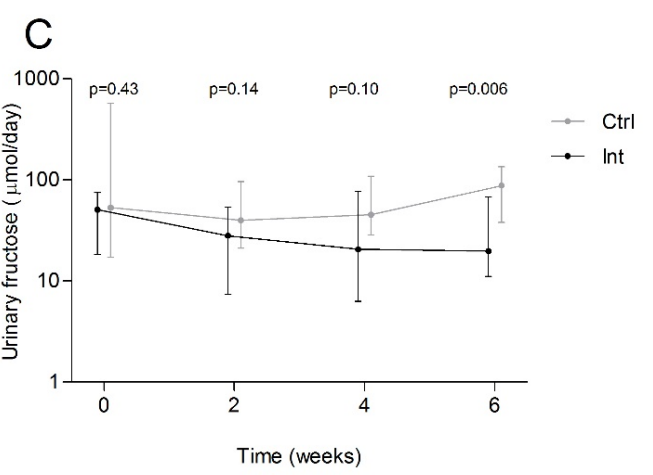
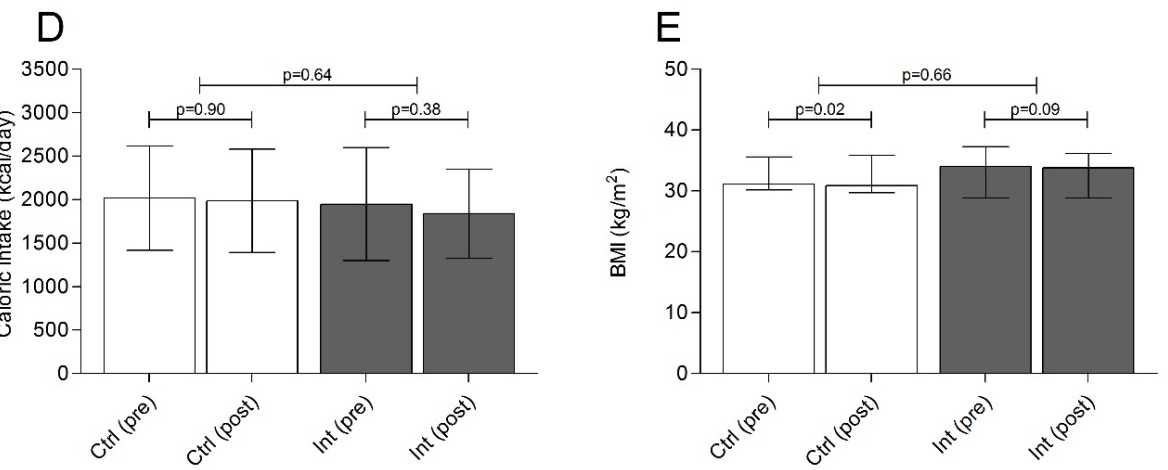

Figure 9.2 Adherence to the intervention. Daily fructose intake (A), daily fructose intake including supplementation (B), 24-hour urinary fructose concentration (C), daily caloric intake (D) and body mass index (BMI) (E) in the control (white bars) and intervention (grey bars) group at baseline (pre) and after completion of the study (post). Data are expressed as median (interquartile range). Differences within groups are analyzed with a Wilcoxon signed-rank test. Differences between groups are analyzed with a Mann Whitney $U$ test. 


\section{Effect of fructose restriction on intrahepatic lipid content}

IHL content decreased in both treatment arms (Figure 9.3, panel A). The reduction in $\mathrm{IHL}$ content was significantly greater in the intervention group, albeit small (difference between change from baseline: $-0.7 \%, 95 \% \mathrm{Cl}$ : -2.0;-0.03; Figure 9.3, panel A). Since baseline BMI and IHL content tended to be higher in the intervention group (Table 9.1), we subsequently conducted a sensitivity analysis to assess the impact thereof on the primary outcome measure. Addition of baseline BMI and IHL as covariates to a one-way ANCOVA did not have substantial effects on the (log-transformed) primary outcome measure (beta: $-0.06,95 \% \mathrm{Cl}$ : $-0.116 ; 0.007, \mathrm{p}=0.08$ ). Subgroup analyses did not show different effect sizes between participants with either high or low habitual fructose intake, or high or low baseline IHL content ( $p$ for interaction $=0.63$ and 0.75 , respectively; Supplementary Figure S9.3).

\section{Effect of fructose restriction on glucose tolerance and serum lipid levels}

Plasma glucose excursions during a two-hour 75-grams OGTT were not different between the two groups (difference between change from baseline: 32.3, 95\% Cl: -62.3;119.0; Figure 9.3, panel B). HOMA2-IR was neither affected in the intervention nor in the control group (change from baseline: $0.12,95 \% \mathrm{Cl}$ : $-0.17 ; 0.51$ and $0.06,95 \% \mathrm{Cl}:-0.13 ; 0.19$, respectively), nor was there a difference between the groups (difference between change from baseline: 0.10 , 95\% Cl: $-0.21 ; 0.42)$. Additionally, no effects were observed on serum lipids (Figure 9.3, panel C-F). 
A
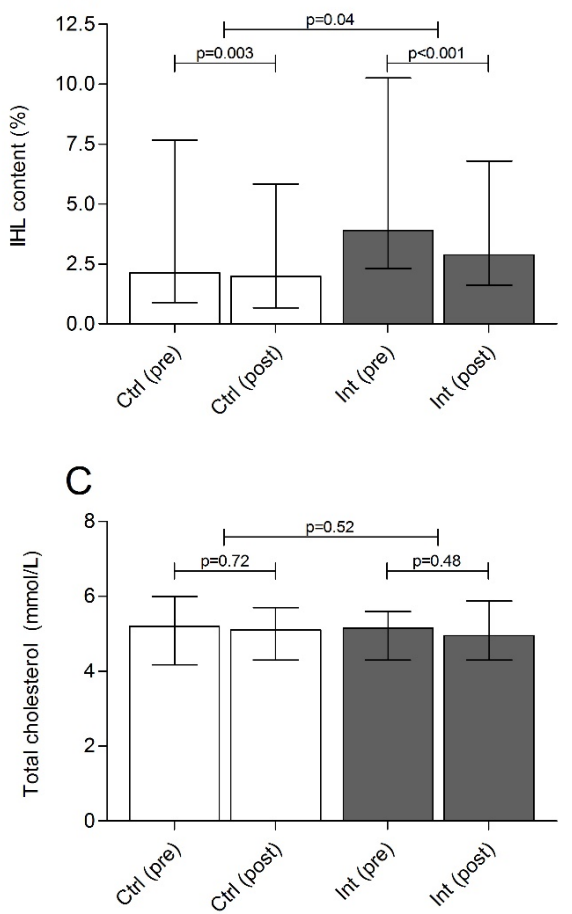

E

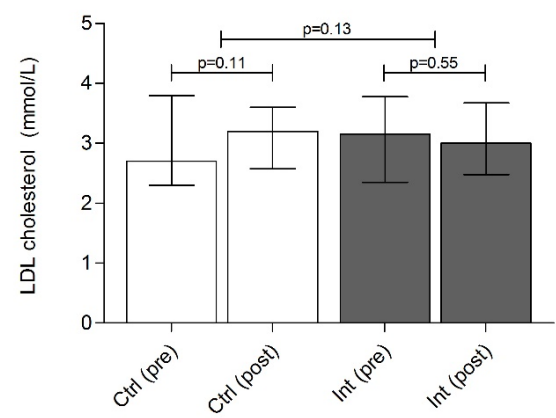

B
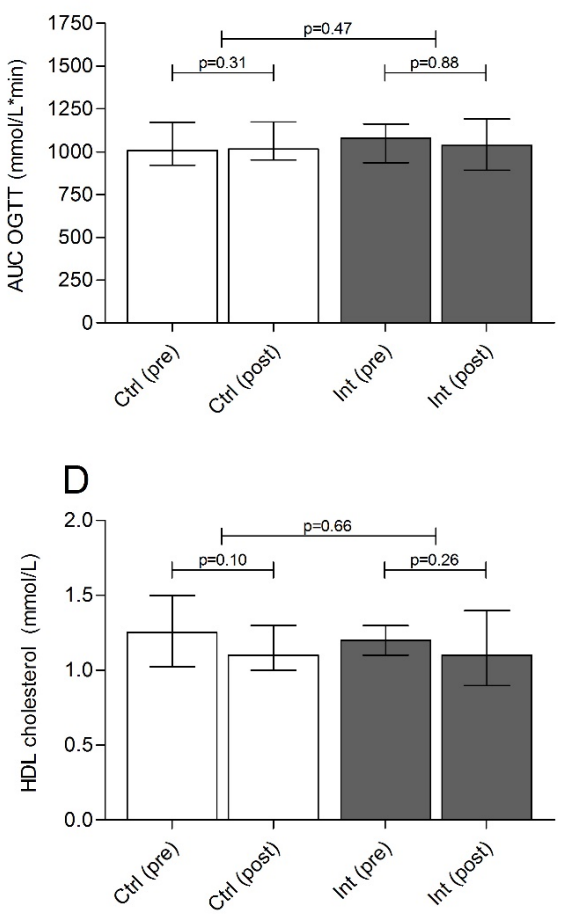

$\mathrm{F}$

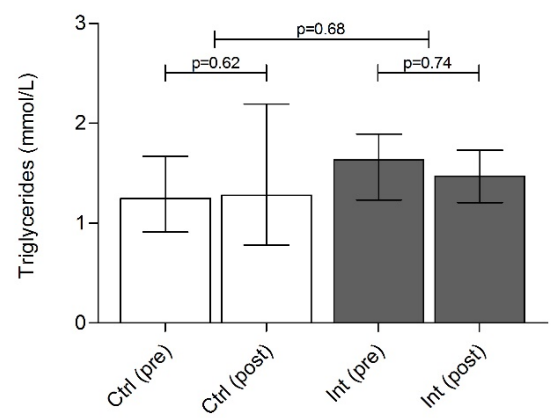

Figure 9.3 Intrahepatic lipid content, glucose tolerance and serum lipid levels. Intrahepatic lipid content (A), glucose area under the curve during an oral glucose tolerance test (B), serum total cholesterol (C), serum HDL cholesterol (D), serum LDL cholesterol $(E)$ and serum triglycerides $(F)$ in the control (white bars) and intervention (grey bars) group at baseline (pre) and after completion of the study (post). Data are expressed as median (interquartile range). Differences within groups are analyzed with a Wilcoxon signed-rank test. Differences between groups are analyzed with a Mann Whitney U test. 


\section{Discussion}

In this double-blind randomized controlled trial, we show that a six-week fructose-restricted diet resulted in a small decrease in IHL content in adult overweight individuals with a high $\mathrm{FLI}$ in comparison to an isocaloric comparator. No effects were observed on glucose tolerance or serum lipid levels.

Although glucose and fructose are both simple sugars with identical molecular formulas, their metabolic fate differs greatly. In contrast to glucose, which is widely metabolized throughout the body, a proportion of ingested fructose is already cleared in the small intestine, as shown by animal studies. ${ }^{24}$ After absorption, fructose reaches the portal blood where it is almost entirely taken up by the liver to be converted to glucose, lactate, glycogen or triglycerides. ${ }^{25}$ Furthermore, at a low dose, fructose facilitates hepatic glucose disposal by dissociation of the glucokinase-glucokinase regulatory protein complex. ${ }^{11,26}$ Dissociation of this complex appears clinically relevant, since carriers of a missense variant in GCKR - the gene that encodes a glucokinase regulatory protein that binds glucokinase less effectively - are prone to develop NAFLD. ${ }^{27}$ Thus, although clear metabolic differences between fructose and glucose metabolism exist, such differences cannot be easily extrapolated to net effects on metabolic health, including $\mathrm{IHL}$ content, and experiments such as the current trial may provide important insights.

Dose-dependent metabolic pathways as described above may also explain the conflicting results of previous clinical trials addressing the role of fructose in intrahepatic lipid accumulation. ${ }^{5,8}$ To date, fructose intervention studies have mainly focused on the role of fructose added to the diet and showed contrasting effects on IHL content. ${ }^{5}$ Recently, Schwarz and colleagues showed that restriction of fructose from the diet reduced IHL content in obese children and adolescents. The lack of a control group, however, makes it difficult to conclude whether this change in IHL content can be attributed to fructose restriction per se, or alternatively, to the change in the diet in general or other behaviors that are associated with participation in a clinical trial (i.e., the Hawthorne effect ${ }^{28}$ ).

The current study is the first that used a randomized controlled study design in which both the intervention and control group followed the same fructoserestricted diet. Together with a similar caloric intake in both treatment arms (as a consequence of the isocaloric glucose supplementation in the intervention group), we were able to study the effect of fructose restriction per se. Indeed, 
the results in the control group show that the diet by itself was already associated with a decrease in IHL content, independent of a change in fructose intake (which was supplemented). More importantly, by comparing both treatment arms we are able to show that fructose restriction per se reduces IHL content, albeit with a small effect size ( 0.7 percent point).

In the present study, we did not observe any effect of fructose restriction on serum lipid levels, glucose tolerance or HOMA2-IR, which was somewhat surprising since these variables have been associated with $\mathrm{IHL}$ content. ${ }^{29,30} \mathrm{~A}$ possible explanation might be that the effect of fructose restriction on IHL content was too small to result in a detectable change in serum lipid levels and/or glucose tolerance. In addition, we did not assess insulin sensitivity with the gold standard method, i.e., hyperinsulinemic-euglycemic clamp. Another possible explanation might be that the duration of the intervention was too short. Yet, this period is comparable to most other fructose feeding trials, which varied between the three and ten weeks. ${ }^{31}$ Moreover, it has been shown that $\mathrm{IHL}$ accumulation can resolve within days. ${ }^{32} \mathrm{We}$, therefore, believe that a sixweek intervention period - in which the cumulative difference in fructose consumption was almost 2 kilograms - is sufficient to reach a steady state in these metabolic outcomes.

We also believe that diets without fructose such as the one we employed should not be used in daily practice because the observed effects on IHL content were small and, hence, not clinically relevant. Furthermore, such diets not only are devoid of processed foods, but also of fruits and vegetables. The latter are widely accepted as healthy food products because of their high fiber and vitamin content. Indeed, it has been postulated that the presence of vitamin $C$ in fruit and vegetables abolishes the toxic effects of fructose. ${ }^{33-35}$ In our study, both treatment arms received vitamin $C$ throughout the study, which might have resulted in an underestimation of the actual effect of fructose restriction per se.

Our study has strengths and limitations. First, the sample size was relatively small, but should be viewed in perspective of the major dietary restrictions that were imposed. More importantly, our study was adequately powered with an expected drop-out rate. Second, we used the FLI as a screening tool for NAFLD. ${ }^{36}$ Despite a high positive likelihood ratio to rule in NAFLD with a $\mathrm{FLI} \geq 60^{37-39}$, the median $\mathrm{IHL}$ content of the study participants was only $3.4 \%$. This was not attributable to regression to the mean, since FLI was high at both the screening and baseline visit (91 versus 87 , respectively). Nevertheless, sensitivity analysis with stratification for high and low IHL content showed 
comparable effect sizes in individuals with a high $\mathrm{IHL}$ content at baseline. Third, due to a slow inclusion rate we decided to abandon the inclusion criterion of fructose intake $\geq 45$ grams/day. To safeguard a detectable effect on $\mathrm{IHL}$ content, the supplementation of either glucose or fructose was increased to 45 grams/day in participants with a low habitual fructose intake. Again, a sensitivity analysis did not show any statistical difference when we stratified for fructose intake at baseline. Fourth, despite major efforts to achieve an isocaloric diet in comparison to the habitual baseline diet of participants, BMI decreased similarly in both the intervention and control group, which could be explained by the Hawthorne effect. A major strength of this study is the doubleblind randomized controlled design, using an isocaloric comparator. As hitherto mentioned, both groups followed the same fructose-restricted diet, which is of importance, given the differential impact of macronutrients on IHL content. ${ }^{40,41}$ Despite this design, we did find some small, probably accidental differences in carbohydrate intake expressed as percentage of total energy intake (i.e., relatively higher in the intervention group), which tended to be in exchange for protein intake (i.e., relatively lower in the intervention group). Given the previously described effects of carbohydrates and proteins on IHL content (i.e., predisposing and protective, respectively $)^{40,41}$, the effects of these subtle differences in macronutrient composition on $\mathrm{IHL}$ content, if any, have probably mitigated the observed difference in the current study. Finally, not only did we used a three-day food journal combined with personal interviews to assess fructose intake throughout the study, we were also able to objectively confirm adherence to the study protocol by measuring urinary fructose levels, a biomarker of fructose intake. ${ }^{23}$

In conclusion, in this double-blind randomized controlled trial, we showed that a six-week fructose-restricted diet results in a small but statistically significant decrease in IHL content in overweight adult individuals with a high FLI. No effects were observed on glucose tolerance or serum lipid levels. 


\section{Literature}

1. Bray GA, Nielsen SJ, Popkin BM. Consumption of high-fructose corn syrup in beverages may play a role in the epidemic of obesity. Am J Clin Nutr 2004;79:537-43.

2. Schulze MB, Manson JE, Ludwig DS, et al. Sugar-sweetened beverages, weight gain, and incidence of type 2 diabetes in young and middle-aged women. JAMA 2004;292:927-34.

3. Jensen T, Abdelmalek MF, Sullivan $S$, et al. Fructose and sugar: A major mediator of nonalcoholic fatty liver disease. J Hepatol 2018;68:1063-75.

4. Yang Q, Zhang Z, Gregg EW, Flanders WD, Merritt R, Hu FB. Added sugar intake and cardiovascular diseases mortality among US adults. JAMA Intern Med 2014;174:516-24.

5. Chiu S, Sievenpiper JL, de Souza RJ, et al. Effect of fructose on markers of non-alcoholic fatty liver disease (NAFLD): a systematic review and meta-analysis of controlled feeding trials. European journal of clinical nutrition 2014;68:416-23.

6. Hannou SA, Haslam DE, McKeown NM, Herman MA. Fructose metabolism and metabolic disease. J Clin Invest 2018;128:545-55.

7. Sievenpiper JL, de Souza RJ, Jenkins DJ. Sugar: fruit fructose is still healthy. Nature 2012; 482:470.

8. Schwarz JM, Noworolski SM, Erkin-Cakmak A, et al. Effects of Dietary Fructose Restriction on Liver Fat, De Novo Lipogenesis, and Insulin Kinetics in Children With Obesity. Gastroenterology 2017;153:743-52.

9. Gluchowski NL, Becuwe M, Walther TC, Farese RV, Jr. Lipid droplets and liver disease: from basic biology to clinical implications. Nat Rev Gastroenterol Hepatol 2017;14:343-55.

10. DiStefano JK. Fructose-mediated effects on gene expression and epigenetic mechanisms associated with NAFLD pathogenesis. Cell Mol Life Sci 2020;77(11):2079-90.

11. Petersen KF, Laurent D, Yu C, Cline GW, Shulman GI. Stimulating effects of low-dose fructose on insulin-stimulated hepatic glycogen synthesis in humans. Diabetes 2001;50: 1263-8.

12. Buziau AM, Schalkwijk CG, Stehouwer CDA, Tolan DR, Brouwers M. Recent advances in the pathogenesis of hereditary fructose intolerance: implications for its treatment and the understanding of fructose-induced non-alcoholic fatty liver disease. Cell Mol Life Sci 2020;77(9):1709-19.

13. Li L, Liu DW, Yan HY, Wang ZY, Zhao SH, Wang B. Obesity is an independent risk factor for non-alcoholic fatty liver disease: evidence from a meta-analysis of 21 cohort studies. Obes Rev 2016;17:510-9.

14. Bedogni G, Bellentani S, Miglioli L, et al. The Fatty Liver Index: a simple and accurate predictor of hepatic steatosis in the general population. BMC Gastroenterol 2006;6:33.

15. Sluik D, Engelen Al, Feskens EJ. Fructose consumption in the Netherlands: the Dutch National Food Consumption Survey 2007-2010. Eur J Clin Nutr 2015;69:475-81.

16. World Medical Association Declaration of Helsinki: ethical principles for medical research involving human subjects. JAMA 2013;310:2191-4.

17. Rijksinstituut voor Volksgezondheid en Milieu (National Institute for Public Health and the Environment) (2010) NEVO-tabel (Dutch Food Composition Table). Nederlands Voedingsstoffenbestand 2010 (Dutch Food Composition Database 2010). Den Haag: RIVM.

18. Lindeboom L, Nabuurs $\mathrm{Cl}$, Hesselink MK, Wildberger JE, Schrauwen P, SchrauwenHinderling VB. Proton magnetic resonance spectroscopy reveals increased hepatic lipid content after a single high-fat meal with no additional modulation by added protein. Am J Clin Nutr 2015;101:65-71.

19. Guiu B, Petit JM, Loffroy R, et al. Quantification of liver fat content: comparison of triple-echo chemical shift gradient-echo imaging and in vivo proton MR spectroscopy. Radiology 2009;250:95-102. 
20. Szczepaniak LS, Nurenberg $P$, Leonard D, et al. Magnetic resonance spectroscopy to measure hepatic triglyceride content: prevalence of hepatic steatosis in the general population. Am J Physiol Endocrinol Metab 2005;288:E462-8.

21. Kusters YH, Schalkwijk CG, Houben AJ, et al. Independent tissue contributors to obesityassociated insulin resistance. JCl insight 2017;2(13):e89695.

22. Simons N, Debray FG, Schaper NC, et al. Patients with aldolase B deficiency are characterized by an increased intrahepatic triglyceride content. J Clin Endocrinol Metab 2019;104(11):5056-64.

23. Tasevska N, Runswick SA, McTaggart A, Bingham SA. Urinary sucrose and fructose as biomarkers for sugar consumption. Cancer Epidemiol Biomarkers Prev 2005;14:1287-94.

24. Jang C, Hui S, Lu W, et al. The Small Intestine Converts Dietary Fructose into Glucose and Organic Acids. Cell Metab 2018;27:351-61.e3.

25. Sun SZ, Empie MW. Fructose metabolism in humans - what isotopic tracer studies tell us. Nutr Metab 2012;9:89.

26. Shiota $M$, Moore $M C$, Galassetti $P$, et al. Inclusion of low amounts of fructose with an intraduodenal glucose load markedly reduces postprandial hyperglycemia and hyperinsulinemia in the conscious dog. Diabetes 2002;51:469-78.

27. Speliotes EK, Yerges-Armstrong LM, Wu J, et al. Genome-wide association analysis identifies variants associated with nonalcoholic fatty liver disease that have distinct effects on metabolic traits. PLoS Genet 2011;7:e1001324.

28. McCarney R, Warner J, lliffe S, van Haselen R, Griffin M, Fisher P. The Hawthorne Effect: a randomised, controlled trial. BMC Med Res Methodol 2007;7:30.

29. DeFilippis AP, Blaha MJ, Martin SS, et al. Nonalcoholic fatty liver disease and serum lipoproteins: the Multi-Ethnic Study of Atherosclerosis. Atherosclerosis 2013;227:429-36.

30. Wang B, Li M, Zhao Z, et al. Glycemic measures and development and resolution of nonalcoholic fatty liver disease in nondiabetic individuals. J Clin Endocrinol Metab 2020.

31. Sievenpiper JL, de Souza RJ, Cozma Al, Chiavaroli L, Ha V, Mirrahimi A. Fructose vs. glucose and metabolism: do the metabolic differences matter? Curr Opin Lipidol 2014;25: 8-19.

32. Bortolotti M, Kreis R, Debard $\mathrm{C}$, et al. High protein intake reduces intrahepatocellular lipid deposition in humans. Am J Clin Nutr 2009;90:1002-10.

33. Lanaspa MA, Sanchez-Lozada LG, Choi YJ, et al. Uric acid induces hepatic steatosis by generation of mitochondrial oxidative stress: potential role in fructose-dependent and independent fatty liver. J CBiol Chem 2012;287:40732-44.

34. Kc S, Carcamo JM, Golde DW. Vitamin C enters mitochondria via facilitative glucose transporter 1 (Glut1) and confers mitochondrial protection against oxidative injury. FASEB J 2005;19:1657-67.

35. Johnson RJ, Stenvinkel P, Andrews $\mathrm{P}$, et al. Fructose metabolism as a common evolutionary pathway of survival associated with climate change, food shortage and droughts. J Intern Med 2020;287:252-62.

36. EASL-EASD-EASO Clinical Practice Guidelines for the management of non-alcoholic fatty liver disease. J Hepatol 2016;64:1388-402.

37. Yang $\mathrm{BL}, \mathrm{Wu} \mathrm{WC}$, Fang $\mathrm{KC}$, et al. External validation of fatty liver index for identifying ultrasonographic fatty liver in a large-scale cross-sectional study in Taiwan. PLoS One 2015;10:e0120443.

38. Cuthbertson DJ, Weickert MO, Lythgoe D, et al. External validation of the fatty liver index and lipid accumulation product indices, using $1 \mathrm{H}$-magnetic resonance spectroscopy, to identify hepatic steatosis in healthy controls and obese, insulin-resistant individuals. Eur J Endocrinol 2014;171:561-9.

39. Koehler EM, Schouten JN, Hansen BE, Hofman A, Stricker BH, Janssen HL. External validation of the fatty liver index for identifying nonalcoholic fatty liver disease in a populationbased study. Clin Gastroenterol Hepatol 2013;11:1201-4. 
40. Luukkonen PK, Sadevirta S, Zhou Y, et al. Saturated Fat Is More Metabolically Harmful for the Human Liver Than Unsaturated Fat or Simple Sugars. Diabetes Care 2018;41:1732-9.

41. Markova M, Pivovarova O, Hornemann S, et al. Isocaloric Diets High in Animal or Plant Protein Reduce Liver Fat and Inflammation in Individuals With Type 2 Diabetes. Gastroenterology 2017;152:571-85.e8. 


\section{Supplemental materials}
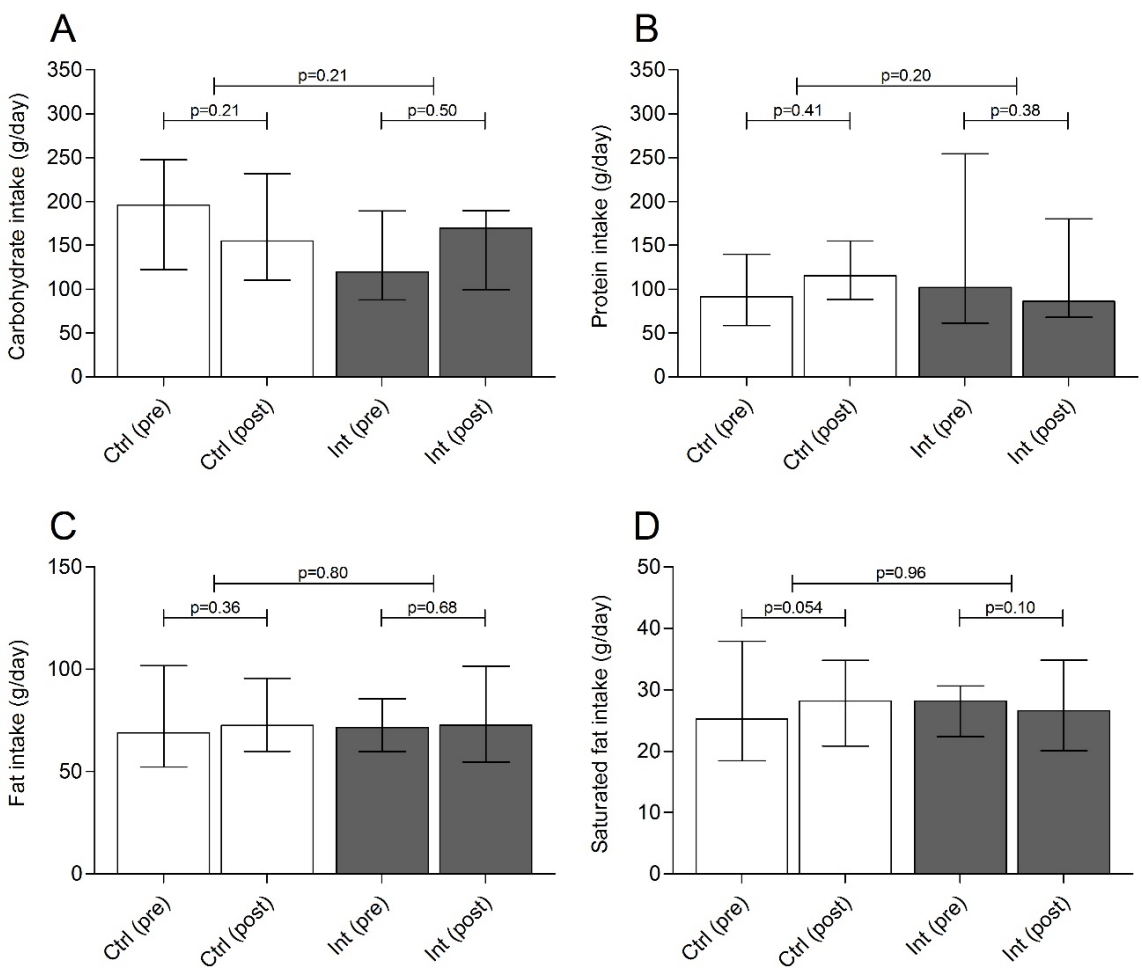

Figure S9.1 Macronutrient composition pre- and post-intervention. Daily carbohydrate (A), protein (B), total fat $(C)$ and saturated fat $(D)$ intake in the control (white bars) and intervention (grey bars) group at baseline (pre) and after completion of the study (post). Data are expressed as median (interquartile range). Differences within groups are analyzed with a Wilcoxon signed-rank test. Differences between groups are analyzed with a Mann Whitney $U$ test. 

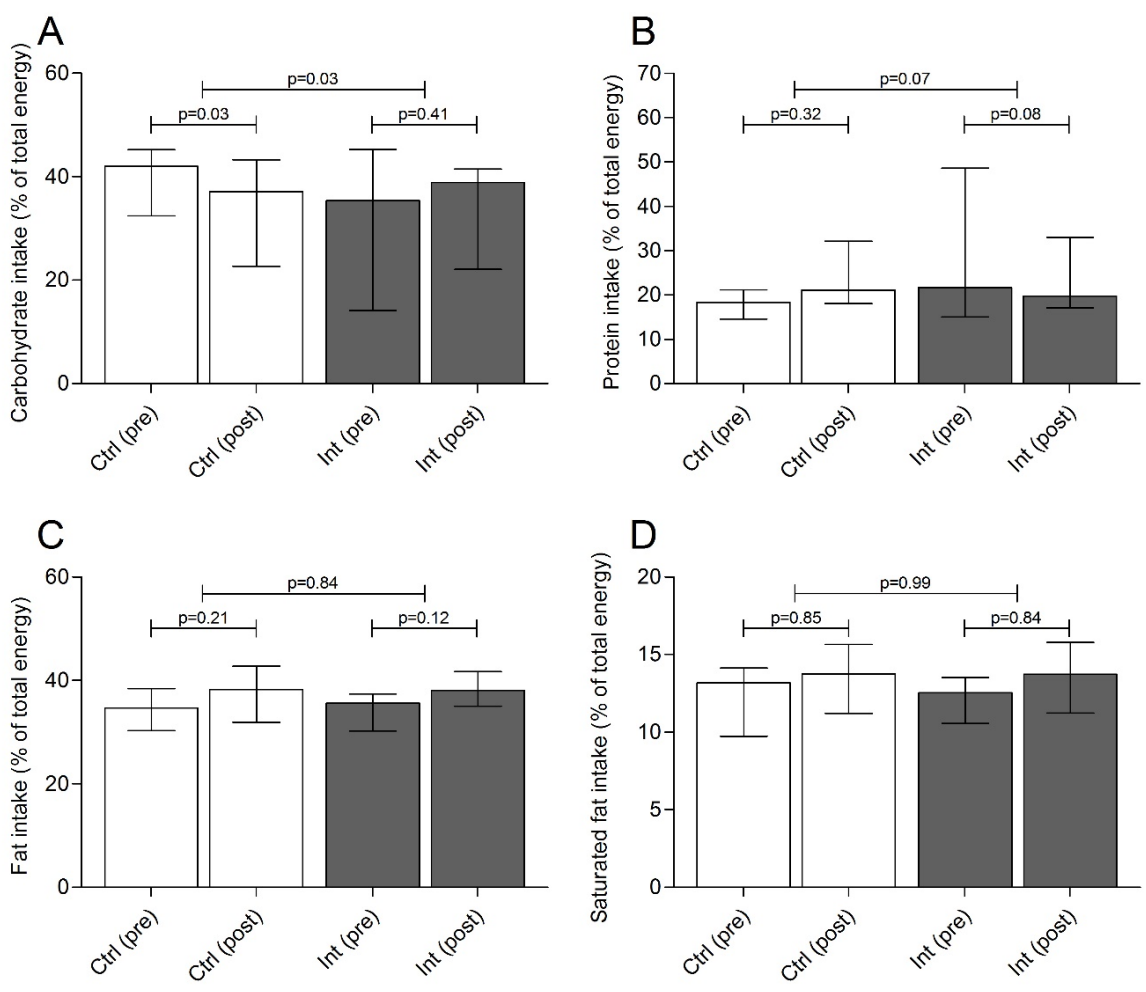

Figure S9.2 Macronutrient composition expressed as percentage of total energy intake pre- and postintervention. Daily carbohydrate (A), protein (B), total fat (C) and saturated fat (D) intake expressed as percentage of daily energy intake in the control (white bars) and intervention (grey bars) group at baseline (pre) and after completion of the study (post). Data are expressed as median (interquartile range). Differences within groups are analyzed with a Wilcoxon signed-rank test. Differences between groups are analyzed with a Mann Whitney $U$ test.

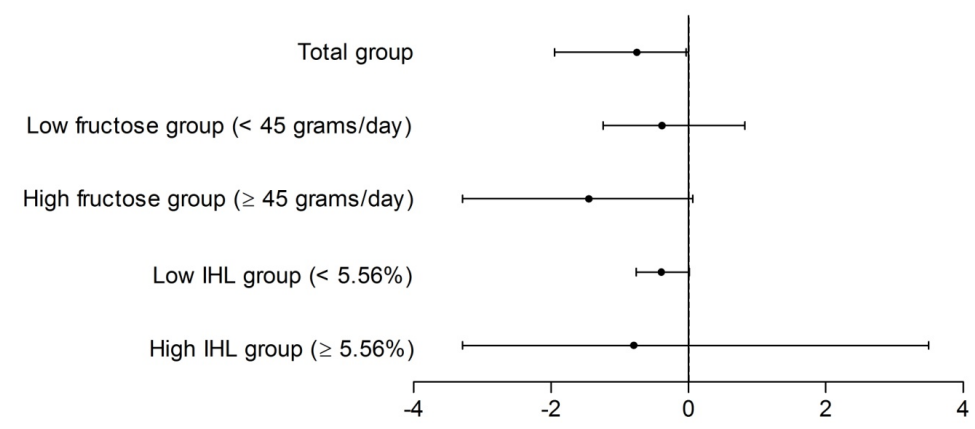

Difference in change in IHL content (\%)

Figure S9.3 Subgroup analyses based on fructose intake and IHL content at baseline. 



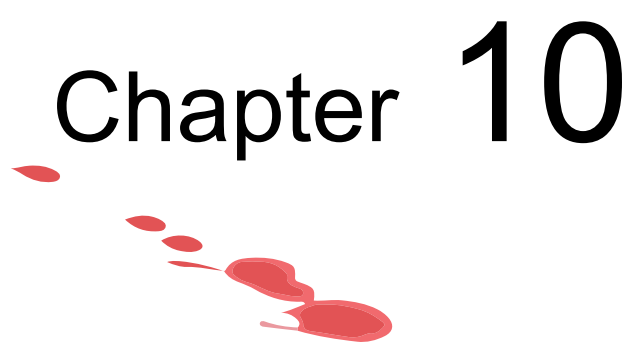

\section{Summary and general discussion}
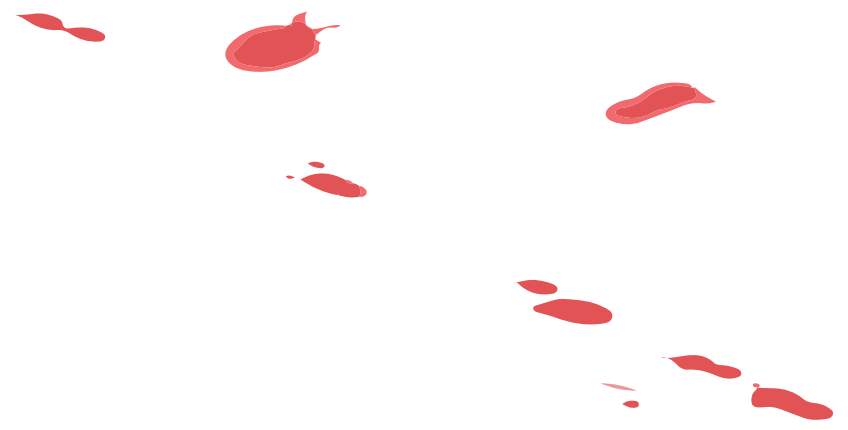

Parts of this chapter have been published as:

Nonalcoholic fatty liver disease and cardiovascular disease:

assessing the evidence for causality

Martijn C.G.J. Brouwers, Nynke Simons, Coen D.A. Stehouwer, 
Chapter 10 


\section{Key findings and discussion}

Cardiovascular disease (CVD) is a major threat to morbidity and mortality worldwide. ${ }^{1}$ In the last decade much attention has been paid to the role of nonalcoholic fatty liver disease (NAFLD) in the development of CVD. Although several high quality meta-analyses identified NAFLD as a risk factor $^{2-4}$, is remains unclear whether NAFLD is an active contributor to cardiovascular risk or just an innocent bystander.

In the current thesis, we have tried to unravel the relationship between NAFLD and CVD, and possible underlying mechanisms, by using a combination of genetic epidemiology, experimental mice and human biopsy data, as well as case-control and randomized controlled studies.

This chapter summarizes and discusses the key findings of the thesis in light of current scientific knowledge. In addition, methodological considerations of the performed studies, as well as possible clinical applications and future research generated by the results, will be addressed.

\subsection{Part I. Genetic studies to elucidate the relationship between intrahepatic lipid content and CVD}

\subsubsection{Key findings}

The objective of the first part of this thesis was to assess the presence of a causal relationship between NAFLD and CVD in humans. A second objective was to determine if the relationship between NAFLD and CVD depends on the pathway that leads to intrahepatic lipid (IHL) accumulation, the first stage of NAFLD. Since the implementation of stable isotopes to study specific pathways in vivo in a large number of participants is practically impossible, we used genetic epidemiology as an alternative approach. Currently, four genetic variants have been associated with all stages of NAFLD. ${ }^{5}$ The mechanisms by which these genetic variants predispose to NAFLD differs: rs738409 (PNPLA3) and rs58542926 (TM6SF2) result in IHL accumulation via decreased production and secretion of triglyceride-rich VLDL particles ${ }^{6,7}$, whereas rs641738 (MBOAT7) and rs1260326 (GCKR) lead to impaired mobilization of triglycerides ${ }^{8}$ and increased de novo lipogenesis ${ }^{9}$, respectively. By studying the associations of these genetic variants with coronary artery disease (CAD) in several large cohorts (chapter three and four), we found that individuals carrying the rs1260326 T-allele (GCKR) displayed a higher risk of CAD, 
whereas individuals carrying the rs738409 G-allele (PNPLA3) and rs58542926 T-allele (TM6SF2) were protected against CAD. No effect on CAD risk was observed for the rs641738 T-allele (MBOAT7). In addition, the rs1260326 T-allele $(G C K R)$ was associated with a more atherogenic lipid profile in individuals with type 2 diabetes compared to individuals with an impaired and normal glucose metabolism (chapter two). Last, when all currently reported NAFLD susceptibility genes $(n=12)$ were clustered, no effect on CAD risk was observed. Subsequent analyses revealed that a substantial part of the heterogeneity of the effects of the individual genes on CAD risk could be explained by the effects of these genes on plasma lipids. Furthermore, when genetic variants that are implicated in the development of NAFLD through impaired secretion of triglyceride-rich VLDL particles were excluded, a positive relationship with CAD was observed (chapter five).

Based on the results of these genetic epidemiology studies, we conclude that NAFLD per se does not cause CVD. The association between NAFLD and CVD depends on the pathway that leads to $\mathrm{HH}$ accumulation, in which pathways that result in increased secretion of triglyceride-rich VLDL particles (and hence a more atherogenic lipid profile) - such as de novo lipogenesis are deleterious for cardiovascular health.

\subsubsection{Discussion}

\subsubsection{Interaction between variants in GCKR and environmental factors}

In chapter two, we observed that rs1260326 (GCKR) interacts with glucose metabolism status on plasma triglyceride and HDL cholesterol levels. There are several other studies that corroborate the observed interaction of GCKR with environmental factors. First, Orho-Melander and colleagues reported that rs780094 (which is in strong linkage disequilibrium with rs1260326 $(G C K R)^{10}$ ) was associated with increased plasma triglycerides. ${ }^{10}$ Interestingly, the difference in plasma levels between carriers and non-carriers was much larger in individuals with type 2 diabetes $^{10}$ in comparison to the general population. ${ }^{11}$ Furthermore, Cole and colleagues found an interaction between rs1260326 $(G C K R)$ and BMI on plasma lipids. ${ }^{12}$ We failed to replicate this finding, although this might be due to lack of statistical power as the $p$ for interaction was 0.13 . Finally, two studies have shown that rs780094 (GCKR) interacts with BMI and insulin resistance on $\mathrm{IHL}$ content ${ }^{13,14}$, which is believed to drive the overproduction of triglyceride-rich VLDL particles resulting in high plasma triglyceride levels. ${ }^{15}$ These gene-environment interactions demonstrate that the effects of a common genetic variant, which is generally small, can become 
more pronounced and clinically relevant when present in a metabolically unhealthy environment. Unfortunately, in our systematic review and metaanalysis (chapter three) we were not able to collect sufficient data on BMI, glucose metabolism status or insulin resistance to test for an interaction with the rs1260326 T-allele (GCKR) on CAD, estimated glomerular filtration rate (eGFR) and chronic kidney disease (CKD).

\subsubsection{Effects of variants in GCKR on glomerular filtration rate}

In addition to the increased risk of CAD, the rs1260326 T-allele (GCKR) was also associated with an increased eGFR and tended to protect from CKD (chapter three). This finding is somewhat surprising given the fact that NAFLD has been reported as a risk factor for CKD in some epidemiological studies. ${ }^{16-18}$ One explanation could be that another gene that is in linkage disequilibrium with rs1260326 (GCKR), is actually responsible for the association with glomerular function. However, previous experiments in liver-specific glucokinase knockout mice (which are metabolically opposite to rs1260326 $(G C K R))$ are characterized by increased kidney damage ${ }^{19}$, which is in line with our findings. The exact mechanism by which the rs 1260326 T-allele (GCKR) exerts its renoprotective effects is not entirely clear. The rs1260326 T-allele $(G C K R)$ has been associated with factors predisposing to CKD (i.e., NAFLD, low HDL cholesterol levels and high urate levels ${ }^{10,20-23}$ ) and factors protecting from CKD (i.e., lower plasma glucose levels ${ }^{10,24,25}$ ). Further studies are required to elucidate the mediating factor of this apparent hepatorenal axis.

\subsubsection{Relationship between NAFLD and CVD}

The findings reported in part $\mathrm{I}$ of this thesis corroborate previous epidemiological studies. In three meta-analyses ${ }^{2-4}$ NAFLD (assessed by imaging or histology) was associated with increased (fatal and non-fatal) cardiovascular events. In contrast, a recently published retrospective casecontrol study based on four electronic primary care databases (containing data of approximately 17.7 million European individuals) reported no association between NAFLD and acute myocardial infarction, after adjustment for traditional cardiovascular risk factors. ${ }^{26}$ It should be noted that some of these risk factors are not necessarily confounders, but actual mediators of the relationship between NAFLD and CVD (as outlined in paragraph 4 of the Introduction). Moreover, in spite of the large study population, the diagnosis of NAFLD was based on electronic medical records only, which increases the risk of underestimation of exposure (=non-differential misclassification) and, hence, bias towards the null. ${ }^{27}$ Indeed, the prevalence of NAFLD in this study was 
much lower (i.e., 1.9\%) compared to the world wide prevalence of NAFLD $(\sim 25 \%)^{28}$ and studies using imaging or histology as diagnostic tool $(36.6 \%){ }^{4}$

Unfortunately, due to the observational design of epidemiological studies, no conclusions can be made regarding the causal effect of NAFLD on CVD. The Mendelian randomization approach, however, allows to make causal inferences in observational study designs. Since an individual is randomized at conception to receive an allele that either protects or predisposes to NAFLD, conclusions regarding a causal relationship with CVD can be drawn. Based on the results of the meta-analysis described in chapter three, it can be concluded that NAFLD - more specifically increased de novo lipogenesis - is causally related to CAD. Indeed, the rs1260326 T-allele (GCKR) encodes a liver-specific protein that has been associated with increased de novo lipogenesis ${ }^{29}$ and NAFLD. ${ }^{20}$ The protective relationship between the rs738409 (PNPLA3) and rs58542926 (TM6SF2) variants (both involved in the production and secretion of triglyceride-rich VLDL particles) and CAD (chapter four), the positive association between the NAFLD susceptibility genes and CAD after exclusion of genes involved in triglyceride-rich VLDL secretion (including PNPLA3 and TM6SF2) (chapter five), and the positive association between plasma lipids and CAD conferred by 12 NAFLD susceptibility genes (chapter five) all hint towards a mediating effect of plasma lipids on the relationship between NAFLD and CAD. Other mediators that have been proposed as factors connecting NAFLD with CVD, such as low-grade inflammation (which is present in both NASH and atherosclerosis), seem less important as most of the genetic variants studied (e.g., rs738409 (PNPLA3), rs58542926 (TM6SF2), rs1260326 (GCKR) and rs641738 T-allele (MBOAT7)) have been associated with all histological stages of NAFLD, including NASH, but have different effects on CAD risk. Moreover, in chapter five, the intercept of the relationship between plasma lipids and CAD risk conferred by NAFLD susceptibility genes included zero, implicating that if the NAFLD susceptibility genes have no effect on plasma lipids then the risk of CAD is also neutral. Type 2 diabetes - another suggested mediator for the association between NAFLD and CVD - also appears less important since both rs738409 (PNPLA3) and rs58542926 (TM6SF2) predispose to type 2 diabetes $^{30}$, but protect from CAD (chapter four). 


\subsection{Part II Relationship between NAFLD and biomarkers of endothelial function}

\subsubsection{Key findings}

In Part I of this thesis we found that the cardiovascular risk is different for individuals with NAFLD caused by decreased production and secretion of triglyceride-rich VLDL particles and individuals with NAFLD caused by increased de novo lipogenesis. In epidemiological studies, plasma biomarkers of endothelial (dys)function (for example soluble endothelium selectin (sE-selectin) and soluble vascular cell adhesion molecule 1 (sVCAM)) have often been used to assess cardiovascular risk. ${ }^{31-33}$ On the other hand, previous experimental studies have shown that E-selectin is involved in the pathogenesis of alcoholic fatty liver disease, which histologically resembles NAFLD, via the induction of hepatic neutrophil infiltration. ${ }^{34}$ Therefore, the objective of the second part if this thesis was to study the individual relationship between the different biochemical pathways leading to $\mathrm{IHL}$ accumulation (i.e., rs738409 (PNPLA3) and rs1260326 (GCKR)) and these biomarkers. For this we used data from two prospective cohorts designed to study determinants and (cardiovascular) complications of type 2 diabetes. We found that both rs1260326 (GCKR) and rs738409 (PNPLA3) were associated with higher plasma sE-selectin levels (but not with sVCAM levels), despite their opposite relationships with plasma triglycerides levels, type 2 diabetes and CAD risk (as shown in part I of this thesis). Based on these outcomes and the hitherto mentioned study in an animal model of alcoholic fatty liver disease ${ }^{34}$, we hypothesized a role for E-selectin in the pathophysiology of NAFLD and, hence, the liver endothelium as a source of circulating sE-selectin (which could explain the observed association between both NAFLD susceptibility genes and plasma sE-selectin levels). For this, we used data from experimental mice and human biopsy studies and indeed found that plasma sE-selectin was associated with NAFLD severity and hepatic E-selectin expression. In addition, plasma ALT (used as a marker of liver parenchyma damage) was associated with plasma sE-selectin, independent of potential confounders. Such consistent associations were not observed for sVCAM.

Based on the results of these genetic epidemiology and experimental mice and human biopsy studies, we conclude that E-selectin is involved in the pathophysiology of NAFLD and plasma sE-selectin might originate from the steatotic/inflamed liver. 


\subsubsection{Discussion}

\subsubsection{1. sE-selectin as a biomarker of hepatic endothelial function}

Although the experimental mouse and human biopsy studies in part II of this thesis were merely observational, the positive association of both rs 1260326 (GCKR) and rs738409 (PNPLA3) - two NAFLD susceptibility genes with opposite effects on cardiovascular risk - with sE-selectin, is suggestive of a causal relationship between NAFLD per se (independent of the underlying mechanism) and sE-selectin. Although we were not able to differentiate between sE-selectin derived from the liver and from other organs, there are other studies that support the concept of the fatty/inflamed liver as a source of circulating sE-selectin. A study in mice, for example, showed active involvement of liver sinusoidal endothelial cells in the pathogenesis of NAFLD. ${ }^{35}$ In addition, hepatic endothelial dysfunction is already present in the earliest stage of NAFLD development. ${ }^{36,37}$

The findings of part II of this thesis puts previous epidemiological studies, in which plasma sE-selectin was associated with cardiometabolic outcomes, in another perspective. Of all plasma biomarkers of endothelial dysfunction, sEselectin was the strongest predictor of new-onset type 2 diabetes. ${ }^{31,38} \mathrm{~A}$ recent meta-analysis demonstrated that NAFLD is a risk factor for type 2 diabetes. $^{39}$ The results of this thesis, therefore, suggest that hepatic endothelial dysfunction (as part of NAFLD) is involved in the pathogenesis of type 2 diabetes. Although the precise mechanism is not clear, previous experimental animal studies have shown that the endothelium of a fat-loaded liver is resistant to insulin. ${ }^{40}$

\subsection{Part III Modulation of fructose metabolism to study the relationship between NAFLD and CVD}

\subsubsection{Key findings}

The objective of the third part of this thesis was to study the cardiometabolic effects of fructose-mediated dissociation of glucokinase from glucokinase regulatory protein (and, consequently, increased de novo lipogenesis) in humans. For this, we assessed IHL content and cardiovascular risk factors in patients with hereditary fructose intolerance $(\mathrm{HFI})$, a rare metabolic disorder characterized by an impaired breakdown of fructose-1-phosphate due to aldolase $\mathrm{B}$ deficiency ${ }^{41}$, and in overweight individuals on a fructose-restricted diet (the FRUITLESS study). 
We found that in overweight individuals with an elevated fatty liver index, six weeks of fructose restriction led to a decrease in IHL content. No effect was observed on glucose metabolism or plasma lipid levels (chapter nine). In patients with $\mathrm{HFI}, \mathrm{IHL}$ content, hypoglycosylated transferrin levels (a surrogate marker for hepatic fructose-1-phosphate concentrations) and glucose levels during an oral glucose tolerance test were higher when compared to age-, sexand BMI-matched controls (chapter seven). In addition, HFI patients were characterized by a higher eGFR, systolic blood pressure and sE-selectin level (chapter eight).

Based on the results of these clinical studies, we conclude that fructose (specifically an intermediate of fructose metabolism, i.e., fructose-1-phosphate) contributes to IHL accumulation, which suggests a possible role of glucokinase regulatory protein.

\subsubsection{Discussion}

\subsubsection{Fructose and NAFLD}

The results from previous clinical studies on the relationship between fructose intake and NAFLD development have been conflicting. A recent meta-analysis did not demonstrate any change in IHL content after dietary interventions with added fructose. ${ }^{42}$ Clinical trials that have shown a change in IHL accumulation often used a fructose overload in combination with a hypercaloric diet. ${ }^{43-46}$ In contrast, Schwarz and colleagues showed that a 9-day isocaloric fructoserestricted diet successfully reduced the IHL content in obese children with high habitual sugar intake. ${ }^{47}$ This study, however, was greatly limited by the lack of a control group, which makes it difficult to conclude whether the change in IHL content was caused by fructose restriction per se, the change in diet in general or the Hawthorne effect. ${ }^{48}$ In chapter nine, we demonstrated that fructose restriction per se decreases IHL content in adult overweight individuals with a high fatty liver index in comparison to an isocaloric comparator (i.e., glucose). The discrepancy with previous studies assessing the effect of fructose on NAFLD development may be attributed to the amount of fructose that was consumed (high versus low). Experimental studies have shown that fructose not only serves as a substrate for de novo lipogenesis and induces lipogenic transcription factors (such as sterol regulatory element binding protein 1 , carbohydrate response element binding protein and liver $X$ receptor $)^{49,50}$, but also increases hepatic glucose uptake at even small amounts (Figure 10.1). One putative mechanism is an enhanced disruption of the glucokinaseglucokinase regulatory protein complex (with similar metabolic consequences 
as the rs1260326 T-allele $(G C K R))^{51,52}$ (Figure 10.1). The findings in chapter seven are supportive of a prominent role for this complex in fructose-induced $\mathrm{IHL}$ accumulation. We found that patients with $\mathrm{HFI}$ - who are not capable of metabolizing fructose due to a genetic defect in aldolase $\mathrm{B}$ - are characterized by more hypoglycosylated transferrin (a surrogate marker for hepatic fructose1-phosphate concentrations, which is a potent disruptor of the glucokinaseglucokinase regulatory protein complex) and a higher IHL content. Of interest, these findings were recently confirmed in a mouse model of HFI that was characterized by an abundance of glucokinase in the cytosolic space, indicative of increased glucokinase-glucokinase regulatory protein dissociation. ${ }^{53}$

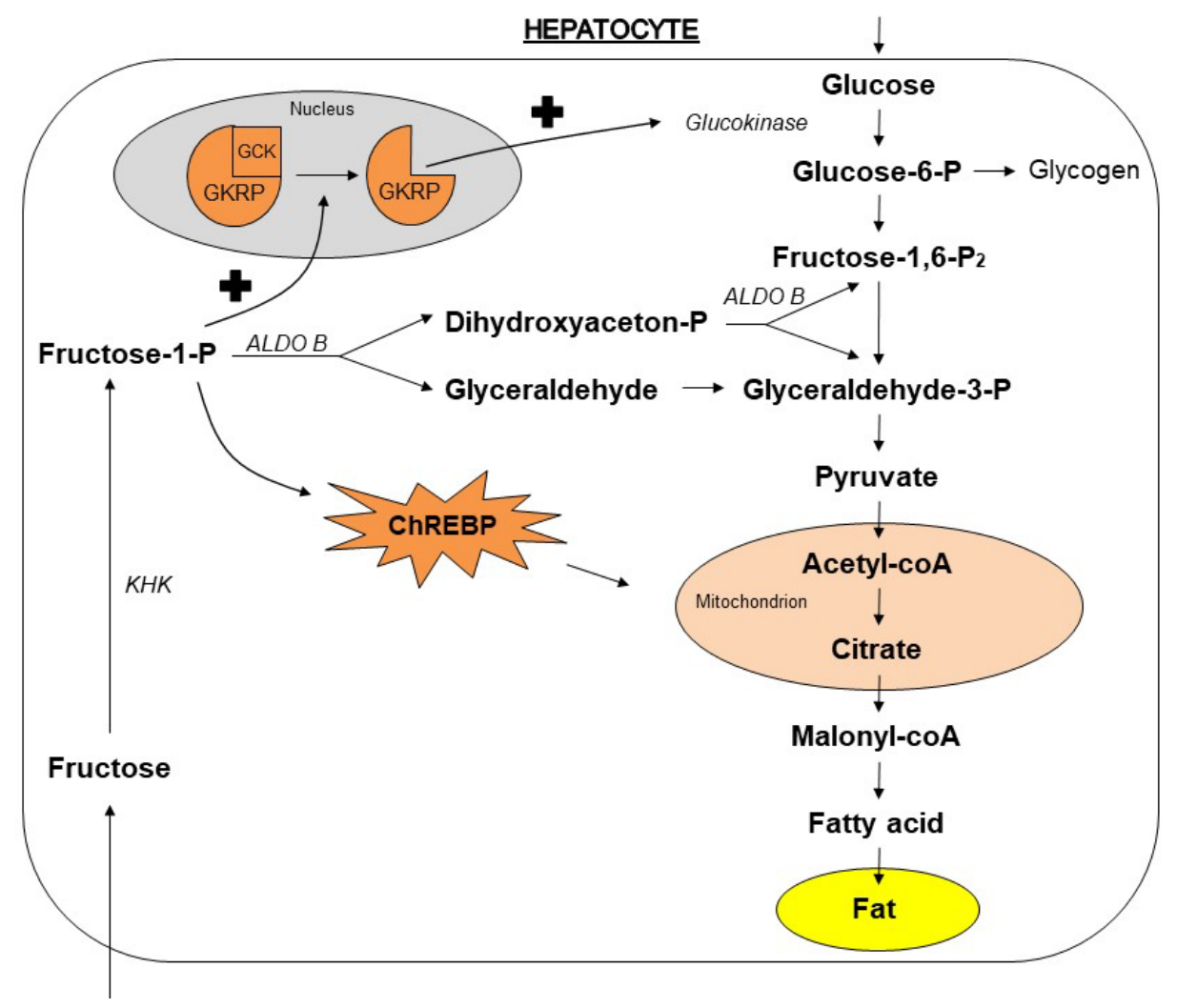

Figure 10.1 Role of fructose in intrahepatic lipid accumulation. Simplified schematic overview of the role of glucokinase regulatory protein and fructose in hepatic glycolysis and de novo lipogenesis. Figure adapted from Brouwers, Trends Mol Med (2015). Abbreviations: glucose-6-phosphate (glucose-6$P$ ), fructose-1,6-diphosphate (fructose-1,6- $P_{2}$ ), glyceraldehyde-3-phosphate (glyceraldehyde-3-P), acetyl coenzyme A (acetyl-coA), malonyl coenzyme A (malonyl-coA), fructose-1-phosphate (fructose-1-P), aldolase B (ALDO B) dihydroxyaceton-phosphate (dihydroxyaceton-P), glucokinase regulatory protein (GKRP), glucokinase (GCK), carbohydrate-responsive element-binding protein (ChREBP), ketohexokinase (KHK). 


\subsubsection{Fructose modulation to study the relationship between NAFLD and} CVD

In spite of a significant difference in IHL content, we did not find a difference in plasma lipids - the principal mediator of the relationship between NAFLD and CVD (part I of this thesis) - in both patients with HFI and individuals adhering to a six-week fructose-restricted diet (chapter seven and nine). This is surprising, since an increased $\mathrm{IHL}$ content has been associated with overproduction of triglyceride-rich VLDL particles. ${ }^{15}$ Furthermore, enhanced dissociation of the glucokinase-glucokinase regulatory protein complex - the presumed mechanism responsible for the differences in IHL content in our studies - has been associated with higher plasma triglycerides (chapter two). Several arguments can be put forward to account for this discrepancy: 1) As the genetic defect in patients with HFI is primarily located in the liver (and gut and kidney) with the remainder organs metabolically healthy, it is possible that these patients are solely characterized by increased production of plasma lipids which is compensated by an increased clearance by peripheral tissues. This explanation fits with the observations in chapter two where we found that the effects of enhanced glucokinase-glucokinase regulatory protein dissociation on plasma lipids is more pronounced when glucose metabolism deteriorates. Unfortunately, we have not performed stable isotope studies to quantify triglyceride-rich VLDL production in either study; 2) In both patients with HFI and participants of the FRUITLESS study the (change in) IHL content was still relatively low (albeit significantly different from controls) and probably too small to result in a (detectable) change in plasma lipid levels.

The small groups (and hence lack of statistical power) might explain why we did not observe a difference in markers of endothelial dysfunction and artery stiffness in patients with HFI, with the exception of plasma sE-selectin (chapter eight). Based on our findings in chapter six of this thesis, it is conceivable that the high sE-selectin levels in $\mathrm{HFI}$ patients are liver-derived due to $\mathrm{IHL}$ accumulation.

The higher eGFR that was observed in patients with HFI could reflect glomerular hyperfiltration (as seen in other metabolic conditions ${ }^{54}$ ), which is a precursor of CKD. ${ }^{55}$ Patients with HFI indeed had a higher dietary protein intake and blood pressure and lower glucose tolerance (chapter seven), which are all associated with glomerular hyperfiltration. ${ }^{56-58}$ On the other hand, a higher eGFR could also represent a better kidney function. In line with this (and discussed in paragraph 1.1.2.2.), individuals carrying the rs 1260326 T-allele 
(GCKR) also displayed a higher eGFR and tended to be protected against CKD. Although the metabolic consequences of the rs1260326 (GCKR) genetic variant and aldolase B deficiency are believed to be similar, a so-called hepatorenal axis as an underlying mechanism of the relationship between increased hepatic glycolysis/de novo lipogenesis and glomerular filtration needs further study.

\section{Methodological considerations}

The significance of the key findings of this thesis needs to be considered in light of the methodology by which they were generated. Part I consists of genetic epidemiology, in which cross-sectional associations were studied with genetic variants to make causal inferences. This Mendelian randomization approach has several assumptions as well as potential pitfalls. Second, casecontrol studies (part III) are suitable for the study of rare diseases (such as $\mathrm{HFI}$ ), but have some limitations in terms of external validity. Last, quality of data from randomized controlled trials (part III) relies for a large part on the accuracy of and adherence to the interventions that are used. These three main concerns will be discussed below.

\subsection{Assumptions, risk of pleiotropy and power in Mendelian randomization studies}

\subsubsection{Assumptions}

Mendelian randomization is a widely accepted approach to make causal inferences regarding the relationship between exposure and outcome. This approach is based on the principle that the human genome contains many genetic variations and that these so-called single nucleotide polymorphisms (SNPs) are randomly distributed to future haploid cells during meiosis. This lack of selection and undisputed beginning point makes SNP's useful to study causal relationships in cross-sectional study designs. For example, although CRP levels have consistently been associated with $C A D^{59-61}$, individuals carrying genetic variants that affect CRP levels do not have an increased risk of $C A D^{62}$, which makes CRP merely a biomarker, not a causal factor in the development of CAD.

There are, however, assumptions that must be met before conducting a Mendelian randomization study. First, the genetic variant must be associated with the risk factor of interest. Second, the genetic variant must not be 
associated with confounding factors. Last, the genetic variant must not be associated with the outcome of interest via other pathways. ${ }^{63,64}$ In genetic association studies, there are three situations in which the latter two assumptions are violated: (1) in case of pleiotropy, the genetic variant also affects other risk factors of the outcome; (2) in case of linkage disequilibrium, the genetic variant co-segregates with neighboring genes that affect the outcome; and (3) in case of population stratification, the frequency of the genetic variant is different among the different subpopulations used. ${ }^{63,64}$

\subsubsection{Pleiotropy}

As the liver is a highly active metabolic organ, there is a great risk of pleiotropy when studying NAFLD as the exposure of interest. Within pleiotropy, one can differentiate between horizontal (confounding) and vertical (mediation) pleiotropy, in which the latter does not violate the assumptions of Mendelian randomization $^{63,64}$ (Figure 10.2).

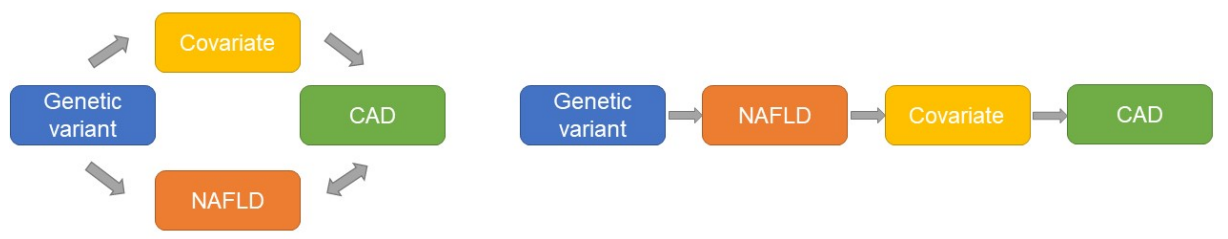

Figure 10.2 Schematic overview of horizontal (left panel) and vertical (right panel) pleiotropy in Mendelian randomization. Schematic overview of horizontal (left panel) and vertical (right panel) pleiotropy in Mendelian randomization. In case of horizontal pleiotropy, the genetic variant affects both the covariate and NAFLD. The covariate is causally related (unidirectional arrow) with CAD, whereas NAFLD is associated (bidirectional arrow) with CAD. In case of vertical pleiotropy, the genetic variant affects NAFLD and the covariate is a direct effect of NAFLD. The covariate is causally related with CAD (unidirectional arrow). Figure adapted from Brouwers, Diabetologia (2020). Abbreviations: nonalcoholic fatty liver disease (NAFLD), coronary artery disease (CAD).

In chapter four, we observed that the NAFLD susceptibility genes rs738409 (PNPLA3) and rs58542926 (TM6SF2) conferred a (modest) protection from $\mathrm{CAD}$, which is explained by their inhibiting effect on the production and secretion of triglyceride-rich VLDL particles (paragraph 1.1.2.3) The simultaneous effects of rs738409 (PNPLA3) and rs58542926 (TM6SF2) on both NAFLD and plasma lipids are an example of horizontal pleiotropy (Figure 10.3).

In chapter three, we observed that genetic variants in GCKR - that is involved in de novo lipogenesis, one of the principal pathways in the development of 
NAFLD ${ }^{65}$ - were modestly associated with CAD. Of interest, these genetic variants have also been associated with higher plasma triglycerides, lower plasma HDL cholesterol and the presence of small-dense LDL particles ${ }^{66}$, the lipid phenotype that characterizes NAFLD. ${ }^{67}$ Since it is believed that this lipid phenotype is a consequence of NAFLD ${ }^{66}$, it is an example of vertical pleiotropy, which does not violate the MR assumptions (Figure 10.3). However, it cannot be ruled out that the genetic variants in GCKR have horizontal pleiotropic effects as well, since they also protect from CKD (chapter three) and type 2 diabetes. $^{68,69}$

On the other hand, pleiotropy not necessarily limits the use of genetic variants in genetic epidemiology. In chapter six we used the presence of pleiotropy in rs1260326 (GCKR) and rs738409 (PNPLA3) to disentangle NAFLD from dyslipidemia, type 2 diabetes and CAD, and showed that sE-selectin is (causally) related to NAFLD.

Of course, the presence of horizontal pleiotropy is not limited to genetic studies using common variants (i.e., SNPs), it can also occur in genetic studies using rare mutations. In chapter seven we found that patients with HFI were characterized by a greater IHL content in comparison to age-, sex- and BMImatched controls. Besides an enhanced dissociation of the glucokinaseglucokinase regulatory protein complex, the extreme fructose-restricted diet patients with $\mathrm{HFI}$ need to adhere to could be an alternative explanation for the greater IHL content. Indeed, HFI patients consumed more saturated fat and protein, which both have been associated with $\mathrm{IHL}$ accumulation (albeit in different directions) ${ }^{70,71}$ Notably, statistical adjustment for protein and saturated fat consumption did not affect the outcomes. More importantly, IHL content was also higher in aldolase B knockout mice in comparison to wildtype mice at a background of fructose restriction in both groups. ${ }^{53}$ 


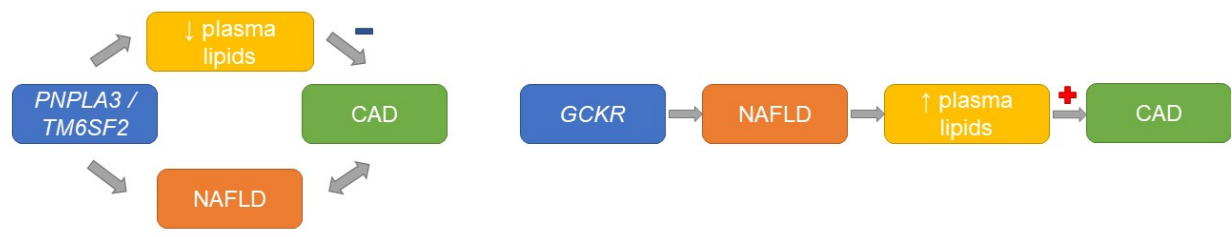

Figure 10.3 Schematic overview of horizontal and vertical pleiotropy in Mendelian randomization, using PNPLA3, TM6SF2 and GCKR to study the relationship between NAFLD and CAD. Schematic overview of horizontal (left panel) and vertical (right panel) pleiotropy in Mendelian randomization, using rs738409 (PNPLA3), rs58542926 (TM6SF2) and rs1260326 (GCKR) to study the relationship between NAFLD and CAD. Rs738409 (PNPLA3) and rs58542926 (TM6SF2) lead to decreased production and secretion of triglyceride-rich VLDL particles (and hence lower plasma lipid levels) as well as NAFLD development. As lower plasma lipid levels are causally related with CAD and NAFLD is associated with CAD, this is an example of horizontal pleiotropy and can be considered as confounding. Rs1260326 (GCKR), however, leads to NAFLD development, which subsequently causes higher plasma lipid levels. This is an example of vertical pleiotropy and can be considered as mediation. Figure adapted from Brouwers, Diabetologia (2020). Abbreviations: patatin-like phospholipase domain-containing protein 3 gene (PNPLA3), transmembrane 6 superfamily member 2 gene (TM6SF2), nonalcoholic fatty liver disease (NAFLD), coronary artery disease (CAD), glucokinase regulatory protein gene (GCKR).

\subsubsection{Statistical power}

It is a rule of thumb that the effect size of a genetic variant is inversely related to the allele frequency (Figure 10.4) and, hence, the effect sizes of common genetic variants are generally small. ${ }^{72}$ Therefore, to ensure sufficient statistical power to detect a significant effect, large study populations are required. Unfortunately, large study populations in which the genetic variant and exposure as well as the outcome of interest have been assessed are often not available. This can be overcome by combining (summarized) data from different cohorts, one containing gene-exposure data and the other geneoutcome data. The CARDIoGRAMplusC4D dataset is a well-known, publicly available dataset containing gene-CAD data of 60,801 cases and 123,504 controls. $^{73}$ Unfortunately, no such dataset exists for gene-NAFLD data. Therefore, in chapter three, four and five we were only capable of estimating the risk of CAD conferred by one-unit increase in NAFLD risk allele, instead of the risk of CAD conferred by one-unit increase in NAFLD (or IHL content).

On the other hand, effect sizes of rare variants, i.e., mutations, are generally large $^{72}$ (Figure 10.4). Paradoxically, the issue of statistical power is comparable to common variants: given the low prevalence it was difficult to collect sufficient cases with HFI, which explains - at least in part - some of the negative results in our studies. 


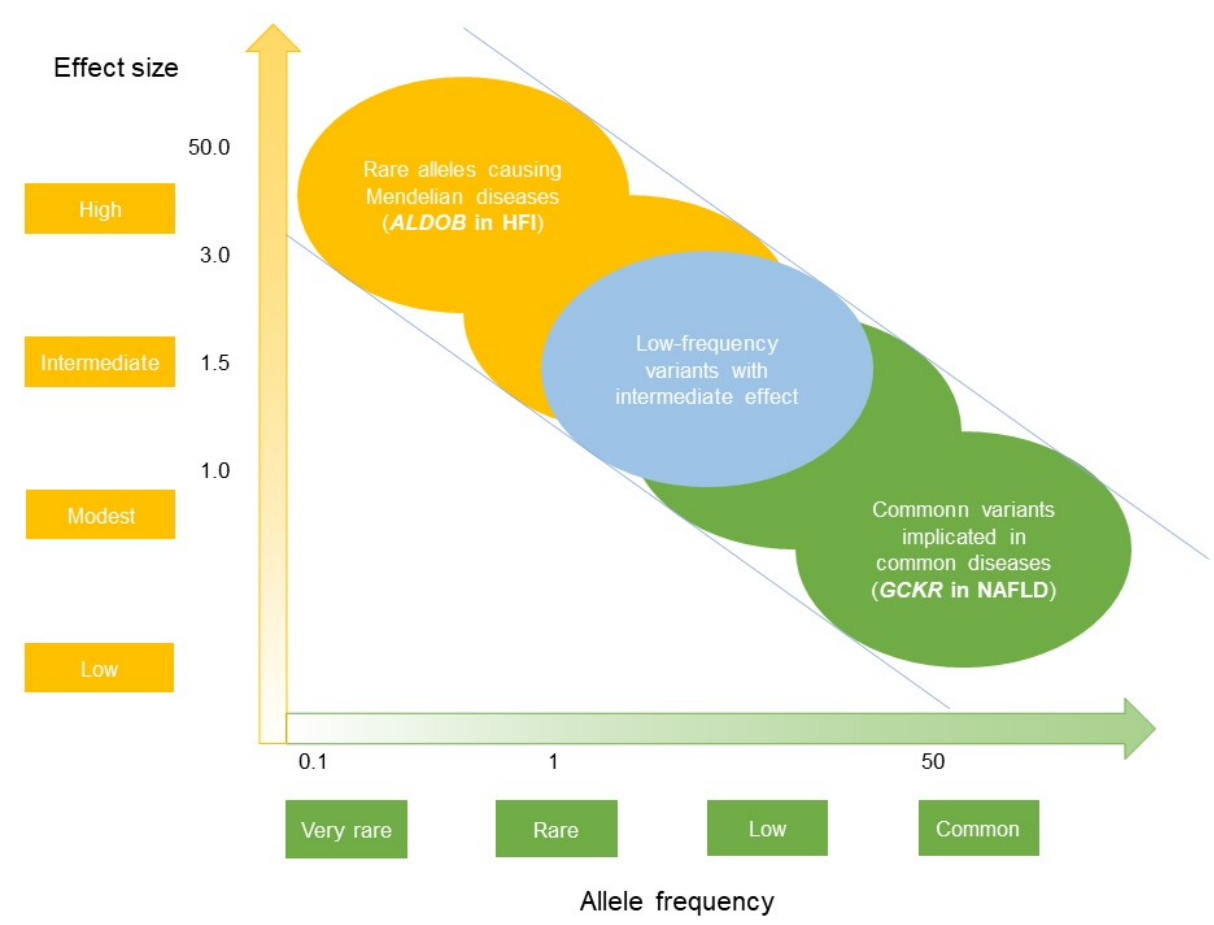

Figure 10.4 Schematic overview of the relationship between the minor allele frequency and its effect size. Schematic overview of the relationship between the minor allele frequency and its effect size. Hereditary fructose intolerance is an example of a disease caused by a rare mutation with a large effect size. On the other side of the spectrum the contribution of common genetic variants in GCKR in NAFLD, for example, is limited by their small effect sizes. Figure adapted from Manolio (Nature 2010). Abbreviations: aldolase $B$ gene $(A L D O B)$, hereditary fructose intolerance (HFI), glucokinase regulatory protein gene (GCKR), nonalcoholic fatty liver disease (NAFLD).

\subsection{Use of models and gold standard methods alternatives}

The gold standard to assess metabolic pathways is by labeling molecules with stable isotopes to create tracers. ${ }^{74}$ As studies using stable isotopes are invasive and expensive, its use in large study populations is limited. Yet, in order to assess the relationship between the different biochemical pathways leading to NAFLD and CVD (for which high study numbers are required), we used a second-best method. As discussed in detail in paragraph 2.1.1, Mendelian randomization has some disadvantages (e.g., risk of horizontal pleiotropy). However, it also offers the opportunity to make causal inferences based on observational data. For our objective, rs1260326 (GCKR) was particularly suitable, as it has a high allele frequency $(\sim 34 \%)^{10}$, is liver- 
specific $^{75}$, is proven to be a functional variant ${ }^{9}$, and has been associated with NAFLD ${ }^{20}$ as well as de novo lipogenesis. ${ }^{29}$

In part III of this thesis we aimed to study the cardiometabolic effects of enhanced dissociation of glucokinase from glucokinase regulatory protein (and, consequently, increased de novo lipogenesis) in humans. We were, however, not able to directly measure hepatic glucokinase-glucokinase regulatory protein dissociation as this would require liver biopsy, which has several major limitations (mainly poor accessibility and risk of (life-threatening) complications). Instead, we used a different approach and studied the effects of fructose. Fructose-1-phospate is known to disrupt the binding between glucokinase and glucokinase regulatory protein. ${ }^{75}$ By measuring the surrogate marker hypoglycosylated transferrin in patients with HFI (chapter seven), we were able to estimate hepatic fructose-1-phosphate concentrations. This is supported by an experimental study, in which aldolase $\mathrm{B}^{-/-}$mice chronically exposed to low amounts of fructose (similar to HFI) were also characterized by elevated hepatic fructose-1-phosphate concentrations and increased cytosolic expression of glucokinase. ${ }^{53}$ However, it could be debated whether patients with $\mathrm{HFI}$ are a good model to study glucokinase-glucokinase regulatory protein dissociation in the liver. As mentioned previously, mutations, as is the case in HFI, generally have large effect sizes ${ }^{72}$ and could, similar to experimental animal studies, not directly be extrapolated to the 'normal' general population. Extreme genetic defects for example could also affect other unknown genes and pathways (=horizontal pleiotropy), thereby confounding the results.

\subsection{Accuracy and adherence to interventions in randomized controlled trials}

In part III of this thesis we performed a double-blind randomized controlled trial with an isocaloric comparator to assess the effect of fructose restriction on $\mathrm{IHL}$ accumulation. The quality of data from randomized controlled trials relies for a large part on the compliance to the interventions that are imposed to the participants, which is a major challenge in nutrition intervention trials. The use of a control group with a similar diet and isocaloric comparator - like we have done - is therefore of great importance. In addition, we have objectively measured adherence via urinary fructose concentration, which is in contrast to the majority of the nutritional trials that mostly only use 24-hour dietary recall and/or food frequency questionnaires. Previous studies have shown that urinary fructose concentration is a good marker of fructose intake. ${ }^{76}$ Although we confirmed that the participants in the intervention group consumed less fructose than controls, it remains uncertain whether they really restricted 
fructose intake below the prescribed 7.5 and 10 grams per meal and day, respectively. Experimental studies have shown that ingestion of already 7.5 grams fructose dissociates glucokinase from glucokinase regulatory protein. ${ }^{52,77}$ However, the actual threshold by which fructose causes this dissociation (probably below 7.5 grams) is unknown. Since we were not able to measure dissociation of glucokinase from glucokinase regulatory protein in or ex vivo (for reasons described above), we can, therefore, not exclude that the observed difference in IHL content is accounted for by other mechanisms than the glucokinase-glucokinase regulatory protein complex.

\section{Clinical applications}

The key findings of this thesis could have consequences for daily clinical practice. NAFLD is generally an asymptomatic disease with low clinical awareness. Yet, the prevalence and associated morbidity and mortality of this multifactorial disease are rising, which calls for a large-scale integrated treatment approach. The results of this thesis have implications for risk stratification in patients with NAFLD and potential new drugs targeting NAFLD and CVD.

\subsection{Risk stratification}

Although NAFLD is a known cardiovascular risk factor and the main cause of death in individuals with NAFLD is CVD, this thesis has shown that NAFLD per se does not cause CVD. In addition, we have demonstrated that the cardiovascular risk depends on the pathway leading to $\mathrm{IHL}$ accumulation, in which pathways resulting in increased secretion of triglyceride-rich VLDL particles (and hence a more atherogenic lipid profile) - such as de novo lipogenesis - are especially deleterious for cardiovascular health. Practically this means that cardiovascular risk management (i.e., early diagnosis, risk assessment and optimal treatment) should be tailored to each individual.

Measurement of plasma lipids could be decisive to start primary cardiovascular prevention strategies in individuals with NAFLD, since we have shown that the increased risk of developing CVD is mainly attributed to plasma lipid levels. As the effect sizes of rs1260326 (GCKR), rs738409 (PNPLA3) and rs58542926 (TM6SF2) are relatively small, routine genotyping of these NAFLD susceptibility genes to discriminate between individuals with an increased or decreased cardiovascular risk is not recommended. 
In the search of new NAFLD biomarkers, sE-selectin could potentially be used as a marker of NAFLD severity. Indeed, as the current gold standard (i.e., liver histology) is invasive, there is a high need for feasible prognostication and disease monitoring. We found a clear association between $\mathrm{sE}$-selectin levels and NAFLD severity. However, the modest area under the ROC curve for sEselectin to differentiate between NASH and non-NASH (area under the receiver operating characteristic curve $=0.73$, chapter six), makes sE-selectin unsuitable as a single biomarker. Instead, it is anticipated that the addition of sE-selectin to a multiple biomarker panel (e.g., in combination with cytokeratin18 fragment levels ${ }^{78}$ ) will lead to a tool to non-invasively diagnose NASH in clinical practice.

\subsection{Potential new drugs targeting NAFLD and CVD}

The current NAFLD epidemic has led to the development of new drugs that now enter phase II and III clinical trials. ${ }^{79}$ Based on the outcomes presented in this thesis, we believe that future pharmacological interventions targeting NAFLD should always include plasma lipids as an outcome measure, as secondary dyslipidemia (and hence development of CVD) would seriously limit the application of these newly developed drugs.

Recently, small molecules that disrupt the binding between glucokinase and glucokinase regulatory protein have been proposed as a potential new class of antidiabetic drugs. These small molecule disruptors have indeed been shown to affectively lower plasma glucose levels in mice. ${ }^{80}$ This thesis, however, strongly suggests that increased glucokinase-glucokinase regulatory protein dissociation will have disadvantageous side-effects, such as IHL accumulation and subsequent dyslipidemia and CVD. Glucokinase-glucokinase regulatory protein enhancement, on the other hand, could be an alternative pharmacological target for NAFLD treatment. The occurrence of hyperglycemia and possible deterioration of GFR (chapter three) may limit the application of such enhancers, although both adverse side-effects may be overcome by cotreatment with sodium-glucose co-transporter-2 inhibitors, which have been shown to reduce serum glucose levels and to protect from deterioration of kidney function. ${ }^{81}$

The results from this thesis show that strict restriction of fructose is not a suitable dietary option for NAFLD patients. Adherence to a complete fructoserestricted diet is challenging and requires discipline and perseverance. Additionally, restriction of fructose intake not only includes abstinence from sugar-sweetened beverages, but also fruit and vegetables, which are 
considered healthy products. Moreover, given the small effect on IHL content, the clinical benefit of a fructose-restricted diet in the treatment of NAFLD is limited.

\section{Conclusions and future research}

In this thesis, we have shown that NAFLD per se does not cause CVD. The association between NAFLD and CVD depends on the pathway leading to IHL accumulation, in which pathways resulting in increased secretion of triglyceride-rich VLDL particles (and hence a more atherogenic lipid profile) such as de novo lipogenesis - are deleterious for cardiovascular health. In addition, we have demonstrated that E-selectin might be involved in the pathophysiology of NAFLD and that plasma sE-selectin (a biomarker that is often used to assess cardiovascular risk in epidemiological studies) might originate from the steatotic/inflamed liver. Last, we have revealed that fructose (specifically an intermediate of fructose metabolism, i.e., fructose-1-phosphate) contributes to IHL accumulation, which suggests a possible role of glucokinase regulatory protein and increased de novo lipogenesis as underlying mechanism. I believe that the results described in this thesis justify the development of new classes of drugs that intervene with the de novo lipogenesis pathway (e.g., glucokinase-glucokinase regulatory protein enhancement) for the treatment and prevention of NAFLD and CVD, respectively. As potential adverse renal side effects cannot be excluded, kidney function should be part of the scope of investigation. 


\section{Literature}

1. McAloon CJ, Boylan LM, Hamborg T, et al. The changing face of cardiovascular disease 2000-2012: An analysis of the world health organisation global health estimates data. Int J Cardiol 2016;224:256-64.

2. Lu H, Liu H, Hu F, Zou L, Luo S, Sun L. Independent Association between Nonalcoholic Fatty Liver Disease and Cardiovascular Disease: A Systematic Review and Meta-Analysis. Int J Endocrinol 2013;2013:124958.

3. Musso G, Gambino R, Cassader M, Pagano G. Meta-analysis: natural history of nonalcoholic fatty liver disease (NAFLD) and diagnostic accuracy of non-invasive tests for liver disease severity. Ann Med 2011;43:617-49.

4. Targher G, Byrne CD, Lonardo A, Zoppini G, Barbui C. Non-alcoholic fatty liver disease and risk of incident cardiovascular disease: A meta-analysis. J Hepatol 2016;65:589-600.

5. Eslam M, George J. Genetic contributions to NAFLD: leveraging shared genetics to uncover systems biology. Nat Rev Gastroenterol Hepatol 2019;17(1):40-52..

6. Trepo E, Romeo S, Zucman-Rossi J, Nahon P. PNPLA3 gene in liver diseases. J Hepatol 2016;65:399-412.

7. Mahdessian H, Taxiarchis A, Popov S, et al. TM6SF2 is a regulator of liver fat metabolism influencing triglyceride secretion and hepatic lipid droplet content. Proc Natl Acad Sci U S A 2014;111:8913-8.

8. Luukkonen PK, Zhou Y, Hyotylainen T, et al. The MBOAT7 variant rs641738 alters hepatic phosphatidylinositols and increases severity of non-alcoholic fatty liver disease in humans. $J$ Hepatol 2016;65:1263-5.

9. Beer NL, Tribble ND, McCulloch LJ, et al. The P446L variant in GCKR associated with fasting plasma glucose and triglyceride levels exerts its effect through increased glucokinase activity in liver. Hum Mol Genet 2009;18:4081-8.

10. Orho-Melander M, Melander O, Guiducci $\mathrm{C}$, et al. Common missense variant in the glucokinase regulatory protein gene is associated with increased plasma triglyceride and Creactive protein but lower fasting glucose concentrations. Diabetes 2008;57:3112-21.

11. Lindholm E, Agardh E, Tuomi T, Groop L, Agardh CD. Classifying diabetes according to the new WHO clinical stages. Eur J Epidemiol 2001;17:983-9.

12. Cole CB, Nikpay M, Lau P, et al. Adiposity significantly modifies genetic risk for dyslipidemia. J Lipid Res 2014;55:2416-22.

13. Barata L, Feitosa MF, Bielak LF, et al. Insulin Resistance Exacerbates Genetic Predisposition to Nonalcoholic Fatty Liver Disease in Individuals Without Diabetes. Hepatol Commun 2019; 3:894-907.

14. Stender S, Kozlitina J, Nordestgaard BG, Tybjaerg-Hansen A, Hobbs HH, Cohen JC. Adiposity amplifies the genetic risk of fatty liver disease conferred by multiple loci. Nat Genet 2017;49:842-7.

15. Adiels M, Taskinen MR, Packard C, et al. Overproduction of large VLDL particles is driven by increased liver fat content in man. Diabetologia 2006;49:755-65.

16. Musso G, Gambino R, Tabibian JH, et al. Association of non-alcoholic fatty liver disease with chronic kidney disease: a systematic review and meta-analysis. PLoS Med 2014;11:e1001680.

17. Mantovani A, Zaza G, Byrne CD, et al. Nonalcoholic fatty liver disease increases risk of incident chronic kidney disease: A systematic review and meta-analysis. Metabolism 2018;79:64-76.

18. Sinn DH, Kang D, Jang HR, et al. Development of chronic kidney disease in patients with non-alcoholic fatty liver disease: A cohort study. J Hepatol 2017;67:1274-80.

19. Gu Y, Mao Y, Li H, et al. Long-term renal changes in the liver-specific glucokinase knockout mouse: implications for renal disease in maturity-onset diabetes of the young 2. Transl Res 2011;157:111-6. 
20. Speliotes EK, Yerges-Armstrong LM, Wu J, et al. Genome-wide association analysis identifies variants associated with nonalcoholic fatty liver disease that have distinct effects on metabolic traits. PLoS Genet 2011;7:e1001324.

21. Chambers JC, Zhang W, Sehmi J, et al. Genome-wide association study identifies loci influencing concentrations of liver enzymes in plasma. Nat Genet 2011;43:1131-8.

22. Kozian DH, Barthel A, Cousin E, et al. Glucokinase-activating GCKR polymorphisms increase plasma levels of triglycerides and free fatty acids, but do not elevate cardiovascular risk in the Ludwigshafen Risk and Cardiovascular Health Study. Horm Metab Res 2010;42:502-6.

23. Kolz M, Johnson T, Sanna S, et al. Meta-analysis of 28,141 individuals identifies common variants within five new loci that influence uric acid concentrations. PLoS Genet 2009;5:e1000504.

24. Vaxillaire M, Cavalcanti-Proenca C, Dechaume A, et al. The common P446L polymorphism in GCKR inversely modulates fasting glucose and triglyceride levels and reduces type 2 diabetes risk in the DESIR prospective general French population. Diabetes 2008;57:2253-7.

25. Dupuis J, Langenberg C, Prokopenko I, et al. New genetic loci implicated in fasting glucose homeostasis and their impact on type 2 diabetes risk. Nat Genet 2010;42:105-16.

26. Alexander M, Loomis AK, van der Lei J, et al. Non-alcoholic fatty liver disease and risk of incident acute myocardial infarction and stroke: findings from matched cohort study of 18 million European adults. BMJ 2019;367:15367.

27. Wacholder S, Hartge P, Lubin JH, Dosemeci M. Non-differential misclassification and bias towards the null: a clarification. Occup Environ Med 1995;52:557-8.

28. Younossi ZM, Koenig AB, Abdelatif D, Fazel Y, Henry L, Wymer M. Global epidemiology of nonalcoholic fatty liver disease-Meta-analytic assessment of prevalence, incidence, and outcomes. Hepatology 2016;64:73-84.

29. Santoro N, Caprio S, Pierpont B, Van Name M, Savoye M, Parks EJ. Hepatic De Novo Lipogenesis in Obese Youth Is Modulated by a Common Variant in the GCKR Gene. J Clin Endocrinol Metab 2015;100:E1125-32.

30. Mahajan A, Wessel J, Willems SM, et al. Refining the accuracy of validated target identification through coding variant fine-mapping in type 2 diabetes. Nat Genet 2018;50: $559-71$.

31. Meigs JB, Hu FB, Rifai N, Manson JE. Biomarkers of endothelial dysfunction and risk of type 2 diabetes mellitus. JAMA 2004;291:1978-86.

32. Hwang SJ, Ballantyne CM, Sharrett AR, et al. Circulating adhesion molecules VCAM-1, ICAM-1, and E-selectin in carotid atherosclerosis and incident coronary heart disease cases: the Atherosclerosis Risk In Communities (ARIC) study. Circulation 1997;96:4219-25.

33. Herder C, Baumert J, Zierer A, et al. Immunological and cardiometabolic risk factors in the prediction of type 2 diabetes and coronary events: MONICA/KORA Augsburg case-cohort study. PloS One 2011;6:e19852.

34. Bertola A, Park O, Gao B. Chronic plus binge ethanol feeding synergistically induces neutrophil infiltration and liver injury in mice: a critical role for E-selectin. Hepatology 2013;58:1814-23.

35. Hammoutene A, Rautou PE. Role of liver sinusoidal endothelial cells in non-alcoholic fatty liver disease. J Hepatol 2019;70:1278-91.

36. Pasarin M, La Mura V, Gracia-Sancho J, et al. Sinusoidal endothelial dysfunction precedes inflammation and fibrosis in a model of NAFLD. PLoS One 2012;7:e32785.

37. Francque $\mathrm{S}$, Laleman $\mathrm{W}$, Verbeke $\mathrm{L}$, et al. Increased intrahepatic resistance in severe steatosis: endothelial dysfunction, vasoconstrictor overproduction and altered microvascular architecture. Lab Invest 2012;92:1428-39.

38. Thorand B, Baumert J, Chambless L, et al. Elevated markers of endothelial dysfunction predict type 2 diabetes mellitus in middle-aged men and women from the general population. Arterioscler Thromb Vasc Biol 2006;26:398-405.

39. Mantovani A, Byrne CD, Bonora E, Targher G. Nonalcoholic Fatty Liver Disease and Risk of Incident Type 2 Diabetes: A Meta-analysis. Diabetes Care 2018;41:372-82. 
40. Pasarin M, Abraldes JG, Rodriguez-Vilarrupla A, La Mura V, Garcia-Pagan JC, Bosch J. Insulin resistance and liver microcirculation in a rat model of early NAFLD. J Hepatol 2011;55:1095-102.

41. Ali M, Rellos P, Cox TM. Hereditary fructose intolerance. J Med Genet 1998;35:353-65.

42. Chiu S, Sievenpiper JL, de Souza RJ, et al. Effect of fructose on markers of non-alcoholic fatty liver disease (NAFLD): a systematic review and meta-analysis of controlled feeding trials. Eur J Clin Nutr 2014;68:416-23.

43. Sobrecases H, Le KA, Bortolotti M, et al. Effects of short-term overfeeding with fructose, fat and fructose plus fat on plasma and hepatic lipids in healthy men. Diabetes Metab 2010;36: 244-6.

44. Le KA, Ith $M$, Kreis $R$, et al. Fructose overconsumption causes dyslipidemia and ectopic lipid deposition in healthy subjects with and without a family history of type 2 diabetes. Am J Clin Nutr 2009;89:1760-5.

45. Kechagias S, Ernersson A, Dahlqvist O, Lundberg P, Lindstrom T, Nystrom FH. Fast-foodbased hyper-alimentation can induce rapid and profound elevation of serum alanine aminotransferase in healthy subjects. Gut 2008;57:649-54.

46. Sevastianova K, Santos A, Kotronen A, et al. Effect of short-term carbohydrate overfeeding and long-term weight loss on liver fat in overweight humans. Am J Clin Nutr 2012;96:727-34.

47. Schwarz JM, Noworolski SM, Erkin-Cakmak A, et al. Effects of Dietary Fructose Restriction on Liver Fat, De Novo Lipogenesis, and Insulin Kinetics in Children With Obesity. Gastroenterology 2017;153:743-52.

48. McCarney R, Warner J, Iliffe S, van Haselen R, Griffin M, Fisher P. The Hawthorne Effect: a randomised, controlled trial. BMC Med Res Methodol 2007;7:30.

49. Gluchowski NL, Becuwe M, Walther TC, Farese RV, Jr. Lipid droplets and liver disease: from basic biology to clinical implications. Nat Rev Gastroenterol Hepatol 2017;14:343-55.

50. DiStefano JK. Fructose-mediated effects on gene expression and epigenetic mechanisms associated with NAFLD pathogenesis. Cell Mol Life Sci 2019.

51. Petersen KF, Laurent D, Yu C, Cline GW, Shulman GI. Stimulating effects of low-dose fructose on insulin-stimulated hepatic glycogen synthesis in humans. Diabetes 2001;50: 1263-8.

52. Shiota $\mathrm{M}$, Moore $\mathrm{MC}$, Galassetti $\mathrm{P}$, et al. Inclusion of low amounts of fructose with an intraduodenal glucose load markedly reduces postprandial hyperglycemia and hyperinsulinemia in the conscious dog. Diabetes 2002;51:469-78.

53. Lanaspa MA, Andres-Hernando A, Orlicky DJ, et al. Ketohexokinase $C$ blockade ameliorates fructose-induced metabolic dysfunction in fructose-sensitive mice. J Clin Invest 2018;128:2226-38.

54. Levine DZ. Can rodent models of diabetic kidney disease clarify the significance of early hyperfiltration?: recognizing clinical and experimental uncertainties. Clin Sci (Lond) 2008;114:109-18.

55. Helal I, Fick-Brosnahan GM, Reed-Gitomer B, Schrier RW. Glomerular hyperfiltration: definitions, mechanisms and clinical implications. Nat Rev Nephrol 2012;8:293-300.

56. Okada R, Yasuda Y, Tsushita K, Wakai K, Hamajima N, Matsuo S. Glomerular hyperfiltration in prediabetes and prehypertension. Nephrol Dial Transplant 2012;27:1821-5.

57. SchwingshackI L, Hoffmann G. Comparison of high vs. normal/low protein diets on renal function in subjects without chronic kidney disease: a systematic review and meta-analysis. PloS One 2014;9:e97656.

58. Sun ZJ, Yang YC, Wu JS, Wang MC, Chang CJ, Lu FH. Increased risk of glomerular hyperfiltration in subjects with impaired glucose tolerance and newly diagnosed diabetes. Nephrol Dial Transplant 2016;31:1295-301.

59. Danesh J, Wheeler JG, Hirschfield GM, et al. C-reactive protein and other circulating markers of inflammation in the prediction of coronary heart disease. N Engl J Med 2004;350:1387-97. 
60. Everett BM, Kurth T, Buring JE, Ridker PM. The relative strength of C-reactive protein and lipid levels as determinants of ischemic stroke compared with coronary heart disease in women. J Am Coll Cardiol 2006;48:2235-42.

61. Sattar N, Murray HM, McConnachie A, et al. C-reactive protein and prediction of coronary heart disease and global vascular events in the Prospective Study of Pravastatin in the Elderly at Risk (PROSPER). Circulation 2007;115:981-9.

62. Zacho J, Tybjaerg-Hansen A, Jensen JS, Grande P, Sillesen H, Nordestgaard BG. Genetically elevated C-reactive protein and ischemic vascular disease. N Engl J Med 2008;359:1897-908.

63. Holmes MV, Ala-Korpela M, Smith GD. Mendelian randomization in cardiometabolic disease: challenges in evaluating causality. Nat Rev Cardiol 2017;14:577-90.

64. Burgess S, Foley CN, Zuber V. Inferring Causal Relationships Between Risk Factors and Outcomes from Genome-Wide Association Study Data. Annu Rev Genomics Hum Genet 2018;19:303-27.

65. Donnelly KL, Smith Cl, Schwarzenberg SJ, Jessurun J, Boldt MD, Parks EJ. Sources of fatty acids stored in liver and secreted via lipoproteins in patients with nonalcoholic fatty liver disease. J Clin Invest 2005;115:1343-51.

66. Brouwers MC, Jacobs C, Bast A, Stehouwer CD, Schaper NC. Modulation of Glucokinase Regulatory Protein: A Double-Edged Sword? Trends Mol Med 2015;21:583-94.

67. DeFilippis AP, Blaha MJ, Martin SS, et al. Nonalcoholic fatty liver disease and serum lipoproteins: the Multi-Ethnic Study of Atherosclerosis. Atherosclerosis 2013;227:429-36.

68. Simons P, Simons N, Stehouwer CDA, Schalkwijk CG, Schaper NC, Brouwers M. Association of common gene variants in glucokinase regulatory protein with cardiorenal disease: A systematic review and meta-analysis. PloS One 2018;13:e0206174.

69. Dongiovanni $P$, Stender S, Pietrelli A, et al. Causal relationship of hepatic fat with liver damage and insulin resistance in nonalcoholic fatty liver. J Intern Med 2018;283:356-70.

70. Luukkonen PK, Sadevirta S, Zhou Y, et al. Saturated Fat Is More Metabolically Harmful for the Human Liver Than Unsaturated Fat or Simple Sugars. Diabetes Care 2018;41:1732-9.

71. Markova M, Pivovarova O, Hornemann S, et al. Isocaloric Diets High in Animal or Plant Protein Reduce Liver Fat and Inflammation in Individuals With Type 2 Diabetes. Gastroenterology 2017;152:571-85.e8.

72. Manolio TA, Collins FS, Cox NJ, et al. Finding the missing heritability of complex diseases. Nature 2009;461:747-53.

73. Simons N, Isaacs A, Koek GH, Kuc S, Schaper NC, Brouwers MC. PNPLA3, TM6SF2, and MBOAT7 Genotypes and Coronary Artery Disease. Gastroenterology 2017;152:912-3.

74. Umpleby AM. HORMONE MEASUREMENT GUIDELINES: Tracing lipid metabolism: the value of stable isotopes. $J$ Endocrinol 2015;226:G1-10.

75. van Schaftingen $E$, Vandercammen $A$, Detheux $M$, Davies $D R$. The regulatory protein of liver glucokinase. Adv Enzyme Regul 1992;32:133-48.

76. Tasevska N, Runswick SA, McTaggart A, Bingham SA. Urinary sucrose and fructose as biomarkers for sugar consumption. Cancer Epidemiol Biomarkers Prev 2005;14:1287-94.

77. Shiota M, Galassetti P, Monohan M, Neal DW, Cherrington AD. Small amounts of fructose markedly augment net hepatic glucose uptake in the conscious dog. Diabetes 1998;47: 867-73.

78. Wieckowska A, Zein NN, Yerian LM, Lopez AR, McCullough AJ, Feldstein AE. In vivo assessment of liver cell apoptosis as a novel biomarker of disease severity in nonalcoholic fatty liver disease. Hepatology 2006;44:27-33.

79. Francque S, Vonghia L. Pharmacological Treatment for Non-alcoholic Fatty Liver Disease. Adv Ther 2019;36:1052-74.

80. Lloyd DJ, St Jean DJ, Jr., Kurzeja RJ, et al. Antidiabetic effects of glucokinase regulatory protein small-molecule disruptors. Nature 2013;504:437-40.

81. Perkovic V, Jardine MJ, Neal B, et al. Canagliflozin and Renal Outcomes in Type 2 Diabetes and Nephropathy. N Engl J Med 2019;380:2295-306. 
-

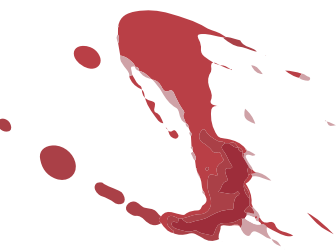

-

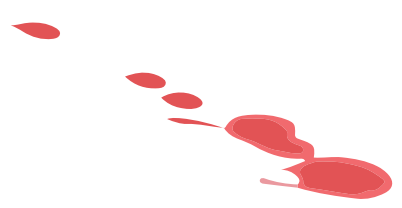

Nederlandse samenvatting
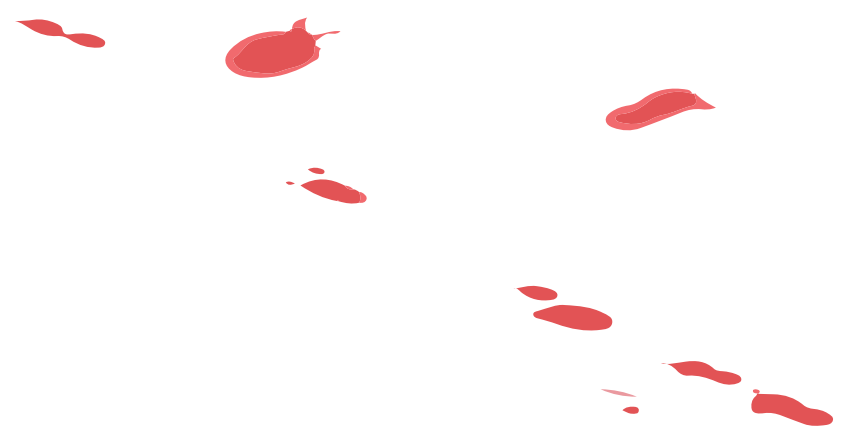

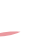




\section{Introductie}

Hart- en vaatziekten zijn één van de voornaamste doodsoorzaken in de wereld. Oudere leeftijd, mannelijk geslacht, hypertensie (hoge bloeddruk), hyperlipidemie (hoog cholesterol- en vetgehalte), diabetes mellitus (suikerziekte), obesitas en roken zijn de belangrijkste risicofactoren voor het ontwikkelen van hart- en vaatziekten. Onlangs is aangetoond dat ook individuen met niet-alcoholische leververvetting een verhoogd risico hebben op het ontwikkelen van hart- en vaatziekten. Niet-alcoholische leververvetting komt voor bij één op de vier volwassenen en is een aandoening die bestaat uit steatose (vervetting), inflammatie (ontsteking) en/of fibrosering (verlittekening) van de lever in afwezigheid van overmatig alcoholgebruik.

Er zijn verschillende mechanismen die leiden tot niet-alcoholische leververvetting. Ten eerste kan een overmaat aan vet in de bloedbaan (dat afkomstig is van voedsel of vetcellen) in de lever opgeslagen worden. Er is dan sprake van een verhoogde flux van vrije vetzuren richting de lever. Ten tweede kan een overmaat aan glucose (suiker) uit de bloedbaan in de lever omgezet en opgeslagen worden als vet. Dit wordt verhoogde de novo lipogenese genoemd. Ten derde kan het opgeslagen vet in de lever onvoldoende verbrand/gebruikt worden als energiebron. Er wordt dan gesproken over verlaagde beta-oxidatie. Ten slotte kan het opgeslagen vet in de lever onvoldoende getransporteerd worden naar de bloedbaan om elders in het lichaam gebruikt te worden. $\mathrm{Er}$ is dan sprake van een verlaagde productie en secretie van VLDL-partikels.

Op dit moment is het nog niet duidelijk of niet-alcoholische leververvetting een onschuldige bijstander of een actieve bijdrager is aan de ontwikkeling van harten vaatziekten. In geval van het laatste worden verschillende mechanismen verondersteld, waaronder hyperlipidemie, laaggradige inflammatie, hypercoagulatie/verminderde fibrinolyse (verhoogde stollingsneiging), hepatokines (eiwitten afkomstig uit de lever die metabole processen in het lichaam beïnvloeden) en insuline resistentie/hyperglycemie (ongevoeligheid voor de werking van insuline/verhoogd plasma glucosewaarden in het bloed).

Het doel van dit proefschrift was te onderzoeken of niet-alcoholische leververvetting oorzakelijk verbonden is met hart- en vaatziekten en of deze oorzakelijke relatie afhangt van het mechanisme dat ten grondslag ligt aan (dat leidt tot) niet-alcoholische leververvetting. 


\section{Belangrijkste bevindingen}

\subsection{Genetische predispositie voor niet-alcoholische leververvetting en het risico op hart- en vaatziekten}

In het eerste deel van dit proefschrift hebben wij gebruik gemaakt van meerdere genetische-epidemiologische studies om de oorzakelijke relatie te onderzoeken tussen niet-alcoholische leververvetting en hart- en vaatziekten. Hiervoor hebben wij gebruikt gemaakt van SNP's (single nucleotide polymorphisms/enkel-nucleotide polymorfismes) als modellen voor de verschillende mechanismen die ten grondslag liggen aan niet-alcoholische leververvetting. SNP's zijn veel voorkomende variaties in de DNA-volgorde (specifiek in een enkele nucleotide), die soms het functioneren van genen beïnvloeden. Zo is uit eerder onderzoek gebleken dat bepaalde SNP's leiden tot niet-alcoholische leververvetting. De SNP's rs738409 (PNPLA3) en rs58542926 (TM6SF2) bijvoorbeeld leiden tot niet-alcoholische leververvetting via verminderde productie en secretie van VLDL-partikels. De SNP rs1260326 (GCKR) daarentegen leidt tot niet-alcoholische leververvetting via verhoogde de novo lipogenese.

In hoofdstuk 3 hebben wij gevonden dat individuen met de PNPLA3 en TM6SF2 SNP's minder vaak hart- en vaatziekten ontwikkelen dan individuen zonder deze genetische variaties. In hoofdstuk 4 hebben wij laten zien dat individuen met de GCKR SNP vaker hart- en vaatziekten ontwikkelen in vergelijking met individuen zonder deze genetische variatie. Daarnaast hebben individuen met de GCKR SNP een hogere glomerulaire filtratiesnelheid (een maat voor nierfunctie) en zijn mogelijk beschermd tegen chronische nierziekten (een belangrijke risicofactor voor het ontwikkelen van hart- en vaatziekten). In hoofdstuk 2 hebben wij aangetoond dat individuen met de GCKR SNP én diabetes mellitus type 2 (suikerziekte) een slechter cardiovasculair lipidenprofiel hebben (specifiek meer triglyceriden/vetten en minder HDLl'goede' cholesterol in het bloed) in vergelijking met individuen zonder deze genetische variatie. In hoofdstuk 5 hebben wij zichtbaar gemaakt dat er een grote heterogeniteit (diversiteit) bestaat binnen de verschillende SNP's die leiden tot niet-alcoholische leververvetting en het ontwikkelen van hart- en vaatziekten. In aanvulling hebben wij aangetoond dat alléén individuen met SNP's die leiden tot een slechter cardiovasculair lipidenprofiel (zoals GCKR) vaker hart- en vaatziekten ontwikkelen. 


\subsection{De relatie tussen niet-alcoholische leververvetting en sE- selectine, een marker voor hart- en vaatziekten}

Voortbordurend op de resultaten van het eerste deel van dit proefschrift, hebben wij in het tweede deel de relatie onderzocht tussen de SNP's rs738409 (PNPLA3) en rs1260326 (GCKR) en indirecte markers van hart- en vaatziekten. Soluble endothelium selectin (sE-selectine) en soluble vascular cell adhesion molecule 1 (sVCAM) zijn twee biomarkers die binnen wetenschappelijk onderzoek regelmatig gebruikt worden om het cardiovasculaire risico van een populatie in te schatten. Hierbij geldt dat hoe hoger de sE-selectin- en sVCAM-waarden in het bloed, des te hoger het risico om hart- en vaatziekten te ontwikkelen.

In tegenstelling tot onze verwachting hebben wij in hoofdstuk 6 gevonden dat zowel individuen met de PNPLA3 als de GCKR SNP in het bloed verhoogde $s E-s e l e c t i n e-w a a r d e n ~ h e b b e n$ (indicatief voor een verhoogd cardiovasculair risico). Dit resultaat is in tegenstrijd met het eerste deel van het proefschrift, waarin wij hebben laten zien dat individuen met de PNPLA3 SNP minder vaak hart- en vaatziekten ontwikkelen. Wij hebben de relatie tussen $\mathrm{sE}$-selectine en niet-alcoholische leververvetting vervolgens verder onderzocht in een experimentele studie met muizen en mensen met ernstige obesitas en aangetoond dat er een relatie bestaat tussen de sE-selectine waarden in het bloed, de ernst/mate van niet-alcoholische leververvetting en de expressie (tot uiting komen) van het E-selectine-gen in de lever. Ook bestaat er een relatie tussen ALAT (een marker voor leverschade/niet-alcoholische leververvetting) en sE-selectine, onafhankelijk van andere factoren. Tezamen suggereren deze resultaten dat E-selectine betrokken is bij de pathofysiologie (ontstaanswijze) van niet-alcoholische leververvetting en dat sE-selectine in het bloed afkomstig is van de vervette/ontstoken lever.

\subsection{De rol van glucokinase regulatory protein in het ontstaan van niet-alcoholische leververvetting}

In het derde deel van dit proefschrift hebben wij de relatie onderzocht tussen niet-alcoholische leververvetting en glucokinase regulatory protein (het eiwit dat gecodeerd wordt door het GCKR gen). Wij hebben dit onderzocht middels de monosacharide fructose (vruchtensuiker). Fructose wordt primair in de lever omgezet in onder andere vet. Een bijproduct van deze omzetting (fructose-1fosfaat) is tevens instaat het eiwit glucokinase te activeren door middel van het ontkoppelen van glucokinase van zijn regulerend eiwit, genaamd glucokinase regulatory protein. Als gevolg hiervan neemt de opname van glucose door de lever toe en daarmee ook de vorming van vet (via de novo lipogenese). 
Wij hebben de hoeveelheid levervet en cardiovasculaire risicofactoren onderzocht in patiënten met hereditaire fructose intolerantie (een stofwisselingsziekte gekenmerkt door een verminderde afbraak van fructose-1fosfaat) en in proefpersonen met overgewicht die gedurende zes weken een fructose-beperkt dieet hebben gevolgd. In hoofdstuk 7 hebben wij laten zien dat patiënten met hereditaire fructose intolerantie meer levervet hebben dan individuen met hetzelfde geslacht en dezelfde leeftijd en body mass index. Wij hebben aanwijzingen gevonden dat dit verschil veroorzaakt wordt door een verhoogde hoeveelheid fructose-1-fosfaat in de lever. Deze patiënten hebben eveneens een hoger risico op het ontwikkelen van suikerziekte. In hoofdstuk 9 hebben wij aangetoond dat patiënten met hereditaire fructose intolerantie hogere bloeddrukken en hogere sE-selectine-waarden in het bloed hebben. In lijn met hoofdstuk 4 hebben deze patiënten eveneens een hogere glomerulaire filtratiesnelheid (een maat voor nierfunctie). In hoofdstuk 8 hebben wij gevonden dat een dieet arm in fructose gedurende zes weken leidt tot een afname van de hoeveelheid levervet, maar niet tot een afname in het risico op het ontwikkelen van suikerziekte of een afname in het risico op hart- en vaatziekten (gemeten aan de hand van het lipidenprofiel).

\section{Conclusie}

Op basis van dit proefschrift concluderen wij dat niet-alcoholische leververvetting per se géén hart- en vaatziekten veroorzaakt. De relatie tussen niet-alcoholische leververvetting en hart- en vaatziekten hangt namelijk af van het mechanisme dat ten grondslag ligt aan niet-alcoholische leververvetting, waarbij verhoogde productie en secretie van VLDL-partikels - bijvoorbeeld via de novo lipogenese - vooral schadelijk is voor de cardiovasculaire gezondheid. Tevens concluderen wij dat fructose (specifiek een tussenproduct van de omzetting van fructose, namelijk fructose-1-fosfaat) bijdraagt aan het ontwikkelen van leververvetting, met het eiwit glucokinase regulatory protein en de novo lipogenese als mogelijk onderliggend mechanisme. 
-

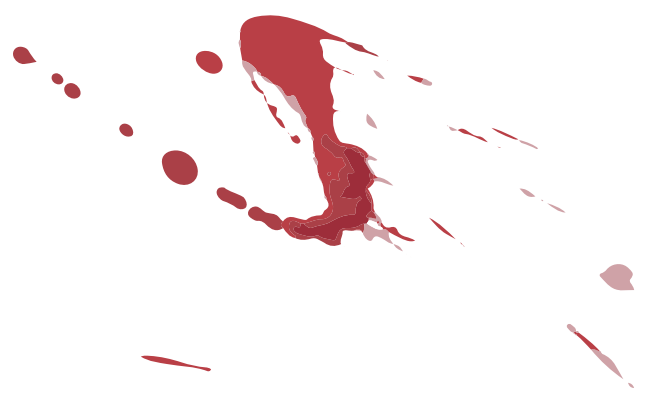

Valorisation addendum 


\section{Introduction}

Cardiovascular disease (CVD) is a major threat to global health. ${ }^{1}$ In 2017, approximately 17.8 million people died as a consequence of CVD. ${ }^{2}$ Several risk factors for CVD have been identified, including - but not limited to - age, male sex, smoking, high blood pressure, high cholesterol level, obesity and diabetes mellitus. ${ }^{3,4}$

Recently, another risk factor has been added to the list; nonalcoholic fatty liver disease (NAFLD) is a histological spectrum comprising hepatic steatosis (presence of fat in the liver), steatohepatitis (presence of fat and inflammation in the liver) and fibrosis ${ }^{5}$, in the absence of excessive alcohol intake. It occurs in $25 \%$ of the adult population ${ }^{6}$ and the prevalence of NAFLD is even higher in specific populations, such as type 2 diabetes mellitus. ${ }^{7}$ The first stage of NAFLD (i.e., hepatic steatosis) results from an imbalance between the formation and degradation of fat in the liver, either due to (1) increased flux of free fatty acids towards the liver (the backbones of fat), (2) increased de novo lipogenesis (the formation of fat from glucose), (3) impaired beta-oxidation (the breakdown of fatty acids as energy source) or (4) impaired production and secretion of VLDL particles (the transporters of fat through the body).

Already much research has been performed on the role of NAFLD in the development of CVD. However, it remains unclear whether NAFLD is an active contributor to cardiovascular risk or just an innocent bystander. This distinction is essential as it requires a different prevention and treatment approach. In the current thesis, we have tried to gain insight into the causal relationship between NAFLD and CVD, and possible underlying mechanisms.

This chapter describes the value of the performed research for society in general and for clinicians and researchers in specific.

\section{2. (Cardiovascular) risk management in NAFLD patients}

In spite of the increasing prevalence and associated morbidity and mortality, NAFLD is an asymptomatic disease with low awareness among patients and clinicians. In order to adequately treat patients, a good understanding of disease etiology is essential. This thesis has gained insight into the causal relationship between NAFLD and CVD. We have shown that NAFLD per se does not cause CVD and that the cardiovascular risk in NAFLD patients 
depends on the pathway that leads to intrahepatic lipid accumulation (IHL) accumulation. Specifically, pathways that result in increased secretion of triglyceride-rich VLDL particles (and hence a more atherogenic lipid profile) - such as de novo lipogenesis - are deleterious for cardiovascular health. This finding has relevant consequences for the daily clinical practice. Since not all NAFLD patients carry the same cardiovascular risk, disease management should be tailored to each individual patient. Measurement of plasma lipids (and assessment of the overall cardiovascular risk profile) could be leading in the decision to start cardiovascular treatment in individuals with NAFLD, as we have shown that the increased risk of developing CVD is mainly attributed to plasma lipid levels. The current guidelines recommending screening for dyslipidemia are in line with the results of this thesis. ${ }^{5}$ Aside from standard treatment with HMG-CoA-Reductase Inhibitors (statins), pharmacological options that have proven to affect both NAFLD and CVD, such as pioglitazone and liraglutide ${ }^{8-11}$, could be considered (although the former recommendation is hampered by other side-effects, such as heart failure and fractures ${ }^{12}$, and the latter recommendation first needs to be supported by more large-scale randomized controlled trials).

In addition to prevention of CVD in NAFLD patients, there is an ongoing search for noninvasive biomarkers for feasible prognostication and disease monitoring of NAFLD itself, as it could progress into cirrhosis and hepatocellular carcinoma. ${ }^{13}$ Moreover, NAFLD is expected to become the primary indication for liver transplantation by $2025 .{ }^{14}$ However, the current gold standard for NAFLD, in particular NASH (i.e., liver histology), is invasive and poorly tolerated. This thesis has provided insight into a potential new biomarker for NAFLD severity; plasma soluble E-selectin. We showed that: 1) NAFLD severity, in particular the inflammatory stage, was associated with hepatic Eselectin expression; 2) E-selectin mRNA expression in liver, but not in other organ tissue, was associated with plasma sE-selectin levels; and 3) NAFLD susceptibility genes and liver parenchyma damage (reflected by plasma alanine aminotransferase levels levels) were associated with plasma sEselectin levels, independent of potential confounding factors. These results, however, need to be replicated in cohort studies with biopsy proven NAFLD before $\mathrm{SE}$-selectin could be considered as part of the biomarker panel for NAFLD. 


\section{Fructose-1-phosphate and glucokinase regulatory protein as potential treatment targets}

The phenotypic manifestation and severity of NAFLD is the result of multiple gene-environment interactions. ${ }^{15}$ Fructose has been suggested to play a key role in the development of NAFLD as it is primarily metabolized in the liver. ${ }^{16}$ Yet, trials on the effect of fructose on IHL content in humans show conflicting results. ${ }^{17}$ This thesis has gained insight into the role of fructose in the development of NAFLD. We have found indications that specifically fructose-1phosphate (an intermediate of fructose metabolism) is an important factor in $\mathrm{IHL}$ accumulation (via de novo lipogenesis). This insight provides a new starting point for future research, more specific on molecules that block the formation of fructose-1-phosphate, for example via inhibition of ketohexokinase (the enzyme responsible for the conversion of fructose to fructose-1phosphate). Indeed, by reducing the intrahepatic fructose-1-phosphate concentration, both the direct lipogenic effects (i.e., increased de novo lipogenesis) and indirect lipogenic effects (i.e., increased disruption of the glucokinase-glucokinase regulatory protein complex) of fructose could be prevented. The subsequent elevated levels of plasma fructose (which also occurs in patients with a ketohexokinase deficiency known as essential fructosuria (OMIM\# 229800)) is clinically asymptomatic and harmless, which further emphasize ketohexokinase as potential new therapeutic target. This new type of drug would not only reduce IHL accumulation in NAFLD patients, but could also be of benefit for HFI patients that are currently still bound to a lifelong diet that is devoid of fructose. Besides emphasizing the need for additional treatment, this thesis also provided new information on the long-term consequences of $\mathrm{HFI}$ for practice guidelines, which up to now were primary based on case reports (of children) at time of diagnosis.

An alternative target for NAFLD treatment could be the glucokinaseglucokinase regulatory protein complex. Recently, small molecules that disrupt the binding between glucokinase and glucokinase regulatory protein have been proposed as a potential new class of antidiabetic drugs. These small molecule disruptors have indeed been shown to affectively lower plasma glucose levels in mice. ${ }^{18}$ This thesis, however, strongly suggests that increased glucokinaseglucokinase regulatory protein dissociation will have disadvantageous sideeffects, such as IHL accumulation and subsequent dyslipidemia and CVD development. Glucokinase-glucokinase regulatory protein enhancement, on the other hand, could be an alternative pharmacological target for NAFLD treatment. The occurrence of hyperglycemia as potential negative side effect 
would, of course, require additional blood glucose lowering medication (for example with the use of sodium-glucose co-transporter-2 inhibitors).

For both potential new treatment targets, mouse models are available. ${ }^{19,20}$ The results of this thesis have directly led to the set-up of new studies, aimed at reducing the $\mathrm{IHL}$ content by interfering with either hepatic fructose metabolism or the glucokinase-glucokinase regulatory protein complex, of which the results will hopefully follow shortly.

\section{The toxic truth about fructose}

Last, this thesis has gained insight into the metabolic consequences of fructose in comparison to glucose. In the late 1960s, fructose was introduced to the food industry as a sweeter alternative for glucose. Since then, it has been widely distributed and used in sugar-sweetened beverages and processed foods. Since the 1980s, however, there have been doubts about the negative health consequences of this monosaccharide as the rise in fructose intake paralleled the increase in obesity and its related metabolic complications, such as NAFLD. ${ }^{21-23}$ For a long time, fructose has been the scope of research and although there is abundant evidence from animal studies on the prominent role and putative mechanism by which fructose causes $\mathrm{IHL}$ accumulation ${ }^{24-28}$, a previous meta-analysis of controlled trials in humans did not show any effects of fructose on IHL content. ${ }^{17}$ The included studies, however, often used a fructose overdose in combination with a hypercaloric diet. ${ }^{29-32}$ We are the first that studied the effects of fructose restriction in overweight adults in a randomized controlled setting and found that removal of fructose from the diet does have additional health benefits (i.e., reducing $\mathrm{IHL}$ content). Thus, in a certain way one could argue if in the current Western society the phrase 'an apple a day keeps the doctor away' is outdated.

\section{Conclusion}

In conclusion, the results reported in this thesis may benefit society in general and clinicians and researchers in specific by providing new information on 1) the relationship between NAFLD, plasma lipids and CVD, which can be used in NAFLD practice guidelines; 2) a potential new biomarker for NAFLD, which in time can potentially be used for disease prognostication and monitoring; 3) potential new treatment targets for NAFLD and HFI, which in time can delay 
disease progression and improve quality of life; 4) the health benefits of fructose restriction, which can contribute to the current vision on excessive sugar intake and the need to reduce sugar processing by the industry. 


\section{Literature}

1. McAloon CJ, Boylan LM, Hamborg T, et al. The changing face of cardiovascular disease 2000-2012: An analysis of the world health organisation global health estimates data. Int J Cardiol 2016;224:256-64.

2. Global, regional, and national age-sex-specific mortality for 282 causes of death in 195 countries and territories, 1980-2017: a systematic analysis for the Global Burden of Disease Study 2017. Lancet 2018;392:1736-88.

3. Hennekens $\mathrm{CH}$. Increasing burden of cardiovascular disease: current knowledge and future directions for research on risk factors. Circulation 1998;97:1095-102.

4. Yusuf $\mathrm{S}$, Hawken $\mathrm{S}$, Ounpuu $\mathrm{S}$, et al. Effect of potentially modifiable risk factors associated with myocardial infarction in 52 countries (the INTERHEART study): case-control study. Lancet 2004;364:937-52.

5. EASL-EASD-EASO Clinical Practice Guidelines for the management of non-alcoholic fatty liver disease. J Hepatol 2016;64:1388-402.

6. Younossi ZM, Koenig AB, Abdelatif D, Fazel Y, Henry L, Wymer M. Global epidemiology of nonalcoholic fatty liver disease-Meta-analytic assessment of prevalence, incidence, and outcomes. Hepatology 2016;64:73-84.

7. Younossi ZM, Golabi P, de Avila L, et al. The global epidemiology of NAFLD and NASH in patients with type 2 diabetes: A systematic review and meta-analysis. J Hepatol 2019;71: 793-801.

8. Cusi K, Orsak B, Bril F, et al. Long-Term Pioglitazone Treatment for Patients With Nonalcoholic Steatohepatitis and Prediabetes or Type 2 Diabetes Mellitus: A Randomized Trial. Ann Intern Med 2016;165:305-15.

9. Dormandy JA, Charbonnel B, Eckland DJ, et al. Secondary prevention of macrovascular events in patients with type 2 diabetes in the PROactive Study (PROspective pioglitAzone Clinical Trial In macroVascular Events): a randomised controlled trial. Lancet 2005;366: 1279-89.

10. Armstrong MJ, Gaunt $P$, Aithal GP, et al. Liraglutide safety and efficacy in patients with nonalcoholic steatohepatitis (LEAN): a multicentre, double-blind, randomised, placebo-controlled phase 2 study. Lancet 2016;387:679-90.

11. Marso SP, Daniels GH, Brown-Frandsen K, et al. Liraglutide and Cardiovascular Outcomes in Type 2 Diabetes. N Engl J Med 2016;375:311-22.

12. Liao HW, Saver JL, Wu YL, Chen TH, Lee M, Ovbiagele B. Pioglitazone and cardiovascular outcomes in patients with insulin resistance, pre-diabetes and type 2 diabetes: a systematic review and meta-analysis. BMJ Open 2017;7:e013927.

13. Beste LA, Leipertz SL, Green PK, Dominitz JA, Ross D, loannou GN. Trends in burden of cirrhosis and hepatocellular carcinoma by underlying liver disease in US veterans, 20012013. Gastroenterology 2015;149:1471-82.e5; quiz e17-8.

14. Wong RJ, Aguilar M, Cheung R, et al. Nonalcoholic steatohepatitis is the second leading etiology of liver disease among adults awaiting liver transplantation in the United States. Gastroenterology 2015;148:547-55.

15. Eslam M, George J. Genetic and epigenetic mechanisms of NASH. Hepatol Int 2016;10: 394-406.

16. Jensen $\mathrm{T}$, Abdelmalek MF, Sullivan $\mathrm{S}$, et al. Fructose and sugar: A major mediator of nonalcoholic fatty liver disease. J Hepatol 2018;68:1063-75.

17. Chiu S, Sievenpiper JL, de Souza RJ, et al. Effect of fructose on markers of non-alcoholic fatty liver disease (NAFLD): a systematic review and meta-analysis of controlled feeding trials. Eur J Clin Nutr 2014;68:416-23. 
18. Lloyd DJ, St Jean DJ, Jr., Kurzeja RJ, et al. Antidiabetic effects of glucokinase regulatory protein small-molecule disruptors. Nature 2013;504:437-40.

19. Grimsby J, Coffey JW, Dvorozniak MT, et al. Characterization of glucokinase regulatory protein-deficient mice. J Biol Chem 2000;275:7826-31.

20. Oppelt SA, Sennott EM, Tolan DR. Aldolase-B knockout in mice phenocopies hereditary fructose intolerance in humans. Mol Genet Metab 2015;114:445-50.

21. Basciano H, Federico L, Adeli K. Fructose, insulin resistance, and metabolic dyslipidemia. Nutr Metab (Lond) 2005;2:5.

22. Nakagawa T, Tuttle KR, Short RA, Johnson RJ. Hypothesis: fructose-induced hyperuricemia as a causal mechanism for the epidemic of the metabolic syndrome. Nat Clin Pract Nephrol $2005 ; 1: 80-6$.

23. Dhingra R, Sullivan L, Jacques PF, et al. Soft drink consumption and risk of developing cardiometabolic risk factors and the metabolic syndrome in middle-aged adults in the community. Circulation 2007;116:480-8.

24. Ackerman Z, Oron-Herman M, Grozovski $M$, et al. Fructose-induced fatty liver disease: hepatic effects of blood pressure and plasma triglyceride reduction. Hypertension 2005;45:1012-8.

25. Armutcu F, Coskun O, Gürel $A$, et al. Thymosin alpha 1 attenuates lipid peroxidation and improves fructose-induced steatohepatitis in rats. Clin Biochem 2005;38:540-7.

26. Koo HY, Miyashita M, Cho BH, Nakamura MT. Replacing dietary glucose with fructose increases ChREBP activity and SREBP-1 protein in rat liver nucleus. Biochem Biophys Res Commun 2009;390:285-9.

27. Roglans $\mathrm{N}$, Vilà L, Farré $\mathrm{M}$, et al. Impairment of hepatic Stat-3 activation and reduction of PPARalpha activity in fructose-fed rats. Hepatology 2007;45:778-88.

28. Samuel VT, Liu ZX, Wang A, et al. Inhibition of protein kinase Cepsilon prevents hepatic insulin resistance in nonalcoholic fatty liver disease. J Clin Invest 2007;117:739-45.

29. Sobrecases H, Le KA, Bortolotti M, et al. Effects of short-term overfeeding with fructose, fat and fructose plus fat on plasma and hepatic lipids in healthy men. Diabetes Metab 2010;36:244-6.

30. Le KA, Ith $\mathrm{M}, \mathrm{Kreis} \mathrm{R}$, et al. Fructose overconsumption causes dyslipidemia and ectopic lipid deposition in healthy subjects with and without a family history of type 2 diabetes. Am J Clin Nutr 2009;89:1760-5.

31. Kechagias S, Ernersson A, Dahlqvist O, Lundberg P, Lindstrom T, Nystrom FH. Fast-foodbased hyper-alimentation can induce rapid and profound elevation of serum alanine aminotransferase in healthy subjects. Gut 2008;57:649-54.

32. Sevastianova K, Santos A, Kotronen A, et al. Effect of short-term carbohydrate overfeeding and long-term weight loss on liver fat in overweight humans. Am J Clin Nutr 2012;96:727-34. 
$\bullet$

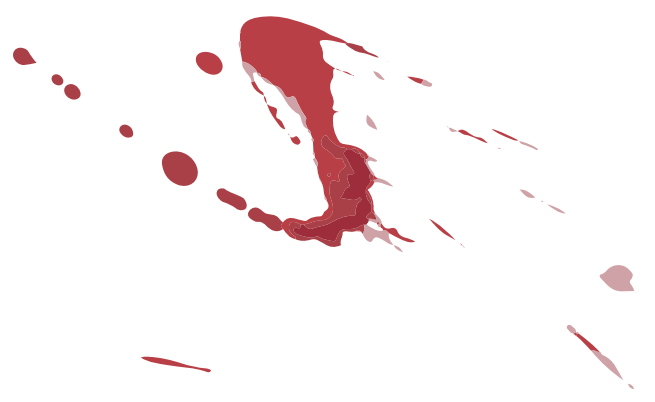

Scientific output 


\section{List of publications}

Simons N, Dekker JM, van Greevenbroek MMJ, Nijpels G, 't Hart LM, van der Kallen CJH, Schalkwijk CG, Schaper NC, Stehouwer CDA, Brouwers MCGJ. A common gene variant in glucokinase regulatory protein interacts with glucose metabolism on diabetic dyslipidemia: the combined CODAM and Hoorn studies. Diabetes Care 2016 Oct;39(10):1811-1817.

Simons N, Isaacs A, Koek GH, Kuč S, Schaper NC, Brouwers MCGJ. PNPLA3, TM6SF2 and MBOAT7 genotypes and coronary artery disease. Gastroenterology 2017 Mar;152(4):912-913.

Simons $\mathrm{PIHG}^{*}$, Simons $\mathbf{N}^{*}$, Stehouwer CDA, Schalkwijk CG, Schaper NC, Brouwers MCGJ*. Association of common gene variants in glucokinase regulatory protein with cardiorenal disease: a systematic review and metaanalysis. PLoS One 2018 Oct;13(10):e0206174.

${ }^{*}$ Equal contribution

Brouwers MCGJ, Simons N, Stehouwer CDA, Koek GH, Schaper NC, Isaacs A. Relationship between nonalcoholic fatty liver disease susceptibility genes and coronary artery disease. Hepatol Commun 2019 Feb;3(4):587-596.

Simons N, Debray FG, Schaper NC, Kooi ME, Feskens EJM, Hollak CEM, Lindeboom L, Koek GH, Bons JAP, Lefeber DJ, Hodson L, Schalkwijk CG, Stehouwer CDA, Cassiman D, Brouwers MCGJ. Patients with aldolase B deficiency are characterized by an increased intrahepatic lipid content. J Clin Endocrinol Metab 2019 Nov;104(11):5056-5064.

Brouwers MCGJ, Simons N, Stehouwer CDA, Isaacs A. Non-alcoholic fatty liver disease and cardiovascular disease: assessing the evidence for causality. Diabetologia 2020 Feb;63(2):253-260.

Brouwers MCGJ, De Graaf J, Simons N, Meex S, Ten Doeschate S, Van Heertum S, Heidemann B, Luijten J, De Boer D, Schaper NC, Stehouwer CDA, Van Greevenbroek MMJ. Incidence of type 2 diabetes in familial combined hyperlipidemia. BMJ Open Diabetes Res Care 2020 Mar;8(1):e001107.

Simons N, Bijnen $M$, Wouters KAM, Rensen SS, Beulens JWJ, van Greevenbroek MMJ, 't Hart LM, Greve JWM, van der Kallen CJH, Schaper NC, Schalkwijk CG, Stehouwer CDA, Brouwers MCGJ. The endothelial function 
biomarker soluble E-selectin is associated with nonalcoholic fatty liver disease. Liver Int 2020 May;40(5):1079-1088.

Simons N, Debray FG, Schaper NC, Feskens EJM, Hollak CEM, Bons JAP, Bierau J, Houben AJHM, Schalkwijk CG, Stehouwer CDA, Cassiman D, Brouwers MCGJ. Kidney and vascular function in adult patients with hereditary fructose intolerance. Mol Genet Metab Rep 2020 May;23:100600.

Buziau AM, Scheijen JLJM, Stehouwer CDA, Simons N, Brouwers MCGJ, Schalkwijk CG. Development and validation of a UPLC-MS/MS method to quantify fructose in serum and urine. J Chromatogr B Analyt Technol Biomed Life Sci 2020 Oct;1155:122299.

Simons N, Veeraiah P, Simons PIHG, Schaper NC, Kooi ME, SchrauwenHinderling VB, Feskens EJM, van der Ploeg EMC, Van den Eynde MDG, Schalkwijk CG, Stehouwer CDA, Brouwers MCGJ. Effects of fructose restriction on liver steatosis (FRUITLESS); a double-blind randomized controlled trial. American Journal of Clinical Nutrition 2020. Accepted.

\section{Oral presentations}

NVDO, Annual Dutch Diabetes Research Meeting 2016, Oosterbeek (The Netherlands)

Maastricht-Lille-Düsseldorf Diabetes Symposium 2017, Düsseldorf (Germany)

NVDO, Annual Dutch Diabetes Research Meeting 2017, Oosterbeek (The Netherlands)

Dutch Endocrine Meeting 2018, Noordwijkerhout (The Netherlands)

Maastricht-Lille-Düsseldorf Diabetes Symposium 2018, Maastricht (The Netherlands)

NVDO, Annual Dutch Diabetes Research Meeting 2018, Oosterbeek (The Netherlands)

European Atherosclerosis Society, Congress 2019, Maastricht (The Neterlands) 
European Association for the Studies of Diabetes, Annual Meeting 2018, Berlin (Germany)

\section{Poster presentations}

European Association for the Studies of Diabetes, Annual Meeting 2016, München (Germany)

European Association for the Studies of the Liver, International Liver Conference 2017, Amsterdam (The Netherlands)

European Fatty Liver Conference 2018, Vaalsbroek (The Netherlands)

European Association for the Studies of Diabetes, Annual Meeting 2020, Virtual

\section{Scientific awards}

Full Young Investigator Bursary, European Association for the Studies of the Liver, International Liver Conference 2017

One of the best six poster presentation, European Association for the Studies of the Liver, International Liver Conference 2017

Best poster presentation in the "Metabolic, alcoholic and toxicity" specialty

One of the best four abstracts, NVDO, Annual Dutch Diabetes Research Meeting 2017

Stimuleringsbeurs 2017 (t.w.v. $€$ 10.000), Erfelijke Stofwisselingsziekten Nederland

Second best poster presentation, European Fatty Liver Conference 2018 
